
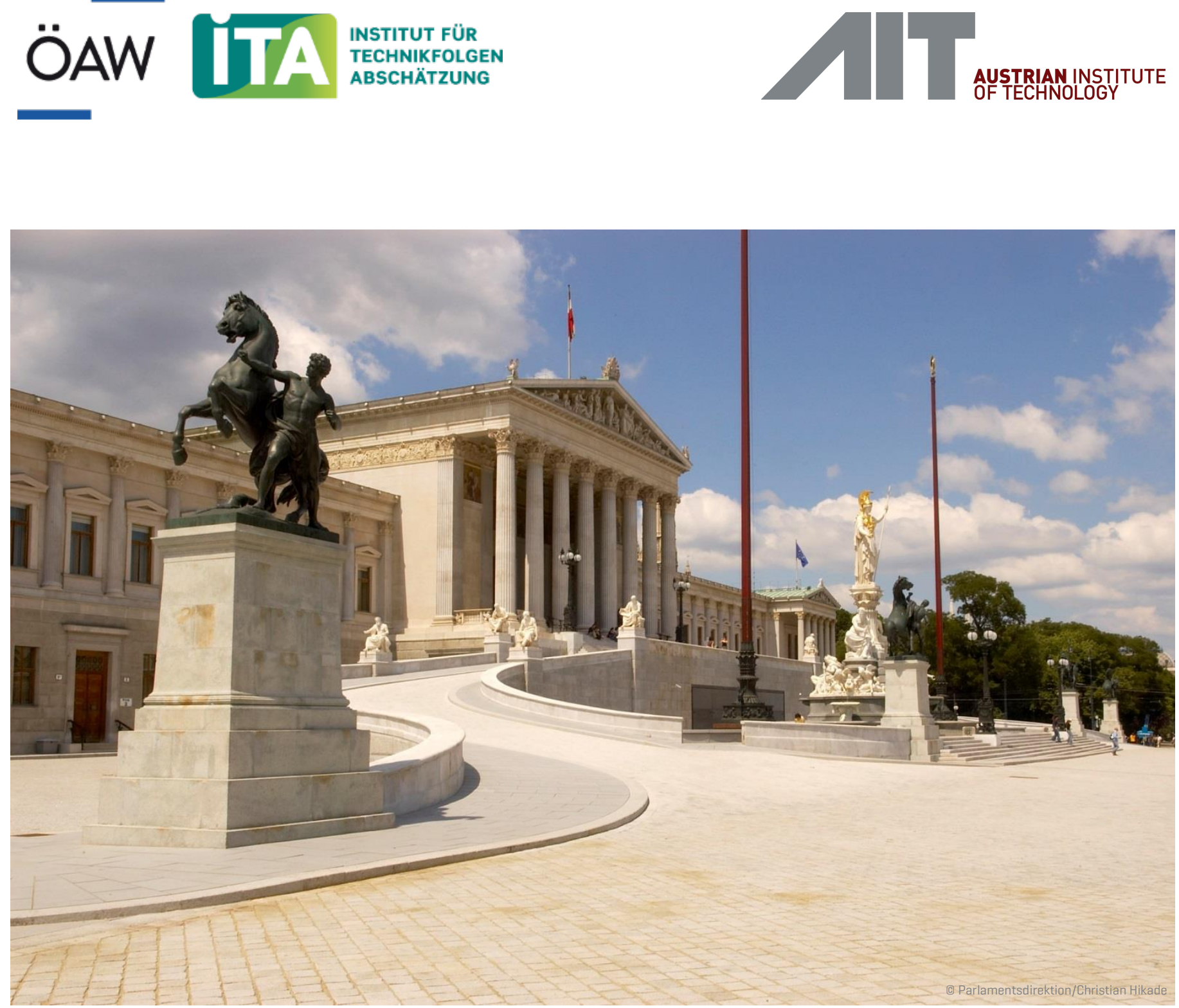

\title{
Foresight und
}

Technikfolgenabschätzung:

Monitoring von Zukunftsthemen für das Österreichische Parlament

Berichtsversion: November 2020 

Foresight und

Technikfolgenabschätzung:

Monitoring von Zukunftsthemen für das Österreichische Parlament

Berichtsversion: November 2020

Institut für Technikfolgen-Abschätzung [ITA]

der Österreichischen Akademie der Wissenschaften

AIT Austrian Institute of Technology

Center for Innovation Systems \& Policy

AutorInnen: Michael Nentwich [MN] [Projektleitung /ITA]

Matthias Weber [MW] [Projektleitung /AIT]

Dennis Appelt [DA]/ITA

Eva Buchinger [EB] /AIT

Leo Capari [LC] /ITA

Evgeniia Filippova [EF] /AIT

Niklas Gudowsky-Blatakes [NG] /ITA

Barbara Heller-Schuh [BHS] /AIT

Manuela Kienegger [MK] /AIT

Klaus Kubeczko [KK] /AIT

Michael Ornetzeder [MO] /ITA

Walter PeissI [WP] /ITA

Petra Schaper-Rinkel [PSR] /AIT

Anna Wang [AW] /AIT

Dana Wasserbacher [DW] /AIT

Studie im Auftrag des Österreichischen Parlaments

Wien, November 2020 


\section{IMPRESSUM}

Medieninhaber:

Österreichische Akademie der Wissenschaften

Juristische Person öffentlichen Rechts (BGBI 569/1921 idF BGBI I 31/2018)

Dr. Ignaz Seipel-Platz 2, A-1010 Wien

\section{Herausgeber:}

Institut für Technikfolgen-Abschätzung (ITA)

Apostelgasse 23, A-1030 Wien

AIT Austrian Institute of Technology

www.oeaw.ac.at/ita

Giefinggasse 4, A-1210 Wien

www.ait.ac.at

Die ITA- und AIT-Projektberichte erscheinen unregelmäßig und dienen der

Veröffentlichung von Forschungsergebnisse. Die ITA-Berichte werden über das

Internetportal „epub.oeaw“ der Öffentlichkeit zur Verfügung gestellt:

epub.oeaw.ac.at/ita/ita-projektberichte

Die AIT-Berichte werden über die Website ait.ac.at der Öffentlichkeit zur Verfügung gestellt:

ait.ac.at/ueber-das-ait/center/center-for-innovation-systems-policy/policy-advice-reports/

Projektbericht Nr.: ITA-AIT-13

ISSN: $\quad 1819-1320$

ISSN-online: 1818-6556

epub.oeaw.ac.at/ita/ita-projektberichte/ITA-AIT-13.pdf

parlament.gv.at/SERV/STUD/FTA/

(C) 2020 ITA-AIT - Alle Rechte vorbehalten 


\section{Inhalt}

1 Einleitung: Wozu und wie Monitoring? .

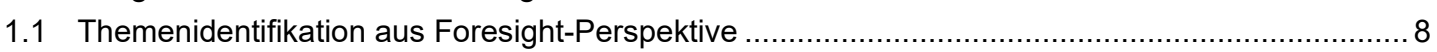

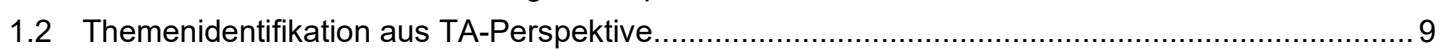

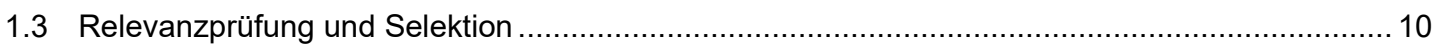

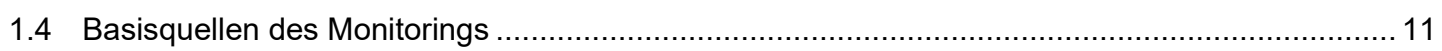

2 Für das Parlament und für Österreich relevante sozio-technische Entwicklungen ...................... 13

Parlament \& Demokratie

Zukunft des Internets: zentral vs. dezentral? [AKTUALISIERT]

Ein sicheres, dezentrales Grundbuch über Blockchain [AKTUALISIERT]

Einmal im Netz - immer im Netz? Technologien digitalen Vergessens

Digitales Nudging und Demokratie

Deepfakes - Perfekt gefälschte Bilder und Videos

Regulatorische Experimentierräume

Zukunft der Bewertungsplattformen: Online Reputationsmanagement

Robojournalismus und digitalisierte Medien

Microtargeting - Personalisierte Nachrichten zur Beeinflussung von Verhalten

Transparente Algorithmen - Wie lässt sich algorithmische Diskriminierung verhindern?

Staatliche Souveränität im digitalen Zeitalter

Digitalisierung und Anonymität - Ein Widerspruch in sich?

Arbeit, Gesundheit \& Soziales

Technische Arbeitsplatzüberwachung [NEU]

Pandemiemanagement mit technologischer Hilfe [NEU]

Exoskelette: Von digitalen Kampfanzügen zur futuristischen Gehhilfe [AKTUALISIERT]

Chips der Zukunft: Elektronische Haut [AKTUALISIERT]

Personalisierte Genomsequenzierung [AKTUALISIERT]

Automatisiertes Gesundheitsdaten-Monitoring [AKTUALISIERT]

Bionische Produktion der Zukunft: Selbstformende Objekte durch 4D-Druck [AKTUALISIERT]

Cyborg: Gehirn-Computer-Schnittstellen [AKTUALISIERT]

Künstliche Organe - 3D-Biodruck [AKTUALISIERT]

Funktionelle Nahrung aus dem Labor [AKTUALISIERT]

KI im Gesundheitswesen

Epigenetische Therapieansätze

Xenobots: lebendige Roboter?

Datengetriebene Medizin - Zwischen Personalisierung und gläsernen PatientInnen?

Zukunft der Mensch-Maschine-Interaktion: Haptische Holographie

Futuristische Sehhilfen

High-Tech-Nahrungsmittelsysteme

Der gen-editierte Mensch 
Bildung, Wissenschaft \& Kultur

„Right to Challenge" als alternatives Bewertungsverfahren für die Sharing Economy [NEU]

Künstliches Leben [AKTUALISIERT]

Digitale Erinnerung [AKTUALISIERT]

Social [Ro-]Bots: Maschinen als GefährtInnen? [AKTUALISIERT]

Quantenbiologie [AKTUALISIERT]

Digitales Lernen: Offene Infrastrukturen für Bildungsgerechtigkeit

Dezentrales KI-Lernen: Gesellschaft als Reallabor?

Open Access - jetzt aber wirklich?

Zukunft der Quantentechnologie

Biomimikry und Bionik: Designprinzipien aus der Natur

\section{Budget \& Finanzen}

Geldlose Tauschsysteme: Zeitbanken [AKTUALISIERT]

Sprunginnovationen: Neue Konzepte innovationsorientierter Industriepolitik

[AKTUALISIERT]

FinTechs - Revolution des Bankenwesens?

EU \& Außenpolitik

Europäische Resilienz in Krisenzeiten

Bergbau im All

Dezentralisierte Kollaborationsplattformen - Alternativen zu globalen Online-Monopolen

Inneres, Justiz \& Landesverteidigung

Sicherheits-Robotik [AKTUALISIERT]

Cybersicherheit für kritische Infrastrukturen [AKTUALISIERT]

Cybersicherheit: Vom Dialog zur Ko-Kreation [AKTUALISIERT]

Digitale Schutzengel: Technologien privater Sicherheit

Das Dark-Net

Algorithmische Polizeiarbeit

Fortgeschrittene Gesichtserkennung

Authentifizierung durch Verhalten

Existenzielle Risiken von Künstlicher Intelligenz

Umwelt, Infrastruktur \& Landwirtschaft

CO2 als Ressource [NEU]

Zukunft der Batterieentsorgung: Wohin mit ausgebrannten Elektroautos? [NEU]

Lichtverschmutzung [NEU]

Geräuschvolle Zukunft? [NEU]

Fernerkundung mit KI [NEU]

Mikroplastik - Abrieb der Zivilisation [AKTUALISIERT]

Vertrauenswürdige Blockchains [AKTUALISIERT]

Das Netz der bewegten Dinge [AKTUALISIERT]

Robotik in der Landwirtschaft [AKTUALISIERT]

Genome editing [CRISPR/Cas9] in der Pflanzenzucht [AKTUALISIERT] 
Treibstoffe aus Sonnenlicht: Künstliche Photosynthese und bionische Blätter [AKTUALISIERT]

Häuser aus dem 3D-Drucker [AKTUALISIERT]

Dienstleistung 4.0 [AKTUALISIERT]

Automatisierung in der Rechtsberatung [AKTUALISIERT]

Die Zukunft von Industrie 4.0 [AKTUALISIERT]

Von +Energie zu ++Energie: Zur Zukunft des Bauens

Peer-to-Peer[P2P]-Energiehandel

Pflanzen als vernetzte Umweltsensoren

$\mathrm{CO}_{2}$-neutrale Gebäudekühlung

Kommerzialisierung von Geoengineering-Technologien

5G - Gestaltungsoffenheit der Anwendungen für den neuen Mobilfunkstandard nutzen

Cloud Computing als politische Herausforderung

Autonomer öffentlicher Verkehr

Infrastruktur für Elektromobilität

Renaissance des Radverkehrs

Inwertsetzung von Natur

Illegaler Handel mit E-Schrott

Urban Mining 4.0

Lieferung auf der letzten Meile unter Nachhaltigkeitsgesichtspunkten

Zwischenspeicher der Zukunft für elektrische Energie

Grüner Wasserstoff 2.0

Plus Energie Quartiere: Zukunft der lokalen Energieversorgung?

Smarte Straßen

Offene Mobilitätsplattformen zur Unterstützung der Verkehrswende?

Zukunft Lieferdrohnen?

\section{Wirtschaft \& Innovation}

Point of Sale 2.0: Automaten der Zukunft [NEU]

Personalisierte Ernährung: Nudges statt Wedges? [NEU]

Virtuelle und augmentierte Realitäten [AKTUALISIERT]

Sensorrevolution: Smarte Städte - smarte Menschen? [AKTUALISIERT]

Fliegende Windenergie [AKTUALISIERT]

Wasserstoffspeicher der Zukunft [AKTUALISIERT]

Autonome Mini-Häuser [AKTUALISIERT]

Zukunft der Mensch-Maschine-Interaktion: Spracherkennung und -steuerung

[AKTUALISIERT]

Roboterautos [AKTUALISIERT]

Selbstheilende Materialien [AKTUALISIERT]

Industrie 4.0 und Bioökonomie

Smart Spaces

Lebensmitteltracking

Metalinsen 
Affective Computing - Emotionale Künstliche Intelligenz

Gamification von Wissenschaft, Arbeit und Politik?

Intelligente und funktionelle Oberflächen für industrielle Anwendungen der Zukunft

Frugale Innovation für heiße Sommer

Biobasierte Zukunftsmaterialien: Vom Laborleder bis zum Superholz

Zellfabriken der Zukunft 


\section{Einleitung: Wozu und wie Monitoring?}

Ein kontinuierliches Monitoring aktueller oder sich für die Zukunft abzeichnender internationaler wissenschaftlicher und technologischer Entwicklungen im gesellschaftlichen Kontext (sozio-technische Trends) ist die Grundlage, um zentrale Zukunftsthemen für die österreichische Politik zu identifizieren. In so einem Verfahren werden zudem wichtige wissenschaftlichtechnische Treiber für Veränderungen sichtbar (drivers of change), die dem Parlament bei frühzeitiger Berücksichtigung erweiterte Handlungsund Gestaltungsmöglichkeiten eröffnen. Ein Monitoring ist damit zugleich die Grundlage für vertiefende Studien im Bereich Foresight und Technikfolgenabschätzung (TA). Somit wird es möglich, später aufkommende, spezifische und tagesaktuell drängende Fragen in breiteren Zukunftsthemen zu verorten und die jeweilige Relevanz schneller und vorausschauend zu beurteilen. Die Ergebnisse des Monitorings unterstützen damit nicht nur eine vorausschauende FTI-Politik, sondern dienen mit ihrer TA-Komponente auch der Maximierung positiver und zugleich Minimierung möglicher negativer Technikfolgen und sind damit auch für andere Politikfelder hochrelevant. Die potentiellen Anwendungsfelder von Zukunftstechnologien sind mit hohen Erwartungen und vielfältigen Versprechen verbunden. Während der Umsetzung zeigt sich aber oft, dass mit diesen Erwartungen und Versprechen auch Effekte einhergehen, die zunächst nicht augenscheinlich sind. Die Foresight-Komponente setzt auf die Gestaltbarkeit von Innovationen: Werden die Potentiale von Zukunftstechnologien frühzeitig in ihrer Bandbreite analysiert, eröffnen sich Gestaltungsspielräume für nachhaltige Innovationspfade.

Das zeigt, dass eine verantwortungsvolle und zukunftsorientierte Technikentwicklung insbesondere den Fokus auf zwei Dimensionen legen sollte, die beide mit Foresight und TA bearbeitbar sind:

- zum einen auf den Handlungsspielraum und die Bedingungen, unter denen aus wissenschaftlich-technischen Potentialen tatsächlich wirtschaftlich und gesellschaftlich relevante Innovationen werden;

- zum anderen auf die möglichen Folgen sozio-technischer Entwicklungen in Hinblick auf Gesundheit, Umwelt, Wirtschaft, Recht und Gesellschaft.

Dieser Abschnitt beschreibt einleitend, wie das Monitoring durchgeführt wurde. Die beiden Partner, ITA und AIT, ergänzen sich in Hinblick auf die Identifikation von relevanten Themen und schöpfen dadurch Synergieeffekte aus: Während das AIT auf reichhaltige Erfahrung im ForesightBereich zurückgreift, bezieht sich das ITA auf die in der Technikfolgenabschätzung übliche Vorgangsweise.

Der Foresight-Ansatz des AIT identifiziert relevante Technologien aufgrund ihrer Potentiale zur Lösung gesellschaftlicher Herausforderungen. Das ITA orientiert sich an einem problemorientierten Ansatz. Hierbei stehen vor allem technologieinduzierte, potentiell problematische Effekte im Vordergrund, die durch die Implementierung entstehen können.
Identifikation zentraler

Zukunftsthemen für die österreichische Politik

Unterstützung der FTI-Politik und Umgang mit Technikfolgen

zwei Dimensionen verantwortungsvoller und zukunftsorientierter Technikentwicklung

Kombination von ...

... Foresight
und Technikfolgen-
abschätzung


Zeithorizont:

5-10 Jahre
Foresight hat im Hinblick auf sozio-technische Trends in der Regel einen längeren zeitlichen Horizont ( $a b$ zehn Jahren) im Blick, wohingegen TA einen kürzeren zeitlichen Horizont aufweist (bis fünf Jahre). Durch die Kombination dieser Ansätze (gestaltungsorientiert, problemorientiert, langbzw. kurzfristig) können Technologien identifiziert werden, die kurz- und mittelfristig Handlungsbedarf nach sich ziehen.

\subsection{Themenidentifikation aus Foresight-Perspektive}

gesellschaftliche Herausforderungen im Fokus

neue wissenschaftlichtechnische

Entwicklungen und ihre möglichen Anwendungsfelder
Um den gesellschaftlichen Herausforderungen der Zukunft gerecht zu werden, bedürfen die Identifikation und die Bewertung von potentiell relevanten Technologien und Anwendungen eines Rahmens, der außerhalb der technologischen Entwicklungen liegt. Zusätzlich zu den etablierten Maßstäben von wirtschaftlichem Wachstum und internationaler Wettbewerbsfähigkeit werden gesellschaftliche Herausforderungen berücksichtigt: die Bedeutung von Zukunftstechnologien für Herausforderungen für die Bearbeitung von Klimawandel, Energieversorgung und demografischen Wandel ${ }^{1}$ oder auch - sehr aktuell - die Bedeutung dieser Technologien zur Bearbeitung der international vereinbarten Nachhaltigkeitsziele (SDGs). ${ }^{2}$

Für die folgenden Themen wurden aktuelle technologische Entwicklungen und aktuelle Herausforderungen in eine Matrix zusammengefügt, die einerseits Technologien und andererseits Themenfelder aktueller gesellschaftlicher Herausforderungen abbildet. Um die technologischen Entwicklungen adäquat strukturieren und klassifizieren zu können, verwenden wir die OECD-Systematik der Felder von Wissenschaft und Technologie. ${ }^{3}$ Diese ermöglichen es, neue wissenschaftlich-technische Entwicklungen entsprechend zu kontextualisieren. Bei neu aufkommenden Technologien kommt es dabei zu Mehrfachzuordnungen, da neue Technologien sowohl in der Forschung selbst eine hohe Anwendung haben, als auch in angewandten Bereichen (z. B. Gene Editing/CRISPR/Cas9 in Biologie, in der Umweltbiotechnologie, in den Gesundheitswissenschaften).

Neue wissenschaftlich-technische Entwicklungen werden damit in Relation zu möglichen Anwendungsfeldern gesetzt. Als Heuristik zur Strukturierung relevanter Felder wurden die globalen Nachhaltigkeitsziele (SDGs) gewählt, da sie umfassender und genauer als die üblichen großen gesellschaftlichen Herausforderungen wirtschaftliche und gesellschaftliche Be-

1 So bot die Lund Deklaration (2009, Europe must Focus on the Grand Challenges of our Time, Swedish EU Presidency) die Grundlage für die Challenge-Orientierung des Europäischen Forschungsrahmenprogramms Horizon 2020.

2 United Nations (2015) Transforming our world: The 2030 agenda for sustainable development, New York: United Nations, Department of Economic and Social Affairs, un.org/Depts/german/gv-70/band1/ar70001.pdf (zuletzt aufgerufen am 21.05.2018. Alle weiteren in diesem Bericht zitierten URLs wurden zuletzt zum Datum der jeweiligen letzten Aktualisierung, siehe Fußzeile, überprüft).

3 Die Fields of Science and Technology (FOS) ist eine von der OECD festgesetzte Systematik von Wissenschaftszweigen. 
darfe repräsentieren. Damit wird sichtbar, welche Technologien eine potentiell hohe Bedeutung für unterschiedliche Ziele wie nachhaltiges Wirtschaftswachstum, nachhaltige Konsum- und Produktionsmuster, Schutz von Ökosystemen, inklusive Institutionen, Ernährungssicherheit, Gesundheit, Bildung, Energie etc. haben.

\subsection{Themenidentifikation aus TA-Perspektive}

Aus Perspektive der Technikfolgenabschätzung erscheint es besonders relevant, jene Themen zu identifizieren, die kurz- bis mittelfristig politischen Handlungsbedarf nach sich ziehen könnten. Das betrifft insbesondere sozio-technische Entwicklungen, die möglicherweise problematische Auswirkungen auf Gesundheit, Umwelt, Wirtschaft, Recht oder Gesellschaft haben könnten, aber auch solche, deren Förderung zu frühzeitigen, positiven gesellschaftlichen Effekten führen kann.

Um solche Themen zu finden, führte das ITA-Team eine komprimierte Variante seines laufenden [meTAscan]-Verfahrens durch. Dabei handelt es sich um eine informierte Auswahl aus spezifischen Sekundärquellen, die wichtige zukünftige Entwicklungen beschreiben (siehe Abschnitt 1.4). Im ersten Schritt wird eine Primärdatenbank sozio-technischer Entwicklungen erstellt. Bei dieser Quellenauswertung handelt es sich um einen laufenden und dynamischen Prozess, d. h. es wird in regelmäßigen Abständen nach neuen Quellen recherchiert, die dann in die Primärdatenbank der soziotechnischen Entwicklungen eingepflegt werden. Dies ist notwendig, um mit der hohen Dynamik der Technologieentwicklung mithalten zu können.

Auf diese Weise werden laufend aktuelle sozio-technische Entwicklungen gefunden und anschließend in einem Bottom-up-Prozess Clustern zugeordnet. ${ }^{4}$ Danach wurden jene Entwicklungen ausgeschieden, die aus ExpertInnen-Sicht bereits ausreichend abgehandelt sind, eher Science-Fiction-Charakter haben bzw. auf den ersten Blick für Österreich irrelevant scheinen.

Im nächsten Schritt wurden alle Einträge der aktualisierten und geclusterten Primärdatenbank sozio-technischer Entwicklungen parallel durch die beteiligten TA-ExpertInnen entsprechend den Kriterien für Relevanz aus TA-Perspektive eingeschätzt. Diese EHS ${ }^{5}$ - und ELSI ${ }^{6}-$ Kriterien können in folgenden Fragen beschrieben werden:

4 Ursprünglich: Bergbau; Big Data; Bildung; Computertechnologie; Crowdsourcing; Digitale Wirtschaft; Energie; Genomics; Gesundheitstechnologien; Industrielle Produktion; Informations- und Kommunikationstechnologien; Internet der Dinge; Klimatechnologie; Künstliche Intelligenz; Landwirtschaft; Mensch-Maschine-Schnittstellen-Technologie; Messen und Visualisierung; Mobilität; Nachahmung der Natur und Cyborgs; Neue Arbeit; Neue Werkstoffe; Neurotechnologien; Robotik; Synthetische Biologie; Überwachung.

5 EHS steht für „Environmental, Health and Safety“, also Umwelt-, Gesundheits- und Sicherheitsaspekte. kurz- bis mittelfristiger politischer Handlungsbedarf im Fokus

\section{Auswertung von} Studien zu zukünftigen sozio-technischen Entwicklungen

Relevanzprüfung nach TA-Kriterien: EHS \& ELSI 
Gibt es Hinweise auf mögliche

i. Gesundheits- oder Umweltwirkungen;

ii. ethische Implikationen;

iii. bevorstehende politische oder schleichende gesellschaftliche Debatten; oder

iv. gesellschaftliche oder kulturelle Auswirkungen?

\subsection{Relevanzprüfung und Selektion}

Auswahl durch Gruppe von TA- und ForesightExpertInnen ...

... anhand folgender Fragen
Die kritische Reflexion der gefundenen sozio-technischen Entwicklungen unter Zuhilfenahme der Fragen i-iv ermöglicht die Identifikation wesentlicher Relevanzaspekte. Die wichtigsten Aspekte wurden dokumentiert, wobei auch die Österreich- und Parlamentsrelevanz angesprochen wurden. Unterschiedliche Einschätzungen durch die beteiligten ExpertInnen wurden ausdiskutiert. Jene Entwicklungen, die übereinstimmend von den beteiligten TA- und Foresight-ExpertInnen als potenziell relevante und drängende Themen eingestuft wurden, bildeten das Zwischenergebnis.

In einem gemeinsamen Workshop erfolgte im nächsten Schritt die Zusammenführung der aus den beiden Perspektiven als wichtig erkannten sozio-technischen Entwicklungen. In der folgenden ExpertInnen-Diskussion erfolgte eine Prüfung und Reihung der Entwicklungen auf parlamentarische und auf Österreich-Relevanz. Hier wurden einerseits Potentiale identifiziert, die einen Beitrag zur Bewältigung der Grand Challenges bzw. zur Erreichung der UN-Ziele einer nachhaltigen Entwicklung beitragen können, und andererseits überprüft, wie eng der Bezug zu Österreich/zum Parlament sein kann. Es wurden folgende Fragen für potentielle Themen diskursiv beantwortet:

- Besteht hier ein Innovationspotential in Österreich, welches über geeignete Maßnahmen ausgeschöpft werden kann?

- Sind gewisse Bereiche der sozio-technischen Entwicklung abzusehen in denen in nächster Zeit politische Handlungen gesetzt werden könnten/ sollten?

- Passen bestimmte Entwicklungen in soeben anstehende Agenden der parlamentarischen Ausschüsse aufgrund von Themenübereinstimmung?

Das Ergebnis dieses Prozesses, d. h. die Auswahl von insgesamt 100 derzeit besonders relevanten und aktuellen sozio-technischen Entwicklungen ist in Kapitel 2 dokumentiert. Dabei werden die oben gestellten Fragen pro Thema überblicksartig beantwortet.

6 ELSI steht für „Ethical, Legal and Societal Implicatons“, also ethische, rechtliche und gesellschaftliche Wirkungen. 


\subsection{Basisquellen des Monitorings}

Als Quellen dienten für diesen Bericht folgende Sekundärquellen und Datenbanken in der angeführten bzw. in der jeweils aktuellsten Version:

- 100 Opportunities for Finland and the World (2014)

- 100 Radical Innovation Breakthroughs for the future (2019)

- AIT - Foresight-Datenbank Studien

- Cranfield Futures (Horizon scans) ${ }^{7}$

- European Strategy and Policy Analysis System (ESPAS-Datenbank) ${ }^{8}$

- Foresight Functional Materials Taskforce - Functional Materials Future Directions

- Forschungs- und Technologieperspektiven 2030 - Ergebnisband 2 zur Suchphase von BMBF-Foresight Zyklus II

- Gartner Top 10 Strategic Technology Trends (ab 2017)

- Global Change Blog (Futurist Blog)

- Global Trendometer, European Parliamentary Research Service $(a b 2018)^{9}$

- Governmental Accountability Office (GAO) - Data and Analytics Innovation

- Dossiers \& Berichte des Instituts für Technikfolgen-Abschätzung (ITA)

- Key Enabling Technologies (KETs) Observatory

- Metascan 3 - Emerging Technologies

- Millenium Project: State of the Future Report

- OBSERVE Horizon Scanning Report, Fraunhofer ISI (2016) ${ }^{10}$

- OECD Science, Technology and Innovation Outlook

- RIBRI Consultation ${ }^{11}$

- Society and Lifestyles in 2050 (Institute for Global Environmental Strategies 2019)

- Studien und "Notes" des Parliamentary Office of Science and Technology (POST)

- Studien und Publikationen des Europäischen Parlaments/Science and Technology Options Assessment

- Studien und Publikationen des TAB - Büro für TechnikfolgenAbschätzung beim Deutschen Bundestag

7 web.archive.org/web/20160914115240/http:/www.cranfieldfutures.com/horizonscanning-database/.

8 espas.secure.europarl.europa.eu/orbis/.

9 europarl.europa.eu/thinktank/en/document.html?reference=EPRS_STU[2018]612835; europarl.europa.eu/RegData/etudes/STUD/2019/646111/EPRS_STU[2019]646111_ EN.pdf.

10 horizon-observatory.eu/radar-en/downloads/deliverables.php.

11 ribri-consultation.eu. 
- Teknologiradet Policy Briefs

- TIM-Tools for Innovation Monitoring ${ }^{12}$

- U.S. Department of Health and Human Services: 2020 A New Vision - A Future for Regenerative Medicine

- Weak signals in Science and Technologies: 2019 Report

- World Economic Forum, The Global Risks Report

- World Economic Forum-Top 10 Emerging Technologies (ab 2016)

- World Technology Evaluation Center - Report:

Applications: Nanodevices, Nanoelectronics, and Nanosensors sowie allgemein:

- Klassische wissenschaftliche Publikationen

- Journalistische Medien und Internetquellen

12 timanalytics.eu. 


\section{Für das Parlament und für Österreich relevante sozio-technische Entwicklungen}

Die folgenden sozio-technischen Entwicklungen wurden als besonders relevante und aktuelle Themen für das Parlament und für Österreich identifiziert. Die Auswahl zeigt ein breites Spektrum an Themen mit weitreichenden sozialen, ökonomischen, politischen und ökologischen Auswirkungen. In diesem Bericht wurden sie den von der Parlamentsdirektion vorgeschlagenen, auch innerhalb des Parlaments in verschiedenen Kontexten verwendeten acht Clustern zugeordnet, die grob die Ausschussstruktur widerspiegeln:

- Parlament \& Demokratie

- Arbeit, Gesundheit \& Soziales

- Bildung, Wissenschaft \& Kultur

- Budget \& Finanzen
- EU \& Außenpolitik

- Inneres, Justiz \& Landesverteidigung

- Umwelt, Infrastruktur \& Landwirtschaft

- Wirtschaft \& Innovation

In all diesen Bereichen hat Österreich Kompetenzen vorzuweisen, die aus Sicht der Forschungs-, Innovations- und Technologiepolitik wirtschaftliche Entwicklungspotentiale darstellen. Zugleich zeigen diese sozio-technischen Entwicklungen neuen parlamentarischen Handlungsbedarf als auch parlamentarische Gestaltungsspielräume - jeweils in einem breiteren gesamtgesellschaftlichen Kontext (z. B. KonsumentInnenschutz).

Im Folgenden werden die 110 identifizierten sozio-technische Entwicklungen vorgestellt. Von diesen sind 28 vertieft dargestellt und durch Vorschläge für die weitere parlamentarische Bearbeitung ergänzt. Zehn Themen wurden in diesem Bericht neu aufgenommen, davon wiederrum vier vertieft. Zusätzlich wurden 37 Themen aus früheren Berichten aktualisiert, da seit der Erstbearbeitung wesentliche Neuentwicklungen stattgefunden haben. Das sind die in diesem Bericht neu hinzugekommenen bzw. aktualisierten sozio-technischen Entwicklungen:

\begin{tabular}{llll}
\hline Neue Themen & Aktualisierte Themen & & \\
\hline Arbeitsplatzüberwachung & Künstliches Leben & Genome Editing für Pflanzen & Automatisierte Rechtsberatung \\
Bewertung Sharing-Economy & Blockchain-Services & 3D-Biodruck & Blockchain-Grundbuch \\
Lichtverschmutzung & Funktionelle Nahrung & Künstliche Photosynthese & Industrie 4.0 \\
Lärmminderung & Digitale Erinnerung & Wasserstoff-Speicherung & Gesundheitsdatenmonitoring \\
Pandemiemanagement & Sicherheits-Robotik & Elektronische Haut & Sprachassistenten \\
KI-Umweltfernerkundung & Netz der bewegten Dinge & Cyborg & Roboterautos \\
E-Mobilität: & Cybersicherheit: & Häuser aus dem & Cybersecurity: \\
Batterieentsorgung & Infrastruktur & 3D-Drucker & Ko-Kreation \\
$\mathrm{CO}_{2}$ als Ressource & Social [Ro-]Bots & Autonome Minihäuser & Dezentrales Internet \\
Personalisierte Ernährung & Sensorrevolution & Zeitbanken & Sprunginnovationen fördern \\
Automatenindustrie & Agrar-Robotik & 4D-Druck & Selbstheilende Materialien \\
& Fliegende Windenergie & Quantenbiologie & Mikroplastik \\
& Personalisierte & Dienstleistung 4.0 & Exoskelette \\
& Genomsequenzierung & Virtuelle Realitäten \\
\hline
\end{tabular}


In der folgenden Graphik werden alle 110 Themen gemeinsam dargestellt und den oben genannten Clustern zugeordnet sowie deren thematische Verknüpfungen auch zu anderen Clustern (sprich: politischen Themenfeldern) dargestellt. Die in dieser Berichtsversion neu hinzugekommenen sozio-technischen Entwicklungen sind fett dargestellt.

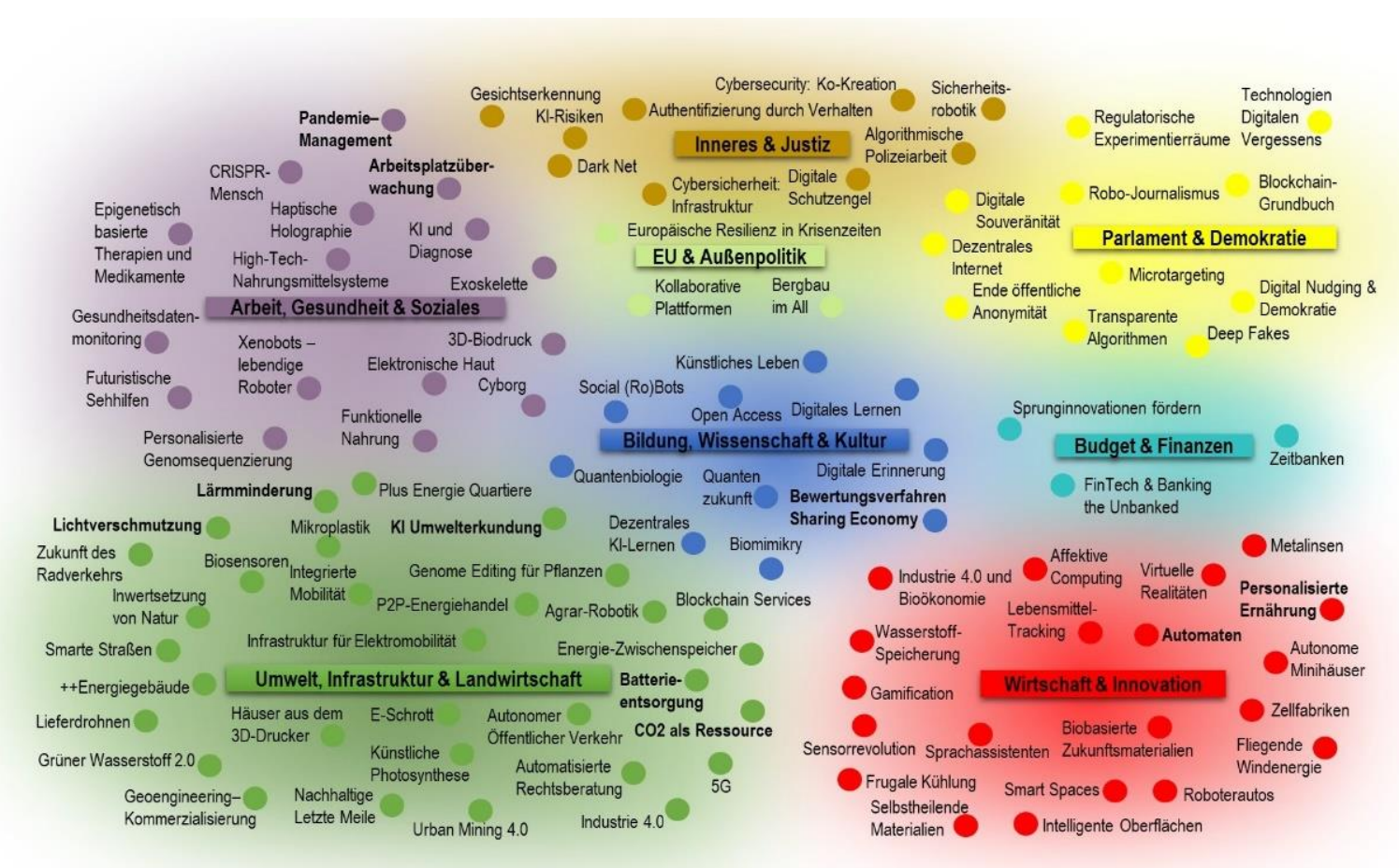

Abbildung: 110 sozio-technische Entwicklungen mit Relevanz für Österreich und das Parlament 


\section{Zukunft des Internets: zentral vs. dezentral?}

Das Internet basiert auf zwei Komponenten: der technischen Infrastruktur (WLAN/UMTS, Kupfer- oder Glasfaserkabel bzw. DSL) und der Dateninfrastruktur, die in den meisten Ländern mehrheitlich von privaten Anbietern bereitgestellt wird (Jetzke/Richter 2016). Das neunte nachhaltige Entwicklungsziel der Vereinten Nationen fordert bis 2020 vermehrte Bemühungen, umfassenden und leistbaren Internetzugang bereitzustellen, vor allem in den am wenigsten entwickelten Ländern (United Nations 2015). Ursprünglich vom Erfinder des World Wide Web, Tim Berners-Lee, als dezentrales Netzwerk designt, unterliegt es auf beiden Ebenen seiner Infrastruktur (technisch und informationell) in den letzten Jahren erheblichen Zentralisierungstendenzen. ${ }^{1}$

Das verdeutlichen neue Geschäftsmodelle globaler Internetkonzerne: Google und Facebook forschen an neuen Mobilfunktechnologien, vor allem an kostengünstigen drahtlosen Übertragungswegen, wie Drohnen, Ballons und Satelliten (Jetzke/Richter 2016). Die Innovationsbestrebungen der Unternehmen treiben einerseits die Entwicklung dezentraler technischer Infrastruktur voran, bergen andererseits auch Risiken in Hinblick auf die Dateninfrastruktur: Als Netzbetreiber können sie durch das sogenannte „Zero-Rating“ bestimmte Online-Angebote kostenlos zur Verfügung stellen und die Nutzung der ausgewählten Dienste damit bevorzugen (da die Errichtung neuer Infrastruktur eine hohe Markteintrittsbarriere für neue Marktteilnehmer darstellt). Unter dem Schlagwort "Netzkolonialismus“ kritisieren Aktivistlnnen der Netzneutralität das Geschäftsmodell, das vor allem zur Gewinnung von Marktanteilen in Entwicklungs- und Schwellenländern eingesetzt wird. Das Beispiel Wikipedia Zero in Angola zeigt, dass Nutzungsgebote, die durch gratis Zugangsmöglichkeiten forciert werden, auch in die andere Richtung ausschlagen können: Mit dem Mobilfunkbetreiber wurde ein kostenloser Zugang zu Wikipedia-Angeboten ausgehandelt; in Folge wurde das zur Verfügung gestellte Datenvolumen zum illegalen Austausch von Filmen und Videospielen genutzt, oft als Bild- oder Textdatei getarnt. ${ }^{2}$

Was sind in Anbetracht der zunehmenden digitalen Macht einiger weniger, Möglichkeiten, den Zugang zum Internet zu demokratisieren und die Datenhoheit zu dezentralisieren? Eine Option ist es, die physisch-technischen Datenspeicher von Datenservices zu entkoppeln und damit die vorab getroffenen Entscheidungen von Internetanbietern aufzuheben, um freie, eigenständige Entscheidungen im Zusammenhang mit den eigenen Daten treffen zu können. Beispiele für die Implementierung sogenannter selbstbestimmter „Personal Data Pods“ sind das Solid Projekt ${ }^{3}$ von Tim

\footnotetext{
1 ruben.verborgh.org/articles/redecentralizing-the-web/.

2 sueddeutsche.de/digital/nachrichten-aus-dem-netz-vielen-dank-fuer-dasdatenvolumen-1.2932453.

3 solidproject.org.
} 
Berners-Lee oder Mastodon ${ }^{4}$, ein dezentraler Twitter-ähnlicher Service von Eugen Rochko. Mit diesen Services kann die Entscheidung, auf welchen Servern persönliche Daten gespeichert werden, vom User selbst getroffen werden. Was einerseits die Selbstbestimmung von Usern erhöht, kann andererseits die Grenzen der Filterblasen weiter verhärten: der Zustrom zu Mastodon wurde auch von der Möglichkeit, pornografische Inhalte zu teilen, angetrieben. ${ }^{5}$ Andererseits zeichnet sich gegenwärtig ein Trend $a b$, der deutlich macht, dass das Interesse an technischer Internetinfrastruktur ständig wächst: Neben überregionalen Handynetzbetreibern ersteigerten in den ersten Auktionen in Österreich auch kommunale Unternehmen, wie z. B. die Graz Holding AG, Frequenzen für 5G-Mobilfunk, um regionale Netze aufzubauen (siehe Thema $5 G$ - Gestaltungsoffenheit der Anwendungen für den neuen Mobilfunkstandard nutzen).

Wie sich die Eigentumsverhältnisse in der Internetinfrastruktur weiterentwickeln und wie sich die Datenarchitekturen von Servicediensten zukünftig verändern, wird Parameter wie die Netzneutralität ${ }^{6}$ beeinflussen. Um in Zukunft einen fairen und leistbaren Internetzugang für alle Menschen garantieren und damit zusammenhängende regulative Herausforderungen meistern zu können, empfiehlt sich eine kontinuierliche Beobachtung von Infrastrukturtrends und Nutzungsdynamiken im globalen Internet.

\section{Zitierte Literatur}

Bullinger, G. M., 2010, Sachstand: Netzneutralität. Pro und Contra einer gesetzlichen Festschreibung, Berlin: Deutscher Bundestag.

Jetzke, T. und Richter, S., 2016, Netzkolonialismus, in: TAB, Themenkurzprofil No. 6, Berlin: Büro für Technikfolgen-Abschätzung beim deutschen Bundestag.

United Nations, 2015, Transforming our world: The 2030 agenda for sustainable development, New York: United Nations, Department of Economic and Social Affairs.

\footnotetext{
4 mastodon.social/about.
}

5 ethanzuckerman.com/blog/2017/08/18/mastodon-is-big-in-japan-the-reasonwhy-is-uncomfortable/.

6 Netzneutralität meint vordergründig die Gleichbehandlung von Daten bei der Übertragung im Internet, ist aber ein vieldiskutierter und uneindeutiger Begriff. Zur Diskussion um die gesetzliche Festschreibung der Netzneutralität in Deutschland siehe Bullinger (2010). 


\section{Ein sicheres, dezentrales Grundbuch über Blockchain}

Das Grundbuch als ein von den Bezirksgerichten geführtes öffentliches Verzeichnis, in dem Grundstücke und die an ihnen bestehenden dinglichen Rechte dokumentiert sind, war eine der Anwendungen, die als Favoriten galten, um in eine Blockchain überführt zu werden. Einige Staaten (z. B. Honduras ${ }^{1}$, Ukraine, Schweden) haben begonnen, digitale Grundbücher mit Blockchain einzuführen oder prüfen die Möglichkeit einer solchen Einführung. Ein Blockchain-Grundbuch wurde als sicher vor Manipulation angesehen, als stark kostensenkend und als Mittel, Korruption einen Riegel vorzuschieben. Mittlerweile zeigt sich, dass die Schwierigkeiten der Realisierung größer sind, als vor wenigen Jahren angenommen und dass die Einführung verteilter Datenbanken in dem Sektor zwar stattfindet, dass die Einführung aber im Vergleich zu anderen Sektoren langsamer verläuft als erwartet (Bennett, Pickering, \& Sargent, 2019). Eine Arbeit spielt die Ei-führung der Blockchain im deutschen Grundbuchwesen durch und zeigt hier Fallstricke und Lösungen auf (Simmchen, 2020).

Allerdings sind viele Ansätze primär über private Firmen realisiert worden und es gibt keine Langfristerfahrungen. Offene Fragen sind, wie sich die Transitionsphase von zentralen Systemen zu dem dezentralen System organisieren lässt und ob das System langfristig tatsächlicher günstiger ist. Eine Implementierung von Blockchain Anwendungen im öffentlichen Sektor bedarf der Klärung zentraler Voraussetzungen. Die Technologie kann nur für staatliche Anwendungen eingesetzt werden, wenn es eine offiziell anerkannte, digitale Identität gibt und schließlich wäre ein umfassender KnowHow-Aufbau und Schulungsaufwand innerhalb der Verwaltung erforderlich, um Blockchain-Projekte umsetzen zu können.

Grundbücher haben sich zur Gestaltung konkreter Prototypen geeignet, da sie hochgradig formalisiert sind. Die bisherigen Erfahrungen in einigen Ländern verweisen aber nicht darauf, dass es eine hohe globale Dynamik in der Einführung geben wird (Bennett et al., 2019).

\section{Zitierte Literatur}

Bennett, R. M., Pickering, M., \& Sargent, J. (2019). Transformations, transitions, or tall tales? A global review of the uptake and impact of NoSQL, blockchain, and big data analytics on the land administration sector. Land Use Policy, 83, 435-448. doi.org/10.1016/j.landusepol.2019.02.016.

Lemieux, V. L. (2016). Trusting records: is Blockchain technology the answer? Records Management Journal.

\footnotetext{
1 Das System in Honduras beruht allerdings auf der Bitcoin-Blockchain und wird von einem privaten Unternehmen implementiert. Damit sind vielfältige Sicherheitsrisiken verbunden, da es keine staatliche Kontrolle über die Infrastruktur gibt, siehe: Lemieux (2016).
} 
Lemieux, V. L. (2016). Trusting records: is Blockchain technology the answer? Records Management Journal, 26(2), 110-139. doi:10.1108/rmj-12-2015-0042.

Simmchen, C. (2020). Grundbuch ex machina: Eine kritische Untersuchung zum Einsatz der Blockchain im Grundbuchwesen (1 ed. Vol. 42). Baden-Baden: Nomos Verlagsgesellschaft mbH \& Co. KG. 


\section{Einmal im Netz - immer im Netz? Technologien digitalen Vergessens}

Wie wird in Zukunft „digitales Vergessen“ möglich sein? Digitales Vergessen umfasst so unterschiedliches wie die Löschung von Daten aus dem quasi digitalen Kurzzeitgedächtnis, ${ }^{1}$ die Forderung nach Löschung von Daten in einer Weise, dass sie nicht "für immer" im digitalen Langzeitgedächtnis verbleiben („digitaler Radiergummi“) ${ }^{2}$ und schließlich Ansätze, die vorwiegend auf die Information von NutzerInnen abzielen. Bisher gibt es schwer durchsetzbare Rechtsansprüche auf Löschung und die Datenschutzprinzipien der Datensparsamkeit bzw. Datenvermeidung und der informationellen Selbstbestimmung, aber keine Konzepte, die das Problem längerfristig und ganzheitlich angehen.

Seit dem Skandal rund um Facebook und Cambridge Analytica ${ }^{3}$ wissen wir, dass Technologieunternehmen oft mehr Daten speichern, als sie vorgeben und es für einzelne Nutzerlnnen immer schwieriger wird, den Überblick über die eigenen, personenbezogenen Daten im Netz zu behalten. Mit zunehmenden Datenmengen steigen auch die Möglichkeiten des Datenmissbrauchs, z.B. in Form von Hacker-Angriffen, die in unterschiedlicher Weise danach trachten, einzelne, persönliche Identitätsmerkmale zu stehlen. In Anbetracht fehlender Nutzungsalternativen und mangelndem Bewusstsein für den eigenen digitalen Fußabdruck, vor allem bei jungen Menschen (Vervier et al. 2017), stellt sich die Frage, wie zukünftig die Integrität von Online-Persönlichkeiten gesichert werden kann. Zur Integrität zählt ganz zentral die Kontrolle über individuelle Daten und damit auch über die Daten, die aus den unterschiedlichsten Gründen gerade nicht öffentlich verfügbar sein sollten, und somit auch das Recht auf „digitales Vergessen".4

Technologien digitalen Vergessens sind unterschiedlich konzeptualisiert: Zum einen sind es Technologien, die darauf abzielen zu verhindern, dass überhaupt personenbezogene Daten gesammelt werden können. Das sind z.B. Maßnahmen wie das Blockieren von Trackingscripts, das automatische Wechseln von User Agents, das automatische Verbinden über HTTPS, das Deaktivieren von JavaScripts, das Nicht-Aufzeichnen des Browserverlaufs u.v.m. Es gibt bereits Apps, die diese Technologien ver-

1 In Österreich kann die Löschung von Daten gemäß Artikel 17 der DatenschutzGrundverordnung mit Hilfe eines Formblatts der Datenschutzbehörde beantragt werden.

2 zeit.de/digital/datenschutz/2011-01/radiergummi-vergessenschoenberger/komplettansicht.

3 derstandard.at/2000076457187/Cambridge-Analytica-Datenskandalerschuettert-Facebook.

4 Das Konzept des digitalen Vergessens ist durch den österreichischen Wissenschaftler Mayer-Schönberger (2009) bekannt geworden. 
einen und dadurch höchstmöglichen Datenschutz versprechen. ${ }^{5}$ Zum anderen sind es Technologien, die das Internet auf personenbezogene Daten durchsuchen, missbräuchliche Verwendung sofort aufspüren können und dann mit Handlungsoptionen Unterstützung bieten. ${ }^{6}$ Einen anderen Ansatz wählen jene Anwendungen, die auf die Information von Nutzerlnnen zielen und anhand von Labels (Klassifizierungen) die Verständlichkeit der Datenschutzbestimmungen und Nutzungsbedingungen von Websites bewerten.

Ob solche Technologien des Vergessens wirklich funktionieren und adäquaten Schutz persönlicher Daten im Internet bieten, ist fraglich. Es mag möglich sein, personenbezogene Daten aus Suchmaschinen zu löschen, was nicht heißen muss, dass die Daten an ihrem Ursprungsort gelöscht werden. ${ }^{7}$ Die zeitliche Dynamik von Daten ermöglicht kontinuierliche Geschäftsmodelle, die Datenschutz an regelmäßige Zahlungen koppelt. ExpertInnen verweisen auf die augenscheinliche Konsequenz: exklusive Nutzung dieser Technologien durch zahlungsfähige Kundlnnen ${ }^{8}$ und Vernachlässigung eines solidarischen Modells für Datenschutz. Ob diesem Trend die Einführung von Datenschutzstandards und Maßnahmen zur Bewusstseinsbildung entgegenwirken, ist offen. Eine wichtige Frage bleibt, wie das Recht auf Vergessen anhand von Technologien des Vergessens zukünftig wirkungsvoll umgesetzt werden kann.

\section{Zitierte Literatur}

Mayer-Schönberger, V., 2009, Delete the virtue of forgetting in the digital age: Princeton Univ. Press.

Vervier, L., Schomakers, E.-M., Lidynia, C. und Ziefle, M., 2017, Perceptions of Digital Footprints and the Value of Privacy.

\footnotetext{
5 happytimes.ch/news/computer-technik/10897-snowhaze-macht-sie-im-internetunsichtbar-eth-studenten-entwickelten-sicherheits-app-fuers-smartphone.html.

6 experian.co.uk/consumer/identity.html.

7 Für Österreich gilt dazu Art. 17, Abs. 2 der DSGVO.

8 marketplace.org/2018/04/02/tech/erasing-your-digital-footprint-hard/.
} 


\section{Digitales Nudging und Demokratie}

\section{Zusammenfassung}

Nudges sind „Anstupser“ für verändertes Verhalten. Politisches Nudging umfasst das Anstoßen von Verhaltensänderungen zum Beispiel in Bereichen der gesünderen Ernährung, der Verringerung des Energieverbrauchs oder in der Abfallvermeidung. Ein zentraler Mechanismus sind die ,Voreinstellungen' hinsichtlich von Entscheidungen. Zum Beispiel ist in Ländern wie Österreich, in denen BürgerInnen der potentiellen Organspende im Todesfall aktiv widersprechen müssen, die Zahl der Organspender höher als in Ländern, in denen der Organspende aktiv zugestimmt werden muss. Wenn Obst in der Kantine auf Augenhöhe sichtbar ist, wird öfter zum Obst gegriffen. Andere Beispiele sind die Nutzung von Erkenntnissen der Verhaltensforschung zur Gestaltung von Erinnerungsschreiben für noch nicht eingereichte Steuererklärungen (vgl. Beispiele im Bericht der OECD 2017 und OECD 2019). Würden diese Schreiben einen Hinweis darauf enthalten, dass in der Nähe lebende Bürgerlnnen ihre Steuererklärung bereits eingereicht haben, so würde die Wirksamkeit der Schreiben deutlich steigen. Nudging wird mit Big-Data-Ansätzen kombiniert zu digitalem Big Nudging, wobei daran angeknüpft wird, dass mit Big Data riesige Datensätze analysiert werden, um individuelle Muster in Konsum, Gesundheitsverhalten und politischem Verhalten zu identifizieren. ${ }^{1}$ Im digitalen Raum beinhaltet Nudging digitale Informationen, Warnungen, Erinnerungen und Voreinstellungen bei der Auswahl von OnlineAngeboten. Mit Echtzeitdaten lässt sich unmittelbar erfassen und darstellen, wie das individuelle Verhalten im Vergleich zu anderen abweicht spiegelt damit sofort eine eventuelle Abweichung von sozialen Normen mit dem Ziel der (Selbst-)Anpassung der AdressatInnen an die Norm. Für die Befürworterlnnen ist Nudging die absichtsvolle Führung von Bürgerlnnen bei gleichzeitiger Aufrechterhaltung ihrer Wahlfreiheit und gilt als motivierende Steuerung, die statt Zwang oder Verboten eingesetzt werden kann. Die "Stupser" sind gerade so erfolgreich, da sie unbemerkt bleiben und somit zu weniger Konflikten führen als Gebote und Verbote. Zugleich schränken sie die Selbstbestimmung ein - und damit die Grundlage der Kontrolle über das eigenen Leben - und unterminieren Verfassungsrecht und Demokratie. Nudging wird zudem zur Beeinflussung von WählerInnen genutzt. $^{2}$ politisches Nudging:

das Anstoßen von

Verhaltensänderungen

\footnotetext{
1 welt.de/print/welt_kompakt/print_wissen/article154584139/Manipulation-20.html.

2 theguardian.com/commentisfree/2017/mar/06/big-data-cambridge-analyticademocracy.
} 
Debatten über politische Steuerungsinstrumente und ihren manipulativen versus unterstützenden Charakter

Nudging und Big Data

\section{Überblick zum Thema}

Nach Erkenntnissen der Verhaltensökonomie können Verhaltensänderungen vielfach durch sanfte Anstöße bzw. Anreize - englisch: Nudges herbeigeführt werden. Mittels solcher Nudges könne das Verhalten von Menschen auf vorhersagbare Weise beeinflusst werden, ohne dass dabei Verbote und Anordnungen notwendig wären (Thaler/Sunstein 2009). Charakteristisch für Nudging ist in der Theorie, dass es keinen offensichtlichen Zwangscharakter gibt und sich die Einzelnen dem Anreiz entziehen können („opting out“). Das setzt allerdings voraus, dass sie die Nudges bemerken, was in der Praxis und im digitalen Raum gerade oft nicht der Fall ist. Das Nudging-Konzept ist auf die Gestaltung (oder auch die Architektur und das Design) von Entscheidungssystemen ausgerichtet. Im Falle von Online-Portalen geht es z.B. darum, Aufmerksamkeit zu binden, indem Angebote für weitere Filme, Produkte oder Dienstleistungen automatisch erfolgen. In der Politik geht es um Verhalten, das als für das Individuum und/oder die Gesellschaft als „besser“ erachtet wird, wie zum Beispiel bei Anreizen zur Gesundheitsförderung.

2017 erhielt der Verhaltensökonom Richard Thaler den Wirtschaftsnobelpreis für den Ansatz des Nudgings, der auch neue Ansätze zur verhaltensbasierten Regulierung in der Politik ermöglicht. Nudges - als Steuerungsinstrumente verstanden - sind im Sinne Thalers Verhaltensstimuli, mit der die Freiheit des Individuums bewahrt wird und sich Autonomie und Wahlfreiheit sogar erhöhen würden. Dieses von Thaler selbst als „libertärer Paternalismus“ bezeichnete politische Konzept (Thaler/Sunstein 2003) hat umfangreiche Debatten über politische Steuerungsinstrumente und ihren manipulativen versus unterstützenden Charakter ausgelöst, wobei kritische Stimmen Nudging als Manipulationsinstrument auffassen.

In der heute zunehmenden Kombination von Nudging mit Big Data entstehen Instrumente des digitalen „Big Nudging“ (Helbing et al. 2015; vgl. Grafenstein et al. 2018) und damit eine Verhaltenssteuerung mit schwer absehbaren Konsequenzen. Big-Data-Techniken erweitern die Prognosefähigkeit von Organisationen direkt durch die sofortige Verfügbarkeit von Daten und indirekt dadurch, dass die zugrundeliegenden Modelle durch Tests und mehr Variablen permanent optimiert werden können. Big Data und digitales Nudging kombiniert, wie auch andere Formen gesellschaftlicher Steuerung, die Analyse von Verhalten mit Versuchen der Lenkung von Verhalten. Smart Meter, „intelligente“ Stromzähler, ermöglichen es, den Verbrauch von Strom datengestützt genau zu beobachten und damit auch, den Stromverbrauch durch Anreize für verschiedene Kundengruppen zu beeinflussen.

Big Data kann auch verwendet werden, um individuelle Verhaltensweisen umfassend $\mathrm{zu}$ verstehen, gegebenenfalls zu prognostizieren und mit Nudges im digitalen Raum zu beeinflussen. Unternehmen nutzen dies für ihr Marketing und staatliche Institutionen nutzen zunehmend die Kombination, um BürgerInnen zu Verhaltensänderungen zu bewegen. Mittlerweile werden die politischen Nudging-Strategien von der OECD erhoben 
und ausgewertet (OECD 2017), wobei sichtbar wird, dass immer mehr Regierungen diese Art der Verhaltenssteuerung in so unterschiedlichen Feldern wie VerbraucherInnenschutz, Bildung, Energie, ${ }^{3}$ Umwelt, Finanzen, Gesundheit und Arbeitsmarktpolitik anwenden. Die OECD bekam die meisten Anwendungsbeispiele aus UK, Australien, Kanada, Dänemark und Spanien. Die vergleichenden Auswertungen sind darauf gerichtet, den experimentellen Ansatz breiter als Politikinstrument zu nutzen.

Nudging wird auch in Wahlkämpfen genutzt, wenn auf der Grundlage von Prognosen, wer welche Partei wählen würde bzw. noch unentschieden ist, die jeweiligen potentiellen Wählerlnnen durch Nudging selektiv zur Wahl "genudged“ werden (siehe Thema „Microtargeting“).

Die digitalen Nudging-Anwendungen von globalen digitalen Plattformen werden stark wachsen, da die Erwartungen, damit Verhalten steuern zu können, für Unternehmensziele sehr attraktiv sind. Experimente mit personalisierten Preisen können von KonsumentInnen nur aufwändig nachgewiesen werden und eine personalisierte Werbung der Zukunft kann auf immer mehr Daten aufbauen. Das Nudging der KonsumentInnen im Kontext der digitalen Verhaltensüberwachung ist eine Frage des KonsumentInnen- und des Datenschutzes, während es im politischen Bereich weitgehender um Freiheitsrechte insgesamt geht. Im Bereich der Politik sind zentrale Fragen zum Verhältnis von Freiheitsrechten und evidenzbasierter Politik noch offen. Bei Nudging geht es um die Verhaltenssteuerung durch „Anstupsen“, wobei die Grenzen zur Manipulation von Verhalten fließend sind. Digitales Nudging wird von denen, die es als Steuerungsinstrument sehen und entwickeln, als eine zwar absichtsvolle Lenkung von Individuen gesehen, doch würde bei Nudging gleichzeitig die Wahlfreiheit bestehen bleiben, da das Prinzip mit motivierender Steuerung statt mit Zwang oder Verbot arbeiten würde. In der Politik könnten Nudges eine hohe Wirksamkeit zeigen und erzeugen dabei, verglichen mit Gesetzen oder Verordnungen, weniger Konflikte. Die Kritik bezieht sich darauf, dass Nudges eine Form der Bevormundung und Manipulation der Einzelnen darstellen, dass die Beeinflussung intransparent ist und die Nudges somit die individuelle Freiheit beschränken, ohne das klar wird, wer dafür die Verantwortung hat und wie die Einschränkungen legitimiert sind.

\section{Relevanz des Themas für das Parlament und für Österreich}

Mit dem Nudging im Kontext von Digitalisierung und Big Data sind Gefahren für die Freiheit der für die Demokratie konstitutiven freien BürgerInnen verbunden: Denn Verhaltensveränderungen werden gerade unterschwellig und unsichtbar initiiert, womit die Nudging-Akteure zu selbsternannten und häufig unerkannten Auswahl-DesignerInnen (die das „Choice Design“ und das „Default-setting“ entwerfen) oder Auswahl-ArchitektInnen (die die „choice architecture“ entwickeln) von wirtschaftlichen und politischen Ent-
Nudging in

Wahlkämpfen

Gefahren für die

Demokratie

\footnotetext{
3 Z.B. Verbrauchsfeedback durch Heizenergiesparkonten: umweltbundesamt.de/sites/default/files/medien/1410/publikationen/2017-08-22_texte_692017_nudgeansaetze_nach-konsum_0.pdf.
} 
Menschenwürde, Autonomie scheidungen werden, ohne als solche erkennbar zu sein und ohne dass diese Entscheidungsarchitekturen als politische Maßnahmen erscheinen.

Auf grundsätzlicher Ebene stellen sich Fragen nach der Menschenwürde, wenn über Big-Data-gestützte Verhaltensbeeinflussung Menschen als Datenquellen zu Zwecken der Steuerung durch Dritte benutzt werden, aber auch Fragen nach Handlungsautonomie und wie die Freiheit von Fremdbeeinflussung gewährleistet werden kann. Diese Fragen zu bearbeiten, wäre die Voraussetzung, um langfristig zu breit akzeptierten Anwendungen in unterschiedlichen Politikfeldern zu kommen. In Österreich wird Nudging in der Öffentlichkeit vielfältig diskutiert, ${ }^{4}$ jedoch gibt es keine Studien zu konkreten Anwendungen oder Voraussetzungen für die Anwendung von Nudging.

\section{Vorschlag weiteres Vorgehen}

Im Rahmen einer Langstudie wäre es sinnvoll, zunächst systematisch die aktuellen technologischen Entwicklungen und die internationalen Anwendungsgebiete zu identifizieren. Der internationale und vor allem der europäische Rechtsrahmen wäre daraufhin zu untersuchen, wie privatsphärenorientierte Prinzipien trotz der hohen globalen Dynamik durchgesetzt werden können (z.B. die Möglichkeit Standardvorgaben zu setzen und andere zu verbieten). Die zweite Ebene wäre es, partizipativ Innovationspfade, Infrastrukturen und Software-Ebenen zu analysieren, die individuelle Datenprofile effektiver dezentral schützen können. Dazu gehören Möglichkeiten einer automatischen Sichtbarbarmachung von Nudging, die es BürgerInnen ermöglichen, auf Grundlage ihres Grundrechts auf informationelle Selbstbestimmung zu entscheiden, ob und welche Formen der digitalen Verhaltenssteuerung sie aktiv unterstützen bzw. dulden.

\section{Zentrale weiterführende Quellen}

Grafenstein, M. v. et al., 2018, Nudging. Regulierung durch Big Data und Verhaltenswissenschaften. ABIDA - Assessing Big Data, Berlin, abida.de/sites/default/files/ABIDA-Gutachten_Nudging.pdf.

Helbing, D. et al., 2015, Das Digital Manifest, Spektrum der Wissenschaft, 5-39.

OECD, 2017, Behavioural Insights and Public Policy: Lessons from Around the World: OECD Publishing.

OECD, 2019, Tools and Ethics for Applied Behavioural Insights: The BASIC Toolkit, OECD Publishing.

Thaler, R. H. und Sunstein, C. R., 2003, Libertarian paternalism, American Economic Review 93(2), 175-179.

Thaler, R. H. und Sunstein, C. R., 2009, Nudge. Wie man kluge Entscheidungen anstößt, 4. Aufl., Berlin: Econ.

4 Siehe z.B. trend.at/wirtschaft/nudge-wie-menschen-8359578 (Ausgabe 4/2017); vben.at/wp-content/uploads/2016/10/102016-5.pdf. 


\section{Deepfakes - Perfekt gefälschte Bilder und Videos}

\section{Zusammenfassung}

Gefälschte Bilder sind nichts Neues. Aber die Fähigkeit Realität zu verzerren hat mit der Deepfake-Technologie einen signifikanten Sprung gemacht. Es ist mittlerweile relativ einfach möglich, Audio- und Video-Dateien von echt wirkenden Menschen zu erstellen, die Dinge sagen und tun, die sie nie gesagt oder getan haben. Dabei werden eigenständig lernende Algorithmen wie neurale Netzwerke mit Audio und Bildbearbeitungssoftware kombiniert. Das Ergebnis sind für den/die Laien/in nicht vom Original zu unterscheidende Fälschungen. Welche Risiken birgt das? Vor allem Videos, die als Medium bisher ein relativ hohes Vertrauen genießen, können für Rufschädigung, Erpressung oder Marktmanipulation missbraucht werden - mit eklatanten Folgen für Einzelne, Unternehmen und Gesellschaft. Potenzielle Gefahr für die Demokratie, sowie nationale und internationale Sicherheit könnte etwa von gefälschten Aufnahmen von Gewalt, Kriegserklärungen, Ankündigungen drohender Katastrophen oder Beweisen für das kriminelle Verhalten eines Staatsoberhaupts ausgehen. Auch Wahlbeeinflussung oder staatlicher, z.B. polizeilicher oder geheimdienstlicher Missbrauch der Technologie sind denkbar. Außerdem könnte die unkontrollierte Verbreitung von Deepfakes zu einem starken Verlust von Vertrauen in politische und mediale Institutionen führen.

\section{Überblick zum Thema}

Nachdem verändertes und gefälschtes Foto- und Videomaterial so alt ist wie die Aufnahmetechniken selbst, hat der Realismus und die Einfachheit des mit Künstlicher Intelligenz „gefakten“ Bildes und Tons eine neue Stufe erreicht. Es ist mittlerweile mit frei erhältlichen Apps und Programmen möglich, komplexes Material wie z.B. Gesicht und Sprache einer Person nachzuahmen und in für Laien/innen echt erscheinendes Videomaterial zu verwandeln. Solche „Deepfakes“ haben jüngst viel Aufmerksamkeit auf sich gezogen, z.B. durch komplett gefälschte Reden von Politikerlnnen ${ }^{1}$ oder gefälschte Pornographie mit berühmten Persönlichkeiten. ${ }^{2}$ Der Begriff Deepfake setzt sich dabei aus „deep learning“, einer Methode den Lernerfolg künstlicher neuronaler Netze zu optimieren, und „fake“, also Fälschung zusammen.

Bei früheren Fälschungen wurde beispielsweise das Gesicht einer Person aus vorhandenem Bildmaterial ausgeschnitten und über das Gesicht einer anderen montiert. Der Prozess war relativ aufwendig, die Ergebnisse insbesondere bei bewegten Bildern oft offensichtlich zweifelhaft. Fälschungen waren relativ leicht an Übergangsstellen oder an Beleuchtungswinkeln zu erkennen. Solche „face swaps“ sind mittlerweile in spielerischen
Künstliche Intelligenz kombiniert mit Audiound Bildbearbeitungssoftware

\footnotetext{
1 You Won't Believe What Obama Says In This Video! youtu.be/cQ54GDm1eL0.

2 Künstliche Intelligenz: Auf Fake News folgt Fake Porn, Die Zeit, zeit.de/digital/internet/2018-01/kuenstliche-intelligenz-deepfakes-porno-face-swap.
} 


\author{
Maschinelles Lernen: \\ Neuronale Netzwerke \\ trainieren sich \\ gegenseitig
}

perfekt gefälschte Mimik und Stimme

Erkennung von Fälschungen
Apps weitverbreitet und liefern nahezu ohne Verzögerung auf den ersten Blick gute Ergebnisse; ähnliche Apps generieren Fake-Video-Anrufe oder verändern den Körper in gewünschter Weise. ${ }^{3}$ Fälschungen, die mit Hilfe Künstlicher Intelligenz, größerer Rechenleistung und professioneller Software erzeugt werden, sind dagegen noch viel überzeugender, weil hier 3D-Computergrafikmodelle, z.B. des gefälschten Gesichts, von Grund auf generiert werden. Hier entstehen bereits die ersten Geschäftsmodelle, welche den Zugang zu professioneller Deepfake-Software und Rechenleistung stundenweise verkaufen. ${ }^{4}$

Oft kommen dabei zwei gegnerische neuronale Netzwerke zum Einsatz, die selbstständig voneinander lernen (generative adversary networks, GAN, vgl. Goodfellow/Pouget-Abadie et al. 2014). Einer dieser lernenden Algorithmen (Generator) wird mit mehreren Stunden vorhandenen Videomaterials mit dem Ziel trainiert, anhand von vielen Variablen möglichst genaue Kopien erzeugen zu können. Der zweite Algorithmus, der Diskriminator, wird dahingegen trainiert, die Ergebnisse des ersten vom Original zu unterscheiden. Der Generator versucht Ergebnisse, also z.B. das Modell eines sprechenden Menschen, zu erzeugen, die der Diskriminator nicht mehr unterscheiden kann. Dadurch nähern sich die gefakten Inhalte dem Original nach und nach immer weiter an.

Hat der erste Algorithmus alle nötigen biometrischen Parameter und Eigenheiten wie Mimik, Mundbewegungen und Sprache einer Person in verschiedenen Situationen erlernt, kann professionelle Audio- und Bildverarbeitungssoftware z.B. ein Gesicht perfekt digital replizieren und in ein beliebiges Video derselben oder einer anderen Person in hoher Qualität einfügen oder ein neues Video erzeugen. Da auch alle charakteristischen Stimmeigenschaften wie Frequenz, Intonation oder Pausen erlernt und digital repliziert werden, passen dann nicht nur die Lippenbewegungen perfekt zum vermeintlich Gesagten, sondern auch die Stimme selbst. Dafür reichen schon wenigen Minuten gesprochenen Materials.

Für den/die Laien/in nicht zu erkennen, haben ExpertInnen verschiedene Ansätze entwickelt, um gefälschtes Bildmaterial zu entlarven. Beispielsweise fehlt in künstlich erzeugten Videos oft das physiologisch wichtige Augenblinzeln, dies machen sich ForscherInnen zu Nutze, um wiederum lernende Algorithmen auf die Erkennung solcher Abnormalitäten zu trainieren (Li, Chang et al. 2018). Auch unsichtbare „Wasserzeichen“ die auf eine bestimmte Kamera zurückzuführen sind, sind in Planung, oder auch Blockchain basierte verifizierbare Zeitmarken. Lernende Algorithmen wurden auch benutzt, um Kunstfälschungen zu entlarven, da alle Charakteristika jedes Pinselstriches des Gesamtwerks eines/r Künstlers/in analysiert und Abweichungen erkannt werden können. In einem Fall produzierte ein 3D-Drucker auf Basis solcher Daten ein Portrait Rembrandts das alle

\footnotetext{
3 Gesichter spielerisch tauschen mit Apps: play.google.com/store/search?q=deepfake\&c=apps\&hl=de.

4 deepfakesweb.com.
} 
Charakteristika eines echten Rembrandts aufweist, eine künstliche Intelligenz würde dieses vermutlich als echt klassifizieren (Floridi 2018).

Offen ist dabei die Frage, wie schnell die Entwicklung von Fälschungstechnologie auf Erkennungen reagiert. Während 2018 fehlendes Augenblinzeln als unfehlbares Kennzeichen für Deepfakes galt, tauchten wenig später die ersten Videos mit Augenblinzeln auf. Der Wettlauf zwischen Entwicklerlnnen ist in vollem Gange und es ist nicht immer klar, wer gerade die Oberhand hat und auf welcher Seite er/sie steht. Derzeit gelten Blockchain-Technologien als hoffnungsvollster Weg die Authentizität von Videos zweifelsfrei zu dokumentieren (Hasan/Salah 2019), und KISysteme selbst werden zur Detektion von Fakes eingesetzt. Dass Künstliche Intelligenz auch zu solchen Zwecken genutzt wird, ist offensichtlich und verschiedene Formen der Kriminalität treten heute entweder schon auf oder sind in naher Zeit absehbar (King, Aggarwal et al. 2018, siehe auch Thema „Künstliche Intelligenz“).

In Bezug auf Deepfakes stehen momentan strafbare Handlungen gegen Personen im Vordergrund. Die oben beschriebenen gefälschten Bilder und Videos können nicht nur rufschädigend wirken und Belästigungen hervorrufen, sondern bei Betroffenen auch zu schweren psychologischen Auswirkungen führen, insbesondere falls einzelne Fälle von den Medien ausführlich aufgegriffen werden. Auch Erpressungen und Identitätsdiebstähle könnten sich häufen, was z.B. auch eklatante Auswirkungen auf Unternehmen haben könnte. Auch Marktmanipulationen, beispielsweise am Aktienmarkt sind mit gefakten Aussagen von CEOs denkbar.

Momentan sind vor allem Persönlichkeiten öffentlichen Lebens betroffen, da für einen guten Fake genug Trainingsmaterial in Form von Bildern und Videos im Internet verfügbar sein muss. Allerdings reichen für einen täuschend echten „faceswap“, also den Austausch eines Gesichts, schon wenige Hundert Bilder der betreffenden Person. Durch den allgegenwärtigen Gebrauch von Smartphones und die weitverbreitete Speicherung von Fotos in angreifbaren Clouds oder in Sozialen Netzwerken kann potenziell jedeR Nutzerln zum Opfer werden (siehe Thema „Cloud Computing“). Besonders Selfies eigenen sich gut als Trainingsmaterial für die Neuronalen Netzwerke. Die einfache Fälschbarkeit der Stimme könnte auch weitreichende Auswirkungen auf Nutzung und Missbrauch von sprachgesteuerten Geräte haben. Wenn sich Missbrauchsfälle insgesamt häufen, kann das zu einem gravierenden gesellschaftlichen Problem werden.

\section{Relevanz des Themas für das Parlament und für Österreich}

Demokratiegefährdend wird die Entwicklung dann, wenn nicht Prominente, wie z.B. Filmstars, Opfer von Deepfakes werden, sondern EntscheidungsträgerInnen und PolitikerInnen. Das könnte auch Risiken für die die nationale und internationale Sicherheit bergen (Chesney and Citron 2018). Gefälschtes Bildmaterial kann als digitaler Beweis für beliebige Situationen genutzt werden. Nach dem gleichen Prinzip wie „Fake News“ spielt die Authentizität eines Videos oft keine Rolle mehr, nachdem es mehrfach
Wettlauf zwischen

Entwicklerlnnen

Schäden für Personen und Unternehmen; Marktmanipulation

Rufschädigung,

Belästigung,

Einschüchterung,

Erpressung

potenzielle Gefahr für Demokratie und nationale Sicherheit 
Auswirkungen auf Wahlen, Schüren von

Unruhen

möglicher Verlust von Vertrauen in Institutionen über Social Media geteilt wurde (siehe Thema „Robojournalismus“, und „Microtargeting“). Aufwendige Dementi-Kampagnen binden dann nicht nur Ressourcen, sondern führen auch oft durch die erhöhte mediale Aufmerksamkeit zu einer noch weiteren Verbreitung der Fälschungen. Bislang war Österreich noch nicht stark von diesem Phänomen betroffen, doch in absehbarer Zeit könnte sich das ändern, es gibt jedenfalls keine Barriere die eine Ausbreitung in Österreich verhindern könnte.

Tatsächlich wird eine gefälschte Rede eines/r Kandidaten/in bei einer politischen Wahl, ob als solche identifiziert oder nicht, wahrscheinlich Auswirkungen auf seine/ihre Wählerlnnenschaft haben. Gefälschte Aufnahmen von Polizeigewalt, Kriegserklärungen, Ankündigungen drohender Katastrophen oder Beweise für das kriminelle Verhalten eines Staatsoberhaupts haben das Potenzial, unmittelbar zu sozialen Unruhen zu führen (EPRS 2018). Auch staatlicher, z.B. polizeilicher oder geheimdienstlicher Missbrauch der Technologie ist denkbar, etwa zur Beweismittelfälschung in illiberalen Regimen.

Ob aufwendig gefälschte Videos zum Massenphänomen werden, ist derzeit vielleicht noch fraglich, da die Technologie dafür aber reif und einfach verfügbar ist, steht dem wenig entgegen. Gesellschaftliches Bewusstsein für die nahezu perfekte Fälschbarkeit von Video- und Audiomaterialien zu schaffen, ist ein demokratiestärkender Ansatz. Aber auch das weit verbreitete Bewusstsein darüber könnte selbst zu einem gravierenden Problem werden, weil das ohnehin schon angeschlagene Vertrauen in öffentliche Institutionen ${ }^{5}$ und Medien dadurch noch weiter ausgehöhlt werden könnte. Wenn jedes Video ein Fake sein könnte, was und wem glaubt man dann?

\section{Vorschlag für weiteres Vorgehen}

Eine genaue Erhebung des technischen Ist-Zustandes und dessen Weiterentwicklungspotenzials wäre die Grundlage für eine tiefergreifende Abschätzung und Bewertung bisheriger sowie möglicher sozialer, politischer und wirtschaftlicher Folgen. Auf dieser wissenschaftlichen Basis könnten dann Empfehlungen für Maßnahmen zum gesetzlichen, institutionellen und organisatorischen Umgang mit Deepfakes erarbeitet werden. Vorzugsweise könnte das unter Einbindung von Stakeholdern, Expertlnnen und einer breiten Öffentlichkeit geschehen. Dieser vorausschauende Umgang mit dem Thema könnte dabei helfen, mögliche ernsthafte Schäden von Demokratie und öffentlichem Leben abzuwenden.

5 Standard Eurobarometer 88 - Public opinion in the European Union, ec.europa. eu/commfrontoffice/publicopinion/index.cfm/ResultDoc/download/DocumentKy/82873. 


\section{Zentrale weiterführende Quellen}

Chesney, B. and D. Citron (2018). Deep Fakes: A Looming Challenge for Privacy, Democracy, and National Security. University of Texas School of Law.

EPRS (2018). Global Trendometer. Essays on medium- and long-term global trends. Brussels, European Parliamentary Research Service European Parliament.

Floridi, L. (2018). Artificial Intelligence, Deepfakes and a Future of Ectypes. Philosophy \& Technology 31(3): 317-321.

Goodfellow, I., J. Pouget-Abadie, M. Mirza, B. Xu, D. Warde-Farley, S. Ozair, A. Courville and Y. Bengio (2014). Generative adversarial nets. Advances in neural information processing systems: 2672-2680.

King, T., N. Aggarwal, M. Taddeo and L. Floridi (2018). Artificial Intelligence Crime: An Interdisciplinary Analysis of Foreseeable Threats and Solutions, Oxford Internet Institute, University of Oxford.

Li, Y., et al.(2018). In Ictu Oculi: Exposing Al Generated Fake Face Videos by Detecting Eye Blinking. (preprint) arXiv:1806.02877."

Hasan, H. R. und Salah, K., 2019, Combating Deepfake Videos Using Blockchain and Smart Contracts, IEEE Access 7, 41596-41606. 


\section{Reallabore/Sandboxes als regulatorische Experimentierräume}

Als zentrale gesellschaftliche Herausforderungen erfordern Klimanotstand und der Wandel von Wirtschaft und Gesellschaft durch Digitalisierung und Dekarbonisierung dringliche Lösungen. Ohne eine Beschleunigung technologischer, sozialer und institutioneller Innovationen werden diese nicht rasch genug umgesetzt werden können. Daher sind neue Maßnahmen und missionsorientierte Politikinstrumente notwendig, die Akteursgruppen abgestimmtes Handeln in relevanten Politikfeldern ermöglichen und welche möglichst zu Win-win-Situationen führen. Zunehmend etablieren sich dafür Initiativen und Instrumente, die die Schaffung von Experimentierräumen ermöglichen, in denen Innovationsprozesse unter begünstigenden regulatorische Rahmenbedingungen beschleunigt werden können.

In Österreich wurden erste Aktivitäten gestartet, beispielsweise zur Umsetzung der Klima- und Energiestrategie durch das Programm Energie.Frei.Raum (BMNT), sowie in den Bereichen Digitalisierung (BMVIT) und FinTech (BMF). Solche Reallabore (im englischen Sprachraum wird häufig der Begriff „Regulatory Sandboxes“ verwendet) ermöglichen es, als regulatorische Experimentierräume innovative Lösungen, Technologien, Produkte, Dienstleistungen und Geschäftsmodelle für einen bestimmten Zeitraum und in einem kontrollierten Umfeld zu erproben. Dafür werden Ausnahmen von gesetzlichen oder regulatorischen Bestimmungen gewährt, die als Hemmnisse identifiziert wurden. Nach Beendigung der Experimentierphase werden diese gesetzlichen oder regulatorischen Freiräume bzw. Ausnahmen in der Regel wieder aufgehoben. Ziel von Reallaboren ist meist auch, in der Praxis erprobte, Erfahrungen für die zukünftige Weiterentwicklung des Rechtsrahmens zu sammeln.

Initiativen zur Umsetzung von Reallaboren sind ein vielversprechendes neues politisches Instrument, um Dekarbonisierung und Digitalisierung zu beschleunigen und chancenreich umzusetzen. Manche sprechen auch von einem Paradigmenwechsel beim Thema Innovation (BMWi 2019). Damit verbunden ist aber auch ein zusätzlicher Bedarf an Abstimmung und Vernetzung auf und zwischen mehreren Governance Ebenen: Einerseits auf der Ebene der Gesetzgebung, um rechtliche Voraussetzungen für das Experimentieren zu schaffen und Lernerfahrungen gegebenenfalls in neue Gesetze einfließen zu lassen; zweitens auf Ebene der Exekutive, um politikfeldübergreifende Innovationsunterstützung anbieten zu können; drittens auf Ebene der Akteure und Stakeholder, um Innovationsprozesse umzusetzen und Lernerfahrungen in die legistischen Prozesse zurückzuspielen.

Regulatorische Experimentier- und Freiräume sind vor allem in zwei Bereichen hoher Innovationsdynamik als neues Politikinstrument in Erscheinung getreten: Einerseits besteht Bedarf in Innovationsfeldern mit raschem technologischen Fortschritt, vor allem im Bereich der Digitalisierung. Inzwischen werden Reallabore international in Digitalisierungsbereichen wie
Schaffung von

Experimentierräumen

für Innovationsprozesse

\section{3 relevante}

Umsetzungsdimensione n: Gesetzgebung, Exekutive, Stakeholder 


\author{
Anwendung in den \\ Bereichen FinTech, \\ eHealth, Blockchain, \\ Sharing Economy
}

Ziel: effektivere Balance zwischen Regulierung und Innovationsförderung beispielsweise FinTech, eHealth, eGovernment, autonomes Fahren, Blockchain für das Internet der Dinge oder in der Sharing Economy angewendet. Hohe Innovationsdynamik trifft hier zum einen auf stark regulierte Sektoren, in denen - wie im Fall von Fintech - ein starker Wunsch der Innovatoren nach Deregulierung vorherrscht, und zum anderen auf Sektoren und Anwendungen, deren gesellschaftlichen Auswirkungen wenig vorhersehbar sind und für die noch keine gesetzlichen Rahmenbedingungen geschaffen wurden. In beiden Fällen hinken legislative Prozesse den realen Entwicklungen hinterher. Anregungen für die jüngsten Bestrebungen in Österreich, Regulatory Sandboxes im FinTech-Sektor zu etablieren, liefert das seit 2015 bestehende Programm der Britischen Finanzmarktaufsicht, UK Financial Conduct Authority. Deren Ziel ist es, dem raschen technologischen Fortschritt in der Finanzwirtschaft zu begegnen, sowie eine effektivere Balance zwischen Regulierung und Innovationsförderung zu schaffen. Andererseits besteht Bedarf in Bereichen in denen große gesellschaftliche Herausforderungen zu lösen sind. Hier legitimiert der dringliche Handlungsbedarf missionsorientierte Innovationspolitik. Dies gilt insbesondere für die Dekarbonisierung von Städten, Industrien, sowie der Energie- und Mobilitätssysteme. Die Urgenz wird hier durch die Ausrufung des Klimanotstands durch Städte, Länder bis zum Europäischen Parlament unterstrichen. Die Forderung gilt auch für die Bereiche Bildung, Gesundheit und Sozialpolitik.

Die angestrebte Transformation des Energiesystems und die Maximierung des Einsatzes von erneuerbaren Energien bei effizienter Nutzung von vorhandenen und neuen Infrastrukturen machen es erforderlich, die Flexibilität im Energiesystem verstärkt nutzbar zu machen, um Netzstabilität und Versorgungssicherheit gewährleisten zu können. In einem zunehmend dezentral funktionierenden Energiesystem macht dies Innovationen im institutionellen und regulatorischen Rahmen notwendig. International haben Länder wie Deutschland, Großbritannien, Niederlande, Norwegen und Singapur bereits solche regulatorischen Experimentierräume im Bereich der Smart Grids etabliert; Österreich ist gerade dabei sich diesem Trend anzuschließen. Andere Länder, wie Australien, Dänemark, Indien und Schweden, befassen sich ebenfalls mit derartigen Modellen. In Deutschland wurde für das Innovationsprogramm Schaufenster Intelligente Energie (SINTEG), auf Basis einer Ermächtigungsgrundlage im Energiewirtschaftsgesetz (EnWG) von der Bundesregierung der Gestaltungsspielraum einer Experimentierklausel genutzt und eine spezielle SINTEG Verordnung erlassen. In Großbritannien wird das Programm Innovation Link vom Energieregulator OFGEM angeboten, um gegebenenfalls auch Ausnahmeregelungen für Regulatory Sandboxes zu erlassen. In Italien werden vom Energieregulator ARERA zielgerichtet Ausnahmen in Bereichen gewährt und Projekte finanziert, in denen ein energiepolitisches Interesse identifiziert wurde (siehe Lo Schiavo et al., 2013). In den Niederlanden besteht seit einigen Jahren für Netzbetreiber und Energiegemeinschaften die Möglichkeit, um zeitlich limitierte Ausnahmen im Bereich der dezentralen Erzeugung erneuerbaren Stroms anzusuchen. Die rechtliche 
Basis bietet ein Dekret, „Crown decree for experiments with de-centralized renewable electricity generation“ (siehe Lammers et al., 2017).

Eine erste Analyse der existierenden Programme und Initiativen im Energiebereich (siehe ISGAN, 2019) legt nahe, dass es unterschiedliche Ansätze hinsichtlich Grad und Form der gesetzlichen Ausnahmetatbestände (Anpassung von Regulierungen oder Deregulierung) gibt. Sie weisen unterschiedliche Zugänge und Formate auf, sprechen verschiedene Akteure und Stakeholder an und gewähren Experimentierraum für unterschiedliche Themen und Innovationsbereiche (z.B. Sektorkopplung, Speicherlösungen, Flexibilität im Energiesystem, neue Tarif- und Geschäftsmodelle). Dementsprechend ist auch nicht zu erwarten, dass sich ein einheitliches Modell für das Design eines solchen Programms durchsetzen wird. Es besteht allerdings ein breiter Konsens über die Wichtigkeit und Notwendigkeit von wissenschaftlicher Begleitforschung und Ex-post Evaluierung als Teil der jeweiligen Initiativen. Weitere experimentelle Ansätze zu regulatorischen Freiräumen finden sich im Bereich sozialer Innovationen. Derzeit entwickelt man in mehreren Ländern Experimentierräume, um die Auswirkungen einer Umstellung des Wohlfahrtssystems auf Grundeinkommen zu testen. Das kürzlich abgeschlossene finnische Experiment mit Grundeinkommen wird gerade wissenschaftlich ausgewertet und evaluiert. Weitere Experimente mit Grundeinkommen findet man in den USA (z.B. Stockton in Kalifornien), sowie in Kanada mit einem inzwischen abgebrochenen Experiment in Ontario.

Bisher sind zu den hier erwähnten Reallabor-Programmen keine Erfahrungen aus wissenschaftlicher Begleitforschung oder ex-post Wirkungsanalysen verfügbar. Daher fehlen auch robuste vergleichende Befunde zu Vor- und Nachteilen unterschiedlicher Umsetzungsmodelle. Dies erschwert es, bestehende Modelle von Reallaboren im nationalen Kontext ohne ein gewisses Risiko zu replizieren. Es weist aber auf den Paradigmenwandel im Bereich Innovation hin und betont den Bedarf an neuen Instrumentarien für den Fortschritt missionsorientierter Innovationspolitik. Die Einführung von Reallaboren und das Schaffen regulatorischer Experimentier- und Freiräume stellt weltweit eine Weiterentwicklung des Instrumentenkoffers missionsorientierter, transformativer Innovationspolitik dar. Sie könnten auch in Österreich zukünftig einen sehr wichtigen Beitrag zur Umsetzung der vier formulierten Missionsthemen in der Klima- und Energiestrategie \#mission2030 - (a) Plus Energie Quartiere, (b) integrierte regionale Energiesysteme, (c) Break-Through-Technologien für die Industrie und (d) energieeffiziente Mobilitätssysteme der Zukunft - leisten (BMVIT 2019). Die gesetzlichen Grundlagen dafür müssen erst geschaffen werden, insofern der derzeitige gesetzliche Rahmen den zuständigen Behörden keinen Spielraum durch Ausnahmetatbestände zugesteht. Ein weiterer Aspekt in Bezug auf Beschleunigung von Innovation ist auch die Phase der Umsetzung Europäischer Rechtsmaterie in Österreichisches Recht, wie beispielsweise das "Clean energy for all Europeans package", die zwischen 2019 und 2021 zu erfolgen hat.
Hohes Potenzial im Energiebereich, aber auch im Bereich sozialer Innovation

Fehlende Erfahrung, Begleitforschung und Wirkungsanalysen 
Organisationsspezifisch e Eigenlogiken, Normen und Werte prägen die Umsetzung

Art und Weise der

Einbindung von Akteuren beeinflusst zukünftige Handlungsspielräume
Regulatorische Experimentierräume können auf vier Formen von Ausnahmetatbeständen beruhen. Eine Ausnahme von einer Verbotsvorschrift ermächtigt eine Behörde von den festgelegten gesetzlichen Regelungen abzuweichen. Bei Ausnahmen von einem Genehmigungstatbestand verzichtet der Gesetzgeber auf das Erfordernis einer Genehmigung, auf eine Vorgehensweise oder einen Nachweis, und erleichtert oder streicht damit für die Innovatoren Arbeitsschritte und bürokratische Wege. Der Gesetzgeber kann auch zwecks Erprobung auf Nachweis- und Ausstattungserfordernisse verzichten. Es können weiters auch Bündelungs- und Auffangtatbestände genutzt werden, um Abweichungen von Zuständigkeitsund Formvorschriften (z.B. zwischen Bund und Ländern) zu regeln (BMWi 2019).

Die erfolgreiche Steuerung eines komplexen Instruments wie Reallabore bedingt die Klärung von Fragen nach der Entwicklung von (Spiel-)Regeln sowie deren Durchsetzung und Kontrolle bei Aufbau, Betrieb, Weiterentwicklung und Nutzung der erzielten Ergebnisse. Jüngere Forschung aus dem Bereich der politikwissenschaftlichen Governance-Forschung weist darauf hin, dass in einem derartigen Zusammenhang organisationsspezifische Eigenlogiken, Normen, Werte, Regeln, Zielsetzungen, Anreizsysteme, Praktiken und Prozesse zu berücksichtigen sind (siehe Peters, 1998 und 2013). Dies trifft auch auf Regulierungsbehörden zu, deren Zielsetzungen weitestgehend in Zeiten festgelegt wurden, in denen mit ganz anderen gesellschaftlichen Herausforderungen (insbesondere Deregulierungen und Unbundling im Bereich der Energieversorgung und verteilung) umzugehen war.

Neben der stärkeren Ausrichtung innovationspolitischer Agenden auf die klima- und energiepolitischen Zielsetzungen könnten regulatorische Experimentierräume den Spielraum zur Unterstützung der Energiewende erhöhen. Ob die Vorgabe des rechtlichen Rahmens und die Entwicklung von geeigneten Governance-Strukturen dafür ausreichend, ist in Anbetracht der Unterschiedlichkeit der Interessen und Vielfalt der betroffenen und beteiligten Akteure fraglich. Vielmehr wäre es ratsam auf der Ebene der Umsetzung darauf zu achten in welcher Form Akteure in Auswahl, Durchführung und Begleitung von Reallaboren eingebunden werden, welche Pfadlogiken sich dadurch im Innovationsgeschehen ergeben und wie die zukünftigen Handlungsspielräume dadurch beeinflusst werden könnten. Ebenso ist zu berücksichtigen für welche Zeiträume und räumliche Ausdehnungen Ausnahmetatbestände gelten sollen. Weiters ist die Frage der Aufsicht zu klären - um mit Juvenal zu sprechen: „Wer wird die Wächter selbst bewachen?"; und, last but not least, ist die Ausgestaltung der Lernprozesse sowohl für die legistischen Akteure, als auch für die mit der Governance betrauten Stellen, von zentraler Bedeutung. 


\section{Zitierte Literatur}

BMVIT 2019: Umsetzungsplan zur Energieforschungsinitiative in der Klimaund Energiestrategie, BMVIT in Kooperation mit WKO und Klima- und Energiefonds. Version 1.0 zur Mission Innovation Week im Mai 2019. www.ikes-umsetzungsplan.at

BMWi (2019). Freiräume für Innovationen - Das Handbuch für Reallabore. Deutsches Bundesministerium für Wirtschaft und Energie bmwi.de/Redaktion/DE/Publikationen/Digitale-Welt/handbuch-fuerreallabore.html

ISGAN (2019): Innovative Regulatory Approaches with Focus on Experimental Sand-boxes: Smart Grid Case Studies. Casebook. International Smart Grid Action Network (ISGAN). May 2019, iea-isgan.org/wp-content/uploads/2019/05/IS-GAN-Casebook$\%$ E2\%80\%9CInnovative-Regulatory-Approaches-with-Focus-on-Experimental-Sandboxes\%E2\%80\%9D.pdf

Jenik, I., Lauer, K. (2017). Regulatory Sandboxes and Financial Inclusion. CGAP Working Paper

Lammers, I. and Diestelmeier, L. (2017): Experimenting with Law and Governance for Decentralized Electricity Systems: Adjusting Regulation to Reality? Sustainability 2017, 9(2), 212;

Peters, G. B. (1998). Managing Horizontal Government: The Politics of Coordination. Research Paper, Canadian Centre for Management Development.

Peters, G. B. (2013). "Toward policy coordination: alternatives to hierachy." Policy \& Politics 41(4): 569-584.

Lo Schiavo, L. et al. (2013). Changing the regulation for regulating the change: Innovation-driven regulatory developments for smart grids, smart metering and e-mobility in Italy, Energy Policy, dx.doi.org/10.1016/j.enpol.2013.02.022 


\section{Zukunft der Bewertungsplattformen: Online Reputationsmanagement}

\section{Zusammenfassung}

Bewertungsplattformen sind bereits allgegenwärtig: sei es bei der Wahl des Hausarztes, beim Online-Einkauf von Waren oder bei der Buchung einer Unterkunft. Für Unternehmen bieten Bewertungsplattformen großes Potenzial zum Online-Reputationsmanagement. Auch KonsumentInnen profitieren von öffentlich zugänglichen Bewertungen im Zuge ihrer Kaufentscheidungen. Doch was, wenn subjektive Werturteile zu Cybermobbing, Rufschädigung oder verzerrten Tatsachendarstellungen führen? Dieser Artikel geht dieser Frage nach, fasst wesentliche Pro- und ContraPunkte der Bewertungsplattformen zusammen und verweist auf notwendige staatliche Rahmenbedingungen um die gesellschaftliche "Grauzone" der Online Ratings transparent und fit für die Zukunft zu machen.

\section{Überblick zum Thema}

Neben den Produkteigenschaften und dem Preis sind Bewertungen im Internet bereits das drittwichtigste Kriterium für Kaufentscheidungen. ${ }^{1} \mathrm{Mehr}$ als $70 \%$ der Bevölkerung in Industrieländern haben bereits eine OnlineBewertung abgegeben und fast $50 \%$ überprüfen Online-Ratings anderer bevor sie etwas kaufen. In den Wirtschaftssektoren Unterhaltungselektronik und Tourismus spielen die digitalen Bewertungen eine besonders große Rolle. Nicht nur für die Bevölkerung sind Online-Ratings ein wesentliches Kriterium für Kaufentscheidungen, auch am B2B Markt werden Unternehmensakquisitionen mittlerweile durch Ratingplattformen unterstützt. ${ }^{2}$

Der Mechanismus, der den Bewertungsplattformen zugrunde liegt: durch Online-Ratings werden KundInnen auf Unternehmen, Produkte und Services aufmerksam. Unter dem Stichwort "Online Reputationsmanagement" bzw. auf Englisch „eWOM (electronic word of mouth)“ findet das Konzept Aufmerksamkeit in der Betriebswirtschaft, Psychologie und Kommunikationsforschung, während der breiter gefasste Ansatz der Mundpropaganda seit den 1960er Jahren von SoziologInnen diskutiert wird (Katz\&Lazarsfeld 1955). Huete-Alcocer (2017) geht sogar so weit und beschreibt eWOM als das einflussreichste informelle Medium unter KonsumentInnen, Unternehmen und der breiten Bevölkerung.

Soziale Netzwerke, Webseiten oder auch eigens dafür eingerichtete Onlineplattformen ermöglichen mittlerweile die Bewertung von nahezu allem und jedem. Bewertet werden in der Regel Hotels, Restaurants, Ferien-
Drittwichtigstes

Kriterium für

Kaufentscheidungen

eWOM - electronic word of mouth

Soziale Netzwerke, Webseiten, eigene Onlineplattformen

\footnotetext{
1 So die Studie „Trend Radar 2019“ Simon Kucher \& Partners (2019), die die Meinung von 6.375 Personen aus 23 Ländern zusammenfasst.

2 techcrunch.com/2019/07/23/trustradius-a-customer-generated-b2b-softwarereview-platform-raises- $12-5 \mathrm{~m} /$.
} 
Unterscheidungskriterien: Bewertungsgegenstand, Art der Bewertung, Geschäftsmodell der Plattform

Herausforderung: Manipulation von Bewertungsinhalten

Prüfverfahren: Meta-Bewertung wohnungen, Wellness- und Shoppingangebote (Tripadvisor, Yelp, booking. com, Airbnb, Amazon, Ebay), aber auch die Qualität von Arbeitsplätzen (Glassdoor, Kununu), Gesundheitsdienstleistungen (DocFinder, jameda), Fahrdiensten (Uber, mytaxi), sowie Berufs- (spickmich.de, Lernsieg) oder Persönlichkeitsprofilen (forthepeeple, Facebook) oder Livestreams (YY.com).

Bewertungsplattformen können je nach Bewertungsgegenstand, Art der Bewertung und Architektur bzw. Geschäftsmodell einer Plattform unterschieden werden. Die Bewertung kann quantitativ oder qualitativ erfolgen: während einfache Klicks bzw. Likes die Menge an positiver bzw. negativer Meinung fassen, können anhand offener Kommentare neue Themen eingebracht und Aspekte abseits der vorgegebenen Bewertungsdimensionen angesprochen werden. Zusätzliche Kommentar- und Chatfunktionen bzw. User-Foren können neue Möglichkeiten zum Dialog bieten und gestehen NutzerInnen in vielen Fällen eine aktive Rolle im Reputationsmanagement zu. Das Geschäftsmodell und die Plattformarchitektur entscheiden maßgeblich über den Informationszugang (integriert/eigenständig, offen/geschlossen, kostenlos/gebührenpflichtig, anonym/registrierungspflichtig), d.h. über die Rollen und Gestaltungsmöglichkeiten der Nutzerlnnen.

Ein Problemfeld von Online-Bewertungsplattformen ist die Manipulation von Bewertungsinhalten. Dazu zählen „geschönte Urteile“, bezahlte Positivurteile, gekaufte Klicks und die sogenannten „Fake News" - verzerrte bzw. falsche Tatsachenbeschreibungen. Der Fall des erfundenen Restaurants $^{3}$, das über ein Online-Bewertungsportal zum bestbewerteten Restaurants Londons wurde, ist nur ein Beispiel dafür, dass viele Bewertungen gefälscht sind. Neben Manipulation gibt es auch rationale Argumente, die die Bedeutung von Bewertungsinhalten in Frage stellen. Wissenschaftliche Studien machen auf den „Herdeneffekt" beim Online-Rating aufmerksam, der den starken Einfluss von ersten Ratings auf die darauf folgenden beschreibt (Lederrey\&West 2018). Eine weitere Beobachtung zeigt, dass in den meisten Fällen überwiegend positive Bewertungen abgegeben werden und die Summe der abgegebenen Bewertungen demnach kein repräsentatives Sample darstellt (Verhoef et al. 2014). Eine zentrale Frage, die sich daher im Zusammenhang mit Online-Ratings stellt, ist die nach der Qualität und Aussagekraft der gesammelten Daten.

Der Manipulation von Bewertungsinhalten wird anhand unterschiedlicher Prüfverfahren der Kampf angesagt ${ }^{4}$ : von technologischen Lösungen, die auf die Identifikation von automatisiert generierten Inhalten abzielen, bis hin zu nutzerbezogenen Lösungen, die auf das menschliche Urteilsvermögen bauen. Auch neue Methoden, wie die Meta-Bewertung von Bewertungsquellen (d.h. wie vertrauenswürdig ist die Quelle der abgegebenen Bewertung) werden getestet (Kim et al. 2019).

3 theshedatdulwich.com/

vice.com/de_at/article/434gqw/ich-habe-meine-gartenlaube-auf-tripadvisor-zumbesten-restaurant-der-stadt-gemacht.

4 Siehe dazu verbraucherzentrale.nrw/sites/default/files/201812/Bewertungen_Untersuchung_Marktwaechter.pdf. 


\section{Relevanz des Themas für das Parlament und für Österreich}

Mit der Einführung der App zur bundesweiten Online-Bewertung von Lehrpersonal und Schulen ${ }^{5}$ und dem Projekt Kultur-Token ${ }^{6}$ zur Belohnung von klimaschonendem Verhalten von BürgerInnen gewinnt das Thema Online-Bewertungen für Österreich an Relevanz.

Nachdem die privat entwickelte Lehrerlnnen-Bewertungs-App einen Tag auf dem Markt verfügbar war, wurde sie aufgrund der heftigen Kritik wieder deaktiviert. Ähnlich diesem Fall gab es in Deutschland vor einigen Jahren bereits Erfahrungen mit einer ähnlichen Bewertungs-App ${ }^{7}$, die am Ende der Debatte eingestellt wurde. Die Erfahrungen macht deutlich, dass bestehende Machtverhältnisse, z.B. die Bedenken wichtiger Betroffener (LehrerInnen, Gewerkschaften) und daraus folgende Konsequenzen, großen Einfluss bei der Einführung neuer, digitaler Kommunikationstechnologien haben und umgekehrt, neue Kommunikationswege (z.B. die zusätzliche Möglichkeit für Schülerlnnen ihr Lehrpersonal zu bewerten und Feedback abzugeben) oft mit gesellschaftlichen Machtverschiebungen einhergehen. ${ }^{8}$

Es existieren gegenwärtig vielfältige Empfehlungen und Lösungsansätze zur Herstellung von Fairness und Transparenz in digitaler Kommunikation. Beispielhaft kann die nachgewiesene Wirksamkeit von Nach-KrisenEntschuldigungen (Li 2019) und dialogischen Verfahren der Krisenkommunikation (du Plessis 2018) angeführt werden. Im medizinischen Bereich sollen zentrale ethische Prinzipien eine faire und transparente Online-Bewertungen ermöglichen: Patientenwohlfahrt, Patientenautonomie, ärztliche Fürsorge und soziale Gerechtigkeit stehen dabei im Fokus (Strech 2011). Auch der Österreichische Ethik-Rat für Public Relations stellt einen Leitfaden zur „Ethik in der Digitalen Kommunikation“ zur Verfügung ${ }^{9}$ und selbst die Kirche publizierte bereits 2002 päpstlichen Rat zur Ethik im Internet und verweist auf die Gefahr des Kulturimperialismus durch mangelnden Dialog. ${ }^{10}$ Doch inwieweit abstrakte ethische Prinzipien und Kommunikationsrichtlinien die Fairness in Online-Ratings garantieren können, sei dahingestellt; kritische Stimmen verweisen auf die hohen Ansprüche dialogischer Kommunikation, die v.a. im Dialog mit Regierungen und staatlichen Organisationen erhebliche Einschränkungen und Defizite aufweist (Lane 2018).

\footnotetext{
5 wienerzeitung.at/nachrichten/politik/oesterreich/2038228-Lehrer-BewertungsApp-startet.html.

6 digitales.wien.gv.at/site/kultur-token-projektinformation/.

7 de.wikipedia.org/wiki/Spickmich.

8 faz.net/aktuell/finanzen/meine-finanzen/geld-ausgeben/online-bewertungenwerden-fuer-unternehmen-wichtiger-15086974.html.

9 prethikrat.at/wp-content/uploads/2016/09/Kodex-Digitale-Kommunikation_PREthik-Rat.pdf.

10 vatican.va/roman_curia/pontifical_councils/pccs/documents/ rc_pc_pccs_doc_-̄20020228_ethics-internet_ge.html.
}

\author{
Relevanz für Österreich: \\ Lehrerlnnen- \\ Bewertungs-App und \\ Kultur-Token
}

\author{
Ethische Prinzipien \\ für die digitale \\ Kommunikation
}


Analyse der bisherigen Erfahrungen mit Online-Bewertung

Gütesiegel für faire und transparente Online-Bewertungen?
Gerade im Kontext neuer Entwicklungen, wie das Projekt Kultur-Token, dass Online Bewertungen zur Evaluierung klimaschonenden Verhaltens von BürgerInnen im öffentlichen Sektor einsetzt, wäre es wünschenswert ethische und gesellschaftsrelevante Fragestellungen vorab zu diskutieren. Fragen die sich stellen sind: Wie transparent ist das Verfahren zur Bewertung klimaschonenden Verhaltens von BürgerInnen? Welche Mitgestaltungsmöglichkeiten bieten sich im Rahmen des Anreizsystems „KulturToken" für Bürgerlnnen? Wie können algorithmische Diskriminierung und verzerrte Zielgruppen-Sampels in den Anwendungsfällen vermieden werden? Welchen Einfluss hat das zugrundeliegende Machtverhältnis StaatBürgerInnen auf die Bewertungsdynamik?

\section{Vorschlag weiteres Vorgehen}

Es gibt viele Erfahrungen mit Online-Bewertungsportalen in unterschiedlichen Anwendungskontexten und Ländern. Trotz vielfältigen Beispielen und unterschiedlichen Strategien zu transparenter und fairer OnlineKommunikation, ist das Feld sehr zerklüftet und es scheint keine einheitliche, in Beziehung gesetzte Linie für Bewertungen zu geben. In unterschiedlichen Bereichen machen sich Akteure für die Durchsetzung von Standards und Verhaltensregeln stark. Was bisher fehlt ist die Analyse und Ableitung von Schlussfolgerungen. Am Schluss ist klar, dass zukünftig nicht die Regulierung bzw. neue Gesetzgebung im Fokus der Debatte steht. Das grundlegende Recht auf Meinungsfreiheit, sowie strafrechtliche Tatbestände sind gesetzlich ausreichend geregelt, genauso Spezialfälle im Gesundheitsbereich (ärztliche Schweigepflicht). Generell gilt für digitale Kommunikation das gleiche Recht wie in der analogen Welt: Rufschädigung, üble Nachrede, etc. sind strafbar. Mehrebenen-Ansätze sollten daher vielmehr die Pflege der Kommunikationskultur in den Fokus rücken, wesentlich ist: es geht um die "Grauzone" die stark mit unterschiedlichen Vorstellungen von Dialog verknüpft ist und auf zwischenmenschlichen Umgangsformen aufbaut.

Was in Österreich für den digitalen Handel als E-Commerce Gütesiegel ${ }^{11}$ bereits verwirklicht ist, könnte auch aus Perspektive von Bürgerlnnen zu einem zivilgesellschaftlich vertretbaren Standard für die digitale Kommunikation im öffentlichen Sektor führen. Demnach wäre eine Möglichkeit für das Österreichische Parlament bisherige Erkenntnisse und Erfahrungen zu nutzen und einen Prozess anzuregen, der die kontinuierliche Reflexion digitaler Kommunikation in Hinblick auf Transparenz und Fairness ermöglicht. Um die Chancen und Risiken im pro-aktiven Umgang mit OnlineReputationsnetzwerken vor allem im staatlichen Bereich auszuloten, würde sich ein Foresight-Prozess in Hinblick auf zukünftige Anwendungsund Einsatzgebiete für den öffentlichen Sektor anbieten.

11 guetezeichen.at/. 


\section{Zitierte Literatur}

du Plessis, C., 2018, Social media crisis communication: Enhancing a discourse of renewal through dialogic content, Public Relations Review 44(5), 829-838.

Huete-Alcocer, N., 2017, A Literature Review of Word of Mouth and Electronic Word of Mouth: Implications for Consumer Behavior, Frontiers in psychology 8, 1256-1256; auch veröffentlicht in: Front Psychol.

Katz, E. und Lazarsfeld, P. F., 1955, Personal influence: the part played by people in the flow of mass communications, New York, NY, US: Free Press.

Kim, A., Moravec, P. L. und Dennis, A. R., 2019, Combating Fake News on Social Media with Source Ratings: The Effects of User and Expert Reputation Ratings, Journal of Management Information Systems 36(3), 931-968.

Lane, A. B., 2018, If it's so good, why not make them do it? Why true dialogue cannot be mandated, Public Relations Review 44(5), 656-666.

Lederrey, G. und West, R., 2018, When Sheep Shop: Measuring Herding Effects in Product Ratings with Natural Experiments, Proceedings of the 2018 World Wide Web Conference, Lyon, France: International World Wide Web Conferences Steering Committee.

Li, Y., 2019, Can an apology change after-crisis user attitude? The role of social media in online crisis management, Information Technology \&amp; People 32(4), 802-827.

Simon Kucher \& Partners, 2019, Trend Radar "Rating Economy". Global Survey.

Strech, D., 2011, Ethical Principles for Physician Rating Sites, J Med Internet Res 13(4), e113.

Verhoef, L. M., Van de Belt, T. H., Engelen, L. J., Schoonhoven, L. und Kool, R. B., 2014, Social Media and Rating Sites as Tools to Understanding Quality of Care: A Scoping Review, J Med Internet Res 16(2), e56. 


\section{Robojournalismus und digitalisierte Medien}

Nachrichteninhalte werden zunehmend über sozialen Netzwerke und mobile Geräte konsumiert. Das hat zur Folge, dass einige wenige digitale Plattformen die Verteilung der Nachrichten dominieren und von steigenden Werbeeinnahmen zu Lasten der traditionellen Nachrichtenunternehmen profitieren. Diese Nachrichtenunternehmen reagieren oft mit Sparmaßnahmen, wie der Reduktion von Angestellten und Inhalten, die zu weniger Breite und Qualität der Nachrichtenproduktion führen. Das wiederum vermindert die Attraktivität des Mediums für Leserlnnen und Anzeigenkundlnnen. Zusätzlichen Druck auf den Qualitätsjournalismus erzeugt die Automatisierung der Textproduktion, die bereits in einzelnen Sparten Realität ist, wie beispielsweise bei Börsen-, Wetter- oder Sportnachrichten.

Die Digitalisierung und Automatisierung der Medien hat weitreichende Folgen für das Berufsbild von Journalistlnnen und den Zugang zu verlässlichen Informationen für die Bevölkerung. Zunehmend wird der „klassische" Journalismus, der als Mittler Fakten überprüft und tiefgreifender recherchiert, aber auch Nadelöhr im Nachrichtenfluss ist, durch neue Formen und Akteure in Frage gestellt. Nachdem jede/r Besitzerln eines Smartphones potenziell über ein Ereignis berichten kann und über soziale Netzwerke oder offene Internetnachrichtendienste auch Zugang zu einer beträchtlichen Öffentlichkeit hat, fällt diese traditionelle Kontrollinstanz oftmals weg. Auf der einen Seite bedeutet dies eine Ermächtigung des Einzelnen, auf der anderen Seite leidet der informierte öffentliche Diskurs, wenn sich "Fake-News" einfach und schnell verbreiten und Algorithmen auf Meinung und Vorlieben einzelner Personen zugeschnittene Nachrichten automatisch produzieren und zustellen. Gleichzeitig ermöglicht der Digitalisierungstrend auch die Veränderung der journalistischen Arbeit hin in Richtung höhere Qualität (Stichwort „Datenjournalismus“ oder „Augmented Journalism"): mithilfe von automatisierten Analysetools, umfassenden, neuen Nachrichten-Datenbanken und künstlicher Intelligenz können zukünftig Inhalte schneller und besser aufbereitet werden. ${ }^{1}$

Soziale Netzwerke filtern Nachrichteninhalte mit intransparenten Mechanismen vor, die beispielsweise auf Likes oder Empfehlungen basieren. Dadurch konsumieren insbesondere Gruppen mit ähnlichen (politischen) Interessen verstärkt ausgewählte Medieninhalte; deren Meinungsbildung und Diskurs findet so vornehmlich unter Gleichgesinnten in ihrer „Filterblase" statt. Dadurch verändern sich die Wahrnehmung von Sachverhalten und die Einschätzung der Relevanz eines Themas. Ob Journalistlnnen in Zukunft vermehrt analysieren und kommentieren oder ob andere Szenarien den medialen Diskurs zu organisieren vorstellbar sind und was gesellschaftlich gefördert werden sollte, wäre Gegenstand einer wichtigen gesellschaftlichen und demokratiepolitischen Debatte. 1 insights.ap.org/uploads/images/the-future-of-augmented-journalism_ap-
report.pdf. 


\section{Zitierte Quellen}

Réchard, D. et al. (2017) Global Trendometer: Essays on medium- and longterm global trends. European Parliament, DOI:10.2861/782776, europarl.europa.eu/RegData/etudes/STUD/2017/603253/EPRS_STU( 2017)603253_EN.pdf.

Fanta, A. (2017) Putting Europe's Robots on the Map: Automated journalism in news agencies. Reuters Institute Fellowship Paper, University of Oxford, reutersinstitute.politics.ox.ac.uk/our-research/putting-europesrobots-map-automated-journalism-news-agencies 


\section{Microtargeting - Personalisierte Nachrichten zur Beeinflussung von Verhalten}

Microtargeting bezeichnet die gezielte Kommunikation mit Nachrichten, die auf bestimmte Charakteristika einer Person zugeschnittenen sind, um damit beispielsweise das Wahl- oder Kaufverhalten zu beeinflussen. Das kann in sozialen Netzwerken, Internetnachrichtenkanälen oder auch direkt an der Haustür geschehen. Durch die automatisierte Analyse großer Datensätze, wie z.B. Facebook-Profile und -Likes, lassen sich kleine, spezifische Personengruppen mit ähnlichen Persönlichkeitszügen (Psychogrammen) und sozio-demographischen Parametern finden. Diese Kleingruppen und sogar Einzelpersonen können dann mit auf sie abgestimmten (automatisiert) erstellten „Nachrichtenbeiträgen“ oder Werbeanzeigen beeinflusst werden. Die Inhalte bestehen oft aus stark selektierten Informationen bis hin zu frei erfundenen "Fake-News“. Dadurch verschwimmen die Grenzen zwischen Realität und Fiktion und es entstehen Informations-Filterblasen, die ein massives Problem für die Demokratie sind, da auf diese Weise freie Meinungsbildung und öffentlicher Diskurs untergraben werden können.

PolitikerInnen und ihre Wahlkampfteams setzen Microtargeting ein, um bei voraussichtlich knappem Wahlausgang Anhängerlnnen zu bestärken, Unentschiedene zu überzeugen, NichtwählerInnen zu motivieren oder GegnerInnen zu verunsichern. Während des Obama-Wahlkampfes 2008 wurde digitales Microtargeting erstmals bekannt und wird seitdem von beiden großen Parteien in den USA eingesetzt, ebenso wie von Parteien in Europa. Alle großen deutschen Parteien experimentieren etwa mit gezielter Facebook-Werbung. Eine großflächige Beeinflussung der Meinung von Wählerlnnen wird zwar in Frage gestellt, allerdings können diese Methoden das sprichwörtliche Zünglein an der Waage sein, das Wahlen entscheidet. Auch Firmen setzen Microtargeting ein, um mit Hilfe von BigData-Analysen spezifische Psychogramme zu erstellen, die zu erfolgreicheren Werbekampagnen führen können. Bedenkt man die enormen Umsätze, die Google und Facebook im letzten US-Kongresswahlkampf 2018 mit politischer Werbung erwirtschaftet haben (an die 430 Millionen USDollar), wird die Bedeutung des Geschäftsmodells ersichtlich. ${ }^{1}$

Die im Mai 2018 in Kraft getretene Datenschutzgrundverordnung (DSGVO) lässt Microtargeting in der EU, anders als etwa in den USA, nur unter sehr spezifischen Voraussetzungen zu. Wird die mutmaßliche politische Einstellung einer Person über Korrelationen anderer Daten bestimmt, bedarf dies der dezidierten Zustimmung. Wahlkampfteams statten ihre Helferlnnen aber beispielsweise mit Apps aus, die aufgrund kumulierter Daten die Wahrscheinlichkeit für eine bestimmte Wahlentscheidung anzei1 netzpolitik.org/2019/twitter-verbietet-politische-werbung-eine-herausforderung-
an-facebook/. 
gen. Hier wird zwar angegeben, dass direkter Personenbezug vermieden werde, beispielsweise durch die Bündelung einiger Adressen zu Wohnblocks oder Straßenzügen. Ob Gesetz und tatsächliche Praxis im datengestützten (Haustür-)Wahlkampf übereinstimmen, bleibt allerdings zu überprüfen. Außerdem sollte untersucht werden wie zugeschnittene (Wahl-)Werbung Meinungsbildung und die Bildung der (politischen) Öffentlichkeit beeinflusst.

\section{Zitierte Quellen}

Sonja Kind, Sebastian Weide (2017) Microtargeting: psychometrische Analyse mittels Big Data. Themenkurzprofil Nr. 18. Büro für TechnikfolgenAbschätzung beim Deutschen Bundestag (TAB), tab-beimbundestag.de/de/pdf/publikationen/themenprofile/Themenkurzprofil018.pdf.

Barbara Kolany-Raiser, Tristan Radtke (2018) Microtargeting - Gezielte Wähleransprache im Wahlkampf. Assessing Big Data: ABIDA-Dossier, abida.de/sites/default/files/16_Microtargeting.pdf. 


\section{Transparente Algorithmen - Wie lässt sich algorithmische Diskriminierung verhindern?}

Entscheidungen werden zunehmend durch Algorithmen vorbereitet oder automatisch getroffen. Dies gilt für selbstfahrende Autos, vernetzte Haushaltsgeräte, Entscheidungsprozesse im Gesundheitswesen oder die Mediennutzung. Auch Einkaufsempfehlungen durch digitale SprachAssistenten wie Alexa, Cortana und Co. sowie die Bewertung von Menschen in der Arbeitswelt, bei Versicherungen oder Banken beruhen auf algorithmischen Entscheidungssystemen und selbstlernenden Programmen. In vielen Fällen liefern Algorithmen die Grundlage für Entscheidungen, die von existenzieller Bedeutung sind. Wer einen Kredit erhält und zu welchen Konditionen dieser vergeben wird, ob die Aufnahme in eine Versicherung möglich ist und wie hoch die zu zahlende Prämie ist, gehört ebenso dazu wie Algorithmen, die vorschlagen, wer zu einem Vorstellungsgespräch eingeladen werden soll, befördert oder entlassen werden soll (vgl. Schaar 2017). Da das zentrale Merkmal der auf Big Data basierenden algorithmischen Steuerung die Klassifizierung ist, d.h. die Zuordnung von Datenelementen zu bestimmten Gruppen, ergibt sich ein hohes Diskriminierungspotential. Die Diskriminierung von Bewerberlnnen aufgrund von Geschlecht, ethnischer Zugehörigkeit oder Religion ist verboten, die Auswahl nach Zugehörigkeit in sozialen Netzwerken wie Facebook oder Linkedln dagegen nicht (Boyd et al. 2014). So greifen Algorithmen in den Alltag und die Selbstautonomie der Einzelnen ein, ohne dass diese die Möglichkeiten haben, die Entscheidungen nachzuvollziehen oder eben diesen Entscheidungen zu widersprechen. Algorithmische Systeme wenden ihre Entscheidungslogik konsistent auf alle Fälle an, unterliegen keiner subjektiven Verzerrung, diskriminieren damit auch konsistent, wie sich in Bezug auf Bewerbungen bereits zeigt (Boyd et al. 2014). Hinzu kommt, dass sich Algorithmen auf komplexe Situationen einstellen, also selbst im Wandel sind. ${ }^{1}$

Für einige Bereiche wie das Gesundheitswesen, die Versicherungsbranche oder auch selbstfahrende Autos stellen sich konkrete Fragen nach der Notwendigkeit neuer gesetzlicher Regelungen und Kontrollmöglichkeiten. Wenn Geschäftsmodelle in Zukunft stark auf algorithmischen Systemen beruhen, deren Funktion den Kern des Geschäftsmodells ausmachen, können Transparenz und Kontrolle im Widerspruch zur unternehmerischen Freiheit sowie der Eigentumsfreiheit im Hinblick auf die algorithmischen Systeme stehen. Die seit Mai geltende 2018 EU-Datenschutz-Grundverordnung kann einzelfallbezogene Transparenz, Nach-

\footnotetext{
1 So kann eine künstliche Intelligenz stereotype Werturteile entwickeln, wenn sie ihr Wissen aus repräsentativen Texten der Menschheit generiert, die eben diese Stereotype enthalten Caliskan, et al. (2017). Für eine Vielzahl von Beispielen, wie Meinungen und unhinterfragte Hypothesen in die in mathematischen Modelle eingebettet sind, siehe O'Neil (2016).
} 
vollziehbarkeit und Korrigierbarkeit von automatisierten Entscheidung ermöglichen, ist jedoch auf individuelle Rechte bezogen und eignet sich daher nicht für die Analyse und Regulierung von gruppenbezogenen, systematischen Diskriminierungsrisiken und soziotechnischer Risiken (vgl. Dreyer/Schulz 2017; Goodman/Flaxman 2017).

Dieser Technologiebereich hat eine hohe Innovationsdynamik, sodass ein analytischer und politischer Zugang zu algorithmischen Entscheidungsprozessen notwendig ist, der die zukünftigen Potentiale und disruptiven gesellschaftlichen Veränderungen von algorithmischen Entscheidungssystemen zur Grundlage nimmt. Auf grundlegender Ebene stellt sich die Frage, welche politischen Herausforderungen die intransparenten Systeme und das abzusehende Maschinenlernen aufwerfen und wie Transparenz, ethische Bewertungskriterien und demokratische Kontrolle gewährleistet werden können. Da sie auch in zentralen Infrastrukturen der Zukunft steuernd wirken werden, geht es darum, Möglichkeiten der Evaluierung, Transparenz und Kontrolle auch über die gesetzliche Regulierung hinaus zu untersuchen. Die diskriminierenden Folgen der Klassifikation durch Algorithmen stehen dem Diskriminierungsverbot entgegen und können verfassungsgesetzlich gewährleistete Rechte in hohem Maße tangieren. Eine vorausschauende Befassung muss insbesondere die internationale Dimension der Technologieentwicklung berücksichtigen, die nur begrenzt gesetzlich zu regeln ist.

\section{Zitierte Quellen}

Boyd, D., Levy, K. und Marwick, A., 2014, The networked nature of algorithmic discrimination, Data and discrimination: Collected essays.

Caliskan, A., Bryson, J. J. und Narayanan, A., 2017, Semantics derived automatically from language corpora contain human-like biases, Science 356(6334), 183-186.

Dreyer, S. und Schulz, W., 2017, Was bringt die Datenschutz-Grundverordnung für automatisierte Entscheidungssysteme? Potenziale und Grenzen der Absicherung individueller, gruppenbezogener und gesellschaftlicher Interessen, Gütersloh: Bertelsmann Stiftung.

Goodman, B. und Flaxman, S., 2017, European Union Regulations on Algorithmic Decision Making and a "Right to Explanation", Ai Magazine 38(3), 50-57.

ITA 2017, Roboter, Digitalisierung und Arbeitsmarkt. ITA-Dossier Nr. 26 (April 2017; Autorlnnen: Tanja Sinozic, Michael Nentwich, Johann Čas), Wien, epub.oeaw.ac.at/ita/ita-dossiers/ita-dossier026.pdf.

O'Neil, C., 2016, Weapons of math destruction: How big data increases inequality and threatens democracy: Crown.

Schaar, P., 2017, Überwachung, Algorithmen und Selbstbestimmung, in: bpb: Bundeszentrale für politische Bildung (Hg.): Digitale Gesellschaft und politisches Handeln, Bonn. 


\section{Staatliche Souveränität im digitalen Zeitalter}

Die Entwicklung zu einem Staat mit hoher Digitalisierung von Aufgaben und Infrastruktur ist seit Jahren ungebremst: Softwareproduzenten drängen mit immer neuen Angeboten auf den Markt, und Behörden suchen neuartige Lösungen zur Verwaltungsvereinfachung und Effizienzverbesserung. Mittlerweile werden staatliche Aufgaben und Dienstleistungen vielfach vollautomatisiert erbracht. Die erwarteten positiven Effekte reichen von Einsparungen bis hin zu neuen Daten als bessere Entscheidungsgrundlagen (Data Driven Government) und zur Minimierung von bürokratischem Aufwand (vom One-Stop- zum No-Stop-Government). Die Regierung bekennt sich zur weiteren Digitalisierung: Alle Bürgerlnnen sollen eine digitale Identität bekommen, mehr Behördenwege digitalisiert werden oder entfallen; durch Daten-Zusammenführung soll auch der Gesetzesvollzug profitieren, etwa im Bereich der Besteuerung. ${ }^{1}$

Doch wie verändert das alles unser Verständnis von „Staat"? Dies wird unter anderem unter dem Stichwort der digitalen Souveränität diskutiert (z.B. Müller Quade et al. 2018; BITKOM 2015). Durch Digitalisierung werden Bereiche des Staates auch unbeabsichtigt verändert (z.B. wer Zugang zu welchen Kommunikationskanälen hat ${ }^{2}$ ) und es eröffnen sich Räume, in denen Machtverhältnisse nicht klar geregelt sind. Kann das die Souveränität des Staates gefährden? Souveränität hieße hier Selbstbestimmtheit, die Fähigkeit eigenständig und unabhängig Entscheidungen (z.B. über Softwarelösungen und Datenhaltung) zu treffen. Dazu ist nicht unbedingt Autarkie erforderlich, aber Pfadabhängigkeit bei Informationstechnologie-Infrastrukturen, und nicht zuletzt Abhängigkeit von Monopolisten würde diese Souveränität gefährden. Es müssen ausreichend Kompetenzen vorhanden sein, verschiedene Lösungen zu verstehen, miteinander zu vergleichen, selbstständig zu betreiben und weiterzuentwickeln. Die Auslagerung hoheitlicher Aufgaben an privatwirtschaftliche, oft grenzüberschreitend agierende (und Daten auch anderswo speichernde) EDVAnbieter scheint problematisch, etwa in Cloudspeichern oder bei der Verwendung von Routern. Darüber hinaus entstehen durch die Digitalisierung der Verwaltung neue Sicherheitsprobleme und veränderte Herausforderungen für das Informationssicherheitsmanagement. Durch das Übertragen von Aufgaben an technische Systeme (z.B. Entscheidungssysteme auf Basis von $\mathrm{KI}$ ) werden viele von innen zu sog. Kritischen Infrastrukturen, deren Ausfall von wesentlicher Bedeutung für die Aufrechterhaltung staatlicher Funktionen wäre (Strauß/Krieger-Lamina 2017). Es wäre für den Staat zweckmäßig, sich auf den möglichen Ausfall dieser wichtigen Funktionen vorzubereiten und Überlegungen anzustellen, wie die Souve-

\footnotetext{
1 futurezone.at/netzpolitik/von-breitband-bis-egovernment-das-plant-dieregierung/302.588.659.

2 Vgl. etwa derstandard.at/2000078770407/IT-Blamage-Chat-desBundeskanzleramts-war-fuer-jeden-zugaenglich.
} 
ränität im Sinne von Kontrolle über hoheitliche Infrastrukturen aufrechterhalten werden könnte (siehe auch Nentwich et al. 2019).

\section{Zitierte Quellen}

BITKOM (2015), Digitale Souveränität. Positionsbestimmung und erste Handlungsempfehlungen für Deutschland und Europa, abgedruckt in: Datenschutz und Datensicherheit 2018 (5), 294-300.

Müller Quade, J., Beyerer, J. und Reussner, R. H., 2018, Karlsruher Thesen zur Digitalen Souveränität Europas, Datenschutz und Datensicherheit (5), 277-280.

Nentwich, M., Jäger, W., Embacher-Köhle, G. und Krieger-Lamina, J., 2019, Kann es eine digitale Souveränität Österreichs geben? Herausforderungen für den Staat in Zeiten der Digitalen Transformation. ITA Manu:scripts Nr. ITA-19-01 epub.oeaw.ac.at/ita/ita-manuscript/ita_19_01.pdf.

Strauß, Stefan, Krieger-Lamina, Jaro (2017): Digitaler Stillstand: Die Verletzlichkeit der digital vernetzten Gesellschaft - Kritische Infrastrukturen und Systemperspektiven, Projekt-Endbericht, Institut für Technikfolgen-Abschätzung: Wien, epub.oeaw.ac.at/ita/ita-projektberichte/2017-01.pdf. 


\section{Digitalisierung und Anonymität - Ein Widerspruch in sich?}

\section{Zusammenfassung}

Die Digitalisierung ist einer der wichtigsten Treiber wirtschaftlicher Entwicklung, sie stellt aber auch eine der großen Herausforderungen für Wirtschaft und Gesellschaft dar. Durch die zunehmende Verwendung digitaler Systeme entstehen unglaublich große Mengen von Daten in allen Anwendungsbereichen. Viele dieser Daten können zur Identifizierung von Personen verwendet werden. Damit ist eine anonyme Nutzung unterschiedlicher öffentlicher, aber auch privater (Kommunikations-)Räume nur mehr schwer bis gar nicht möglich.

Technische Entwicklungen, individuelles und gesellschaftliches Nutzungsverhalten sowie politische Rahmenbedingungen stellen Anonymität im öffentlichen Raum zunehmend in Frage. Anonymität stellt aber einen wesentlichen Faktor für freie Meinungsbildung, Entwicklung abweichender Verhaltensweisen und Gedanken als Kern gesellschaftlicher Entwicklung sowie für den Minderheitenschutz und somit für die Demokratie dar. Demokratie ist ohne Anonymität (in ihren unterschiedlichen Facetten von freien Wahlen, Berufsgruppenschutz von JournalistInnen (Kaye 2015), RechtsanwältInnen, PolitikerInnen, DiplomatInnen bis zu Sicherheitsexpertlnnen usw.) nicht möglich.

Technische Entwicklungskonzepte wie Privacy-by-Design, neue gesellschaftliche Vereinbarungen und Verhaltensweisen sowie politische Rahmenbedingungen können Bausteine dafür sein, Anonymität trotz allgegenwärtiger Digitalisierung zu sichern.

\section{Überblick zum Thema}

Im öffentlichen Raum verbringen wir einen wichtigen Teil unseres Lebens. Der Arbeits- oder Schulweg, das Treffen mit KollegInnen, Geschäftspartnerlnnen und Freundlnnen, Freizeitaktivitäten und Sport - alles spielt sich im öffentlichen Raum ab. Auch wenn wir uns damit nicht mehr im „privaten" Bereich, sondern in der Öffentlichkeit bewegen und so unsere Privatsphäre verlassen, konnten wir bisher davon ausgehen, dass auch und gerade in der Öffentlichkeit ein gewisses Maß an Anonymität gegeben war: Als Teil der Menge war man nicht identifizierbar, beobachtet werden konnte man immer nur von den ebenfalls Anwesenden und diese wiederum wechselten je nach Gelegenheit. Dies scheint sich zu ändern.

Der öffentliche Raum ist jedoch zentral für die Demokratie (BoehmeNeßler 2017). Der Meinungsaustausch am „Marktplatz“ gilt vielen noch heute als Synonym für gelebte Demokratie. Hier treffen Menschen unterschiedlichster sozialer Herkunft aufeinander, es ist der Raum des Miteinander und für Aushandlung und Kompromissfindung, hier werden fun-
Anonymität im öffentlichen Raum ist zentral für die Demokratie 
flächendeckende Überwachung

fortgeschrittene Gesichtserkennung damentale Rechte wie etwa Versammlungs- und Redefreiheit ausgeübt. Der öffentliche Raum bietet auch jenen eine Möglichkeit sich zu artikulieren, die nicht an vorderster Front ihre Ansichten zu vertreten gewohnt oder in der Lage sind. Die Anonymität ermöglicht Teilhabe, was zentral auch im verbrieften allgemeinen, gleichen, unmittelbaren, persönlichen, geheimen und freien Wahlrecht zum Ausdruck kommt. Dies gilt für alle Gesellschaftsmitglieder, aber insbesondere für Angehörige von Minderheiten sowie VertreterInnen abweichender Ansichten.

Schon heute ist in einigen Ländern eine nahezu flächendeckende Überwachung des öffentlichen Raumes gegeben. Diese stützt sich vor allem auf Videoüberwachungsanlagen, die sich zur Dokumentation und Expost-Feststellung von Vorgängen, wie etwa Unfällen oder auch kriminellen Taten, eignen. Die Videoaufnahmen konnten bisher nur einen Vorgang festhalten, nicht jedoch die Identität der aufgenommenen Personen offenbaren. Dies wurde erst mit dem Abgleich der Aufnahmen mit Bildern von gesuchten Personen möglich. Mit zunehmender technischer Reife der Kameras, der damit zusammenarbeitenden Analyse-Software und bestehender Bilddatenbanken wird dieses Instrument immer mehr zu einem Werkzeug für die direkte Identifizierung (Wordsworth 2017).

Die Verbindung von Biotechnologie und Informations- und Kommunikationstechnologien (IKT) führt zu fortgeschrittener Gesichtserkennung, die es in Zukunft möglich machen soll, aus DNA-Spuren auf wahrscheinliche Gesichter zu schließen und diese dann in einer Menge von Menschen zu identifizieren (siehe Thema "Gesichtserkennung“).

Die Gesichtserkennung wird immer besser und auch für private Nutzerlnnen möglich. Eine App für Smartphones ermöglicht es bereits, aus beliebigen, selbst geschossenen Fotos mit hoher Wahrscheinlichkeit die Identität einer fotografierten Person festzustellen, so diese Mitglied eines der großen sozialen Netzwerke ist (Wadhwa 2016). In diesen werden Milliarden von Fotos von anderen Personen hochgeladen. Die meisten der Abgebildeten hatten nicht die Möglichkeit, ihre Zustimmung dazu zu geben. Unseren „Gesichtsabdruck“ können wir nicht, wie etwa den Fingerabdruck mittels Handschuhen vermeiden. Niemand kann sich also mehr sicher sein, in der Öffentlichkeit anonym unterwegs zu sein. Es ist nicht nur das Gesicht, das identifiziert werden kann; wesentliche Entwicklungen gehen sogar dahin, mit Hilfe Künstlicher Intelligenz (KI) Menschen aufgrund ihres Ganges zu identifizieren (siehe auch Thema „Authentifizierung durch Verhalten“).

Der öffentliche Raum ist aber nicht nur der analoge, reale Raum. In modernen Gesellschaften spielen sich viele der oben angeführten Aktivitäten in virtuellen (Kommunikations-)Räumen ab. Diese sind umso stärker vom Verlust der Anonymität bedroht. Durch die Nutzung von Smart Devices und der Forderung immer „online“ zu sein, tragen wir auch selbst dazu bei, jederzeit ortbar und identifizierbar zu sein. Zu den Smart Devices zählen Smartphones, Navigationssysteme, Wearables mit Biosensoren für Sport und Lifestyle sowie medizinisch-technische Überwachungs- und Monitoringsysteme. Durch die fortschreitende Verbesserung bestehender 
Technologien wird sich die Überwachung noch wesentlich ausdehnen lassen. In naher Zukunft kennen intelligente Geräte routinemäßig die Bewegungen und den Standort einer Person bis auf zehn Zentimeter genau. Dies ermöglicht es, in die Aktivitäten, das Verhalten, die Interaktionen und die Beziehungen von Personen einzugreifen. Dadurch wird es für den/die Einzelnen immer schwieriger, unbemerkt zu bleiben. Die Einbindung von Personen in das Internet of Things (IoT) durch die Nutzung des noch engmaschigeren Mobilnetzes der 5. Generation (5G) verstärkt diesen Trend ebenfalls.

Durch den Einsatz von Nano- und Biosensoren, Gehirn-ComputerSchnittstellen und $\mathrm{KI}$ wird ständige Überwachung immer breiter und tiefer realisierbar. Zudem kommen auch Konzepte wie etwa Smart Cities nicht ohne die engmaschige Vernetzung und Auswertung der ungeheuren Datenmengen aus.

Die damit verbundenen positiven Erwartungen bzw. Versprechungen liegen vor allem in den Bereichen öffentliche Sicherheit, Umweltmonitoring und Gesundheit (Policy Horizons Canada 2014) sowie in den erwarteten Effizienzgewinnen und wirtschaftlichen Erfolgen (OECD 2017). Ob und inwieweit das Eingreifen von Sensoren und KI-gesteuerten Systemen im Notfall (z.B. Alarmierung bei schlechten Vitaldaten) jedoch die dauernde Überwachung rechtfertigen könnte, wäre zu diskutieren.

Insgesamt zeichnet sich eine Tendenz ab, Persönlichkeitsrechte und die grundrechtlich abgesicherte Privatsphäre zur Verhandlungsmasse unterschiedlicher Akteure zu machen (Policy Horizons Canada 2014). Dem Wesen von Anonymität als Grundpfeiler und Basis für Grundrechte wie Meinungs- und Versammlungsfreiheit wird dies aber nicht gerecht. Eher wäre zu fragen, wie der Prozess der Digitalisierung gestaltet werden kann, ohne die Möglichkeit zur Anonymität und damit zentrale Grundrechte zu verlieren?

\section{Relevanz des Themas für das Parlament und für Österreich}

Die Digitalisierung ist ein internationales Phänomen, hat freilich relevante Auswirkungen auf die Alltagswelt europäischer und österreichischer Bürgerlnnen. Wie die Gesellschaft ihre Demokratie gestaltet und welcher Einfluss den internationalen Konzernen auf politische Entwicklungen in der EU und Österreich zugestanden wird, könnte vom österreichischen Parlament beraten und mitgestaltet werden.

Es geht um grundlegende Fragen zum Menschenbild vom „freien Bürger“, der „freien Bürgerin“, den individuellen und gesellschaftlichen Auswirkungen der erodierenden Privatsphäre und dem möglichen Ende der Anonymität. Die Einflussnahme privater Konzerne auf das Such-, Informationsund Kommunikationsverhalten und letztlich das politische Verhalten der BürgerInnen beeinflusst die Gestalt unserer Demokratie.

Ein wesentlicher Aspekt in der Debatte um Anonymität und Demokratie ist die Gefahr der Ausblendung abweichender Gedanken und Verhaltens- positive Erwartungen

\author{
Wie Digitalisierung \\ gestalten, ohne \\ Möglichkeit zur \\ Anonymität und damit \\ zentrale Grundrechte zu \\ verlieren?
}




\author{
Richtlinien für \\ demokratiepolitisch \\ akzeptable, \\ privatsphären- \\ freundliche Produkte
}

weisen: Was in betriebswirtschaftlicher Logik Effizienzgewinne verspricht, kann langfristig zu einer Stagnation gesellschaftlicher Entwicklung in sozialen, kulturellen aber auch ökonomischen Bereichen werden. Ein subtiler Zwang zum Konformismus kann langfristig Demokratie und Wirtschaftssystem bedrohen (Tichy/Peissl 2001).

Vom Österreichischen Parlament könnten Impulse ausgehen, diese grundlegenden Fragen aufs Tapet zu bringen, bestimmte Businessmodelle zu hinterfragen und mittels Regulierung eventuell auch einzuschränken. Richtlinien für demokratiepolitisch akzeptable und privatsphärenfreundliche Produkte könnten Vorzeigecharakter für eine selbstbewusste europäische Politik in diesem Bereich sein.

\section{Vorschlag weiteres Vorgehen}

Eine mögliche Herangehensweise im Zuge einer Langstudie wäre es, zunächst einen breiten systematischen Überblick zu den aktuellen technologischen Entwicklungen und Anwendungsgebieten zu erstellen. Zudem wären der internationale und vor allem der europäische Rechtsrahmen zu untersuchen, um daraus abgeleitet Fragen zum Thema Anonymität und Demokratie im europäischen und nationalen Kontext zu diskutieren. Dazu gehören auch Fragen der Durchsetzung privatsphärenfreundlicher Prinzipen und Methoden in Forschung und Produktentwicklung.

\section{Zentrale weiterführende Quellen}

Boehme-Neßler, V., 2017, Videoüberwachung und Demokratie. heise.de/tp/features/Videoueberwachung-und-Demokratie3587282.html.

Kaye, D., 2015, Report of the Special Rapporteur on the promotion and protection of the right to freedom of opinion and expression, Human Rights Council: 21.

OECD, 2017, OECD Digital Economy Outlook 2017. Paris.

Policy Horizons Canada, 2014, MetaScan 3: Emerging Technologies. horizons.gc.ca/en/file/6210/download?token=g6Fb9RVA

Tichy, G. und W. Peissl, 2001, Beeinträchtigung der Privatsphäre in der Informationsgesellschaft. Grundrechte in der Informationsgesellschaft - 24.-26.5. Weißenbach am Attersee. Österr. Juristenkommisssion (ÖJK). Wien, Neuer wissenschaftlicher Verlag. 18: 22-48.

Wadhwa, T., 2016, Opinion: Powerful facial-recognition software can shred your privacy with just 1 photo. marketwatch.com/story/facialrecognition-will-soon-end-your-anonymity-2016-06-02.

Wordsworth, R., 2017, All eyes on you: what is the future of public surveillance? techradar.com/news/all-eyes-on-you-what-is-the-futureof-public-surveillance. 


\section{Technische Arbeitsplatzüberwachung}

\section{Zusammenfassung}

Überwachungstechnologien werden ständig weiterentwickelt und sind kostengünstig verfügbar, was eine Anwendung am Arbeitsplatz als Werkzeug für das Management, gerade in Zeiten vermehrter Telearbeit, plausibel erscheinen lässt. Die Arbeitsleistung von Menschen werden überwacht, etwa durch das Aufzeichnen von Mausbewegungen und Tastaturanschlägen, automatischen Screenshots, mittels GPS-Ortung, durch minütliches fotografieren per Webcam und dem Einsatz von Gesichtserkennungssoftware, durch das Auswerten der Leistung des Arbeitsgerätes oder mittels Algorithmen zur automatischen Berechnung der Produktivität. Während verhältnismäßiges Monitoring, z. B. durch Zeitaufzeichnungen oder Zutrittskarten berechtigt und legal ist, können andere Formen der Arbeitsplatzüberwachung Persönlichkeitsrechte und damit die Menschenwürde verletzen und führen mitunter zu vermehrten Arbeitsunfällen oder Produktivitätseinbußen. Gerade in Unternehmen mit schwachen oder nicht-vorhandenen Betriebsräten können solche Überwachungspraktiken schnell Einzug halten. Hier ist eine zeitgemäße Überprüfung bestehender Gesetze nötig.

\section{Überblick zum Thema}

Der Trend zu vermehrter Arbeit im Homeoffice hat sich auch schon vor der Corona-Krise abgezeichnet und wurde nun deutlich verstärkt. Viele Unternehmen hegten jahrelang Skepsis gegenüber der Telearbeit, wobei beispielsweise Produktivitätseinbrüche durch mangelnde Kontrolle und schlechte Kommunikationsmöglichkeiten befürchtet wurden. Nachdem jetzt sehr viele Arbeitnehmerlnnen monatelang zur Heimarbeit gezwungen waren, und viele Arbeitsabläufe trotzdem funktionieren, erkennen viele Unternehmen Vorteile, wie z. B. Einsparungen bei Büroräumen oder anderen Kosteneinsparungen. Es ist zu erwarten, dass auch in Zukunft deutlich mehr Arbeit sowie Aus- und Fortbildung zumindest teilweise in Heimarbeit stattfinden wird.

Allerdings sind nicht alle Arbeitsverhältnisse von Vertrauen geprägt, was beispielsweise die lange Geschichte elektronischer Arbeitsplatzüberwachung zeigt (OTA 1987). Das Bedürfnis nach Kontrolle auf Seiten des Managements gepaart mit kostengünstigen digitalen und anderen Überwachungstechnologien führte in den letzten Jahrzehnten zu deren vermehrten Entwicklung und Einsatz (Ajunwa et al. 2017). Ein Trend der durch die Entwicklung hin zu mehr Telearbeit noch verstärkt wird.
Kontrollverlust durch mehr Home-Office?

Mangelndes Vertrauen trifft leicht verfügbare Überwachungstechnologien 
Produktivitäts-Scores und periodische Webcamfotos

Auswertung privater Inhalte

„Klassische" Arbeitsplatzüberwachung

Psychometrie, Drogen- und Gentests

Effizienzsteigerung ...

... Stress, Angst und vermehrte Unfälle ...

... kontraproduktive Vermeidungsstrategien
Gerade bei der Verwendung firmeneigener Geräte ist die Versuchung groß Software zu installieren die die vermeintliche Arbeitsleistung eines Menschen überwacht. Etwa durch das Aufzeichnen von Mausbewegungen und Tastaturanschlägen, automatischen Screenshots, mittels GPS-Ortung, durch minütliches fotografieren per Webcam und dem Einsatz von Gesichtserkennungssoftware oder durch das Auswerten der Leistung eines Computers oder mittels Algorithmen zur automatischen Berechnung der Produktivität. ${ }^{1}$ Während der Browserverlauf etwa Aufschluss darüber gibt, welche privaten Dienste (email, soziale Netzwerke etc.) genutzt werden, können über die Aufzeichnung der Tastaturanschläge auch vormals private Inhalte überwacht werden

Auch jenseits des Home-Office ist die Überwachung des Arbeitsplatzes ein relevantes Thema. So werden beispielsweise in den USA Namensschilder mit eingebautem Mikrofon, Standortbestimmung und Bewegungssensor getestet; UPS stattete bereits 2009 seine Lastwägen mit über 200 Sensoren aus, um Fahrtgeschwindigkeit und Haltezeiten sowie viele andere Parameter aufzuzeichnen; Amazon misst die Effizienz seiner Lieferanten mittels obligatorischer Apps oder Tablets; einige Krankenhäuser überwachen wie oft sich Krankenschwestern die Hände waschen mittels elektronischer Plaketten an Uniformen (Ajunwa et al. 2017). Mit Hilfe von KI soll die automatische Auswertung von Überwachungsvideos im Produktionsbereich eingeführt werden, mit dem Ziel Arbeitsleistung zu überwachen, persönliche körperliche Ermüdung zu messen oder für Gesundheitsrisikobewertung und prozessbasierte Qualitätskontrolle (Luo et al. 2019).

Auch andere Formen der Überwachung sind bei fehlender oder schwacher Regulierung denkbar. So werden etwa in den USA Maßnahmen angewendet um ArbeitnehmerInnen zu kontrollieren, die tief in die Privatsphäre eingreifen, z. B. psychometrische Tests, Drogentests, Biometrie, Hintergrundüberprüfungen, Bonitätsprüfungen, aber auch Gentests und die Bewertung von Veranlagungen zu Gesundheitsrisiken (Ajunwa et al. 2017).

Das Fehlen von verhältnismäßigem Monitoring von Arbeitsplätzen kann zu organisatorischen Abweichungen und Fehlverhalten führen. Elektronische Überwachung soll hier zu Effizienzsteigerung führen. Unverhältnismäßige Überwachung kann allerdings auch kontraproduktiven Stress und Angst hervorrufen. Werden z. B. gesetzte Ziele elektronisch überwacht, beeilen sich Arbeitnehmerlnnnen oft mehr, woraufhin es häufiger zu Verletzungen in Folge von Unfällen kommt (Ajunwa et al. 2017). Außerdem werden auch Anreize gesetzt „das System zu schlagen“. Es konnte beispielsweise gezeigt werden, dass Mitarbeiterlnnen, die Videoüberwachung als nötigend empfinden, sich Verhaltensweisen angewöhnen, um unsichtbar und unbemerkt zu bleiben, was auf Managementseite dann als Begründung für noch mehr Überwachung angeführt wird - ein Teufelskreis (Anteby/Chan 2018).

1 trendingtopics.at/ueberwachung-software-home-office-tools-grenzen/. 
Während verhältnismäßige Formen der Arbeitsplatzüberwachung, wie Zeitaufzeichnungen oder Zutrittskontrollen per Firmenausweis legal sind, befinden sich andere in regulatorischen Grauzonen, wieder andere sind zumindest in der EU - klar illegal. Prinzipiell ist Videoüberwachung von Mitarbeiterlnnen laut Datenschutzgesetz verboten, also auch die Nutzung der Webcam für diesen Zweck. Ausnahmen davon gibt es nur, wenn ein berechtigtes Interesse des Arbeitgebers am Einsatz eines Videoüberwachungssystems besteht. Dies kann beispielsweise im Schutz vor bzw. in der Hilfe zur Aufklärung von Diebstählen oder Überfällen liegen, etwa im Schalterraum einer Bank, wo ja primär nicht Arbeitnehmerlnnen beobachtet werden sollen. Soweit Arbeitnehmerlnnen in das Blickfeld der Kamera kommen ist jedenfalls die Menschenwürde berührt und die individuelle Zustimmung der Arbeitnehmerlnnen oder, wo einer besteht, durch den Betriebsrat in einer Betriebsvereinbarung, notwendig.

Das Verbot gilt natürlich insbesondere auch, wenn im privaten Bereich gearbeitet wird. Videocalls fallen nicht unter diese Regelung, wenn der Zweck eine Besprechung und nicht die Kontrolle ist. Allerdings können auch hier die Persönlichkeitsrechte dritter, betriebsfremder Personen verletzt werden, wenn z. B. Familienmitglieder mitaufgenommen werden. Die Verhältnismäßigkeit der Überwachungsmaßnahme muss also stimmen, was natürlich einen gewissen Graubereich mit sich bringt.

Für die algorithmische Berechnung von Produktivitäts-Scores zur Bewertung von Arbeitnehmerlnnen (auch bei Profilen und Prognosen) beispielsweise gilt die DSGVO und eine Datenschutz-Folgenabschätzung ist nötig. Hier müssen Arbeitnehmerlnnen bzw. Betriebsräte zustimmen, bzw. haben Vetorechte. Generell gilt, dass Überwachungsmaßnahmen die die Menschenwürde verletzen verboten sind, z. B. heimliches Abhören von Telefongesprächen, Überwachungskameras in Waschräumen oder Toilettenanlagen, oder die Überprüfung des Privatlebens. Überwachungsmaßnahmen die die Menschenwürde berühren, d. h. die Persönlichkeitsrechte der Arbeitnehmer entsprechend tangieren dürfen nur dann eingesetzt werden, wenn zwischen Betriebsrat und Unternehmen eine dementsprechende Betriebsvereinbarung getroffen wurde, oder MitarbeiterInnen den Maßnahmen einzeln schriftlich zugestimmt haben. ${ }^{2}$ Bei schwachen Betriebsräten oder deren gänzlichem Fehlen, z. B. in KMUs, oder bei ausreichendem Druck von Arbeitgeberlnnen auf einzelne Arbeitnehmerlnnen können also legal Maßnahmen eingesetzt werden, die deren Menschenwürde massiv berühren können.
Webcam im

privaten Bereich

Verletzung von

Persönlichkeitsrechten Dritter

Betriebsrat und MitarbeiterInnen mit Vetorecht ...

... kann zum

Schwachpunkt werden

2 arbeiterkammer.at/beratung/arbeitundrecht/Arbeitsklima/

Big_Brother_am_Arbeitsplatz.html. 

Rechtliche Grundlagen
überprüfen

\section{Relevanz des Themas für das Parlament und für Österreich}

Für Kontrollen in der Heimarbeit gelten dieselben Arbeits- wie Datenschutzgesetze wie am Arbeitsplatz im Betrieb, also Arbeitsverfassungsgesetz (ArbVG), Arbeitsvertragsrechtsanpassungsgesetz (AVRAG) und Europäische Datenschutz-Grundverordnung (DSGVO), sowie das Datenschutzgesetz (DSG). Hier stellt sich die Frage inwieweit diese Gesetze auch absehbare Fortschritte bei der Entwicklung von Überwachungstechnologien abdecken, und inwieweit Betriebsvereinbarungen oder die Zustimmung von Arbeitnehmerlnnen in Zukunft noch adäquate Werkzeuge sind.

\section{Vorschlag weiteres Vorgehen}

Eine vertiefenden Foresight- und TA-Studie könnte hier vertieft neueste Entwicklungen auf dem Überwachungstechnologiemarkt erörtern und vorausschauend Folgen von deren potentieller Anwendung im österreichisch/ europäischen Kontext abschätzen. Literaturrecherche mit ExpertInnenbefragungen sollten hier eine Grundlage an Daten liefern, auf der dann aufbauend unter Einbeziehung relevanter Stakeholder Folgen erörtert und insbesondere auch Empfehlungen für die Anpassung rechtlicher Rahmenbedingungen erarbeitet werden.

\section{Zitierte Literatur}

Ajunwa, I., Crawford, K. und Schultz, J., 2017, Limitless worker surveillance, Calif. L. Rev. 105, 735.

Anteby, M. und Chan, C. K., 2018, A Self-Fulfilling Cycle of Coercive Surveillance: Workers' Invisibility Practices and Managerial Justification, Organization Science 29(2), 247-263, doi.org/10.1287/orsc.2017.1175.

Luo, X., Li, H., Yang, X., Yu, Y. und Cao, D., 2019, Capturing and Understanding Workers' Activities in Far-Field Surveillance Videos with Deep Action Recognition and Bayesian Nonparametric Learning, Computer-Aided Civil and Infrastructure Engineering 34(4), 333-351, onlinelibrary.wiley.com/doi/abs/10.1111/mice.12419.

OTA, 1987, The Electronic Supervisor: New Technology, New Tensions, U.S. Government Printing Office, Washington, DC: , September 1987: Office of Technology Assessmen, U.S. Congress, ota.fas.org/reports/8708.pdf. 


\section{Pandemiemanagement mit technologischer Hilfe}

Die Stopp-Corona App war eine der ersten technologischen Anwendungen, die bei der Eindämmung der COVID-19-Pandemie helfen sollte. Sie war sehr schnell bekannt, aber in puncto Datensicherheit umstritten und wurde mit den Applikationen anderer Länder verglichen. Sie ist jedoch bei weitem nicht die einzige technologische Möglichkeit, die zur Pandemiebekämpfung genutzt werden kann.

International werden Drohnen immer häufiger eingesetzt. Während der Ausgangssperren in China wurden Lebensmittel und Medikamente auf dem Luftweg in das gesperrte Gebiet geliefert (siehe Lieferdrohnen). Drohnen werden vielerorts zur fliegenden Überwachung und zum Bewegungs-Monitoring eingesetzt. Personen ohne Mund-Nasen-Schutz werden mit Hilfe von Drohnen zum Tragen desselben ermahnt, außerdem werden Körpertemperaturen gemessen und Fotos gemacht. Per Gesichtserkennung werden Personen identifiziert und mit Datenbanken von Reiserückkehrern und unter Quarantäne gestellten Personen verglichen, woraufhin gegebenenfalls die Polizei verständigt wird. Diese Anwendungen sind zurecht umstritten und werden oft durch die spezielle und nur temporäre Situation der Pandemie begründet. Es ist wichtig zu bedenken, dass selbst wenn solche Maßnahmen in einer Ausnahmesituation als gerechtfertigt angesehen werden könnten, die Ausnahmesituation auch eine solche bleiben muss und Regulierungen für Drohnen-gestützte Überwachung notwendig sind.

Weniger problematisch aus datenschutzrechtlicher Sicht sind Drohnen, die Desinfektionsmittel versprühen und Roboter, die Viren und Bakterien mittels UV-Licht abtöten. ${ }^{1}$ Diese werden beispielsweise in Krankenhäusern genutzt, sodass kein Mensch die Zimmer von Infizierten betreten muss. Alle Aufgaben zusammengefasst sollen in Zukunft dedizierte Pandemie-Drohnen erledigen können. ${ }^{2}$ Solche Anwendungen sorgen für erleichterte und das Ansteckungsrisiko reduzierende Arbeitsbedingungen, können aber auch zu Stellenabbau führen, beispielsweise von Reinigungskräften.

Für die Identifikation vermeintlich infizierter Personen mittels der Daten verschiedener Sensoren gibt es bereits Anwendungen Künstlicher Intelligenz (KI). KI wird außerdem auf Social Media zur Erkennung von Fake News im Zusammenhang mit Covid-19 genutzt sowie zur Erkennung von Molekülen und zur Vorhersage neuer Mutationen (STOA 2020). Seit dem weltweiten Ausbruch des Virus werden weitere technologische Anwendungen zur Bekämpfung entwickelt. Neben den bereits genannten sind synthetische Biologie, Genetik und Nano-Technologien vielversprechend

\footnotetext{
1 Beispielsweise uvd-robots.com, die bereits weltweit eingesetzt werden.

27 news.com.au/lifestyle/health-wellbeing/coronavirus-drones-developed-byuniversity-of-south-australia-researchers-c-763510.
} 
(STOA 2020). Manche technischen Lösungen mögen unproblematisch sein, andere bergen eklatante Risiken für Demokratie und Menschenrechte. Diese sollten bei betreffenden Maßnahmen im Rahmen der Kontrollfunktion des Parlaments genau untersucht und abgewogen werden.

\section{Zitierte Literatur}

STOA, 2020, Ten technologies to fight coronavirus. European Parliament Research Service, Scientific Foresight Unit. PE 641.543. April 2020, europarl.europa.eu/RegData/etudes/IDAN/2020/641543/EPRS_IDA(20 20)641543_EN.pdf. 


\section{Exoskelette: Von digitalen Kampfanzügen zur futuristischen Gehhilfe}

Exoskelette sind am Körper tragbare Maschinen, die Menschen bei Bewegungen unterstützen und körperliche Belastungen reduzieren. Seit den 1960er-Jahren wird an der Entwicklung dieser Roboteranzüge gearbeitet. Exoskelette dienen der Stabilisierung des Bewegungsapparats, können aber auch vorhandene menschliche Fähigkeiten - wie Hebekraft - verstärken. Ursprünglich wurde die Technologie für militärische Zwecke entwickelt (z. B. um Bomben in Flugzeuge einzuladen), gegenwärtig spielt sie aber vor allem im medizinisch-therapeutischen Bereich und in der manuellen Industriearbeit eine Rolle (Bogue 2015). REX ${ }^{1}, \mathrm{HAL}^{2}$ und ReWalk ${ }^{3}$ sind gegenwärtig am Markt erhältliche Exoskelette, die für Therapie und Rehabilitation eingesetzt werden; der "Chairless Chair" ${ }^{4}$ oder die „ExoHand" 5 sind Beispiele für Exoskelette in der Industrie; auch in EU-Projekten wird an modularen Exoskeletten für die Industrie geforscht. ${ }^{6}$

Roboteranzüge und -geräte gelten als entscheidende Technologie, wenn es darum geht, die Eigenständigkeit älterer Menschen zu fördern (Bogue 2015).I Zentrum der Forschung steht hier beispielsweise die Rehabilitation der unteren Gliedmaßen, beispielsweise bei Lähmungen (Bao et al., 2019) Aber auch für die manuelle Arbeit in der industriellen Produktion haben Exoskelette eine wesentliche Bedeutung: sie können die Leistungsfähigkeit und Flexibilität von ArbeiterInnen erhöhen, indem mechanische Lasten reduziert werden und damit wiederkehrende Belastungen und Arbeitsunfälle vorgebeugt werden können (Bogue 2018). Industrielle Anwendungen von Roboteranzügen sind Prognosen folgend der vielversprechendste Zukunftsmarkt: mit einer durchschnittlichen jährlichen Wachstumsrate von $39 \%$ würde das für 2025 ein Marktvolumen von 1,9 Milliarden Dollar bedeuten. ${ }^{7}$

Ethische Überlegungen betreffen vor allem die hohen Anschaffungskosten von Exoskeletten (besonders bei der medizinischen Nutzung von Exoskeletten im Heimbereich) und die daraus resultierenden ungleichen bzw. exklusiven Zugangsmöglichkeiten zur Technologie (Bissolotti et al. 2018). Darüber hinaus sind Nebenwirkungen (Schmerzen, Unfallrisiko und nichterfüllte Erwartungshaltungen mit psychischen Folgewirkungen) bekannt, die mit dem therapeutischen Einsatz von Exoskeletten einhergehen können

\footnotetext{
1 rexbionics.com.

2 cyberdyne.jp/english/.

3 rewalk.com/de/.

4 noonee.com.

5 festo.com/group/de/cms/10233.htm.

6 robo-mate.eu.

7 abiresearch.com/press/abi-research-predicts-robotic-exoskeleton-market-e/.
} 
(Bissolotti et al. 2018). Auch Probleme, die durch die Reduktion menschlicher Interaktion entstehen, werden als kritisch erachtet (Bogue 2013).

Aus einer gesellschaftspolitischen Perspektive stellt sich einerseits die Frage, wie Exoskelette als hochtechnologische, medizinische Therapiegeräte im Kontext des demografischen Wandels zur Verfügung gestellt werden können, um ein hohes Maß an Selbständigkeit für ältere Menschen zu gewährleisten. Darüber hinaus eröffnet die Anwendung von Roboteranzügen in der industriellen Produktion ein Spannungsfeld zwischen individueller Arbeitssicherheit und -gesundheit einerseits und Überwachung bzw. Einschränkung der Privatsphäre am Arbeitsplatz andererseits. ${ }^{8}$ Welche zukünftigen Herausforderungen Exoskelette in Kombination mit neuen Technologien, wie z. B. neuronalen Schnittstellen oder haptischen Sensoren (zur Wärme- und Druckübertragung), mit sich bringen, sollte weiterverfolgt werden.

\section{Zitierte Literatur}

Bao, G., Pan, L., Fang, H., Wu, X., Yu, H., Cai, S., Wan, Y. (2019). Academic Review and Perspectives on Robotic Exoskeletons. IEEE Transactions on Neural Systems and Rehabilitation Engineering, 27(11), 2294-2304. doi:10.1109/TNSRE.2019.2944655

Bissolotti, L., Nicoli, F. und Picozzi, M., 2018, Domestic Use of the Exoskeleton for Gait Training in Patients with Spinal Cord Injuries: Ethical Dilemmas in Clinical Practice, Frontiers in neuroscience 12, 78-78.

Bogue, R., 2013, Robots to aid the disabled and the elderly, Industrial Robot: the international journal of robotics research and application $40(6)$, 519-524.

Bogue, R., 2015, Robotic exoskeletons: a review of recent progress, Industrial Robot: the international journal of robotics research and application 42(1), 5-10.

Bogue, R., 2018, Exoskeletons - a review of industrial applications, Industrial Robot: the international journal of robotics research and application 45(5), 585-590.

8 So werden beispielsweise in automatisierten Industrie-4.0-Anlagen zu Zwecken der Arbeitssicherheit vollüberwachte, digitale Zwillinge von ArbeiterInnen erstellt, die neben der Ortung am Betriebsgelände auch das Monitoring von individuellen Gesundheitsdaten (Puls, Bewegungsabläufe etc.) ermöglichen. 


\section{Chips der Zukunft: Elektronische Haut}

Elastische Chips, die wie ein Abzieh-Tattoo mit Wasser auf die Haut aufgeklebt werden, können Puls, Sauerstoffsättigung oder Hirnströme messen. Sie werden als „elektronische/künstliche Haut“ bezeichnet. Die mikroskopisch kleinen, elektronischen Schaltkreise können bspw. auf dem Handrücken oder an der Stirn angebracht werden, sind widerstandsfähig und stören ihre TrägerInnen kaum. Damit sind sie für den Einsatz im Sport, aber auch in der nicht-invasiven Medizin geeignet. Dort können sie in Verbindung mit entsprechenden Sensoren lebenswichtige Funktionen (Herzfrequenz, Sauerstoffkonzentration im Blut, etc.) erfassen und über ein Display mit Leuchtdioden auf der Haut anzeigen (Yokota et al. 2016). Bisher können erst einzelne Buchstaben oder Ziffern angezeigt werden. Zukünftig könnte die Entwicklung auch im Arbeitsbereich für die Anzeige von z. B. Bauplänen oder Diagrammen am Arm genutzt werden, ohne die TrägerInnen in ihrer Arbeit einzuschränken (DerStandard 2016).

Elektronische Haut hat ein hohes Zukunftspotential in Hinblick auf ihre Weiterentwicklung und Anwendung. Zur Aussicht stehen dabei die Entwicklung von umfassenden Displays und die Erforschung von neuen Einsatzgebieten (z. B. ganzheitliches Gesundheitsmonitoring durch Pflaster). Fortschritte bei der Anwendung elektronischer Haut in der Mensch-Maschine-Interaktion könnten zukünftig zu einer verbesserten Steuerung von Computern ${ }^{1}$ und virtuellen Objekten führen (Cañón Bermúdez et al. 2018). Ebenso wird an der Recyclierbarkeit bzw. Selbstreparaturfähigkeit der sogenannten "e-skin“ gearbeitet (Zou et al. 2018). Auch der Einsatz von künstlicher Haut für z. B. Armprothesen, um zukünftig Druckempfindung und das Fühlen von Wärme und Kälte zu ermöglichen (Ober 2015; Tee et al. 2015, Zhu et al., 2020) ist ein vielversprechender Anwendungskontext der gerade für Österreich mit innovationsstarken Akteuren in der Prothetik interessant erscheint.

\section{Zitierte Literatur}

Cañón Bermúdez, G. S., Karnaushenko, D. D., Karnaushenko, D., Lebanov, A., Bischoff, L., Kaltenbrunner, M., Fassbender, J., Schmidt, O. G. und Makarov, D., 2018, Magnetosensitive e-skins with directional perception for augmented reality, Science Advances 4(1), eaao2623.

DerStandard, 2016, Elektronische Haut verwandelt Handrücken in ein Display; Der Standard vom 17. April 2016, derstandard.at/2000035044646/Elektronische-Haut-verwandelt-dieHand-in-ein-Display.

\footnotetext{
1 handelsblatt.com/technik/it-internet/neue-sensortechnik-mit-elektronischer-hautden-computer-steuern/20878530.html?ticket=ST-1680141rgQ4Wjs9oSFRVFqyvOBo-ap4.
} 
Yokota, T., Zalar, P., Kaltenbrunner, M., Jinno, H., Matsuhisa, N., Kitanosako, H., Tachibana, Y., Yukita, W., Koizumi, M. und Someya, T., 2016, Ultraflexible organic photonic skin, Science advances 2(4), e1501856.

Zhu, M., Ali, M. U., Zou, C., Xie, W., Li, S., \& Meng, H. (2020). Tactile and temperature sensors based on organic transistors: Towards e-skin fabrication. Frontiers of Physics, 16(1), 1-13.

Zou, Z., Zhu, C., Li, Y., Lei, X., Zhang, W. und Xiao, J., 2018, Rehealable, fully recyclable, and malleable electronic skin enabled by dynamic covalent thermoset nanocomposite, Science Advances 4(2), eaaq0508. 


\section{Personalisierte Genomsequenzierung}

Individuelle Genomsequenzierung, wie sie heute schon möglich ist, erzeugt eine Karte der eigenen DNA, bei der der exakte Aufbau des gesamten Erbguts ausgelesen wird. Das kann medizinischem Fachpersonal dabei helfen, wirksame und sichere Behandlungen auf die persönlichen Bedürfnisse von PatientInnen abzustimmen. Zum Beispiel könnten so Dosierungen von Arzneimitteln und auch die Wahl der Medikamente selbst, besser auf den Stoffwechsel der jeweiligen Person angepasst und Nebenwirkungen vermieden werden. Preiswerte Diagnostik und bessere Prävention könnten auch Kosten im Gesundheitssystem senken.

Das Wissen über genetische Marker von Krankheiten wächst und dadurch wird das persönliche DNA-Profil in Diagnostik und Prädiagnostik nützlicher. Aufgrund der DNA-Daten können Medizinerlnnen bei Gesunden Aussagen darüber treffen, wie hoch das Risiko ist, an einer bestimmten Krankheit zu erkranken und das bereits lange vor dem Auftreten von Symptomen. Einige chronische Krankheiten können durch pränatale Interventionen, Frühgeburtenbehandlungen oder möglicherweise durch Gentherapie verhindert oder sogar ausgerottet werden. Das Auslesen des eigenen Genoms allein ist allerdings oft nur bedingt aussagekräftig, da das Wissen darüber, welche Genkombinationen oder welche Veränderung welche Krankheiten bedingen, noch lückenhaft ist. Vor allem aber bleiben Risikoeinschätzungen für viele Krankheiten Wahrscheinlichkeitsaussagen, auch wenn sie auf Basis des eigenen Genoms berechnet werden. Die Diagnose einer Erkrankungswahrscheinlichkeit kann aber bereits gravierende physische, psychische und soziale Folgen haben. Entscheidungen über Therapien können bei uneindeutigen Aussagen sehr schwierig sein, diese müssen die PatientInnen aber selbst treffen. Die präventiven Maßnahmen, die aus solchen Risikoeinschätzungen abgeleitet werden bewegen sich sehr oft im Bereich von allgemeingültigen Gesundheitsratschlägen, wie nicht zu rauchen, sich viel zu bewegen und gesund zu essen.

Die Technik der Sequenzierung ist ausgereift und im Wesentlichen beeinflussen nur logistische Fragen den Preis, damit ist Massenanwendung denkbar geworden. Unternehmen wie My Heritage, 23andMe, ancestry oder iGENA bieten über das Internet personalisierte DNA-Sequenzierung zur Untersuchung der eigenen Herkunft an. Der Kunde schickt dazu normalerweise eine Speichelprobe an das Unternehmen, das die Probe dann sequenziert, die Ergebnisse mittels verschiedener Modelle auswertet und dann an den Kunden zurückschickt. Die Aussagekraft der Tests ist aber umstritten, ${ }^{1}$ in Frankreich sind sie sogar verboten. Das Verbot wird aller1 spiegel.de/kultur/gesellschaft/ahnenforschung-dna-esoterik-zum-sonderpreis-
kolumne-a-1194396.html. 
dings nicht durchgesetzt und im Kontext der COVID-19-Pandemie kontrovers diskutiert. ${ }^{2}$

Brennende Fragen betreffen vor allem den Datenschutz, da aus einem sequenzierten Genom hochsensible Daten, wie Krankheitsrisiko oder Abstammung abgelesen werden können. So wird zum Beispiel Diskriminierung durch Arbeitgeber oder Versicherungen aufgrund genetischer Daten, wenn auch verboten, denkbar (siehe Gläserne Patient). Genetische Daten sind gemäß der DSGVO als „sensible Daten“ klassifiziert; für sie muss eine explizite Einwilligung eingeholt werden. In Österreich wird die Verarbeitung von genetischen Informationen weiters vom Gentechnikgesetz ${ }^{3}$ geregelt. Arbeitgebern und Versicherungen ist es in Österreich verboten, genetische Daten abzufragen. Die Weitergabe von Daten aus genetischen Analysen zur Untersuchung erworbener, d.h. nicht erblicher Mutationen an Versicherungen - nicht jedoch an Arbeitgeber - ist vom Verbot ausgenommen. ${ }^{4}$

2 connexionfrance.com/French-news/Health/French-DNA-testing-ban-is-affectingcoronavirus-research.

3 BGBI. 510/1994, IV. Abschnitt - Genetische Analysen und Gentherapie am Menschen.

4 gesundheit.gv.at/labor/untersuchungen/gentests/was-ist-das. 


\section{Automatisiertes Gesundheitsdaten-Monitoring}

In Zeiten demographischen Wandels, einem Mangel an Pflegekräften und dem Trend zu einer stärker selbstbestimmten Gesundheitsversorgung, steigt die Nachfrage nach teilautomatisierten Gesundheitsdienstleistungen. Ein Monitoring von Gesundheitsdaten, gekoppelt mit digital vernetzten Gesundheitsdiensten und Gesundheitseinrichtungen, kann zu einem teil-automatisierten Gesundheitssystem führen. Es könnte Menschen ermöglichen eigenständiger, trotz (chronischer) Krankheiten, zu leben und selbstbestimmter Gesundheitsvorsorge zu betreiben. Mit den Potentialen sind zugleich neue Risiken verbunden, da in der Medizin und im öffentlichen Gesundheitswesen immer mehr sensible PatientInnendaten digital gespeichert werden. In den kommenden Jahren wird mit einem starken Anwachsen personenbezogener Daten im Gesundheitswesen zu rechnen sein, ohne dass bisher in ähnlichem Ausmaß die steigenden Anforderungen an den Datenschutz in der Medizin bedacht werden.

Mit privaten, online angebotenen Genom-Analysen (siehe auch Thema „Personalisierte Genomsequenzierung“) geben weltweit nicht nur Erwachsene zunehmend sensible Gesundheits-Daten an private Anbieter weiter, sondern auch die Daten ihrer Kinder. Genetische Analysen am Menschen werden in Österreich durch das Gentechnikgesetz (GTG) geregelt, das neben der Zulassung von Einrichtungen, die prädiktive genetische Tests durchführen, auch Aufklärung, Einverständniserklärung, Beratung, Dokumentation und Datenschutz im Zusammenhang mit genetischen Analysen regelt. Da die privaten Anbieterlnnen, deren Dienstleistungen keinen klinischen Kriterien entsprechen müssen und auch nicht mit einer Beratung über die Reichweite der möglichen Ergebnisse verbunden sind, aus den USA ihre Leistungen anbieten, greift das GTG nicht. Die privaten AnbieterInnen koppeln die Genomanalyse auch mit Daten der NutzerInnen wodurch ein wachsender Austausch von sensiblen digitalen Gesundheitsdaten in privaten Datennetzen außerhalb Österreichs und der EU stattfindet. Zeitgleich wächst die Tendenz zum Dokumentieren und Teilen von Vitalund Aktivitätsdaten zur eigenen Gesundheits-Optimierung mittels HandyApps und Messgeräten, die mit Smartphones gekoppelt sind. In den möglichen Kombinationen von genetischen Daten, medizinischen Daten, dem umfassenden Monitoring des Lebensstils und der Kontrolle dieser Daten durch das Social Web ergeben sich langfristige Missbrauchspotentiale, die sich denjenigen kaum erschließen lassen, die heute ihre Daten teilen. 


\section{Bionische Produktion der Zukunft: Selbstformende Objekte durch 4D-Druck}

4D-Druck fügt dem 3D-Druck die Veränderung der Objekte durch äußere Faktoren hinzu. Smarte Materialien verwandeln sich eigenständig (aber zuvor präzise programmiert) in einen neuen Zustand. Objekte, im 3D-Druck aus verschiedenen Materialien zusammengestellt, sind so konzipiert, dass sie unter bestimmten Bedingungen ihre Gestalt ändern (Kuang et al., 2019). Dabei orientiert sich Forschung an bionischen Prinzipien der Natur, insbesondere dem Wachstum von Pflanzen oder auch den Schutzmechanismen von Pflanzen in Bezug auf Wasserzufuhr oder Temperaturveränderung.

Die Versprechungen sind hoch, reichen von neuen Implantaten (die minimalinvasiv in den Körper eingeführt werden und erst am Bestimmungsort ihre vollständige Größe erreichen) bis zu Möbeln, die sich zu Hause selbst zusammensetzen. Angedacht werden bioabbaubare Produkte aus Materialien, die sich nach einer gewissen Zeit oder auf entsprechende Impulse hin selbstständig zersetzen. Den unterschiedlichen Anwendungen ist gemeinsam, dass sie ein weitgehend neues Produktionsparadigma anstreben, das voraussichtlich insbesondere dann von schnell steigender Bedeutung sein wird, wenn es aus verschiedenen Branchen und Wissenschaftszweigen mit hoher Synergie vorangetrieben wird. Der 4D-Druck ist insofern primär interessant im Zusammenspiel mit persönlichen Fertigungseinheiten (personal manufacturing), mit Robotik und dem Internet of Things (Glenn/Florescu 2015).

Dem 4D-Druck wird eine potentiell disruptive Innovationsdynamik zugeschrieben. Branchenübergreifend haben die Technologien potentiell eine hohe Innovationsdynamik und sind daher von hoher wirtschaftlicher Relevanz. Wie der 3D-Druck bereits medizinische Produktherstellung, z. B. von Orthesen und in der Zahnmedizin disruptiv verändert und in Zukunft die Schmuckherstellung und die Verarbeitung hochwertiger Metalle starkem Wandel unterwerfen wird, kann der 4D-Druck diese Tendenz verstärken, bietet aber zugleich bei früher Innovationsgestaltung hohes Potential hinsichtlich nachhaltiger Produkt- und Prozessinnovationen. In Österreich ließen sich über eine frühe Förderung der Technologien viele verschiedene Branchen und Akteure verbinden. Darüber hinaus bietet die Technologie sowohl Potentiale für etablierte Unternehmen (insbesondere für hochspezialisierte Maschinenbauer) als auch für die Start-Up Szene.

\section{Zitierte Quellen}

Glenn, J. C. und Florescu, E., 2015, 2015-16 State of the Future.

Kuang, X., Roach, D. J., Wu, J., Hamel, C. M., Ding, Z., Wang, T., Qi, H. J. (2019). Advances in 4D Printing: Materials and Applications. Advanced Functional Materials, 29(2), 1805290. doi:10.1002/adfm.201805290. 


\section{Cyborg: Gehirn-Computer-Schnittstellen}

Die „Verschmelzung” von Menschen mit Maschinen, wie sie in der Sciencefiction oft thematisiert wird, wird zwar noch auf lange Zeit Vision bleiben, aber die schrittweise Überschreitung der Grenze zwischen Mensch und Maschine wird häufiger und die technologischen Voraussetzungen werden immer besser. ${ }^{1}$ Begonnen hat es mit Cochlea-Implantaten und anderen künstlichen Organen bzw. Gliedmaßen und heute ist bereits so vieles möglich, dass einige Menschen bereits daran denken, auch ohne medizinische Notwendigkeit teilweise zum "Cyborg“ zu werden, um ihre Fähigkeiten über das normale menschliche Maß hinaus zu erweitern. Die Forschung an Schnittstellen zwischen Computern und dem menschlichen Hirn ist ebenfalls gerade dabei, über die Grundlagenforschung hinaus zu gehen und die Vision der Verbesserung kognitiver Funktionen zu verwirklichen, etwa durch neue "Sinne“ (etwa für Magnetfelder, Infrarotlicht oder Radiowellen). In näherer Zukunft könnten bereits Brainchips zur Serienreife gelangen, die eine einfache Kommunikation zwischen Gehirn und Computern bzw. Smartphones ermöglichen und so zunächst beispielsweise Querschnittsgelähmten bei der Prothesensteuerung und der Steuerung von elektronischen Geräten helfen sollen ${ }^{2}$. Auch wenn Neuroenhancement im engeren Sinne noch einige Zeit Utopie bleiben dürfte (ITA 2016), stellen sich schon heute spannende Fragen, wie die Gesellschaft mit solchen "Cyborgs" umgehen würde, siehe die von den Transhumanisten angestoßene Debatte (Kurzweil 2014). Auch der Transhumanismus an sich, also das Verbessern des Menschen mit technologischen Mitteln, wird kontrovers diskutiert ${ }^{3}$ (siehe CRISPR-Mensch).

So wird etwa in der Medizinethik diskutiert, ob nicht-kuratives Verbessern menschlicher Leistungen durch technische Implantate vertretbar wäre bzw. unter welchen Voraussetzungen. ${ }^{4}$ Es stellt sich weiters die Verteilungsfrage, wer Zugang zu solchen Verbesserungen bekommen wird und unter welchen Bedingungen. Für die einzelnen Anwendungen müssten jedenfalls prinzipielle Sicherheitsaspekte vorab geklärt werden, in erster Linie betreffend die Gesundheit des Cyborgs selbst, da es ja um das neuartige und komplexe Zusammenspiel von technischen mit natürlichen „Komponenten“ geht. Aber auch die Tatsache, dass Cyborgs übermenschliche Fähigkeiten haben würden, bedeutet etwas für das zukünftige Zusammenleben.

1 Techno Sapiens - Die Zukunft der Spezies Mensch. Film von Peppo Wagner - Erstausstrahlung in 3sat am 16.November 2016 pwfilm.at/dokumentation/techno-sapiens/.

2 Siehe die letzten Fortschritte und Ziele des US-Unternehmens Neuralink neuralink.com.

3 derstandard.at/story/2000117192353/transhumanismus-muss-der-menschoptimiert-werden.

4 pewinternet.org/essay/human-enhancement-the-scientific-and-ethicaldimensions-of-striving-for-perfection/. 
In Österreich gibt es einen international wettbewerbsfähigen Medizintechnik-Sektor ${ }^{5}$ sowie Forschung zum Thema Human-Computer-Interaction. ${ }^{6}$

\section{Zitierte Quellen}

ITA, 2016, Hirndoping - alte Träume, ernüchternde Realität, ITA-Dossier 24, November (Autor: Torgersen, H.), epub.oeaw.ac.at/ita/ita-dossiers/itadossier024.pdf.

Kurzweil, R., 2014, Menschheit 2.0. Die Singularität naht, 2. Aufl., Berlin: Lola Books

5 lifescienceaustria.at/life-science-in-austria/medical-devices/devices/.

6 Etwa am AIT (rund um M. Tscheligi) oder an der TU Wien (rund um G. Fitzpatrick). 


\section{Künstliche Organe - 3D-Biodruck}

Menschliche Organe im Labor zu züchten kennzeichnet ein Set an Forschungsansätzen, die aktuell erweitert und verfeinert werden. Nach Versuchen, Tierherzen zu züchten, wird das Tissue Engineering - die Konstruktion von Gewebe - als vielversprechender Weg angesehen. Weitreichende Visionen des Forschungsfeldes sind biologische Ersatzorgane aus dem 3D-Drucker. ${ }^{1}$ Während z. B. funktionsfähige Nieren oder Lungen aus dem Bio-Drucker spekulative Entwicklungen sind, rückt die Entwicklung von künstlichen Knorpeln, Knochen und Muskeln bereits näher (Matai, Kaur, Seyedsalehi, McClinton, \& Laurencin, 2020).

Die zukünftige Relevanz ist hoch: Auch wenn die Transplantation von Organen heute medizinisch fast Routine ist, so ist diese Form des Organersatz mit vielen Problemen verbunden: Die Medikamente zur Unterdrückung von Abstoßungsreaktionen beeinträchtigen die Lebensqualität umfassend; das zugrundeliegende Konzept des Hirntods der Spenderlnnen ist ethisch umstritten; es gibt einen steigenden Mangel an Organen, die transplantiert werden können; der Organhandel breitet sich als transnationale Kriminalität aus und schließlich wird der Organmangel durch den demografischen Wandel verstärkt. Steigende Lebenserwartung und demografischer Wandel werden das Problem des Mangels an Organspenden erhöhen. Künstliche Organe könnten diese Probleme umfassend lösen.

Allerdings besteht bei den 3D-Biodruck Organen ein hoher Grad an Unsicherheit in Bezug auf die Realisierungswahrscheinlichkeit und zugleich ist der Zeithorizont der Realisierung von biologischen Organen mit komplexen Funktionen schwer einzuschätzen. Da Österreich international bei den durchgeführten Organtransplantationen einen der Spitzenränge einnimmt, ist eine hohe Kompetenz vorhanden und somit sind gute Bedingungen für die Forschung gegeben.

\section{Zitierte Literatur}

Matai, I., Kaur, G., Seyedsalehi, A., McClinton, A., \& Laurencin, C. T. (2020). Progress in 3D bioprinting technology for tissue/organ regenerative engineering. Biomaterials, 226, 119536. doi: doi.org/10.1016/j.biomaterials.2019.119536.

1 Die künstlichen Organe der Zukunft sind dadurch charakterisiert, dass sie biologischer Herkunft und personalisiert sind. Synonyme sind daher „biological organs“, personalized organs“, siehe Glenn/Florescu (2015), früher auch: bioartifizielle Organe (dip21.bundestag.de/dip21/btd/14/031/1403144.pdf). 


\section{Funktionelle Nahrung aus dem Labor}

\section{Zusammenfassung}

Nahrungsmittel aus dem Labor bedienen verschiedene Ansprüche: So soll etwa Fleisch ohne Tiere wachsen, um die Fleischnachfrage umweltschonender zu befriedigen; Lebensmittel sollen mit genau dem Nährwert oder Arzneimittelgehalt produziert werden, der individuell gewünscht oder sogar auf Basis einer Genomanalyse empfohlen wurde. Gesundheitsfördernde, vorbeugende Wirkungen stehen dabei im Vordergrund, aber auch die gezielte Bekämpfung von Krankheiten ist denkbar. Visionen gehen bis zum 3D-Druck individualisierter Lebensmittel dort, wo sie verzehrt werden. Die Wirkung solcher angereicherten Nahrung bleibt allerdings teils ungewiss und auch schädliche Auswirkungen sind bei Einführung neuer, der menschlichen Verdauung unbekannter Substanzen denkbar. Funktionelle Lebensmittel sind ein stetig wachsender Markt; derzeit werben viele Lebensmittelhersteller mit gesundheitsfördernder Wirkung, auch wenn von Seiten der EU bereits 2006 regulierend eingegriffen wurde. So vielversprechend Innovationen im Nahrungsmittelbereich auch scheinen, stehen sie doch auch im Gegensatz zur zunehmenden Wertschätzung natürlicher Lebensmittel und deren gesundheitsfördernder Wirkung, die auf langer Erfahrung und Ernährungsgewohnheiten beruhen. Unbeachtet ist derzeit welche langfristigen Auswirkungen diese Entwicklungen auf Landwirtschaft und Ernährung sowie das Verhältnis von Mensch und Tier haben können.

\section{Überblick zum Thema}

Fortschritte in der Biotechnologie ermöglichen es, biologische Systeme, wie z. B. Bakterien, Tier- oder Pflanzenzellen so zu kultivieren, dass sie kommerziell wichtige organische Materialien und Moleküle herstellen. Diese kommen in der Arzneimittel-, Lebensmittel- und Getränkeindustrie sowie bei industriellen Anwendungen bereits zum Einsatz. Die Zellen wachsen dabei meist in Bioreaktoren - großen mit einer Nährlösung gefüllten Glas- oder Plastikbehältern - und können sowohl natürlichen Ursprungs sein als auch gentechnisch verändert oder synthetisch hergestellt sein. Konkrete Anwendungen sind zum Beispiel Laborfleisch oder auch Algen, die als Nahrungsergänzungsmittel kultiviert werden und eine ortsunabhängige Produktion ermöglichen. Zusätzlich können beliebige Inhaltsstoffe zugefügt oder angereichert werden. Auch herkömmlich wachsende Pflanzen werden gentechnisch verändert, um so eine Anreicherung von einer bereits vorhandenen oder auch komplett neuen Substanz zu erreichen. Solche sogenannten funktionellen Lebensmittel versprechen eine gesundheitsfördernde Wirkung. Insgesamt soll es so möglich werden, Nahrungsmittel auf individuelle Bedürfnisse besser zuzuschneiden. 
Vision individualisierte Lebensmittel aus dem 3D-Drucker

Laborfleisch
Eine Vision sind beispielsweise individualisierte Lebensmittel aus dem 3DDrucker, angepasst auf Ernährungsbedürfnisse, die sich aus dem eigenen DNA-Profil ergeben. Neben Nährstoffen könnten auch Arzneimittel in der gewünschten individuellen Dosis beigefügt werden, was Anwendungen in Pflege, Betreuung und Gerontologie möglich machen würde, aber auch Gefahrenpotentiale birgt (unsichtbare Arzneimittel z. B. als mögliche freiheitsbeschränkende Ruhigstellung).

Japan war 1991 das erste Land, das eine eigene Regulierung zur Anerkennung von funktionellen Lebensmitteln verabschiedete, genannt FOSHU Foods of specified health use - und eine Kennzeichnung einführte. Hier wird klar definiert, was ein Lebensmittel zu einem Funktionellen macht. Bisher finden sich mehr als 1.000 Produkte auf der Liste, darunter neben Knoblauch auch eine mit Ballaststoffen angereicherte Cola-Variante. Bei einer Erneuerung, die sich an der US-Gesetzgebung orientiert, wurde es 2015 Firmen gesetzlich ermöglicht, auf Basis eigener Evidenz mit gesundheitsfördernder (funktioneller) Wirkung zu werben. ${ }^{1}$ Beim ursprünglichen FOSHU-Gesetz waren noch klinische Studien nötig und die gesundheitsfördernde Wirkung auf 14 Teilgebiete begrenzt (Iwatani \& Yamamoto 2019). Der Markt wächst rasant, KonsumentInnenschutz scheint in Japan eine untergeordnete Rolle zu spielen. In der EU müssen hingegen gesundheitsbezogene Aussagen seit 2006 in einer zentralen Datenbank registriert werden ${ }^{2}$ und werden nur erlaubt, wenn solide wissenschaftliche Evidenz zugrunde liegt (Dominguez-Diaz et al. 2020). So vielversprechend Innovationen im Nahrungsmittelbereich auch scheinen, stehen sie doch auch im Gegensatz zur Debatte um natürliche Lebensmittel und deren gesundheitsfördernde Wirkung (Ozen et al. 2012), die auf langer Erfahrung und Ernährungsgewohnheiten beruhen.

Am medienwirksamsten wird derzeit Laborfleisch diskutiert, das vor allem als Alternative zur herkömmlichen Fleischproduktion gesehen wird. Die Nachfrage nach Fleisch steigt weltweit und die Produktion von Fleisch verbraucht ein Vielfaches an Ressourcen wie Land, Wasser und Energie, die für die Erzeugung pflanzliche Nahrung benötigt werden. Die Futterpflanzenproduktion nimmt dabei einen immer größeren Teil der begrenzten Ackerfläche ein. Studien zeigen, dass die derzeitige Nachfrage nach Fleisch nicht nachhaltig bedient werden kann (Böhm 2016). Massentierhaltung hat eklatante negative Auswirkungen auf die Umwelt, wie der beschriebene Ressourcenverbrauch oder Überdüngung der Böden mit Abfallprodukten und die damit einhergehende hohe Phosphatbelastung des Grundwassers. Zusätzlich zieht der hohe Einsatz von Antibiotika in der derzeitigen industriellen Fleischproduktion auch ehebliche Gefahren für menschliche Gesundheit nach sich, etwa die starke Vermehrung antibiotikaresistenter Keime.

\footnotetext{
1 mhlw.go.jp/english/topics/foodsafety/fhc/02.html.

2 ec.europa.eu/food/safety/labelling_nutrition/claims/register/public/ ?event=register.home.
} 
Vor diesem Hintergrund arbeiten Forscherlnnen und Unternehmen daran, Fleisch künstlich im Labor zu produzieren und so die Massenhaltung von Rindern, Schweinen und anderen Nutztieren zumindest teilweise zu ersetzen. Dabei wachsen tierische Muskelstammzellen in einem Nährmedium in einem Bioreaktor. 2013 stellten ForscherInnen der Universität Maastricht den ersten auf diesem Weg erzeugten Burger vor. Das US-Unternehmen Memphis Meats erzeugt bereits künstliche Fleischbällchen und Geflügel ${ }^{3}$ und wird nach eigenen Angaben in fünf Jahren Marktreife erlangen. Ein israelisches Startup arbeitet an künstlichem Hühnerfleisch. ${ }^{4}$ Trotz viel Forschung wurden in den letzten Jahren keine eklatanten Fortschritte auf dem Gebiet gemacht (Chriki \& Hocquette 2020).

Theoretisch kann Zellkultivierung im Vergleich zu Nutztierhaltung effizienter Protein produzieren und Nährwerte können nach Belieben eingestellt werden. Derzeit sind aber noch Herausforderungen im Herstellungsprozess, bei Textur und Geschmack zu lösen, beispielsweise wird als Nährmedium oft fetales Blutserum von geschlachteten Kälbern verwendet, auch wenn einige Unternehmen angeben, bereits nicht-tierische Alternativen gefunden zu haben. Insgesamt konnte bisher gezeigt werden, dass zwar weniger Land und Wasser für die Herstellung von Laborfleisch verbraucht wird, der Energiebedarf aber höher ist. Weitgehend unbeachtet ist bisher, wie KonsumentInnen auf Laborfleisch reagieren könnten und welche langfristigen Auswirkungen es auf Landwirtschaft und Ernährung sowie auf das Verhältnis von Mensch und Tier haben kann (Böhm 2016).

\section{Relevanz des Themas für das Parlament und für Österreich}

Funktionelle Lebensmittel sind ein wachsender Markt und biotechnologische Innovationen machen ständig neue Produkte möglich; mit einer gesundheitsfördernden oder krankheitsbekämpfenden Wirkung werben zu können, ist für viele Hersteller äußerst attraktiv. Fragen, die das Parlament hier stellen kann, sind: Ist die bestehende EU-Regulierung im Sinne der KonsumentInnen stark genug? Werden unbekannte Wirkungen von synthetischen Nahrungsmitteln auf den Menschen ausreichend abgeklärt? Ist ein Kennzeichnungssystem für Produkte mit wissenschaftlich erwiesener gesundheitsfördernder Wirkung denkbar? Welche langfristigen Auswirkungen könnten Lebensmittel aus dem Labor auf die herkömmliche Landwirtschaft haben und wie könnten diese aktiv gesteuert werden? Soll Essen nur als Instrument zur Gesundheit reguliert werden, oder gibt es andere wichtige kulturelle Aspekte, die berücksichtig werden müssen? Welche positiven und negativen Folgen könnte eine individualisierte Ernährung auf Basis des persönlichen DNA-Profils auf den einzelnen und die Gesellschaft haben? Gibt es hier Steuerungsbedarf? Fleischproduktion ist ein sehr großer Markt und Laborfleisch könnte sich auf diesem als „ethisch vertretbares Fleisch" etablieren. Wie wird es rechtlich behandelt werden, soll es mit herkömmlichem Fleisch gleichgestellt sein?

\footnotetext{
3 memphismeats.com

4 supermeat.com.
}

wachsender Markt

Regulierung?

langfristige

Auswirkungen?

kulturelle Aspekte 


\section{Vorschlag weiteres Vorgehen}

Die oben gestellten Fragen könnten überblicksartig in einer Kurzstudie mit begrenzter Gültigkeit geklärt werden. Für eine tiefgreifende Klärung dieser und auch weiterer relevanter, aber bisher noch nicht gestellter Fragen, die auch längerfristige Planungssicherheit bieten würde, wäre allerdings eine Langstudie nötig. Diese müsste neben wissenschaftlicher Literaturrecherche vor allem auch alle relevanten Stakeholder-Gruppen einbinden, und zusätzlich auch BürgerInnen beteiligen.

\section{Zentrale weiterführende Quellen}

Böhm, I., 2016, Visionen von In-vitro-Fleisch - In-vitro-Fleisch als nachhaltige Lösung für die Probleme des Fleischkonsums? Technikfolgenabschätzung - Theorie und Praxis 25. Jg., Heft 1. tatup-journal.de/downloads/2016/tatup161_boeh16a.pdf.

Chriki S and Hocquette J-F (2020) The Myth of Cultured Meat: A Review. Front. Nutr. 7:7. doi: 10.3389/fnut.2020.00007.

Domínguez Díaz, L., Fernández-Ruiz, V., Cámara, M. (2020) An international regulatory review of food health-related claims in functional food products labeling, Journal of Functional Foods, Volume 68, ISSN 1756-4646, doi.org/10.1016/j.jff.2020.103896.

Iwatani, S., Yamamoto, N. (2019) Functional food products in Japan: A review, Food Science and Human Wellness, Volume 8, Issue 2, 96-101, ISSN 2213-4530, doi.org/10.1016/j.fshw.2019.03.011.

Ozen, A., Pons, A., Josep Tur, 2012, Worldwide consumption of functional foods: a systematic review. Nutrition Reviews, Volume 70, Issue 8, 1.8.2012, 472-481, doi.org/10.1111/j.1753-4887.2012.00492.x. 


\section{$\mathrm{KI}$ im Gesundheitswesen}

\section{Zusammenfassung}

Künstliche neuronale Netzwerke können gut darauf trainiert werden, Bilder zu vergleichen, um Muster und Abweichungen zu erkennen (siehe auch Thema „Deep Fakes“). Deshalb werden immer mehr KI-Systeme zur Analyse komplexer medizinischer Daten, wie MRT-Bilder oder EKGDaten entwickelt, die autonom Diagnosen mit teils sehr hoher Genauigkeit und vor allem Schnelligkeit erstellen. Ein weit verbreiteter Einsatz von KIbasierten intelligenten und autonomen Systemen in der Medizin kann das Risiko von Systemfehlern mit schweren Konsequenzen erhöhen und wirft komplexe ethische und gesellschaftliche Fragen auf. Künstliche Intelligenz $(\mathrm{KI})$ hat hier das Potential, professionelle Beziehungen, die Kommunikation mit PatientInnen, Wissenshierarchien, und den Arbeitsmarkt zu verändern. Im Folgenden zeigen zwei Beispiele Chancen und Risiken des Einsatzes von künstlicher Intelligenz im Gesundheitswesen auf: im hochsensiblen Bereich der Analyse medizinischer Daten und der darauf basierenden Diagnose sowie im Krankenhausmanagement.

\section{Überblick zum Thema}

KI-Diagnose-Systeme werden zurzeit vor allem in der Radiologie eingesetzt, können in vielen Medizinbereichen zur Anwendung kommen. Die einzige Voraussetzung sind genügend vergleichbare Daten zur Mustererkennung, wie etwa in der Blutanalyse oder der Überwachung von Vitaldaten. In der Radiologie werden KI-Anwendungen eingesetzt, die Röntgenoder MRT-Bilder eines Menschen innerhalb weniger Minuten mit Tausenden Aufnahmen anderer Menschen vergleichen und mit hoher Wahrscheinlichkeit richtige Diagnosen stellen können. Die Maschinen arbeiten zwar nicht fehlerfrei, können aber Entscheidungen mit höherer Konsistenz als Menschen treffen. KI wirkt hier nicht nur bei der Bildinterpretation mit, sondern auch bei Berichterstellung, Ergebniskommunikation und Abrechnung (Geis et al. 2019). Insgesamt erreichen KI-Diagnosesysteme bei der Analyse medizinischen Bildmaterials momentan etwa die gleiche Genauigkeit wie Fachpersonal (Liu et al. 2019), sie sind aber viel schneller.

Die automatisierte Analyse medizinischer Daten kann helfen, mit hoher Wahrscheinlichkeit Krankheiten vorherzusagen, bevor Symptome auftreten. So kann eine KI-Diagnose z.B. Alzheimer Jahre vor Symptombeginn anhand von MRT-Bildern erkennen was eine sehr frühe und damit effektive Behandlung erlaubt (Bhagwat et al. 2018). Lernende Algorithmen können ebenfalls Brustkrebs mit höherer Genauigkeit als ExpertInnen in Mammographien erkennen und ermöglichen so eine frühestmögliche Behandlung (McKinney et al. 2020). Leukämie konnte mit Hilfe von KI anhand der Aktivierung bestimmter Gene mit hoher Genauigkeit und zum Bruchteil der Kosten bisheriger Verfahren diagnostiziert werden (WarnatHerresthal et al. 2020). Auch Darmkrebs kann durch die automatisierte
Chancen: $K I$ verbessert

Diagnose \&

Krankenhausverwaltung

Risiko von

Systemfehlern und

schweren Folgen

KI-Diagnose:

effizienter als Ärztınnen

Frühzeitige Erkennung mit KI: Alzheimer, Krebs und Sepsis 
KI-Diagnose von Herzinsuffizienz

Entscheidungen treffen ÄrztInnen, auch in Zukunft

Wann KI vertrauen - und wann nicht?

Fehlender ethischer Rahmen für KI-Diagnose

Ethisch bedenkliche Geschäftsmodelle
Blutprobenanalyse von Tumor-DNA früher und nicht-invasiv erkannt werden (Phallen et al. 2017). Kl-Algorithmen können auch helfen, Sepsis (Blutvergiftung) bei IntensivpatientInnen mehrere Stunden vor klassischem Symptombeginn zu erkennen, indem Vitaldaten in Echtzeit überwacht und -analysiert werden (Garnacho-Montero/Martín-Loeches 2020).

Eine von Forscherlnnen trainierte KI schaffte es kürzlich sogar, mit 100\%Genauigkeit kongestive Herzinsuffizienz, also eine verminderte Pumpfähigkeit des Herzens, in EKG-Daten anhand weniger Herzschläge zu diagnostizieren. Ihr Einsatz könnte z.B. die oft benötigten Langzeit EKGs auf wenige Minuten verkürzen (Porumb et al. 2020).

Viele Kl-getriebene Diagnosesysteme machen Vorschläge mit Wahrscheinlichkeiten für Diagnosen und auch Differenzialdiagnosen, also abweichende mögliche Diagnosen, aber die Letztentscheidung treffen ÄrztInnen. Das wird auch in Zukunft wohl gesetzlich gesehen eher so bleiben, aber auch de facto? Inwieweit kann medizinisches Personal die Diagnosen der Algorithmen überhaupt nachvollziehen und innen damit vertrauen? Als Verantwortliche für Entscheidungen müssten sie dies aber können. Ein Problem, nachdem die meisten KI-Diagnose-Systeme BlackBoxes bleiben, wenn ÄrztInnen keine eigene Ausbildung zu ihrem Verständnis mitbringen.

Die Genauigkeit eines Ki-Diagnose-Systems lässt sich zwar in Studien bestimmen, aber die Frage bleibt, wann ÄrztInnen der KI-Diagnose in ihrer Entscheidung folgen und wann nicht. Dieses Problem könnte relevant werden, wenn etwa MedizinerInnen z.B. aus Angst vor Klagen den statistisch genaueren KI-Diagnosen folgen, auch wenn sie im Einzelfall eventuell eine andere Einschätzung haben. Auch sollte geklärt werden wie junge ÄrztInnen genug eigene Erfahrungen aufbauen können ohne sich auf die Systeme zu verlassen.

$\mathrm{KI}$ hat großes Potenzial, um die Effizienz und Genauigkeit in der gesamten Radiologie zu erhöhen, aber ihr Einsatz birgt auch inhärente Fallstricke und Verzerrungen. Mehrere europäische und nordamerikanische Radiologie-Vereinigungen haben kürzlich ein gemeinsames Statement veröffentlicht (Geis et al. 2019). Sie weisen auf die Notwendigkeit der Entwicklung eines ethischen Rahmens für $\mathrm{KI}$ in der Radiologie hin und zeigen auf, welche veränderten Anforderungen und offene Fragen aufgrund des vermehrten klinischen Einsatzes entstehen. Hierzu zählen vor allem die notwendige Risikobewertung jedes Systems, die Anpassung der Ausbildung von ÄrztInnen, das ständige Testen von Trainingsdaten sowie ein laufendes Monitoring der Effekte.

ÄrztInnen sind einerseits ethisch dazu verpflichtet, Daten, die sie sammeln, zum Besten von PatientInnen und des Gemeinwohls zu nutzen und ihr Fach zu verbessern. Andererseits dürfen sie Daten nicht so verwenden, dass PatientInnen geschädigt oder diskriminiert werden könnten. Da z.B. die Qualität radiologischer Daten stetig zunimmt, steigen auch deren Wert und damit Interesse und Druck, diese kommerziell zu verwerten. Ethische und insbesondere auch ethisch fragwürdige Geschäftsmodelle, wie z.B. 
die algorithmische Auswertung von Radiologiedaten zu rein finanziellen Zwecken ohne PatientInnen oder der Allgemeinheit zu helfen, entwickeln sich derzeit rascher als deren Bewertung erfolgen kann (Geis et al. 2019). Auch werden durch die Wertsteigerung medizinischer Daten Krankenhäuser vermehrt zu Angriffszielen von Hackern (Datengetriebene Medizin).

\section{$\mathrm{KI}$ im Krankenhausmanagement}

KI-basierte Systeme halten auch Einzug in die digitale Infrastruktur des Krankenhausmanagements. Diese können beispielsweise den Patientlnnenfluss über automatisierte Terminvergaben, Raum- und Ressourcenzuweisungen effizienter steuern. Chatbots sollen PatientInnen die gängigsten Fragen zu den nächsten Behandlungsschritten schnell beantworten. Belegte Effizienzsteigerung in speziellen Bereichen steht hier neben den (unbelegten) hohen, generellen Effizienzversprechen.

Aufgrund des Kostendrucks verwenden bereits viele US-Krankenhäuser und Krankenversicherungen Algorithmen, um Investitionen vorausschauend zu steuern. Kürzlich zeigte eine Studie, wie bestehende gesellschaftliche Ungleichheit über unausgewogene Trainingsdaten die Entscheidungen einer der größten kommerziellen Krankenhausmanagementsoftware beeinflusst, und damit ohnehin benachteiligte Personengruppen weiter benachteiligt (Obermeyer et al. 2019).

Der Algorithmus verwendet bereits entstandene Kosten eines Menschen im Gesundheitssystem als Proxy für gesundheitliches Risiko: je mehr Kosten in der Vergangenheit, desto höher das Gesundheitsrisiko in der Zukunft. JedeR Patient/n bekommt damit einen Risikowert zugeschrieben, der dann in Folge über die Zuweisung von Behandlungen mitentscheidet. Die im Gesundheitssystem entstandenen Kosten bilden in den USA aber strukturellen Rassismus ab: durch ungleichen Zugang zu Gesundheitsleistungen verursachen weiße PatentInnen statistisch mehr Kosten als afro-amerikanische. Zweitere sind damit bei gleichem Risikowert in schlechterem Gesundheitszustand, bekommen also statistisch gesehen eher später in ihrem Krankheitsverlauf benötigte Behandlungen zugewiesen.

\section{Relevanz des Themas für das Parlament und für Österreich}

KI-Einsatz in medizinischer Informatik und Technik wirft mehrere gesellschaftliche, ethische und politische Problemfelder auf, die z.T. auch beim generellen Einsatz von $\mathrm{KI}$ in anderen Anwendungsgebieten diskutiert werden (siehe auch Thema „KI-Risiken“). Da im Gesundheitsbereich aber hochsensible Daten verarbeitet werden und Entscheidungen hier gravierende Auswirkungen auf das Leben haben, ist besondere Vorsicht geboten. Bestehende Gesetzgebung muss angepasst werden, um einen adäquaten Umgang mit neuen technischen Entwicklungen, wie der KI-Diagnose zu gewährleisten (siehe auch: Datengetriebene Medizin). Datensicherheit und Schutz der Privatsphäre von PatientInnen steht im Spannungsfeld zwischen schneller und bestmöglicher Diagnose und Behandlung einerseits und freiem Datenzugang und -vernetzung für die Grundlagenforschung andererseits. Außerdem führen verzerrte $\mathrm{KI}$-Trainingsdaten-
Effiziente

Ressourcenverteilung und Chatbots

Algorithmischer

Risikowert bestimmt

Behandlungsleistungen

Problem Bias:

Verstärkung

existierender

Ungleichheiten

Hochsensible Daten und potentiell gravierende Folgen 
sätze immer wieder zu Fehlern und Diskriminierung bestimmter Gruppen (Bias). Wie können diese Fehler frühzeitig erkannt und verhindert werden? Wie sicher sind dann solche Systeme? Wie können Anwenderlnnen, für die derartige Systeme Black-Boxes sind, vertrauen oder Verantwortung für KI-Analysen übernehmen?

Außerdem ist zu hinterfragen, wer letztlich von Effizienzsteigerungen profitiert: Werden freie Ressourcen in neue Stellen und Geräte investiert? Haben ÄrztInnen mehr Zeit für ihre PatientInnen? Oder wird die Profitabilität von (privatisierten) Gesundheitseinrichtungen gesteigert?

\section{Vorschlag weiteres Vorgehen}

Eine explorative FTA-Studie kann die hier angerissenen Problemfelder eingehend betrachten, um konkrete Handlungsoptionen für Gesundheitspolitik und -management sowie Technikentwicklung abzuleiten. Im Zuge der Studie ist eine umfassende Stakeholderbeteiligung anzuraten, da die offenen Fragen zum Einsatz von $\mathrm{KI}$ in der Diagnose und Verwaltung ein Zusammenführen verschiedenen Wissens- und Erfahrungsgebiete erfordern.

\section{Zitierte Literatur}

Bhagwat, N. et al., 2018, Modeling and prediction of clinical symptom trajectories in Alzheimer's disease using longitudinal data, PLOS Computational Biology 14(9), e1006376 doi.org/10.1371/journal.pcbi.1006376.

Garnacho-Montero, J. und Martín-Loeches, I., 2020, Clinical management of sepsis can be improved by artificial intelligence: no, Intensive Care Medicine 46(2), 378-380 doi.org/10.1007/s00134-020-05947-1.

Geis, J. R. et al., 2019, Ethics of Artificial Intelligence in Radiology: Summary of the Joint European and North American Multisociety Statement, Radiology 293(2), 436-440 ncbi.nlm.nih.gov/pubmed/31573399.

Liu, X. et al. , 2019, A comparison of deep learning performance against health-care professionals in detecting diseases from medical imaging: a systematic review and meta-analysis, The Lancet Digital Health 1(6), e271-e297 doi.org/10.1016/S2589-7500(19)30123-2.

McKinney, S. M. et al., 2020, International evaluation of an Al system for breast cancer screening, Nature 577(7788), 89-94.

Obermeyer, Z. et al., 2019, Dissecting racial bias in an algorithm used to manage the health of populations, Science 366(6464), 447 science.sciencemag.org/content/366/6464/447.abstract.

Phallen, J. et al., 2017, Direct detection of early-stage cancers using circulating tumor DNA, Science Translational Medicine 9(403), eaan2415 stm.sciencemag.org/content/9/403/eaan2415.abstract.

Porumb, M., et al., 2020, A convolutional neural network approach to detect congestive heart failure, Biomedical Signal Processing and Control 55, 101597 sciencedirect.com/science/article/pii/S1746809419301776.

Warnat-Herresthal, S. et al., 2020, Scalable Prediction of Acute Myeloid Leukemia Using High-Dimensional Machine Learning and Blood Transcriptomics, iScience 23(1) doi.org/10.1016/j.isci.2019.100780. 


\section{Epigenetische Therapieansätze}

Damit PatientInnen maßgeschneiderte und auf ihre individuellen Gegebenheiten angepasste Therapien erhalten, rücken bei der Erforschung der molekularen Ursachen von Erkrankungen (siehe dazu auch die Themen "Datengetriebene Medizin“ und „Personalisierte Genomsequenzierung“) neben genetischen auch zunehmend epigenetische Faktoren ins Blickfeld der Medizin. Die Epigenetik (altgr. epi ,dazu', darüber und Genetik) untersucht den Einfluss von Umweltfaktoren (z.B. Ernährung, Lebensstil, Stress, Traumata, Umweltschadstoffe) auf die Zelleigenschaften und den Aktivitätszustand von Genen (,An- und Abschalten“ von Genen). Indem sich bestimmte chemische Moleküle (z.B. Methylgruppen) umweltbedingt an die DNA oder die DNA-Bindungseiweiße anhaften, werden die betroffenen DNA-Bereiche auf molekularer Ebene wie mit einer „roten Flagge" markiert. Solche epigenetischen Markierungen können die Funktionsweise von Genen auf den betroffenen DNA-Abschnitten beeinflussen, indem sie z.B. dazu führen, dass auf Teile der genetischen Informationen nicht mehr zugegriffen werden kann und dadurch bestimmte Zellprozesse nicht mehr ablaufen können. Fehlerhafte Markierungen können sich negativ auf die Gesundheit des Menschen auswirken und das individuelle Risiko für bestimmte - oft komplexe und chronische - Krankheiten erhöhen (z.B. Krebs, Herz-Kreislauf- und Stoffwechselerkrankungen, Depression). Obwohl epigenetische Markierungen nur chemische Anhaftungen an das eigentliche Erbgut darstellen und die DNA selbst nicht verändern, können sie trotzdem an nachfolgende Generationen vererbt werden.

Die Tatsache, dass epigenetische Markierungen unter bestimmten Umständen reversibel sind, ${ }^{1}$ d.h. auch wieder entfernt werden können, macht sich die Medizin bei der Erforschung neuer Medikamente und personalisierter Therapiemöglichkeiten für PatientInnen zunutze. Bisher sind jene Wirkstoffe am besten untersucht, die in epigenetische Prozesse bei der Krebsentstehung eingreifen (Ganesan et al. 2019). In Österreich sind bereits erste epigenetisch wirksame Medikamente zur Behandlung von Krebserkrankungen zugelassen (seit 2008 der Wirkstoff Azacitidin und seit 2012 der Wirkstoff Decitabin zur Behandlung akuter myeloischer Leukämie $(A M L))^{2}$. Einerseits ist Epigenetik ein vielversprechender Forschungsbereich für zukünftige, personalisierte Therapieansätze auf chemischer, psychischer und verhaltensorientierter Ebene. Vor allem eine daraus resultierende, bessere Kenntnis des molekular-biologischen $\mathrm{Zu}-$ sammenhangs zwischen individuellem Verhalten und chronischen Krankheiten könnte langfristig dazu beitragen, durch gezielte Präventivmaßnahmen zur Lebensstiländerung in der Bevölkerung, die Zahl chronisch

1 Psychiatrische Studien belegen, dass Psychotherapie epigenetische Veränderungen, die durch posttraumatische Belastungsstörungen verursachten wurden, rückgängig machen kann (Vinkers et al. 2019).

2 medonline.at/innere-medizin/onkologie/digital/n/2020/10049339/onkologischeforschung-auf-der-ueberholspur/. 
kranker Menschen zu reduzieren (Hibler et al. 2019) und dadurch Kosten im Gesundheitssystem einzusparen. Andererseits stellt sich aus gesellschaftlicher Perspektive die Frage - v.a. aufgrund des wissenschaftlichen und medialen Hypes rund um das Thema (Deichmann 2016) - , wie sehr durch epigenetische Therapieansätze das persönliche Verhalten des Einzelnen medikalisiert wird, d.h. dass Lebenserfahrungen, die bisher außerhalb des medizinischen Geltungsbereichs gestanden sind, pharmazeutisch beeinflusst werden. Gleichzeitig ist der Trend zu beobachten, dass Pharmaunternehmen aufgrund der hohen Kosten bei der Medikamentenherstellung bevorzugt Medikamente entwickeln, die für weitverbreitete Erkrankungen vorgesehen sind, anstatt für seltene oder aufwendig zu behandelnde Krankheiten. Dies könnte dazu führen, dass in Zukunft auch bei der Medikamentenentwicklung auf Basis der Epigenetik der Fokus auf „profitable“ Krankheiten, wie z.B. Diabetes gelegt wird, und dadurch nur ausgewählte Bevölkerungsgruppen am medizinischen Fortschritt teilhaben.

Wünschenswert wäre daher eine breite, gesellschaftliche Diskussion und Reflexion von zukünftigen Möglichkeiten und Risiken sowie ethischen und sozialen Aspekten, die mit individuellen Therapieansätzen einhergehen könnten. Gerade unter der Annahme, dass epigenetische Muster auch vererbt werden können, hätte der eigene Lebensstil Auswirkungen auf die Nachkommen. Fragen zur Verantwortung des Einzelnen gegenüber der eigenen Gesundheit sowie der Gesundheit der Nachkommen wären daher ebenso zu adressieren, wie Fragen zur Verantwortung des Staates gegenüber sozio-ökonomisch benachteiligten Bevölkerungsgruppen, deren ungünstige Umwelt- und Lebensbedingungen negative und damit krankheitsfördernde epigenetische Veränderungen begünstigen.

\section{Zitierte Literatur}

Deichmann U. (2016) Epigenetics:The origins and evolution of a fashionable topic. Developmental Biology 416 (2016) 249-254.

Ganesan A. et al. (2019) The timeline of epigenetic drug discovery: from reality to dreams. Clinical Epigenetics 11:174.

Hibler E. et al. (2019). Impact of a diet and activity health promotion intervention on regional patterns of DNA methylation. Clinical Epigenetics 11, 133.

Vinkers C. H. et al. (2019) Successful treatment of post-traumatic stress disorder reverses DNA methylation marks. Mol Psychiatry. 2019 Oct 23. doi: 10.1038/s41380-019-0549-3. 


\section{Xenobots: lebendige Roboter?}

Xenobots heißen die lebendigen, computer-designten Organismen, die US-amerikanische Forschende mithilfe eines Supercomputers entwickelt haben (Kriegman et al. 2020). ${ }^{1} \mathrm{lhr}$ Namensgeber und Zelllieferant ist Xenopus laevis (lat.), der Afrikanische Krallenfrosch oder Apothekerfrosch. Die Vorsilbe „xeno" (abgeleitet von griechisch xénos, was so viel bedeutet wie "Gast", „Fremder") verweist darauf, dass die zu einem Organismus zusammengefügten Zellen andere Funktionen als die von ihrem ursprünglichen genetischen Bauplan vorgesehenen erfüllen können. Von der Synthetischen Biologie (Synbio) vorangetrieben (siehe Thema Künstliches Leben), sind Xenobots ein erstes Beispiel für eine künstlich geschaffene Lebensform mit speziellen Eigenschaften. Sie bestehen aus ausschließlich lebenden, ${ }^{2}$ tierischen Haut- und (Herz-)Muskelzellen, die den Organismen durch ihr kontinuierliches Pulsieren eine eingeschränkte Fortbewegung ${ }^{3}$ ermöglichen. Darüber hinaus können die lebenden Maschinen schwimmen, kleine Lasten transportieren, in Gruppen zusammenarbeiten und sich selbst heilen. Wie ist es möglich, solche künstlichen Organismen zu entwickeln? Dazu entwirft ein Computer in vielzähligen Simulationen anhand eines „Evolutionsalgorithmus", d.h. mithilfe von Methoden der künstlichen Intelligenz, unterschiedliche Baupläne, aus denen geeignete Designs ausgewählt werden.

Welchen Nutzen versprechen sich die Forschenden von Xenobots? Die ersten Studien zu den lebenden Robotern dienen vordergründig dazu, die Machbarkeit dieser neuen Technologie aufzuzeigen und die Erforschung der „Mechanismen des Lebens“4 voranzutreiben. Die Zukunftserwartungen in Hinblick auf den gesellschaftlichen Nutzen gehen in zwei Richtungen, abhängig vom eingesetzten Zellmaterial. Xenobots, die aus Tierzellen gebaut werden, versprechen vielseitiges Anwendungspotenzial, bspw. beim Aufspüren radioaktiver bzw. toxischer Kontamination in verseuchten Gegenden oder bei der Reinigung der Meere von Mikroplastik. Werden die Mini-Organismen aus menschlichen Zellen hergestellt, könnten diese vor allem für die biomedizinische Forschung von hoher Relevanz sein. Dort sollen Xenobots, die aus körpereigenen Zellen einer z.B. an Krebs erkrankten Person entwickelt werden, in Folge von der betroffenen Person geschluckt werden und dann eigenständig den Weg zum Krebstumor

1 Gefördert wurde das Forschungsunterfangen durch das Programm „Lifelong Learning Machines (L2M)“ der DARPA, siehe darpa.mil/program/lifelonglearning-machines.

2 Die derzeitige Lebensdauer der Xenobots reicht bis zu sieben Tagen; mithilfe von nährstoffreichen Substraten kann ihre Lebensdauer auf mehrere Wochen ausgedehnt werden.

3 Das Video zeigt, wie sich ein Xenobot fortbewegt: youtu.be/WhFWNoBiKUc.

4 Erklärtes Ziel ist die Erforschung von biologischen Strukturen, um Kontrolle über Wachstum und Form von Organismen zu gewinnen, die bei der Lösung von Problemen, wie Geburtsfehler, Krebs, oder altersbedingten Krankheiten, helfen könnten. 
finden, um dort gezielt Medikamente abgeben zu können. Ähnlich wäre der Vorgang, um z.B. verkalkte Arterien von innen heraus von Plaque zu befreien. Ein Vorteil dieser Zukunftsanwendung wird dabei in der besseren Immunverträglichkeit der von außen eingebrachten Organismen gesehen, da sie aus körpereigenem Material entwickelt werden könnten. ${ }^{5}$

Obwohl diese Anwendungsszenarien noch in weiter Ferne liegen, stellen sich in Anbetracht der gegenwärtigen Entwicklung weitreichende ethische und soziale Fragen. Allen voran die zentrale Frage: Was sind Xenobots überhaupt und welchen Status hätten sie in unserer Gesellschaft? Erfüllen sie ausreichende Anforderungen, um als Lebewesen klassifiziert zu werden? Und ab wann sollten wir die künstlich erzeugten, lebendigen Organismen als Lebensformen mit Eigeninteressen ansehen und schützen? ${ }^{6}$ Die Forschung an den künstlichen, lebenden Maschinen erfolgt zu einem großen Teil öffentlich und unter Einbeziehung rechtlicher Expertise; auch der Quellcode, der für die Entwicklung der computer-designten Wesen eingesetzt wurde, ist kostenlos und öffentlich zugänglich. ${ }^{7}$ Trotzdem bleibt die Frage offen, wie das Bewusstsein der Gesellschaft für diese komplexe und noch wenig ausgereifte Entwicklung und ihre möglichen Konsequenzen geschärft werden kann. Ein breit angelegter ForesightProzess, der auf die Diskussion und Reflexion von Xenobots und ähnlichen Entwicklungen ${ }^{8}$ in der Gesellschaft abzielt, könnte die Grundlage für erwünschte, zukünftige Forschungs- und Entwicklungspfade und für notwendige und nachhaltige, politische Rahmenbedingungen liefern.

\section{Zitierte Literatur}

Kriegman, S., Blackiston, D., Levin, M. und Bongard, J., 2020, A scalable pipeline for designing reconfigurable organisms, Proceedings of the National Academy of Sciences 117(4), 1853.

Lavazza, A. und Massimini, M., 2018, Cerebral organoids: ethical issues and consciousness assessment, Journal of Medical Ethics 44(9), 606.

5 bioethics.net/2020/02/living-robots-ethical-questions-about-xenobots/.

6 Möglichkeiten zur Weiterentwicklung der Xenobots mit Blutgefäßen, Nervensystemen, Sinneszellen und Augen werden von den Forschenden bereits angedacht, siehe theguardian.com/science/2020/jan/13/scientists-use-stem-cellsfrom-frogs-to-build-first-living-robots.

7 github.com/skriegman/reconfigurable_organisms.

8 In engem Zusammenhang mit der ethisch-sozialen Debatte um Xenobots stehen Organoide (organähnliche Mikrostrukturen, die aus menschlichen Zellen gezüchtet werden und z.B. für Entwicklung von individuell angepassten Medikamenten genutzt werden). Zu ethischen Aspekten von Organoiden siehe Lavazza/Massimini (2018). 


\section{Datengetriebene Medizin - Zwischen Personalisierung und gläsernen PatientInnen?}

\section{Zusammenfassung}

Die datengetriebene Medizin - die umfangreiche Nutzung von Big Data in der Medizin und der Pharmaindustrie - verspricht sowohl bessere Diagnosen und schnellere Herstellung neuer Medikamente als auch passgenaue Therapien und maßgeschneiderte, personalisierte Behandlung. Möglich wird sie mit der Etablierung von elektronischen Gesundheitsakten, kostengünstiger Genomsequenzierung, Biobanken, molekularer Bildgebung, PatientInnenportalen und gesundheitsrelevanten Sensoren in Smartphones. Damit wächst aber auch der "digitale Fußabdruck“ von PatientInnen rasant. Dazu kommen die digitalen Spuren aller Menschen, die im öffentlichen Gesundheitssystem und auf den Märkten für Gesundheitsdienstleistungen ihre Daten zu Präventions- und Forschungszwecken Dritten überlassen. Weitere relevante Daten entstehen bei der onlineSuche nach gesundheitsrelevanten Informationen, beim Kauf gesundheitsbezogener Produkte und Dienstleistungen und schließlich in der Kombination: wenn direkt gesundheitsrelevante Daten mit Daten gekoppelt werden, die den Lebensstil identifizierbar machen, wie Daten aus sozialen Netzwerken und Daten der Online-Nutzung. Wie eine datengetriebene Medizin langfristig einen hohen Nutzen generieren kann, ohne dass Gesundheitsdaten zur Diskriminierung (zum Beispiel durch Versicherungen und ArbeitgeberInnen) genutzt werden, ist bisher nicht absehbar.

\section{Überblick zum Thema}

Mit dem Zugang zu enormen Mengen an heterogenen Daten (Big Data) und dem Zuwachs an Rechenleistung und ihren Verarbeitungsmöglichkeiten (Künstliche Intelligenz) verändert sich die Medizin und erschließt beispiellose Möglichkeiten für eine datengetriebene medizinische Forschung, die zu kostengünstigeren und personalisierten Behandlungen führen könnte. Damit könnte auch der beginnende Paradigmenwechsel in der Medizin von kurativen Maßnahmen hin zu prophylaktischen, präventiven Gesundheitsservices unterstützt werden. Die Versprechen der datengetriebenen Medizin sind hoch, denn ihre Verfechterlnnen versprechen nicht weniger als proaktive, prädiktive, präventive, partizipative und patientenorientierte Ansätze in der Medizin der Zukunft (Shah/Tenenbaum 2012). Diese Form der datengetriebenen Medizinforschung geht über die Kapazitäten nationalstaatlicher Forschung hinaus und ist nicht selten von Konzernen getrieben, wie z.B. von der führenden US-amerikanischen Genomsequenzierungs-Firma 23andme, an der Alphabet/Google beteiligt ist. Welche Auswirkungen die Geschäftsmodelle der führenden Unternehmen auf die zukünftige medizinische Forschung haben und worin die Treiber und Barrieren hinsichtlich der Potentiale bestehen, ist bisher unklar. digitaler Fußabdruck von PatientInnen wächst

Paradigmenwechsel in der Medizin von kurativen Maßnahmen zu prophylaktischen, präventiven Gesundheitsservices 


\author{
Verbindung von \\ Datensätzen \\ unterschiedlicher \\ Herkunft und Struktur
}

Krankenhäuser werden Angriffsziele von Hackern

Quantified-SelfBewegung
Die rasante Entwicklung der datengetriebenen Medizin besteht insbesondere darin, Datensätze unterschiedlicher Herkunft und unterschiedlicher Struktur zu verbinden: So werden DNA-Repositorien mit elektronischen Gesundheitsakten gekoppelt, um die Genomforschung voranzutreiben (McCarty et al. 2011), und elektronische Gesundheitsakten werden mit Biomarker-Datenbanken gekoppelt, um anhand der Biomarker Krankheitszusammenhänge zu identifizieren (Mosley et al. 2018).

Wenn neues medizinisches Wissen aus Millionen von Datensätzen generiert wird, das dann für die individuelle Diagnose und Therapie von spezifischen PatientInnen genutzt wird, stellen sich völlig neue Anforderungen an die Ausbildung und Praxis in den Gesundheitsberufen. Insbesondere die Interdisziplinarität an der Schnittstelle von Medizin, Datenwissenschaft und Informatik würde aus den Nischen der Spezialisierung in das Zentrum von Medizin und Gesundheitsforschung wandern. Diese datengetriebene Medizin erfordert interdisziplinäres Knowhow, eine veränderte Ausbildung wie auch Rahmenbedingungen für den Umgang mit Daten, d.h. Maßnahmen, die das Vertrauen in diese Form von Medizin und Medizinforschung langfristig sichern. Mit der steigenden Relevanz von Daten in der Medizin werden Krankenhäuser und Institutionen des öffentlichen Gesundheitswesens zugleich Angriffsziele von Hackern. ${ }^{1}$

Immer mehr Menschen nutzen aktiv eigene Gesundheitsdaten für die Optimierung ihrer Gesundheit oder für die eigene medizinische Behandlung, angefangen von personalisierten Gesundheitsprofilen aus den Daten von Fitness- und Aktivitäts-Trackern bis zur Genomsequenzierung. Nutzerlnnen, die über Sensoren und Smartphone ihre Daten mit anderen vergleichen, haben vor einigen Jahren in der sogenannten Quantified-SelfBewegung die elektronische Selbstvermessung zu einer Optimierung inrer Leistungsfähigkeit genutzt (Lupton 2013). KritikerInnen sehen darin das Ende von Freiheit und eine Gefahr für Datensicherheit. Heute ist die Nutzung von Geräten der Selbstvermessung weit verbreitet und überschneidet sich mit dem Gesundheitsmonitoring. Die Beteiligung von PatientInnen und potentiellen PatientInnen an der medizinischen Forschung verbindet Self-Tracking-Tools mit umfassenden Datenportalen, wobei unklar ist, ob den Einzelnen die Tragweite ihrer Datenweitergabe bewusst ist. Gerade bei Gesundheitsdienstleistungen ist das „Privacy Paradox" relevant: Zwar würden die meisten in einer Befragungssituation den Datenschutz hier als extrem wichtig ansehen, im Anwendungsfall dagegen werden Datenschutzerklärungen oft pauschal akzeptiert und sensible Daten preisgegeben. Denn der Nutzen einer gesundheitsrelevanten Dienstleistung erscheint im Gegensatz zu den abstrakten und langfristigen Folgen einer Datenweitergabe hoch.

12017 wurden Computersysteme mehrerer Krankenhäuser in Großbritannien im Zuge eines weltweiten Angriffs mit sogenannter Ransomware blockiert. Die Computer wurden über sogenannte Erpressungstrojaner verschlüsselt und dann wurde Lösegeld zur Entschlüsselung verlangt, siehe

derstandard.at/2000057473193/Loesegeld-gefordert-Hacker-greifen-englischeSpitaeler-an. 
Diese Ansätze einer „Crowdsourced Gesundheitsforschung“ (Swan 2012) verbindet die Tradition klinischer Studien mit strukturierten Selbstversuchen. Die Dynamik wird durch globale PatientInnenplattformen wie PatientsLikeMe ${ }^{2}$ vorangetrieben, die PatientInnen vernetzen, ihre Daten sammeln, diese Daten aber auch an Pharmaunternehmen verkaufen und im Auftrag von Pharmaunternehmen auch spezifische Daten sammeln. Neben den ethischen Fragen hinsichtlich einer solchen partizipativen Medizin, ${ }^{3}$ stellen sich Fragen langfristiger Folgen, die heute nur begrenzt absehbar sind. Einige der möglichen Risiken zeigen sich heute bereits in Ländern mit privaten Krankenversicherungen, die Preise nach Risiken gestalten. Deren flexible datengetriebene Tarife sind darauf ausgerichtet, Versicherte dazu zu bekommen, ihre Gesundheitsdaten über Geräte wie die Apple-Watch täglich an die Versicherungen zu übertragen. Vielfach sind diese Programme, die z.B. die Fitnessleistungen der Versicherten verfolgen, noch als Bonusprogramme konzipiert; können aber auch absehbar zu Diskriminierung führen. ${ }^{4}$

\section{Relevanz des Themas für das Parlament und für Österreich}

Wie es möglich ist, an der globalisierten, datengetriebenen Medizinforschung teilzunehmen und trotzdem langfristig ein hohes Datenschutzniveau aufrechtzuerhalten, ist weitgehend unerforscht, jedoch zentral für die Wirksamkeit und das Vertrauen in die Gesundheitssysteme der Zukunft. Da es sich um sensible, personenbezogene Daten handelt, muss die Erhebung und Verarbeitung unter Berücksichtigung der geltenden datenschutzrechtlichen Regelungen erfolgen. Das Spannungsverhältnis besteht darin, dass unklar ist, wie wirksam die heute geltenden Rechtsvorschriften sein können, wenn die Zusammenführung unterschiedlicher Daten technisch immer einfacher wird. In Bezug auf die heterogenen gesundheitsbezogenen Daten und ihre zukünftig immer leichtere Kombinierbarkeit ist keine absolute Datensicherheit möglich. Wesentlich ist allerdings, festzustellen, ob und welche Grade von Sicherheit heute möglich gemacht werden können, wenn zukünftige Entwicklungen gleichzeitig bereits antizipiert werden. Insbesondere die mögliche Re-Identifizierung der Daten durch zukünftige Künstliche-Intelligenz-Technologien und der Verlust von Anonymität tangieren die Privatsphäre sowie Eigentums- und Persönlichkeitsrechte und können zu Diskriminierungen führen.

\section{Vorschlag weiteres Vorgehen}

Aufgrund der Langfristigkeit der Fragen und der potentiellen Konfliktträchtigkeit wäre eine umfassende, auf Österreich fokussierende ForesightStudie mit partizipativen Elementen sinnvoll, die über die Analyse heutiger flexible datengetriebene Tarife bei Krankenversicherungen

Aufrechterhaltung

des hohen

Datenschutzniveaus

\footnotetext{
2 patientslikeme.com.

3 bundeskanzleramt.gv.at/dam/jcr:7df6165e-2e0f-45c8-86e660a3dd0ab4ec/MedizinInternet_Ansicht.pdf.

4 netzpolitik.org/2018/tracking-durch-die-versicherung-zu-risiken-undnebenwirkungen/.
}

Langfristigkeit und potentielle Konfliktträchtigkeit 
Technologien und ihrer Folgen hinaus zukünftige Entwicklungen umfassend antizipiert. Eine transdisziplinäre Studie würde das Wissen von ExpertInnen aus Medizin, Datenwissenschaften und KI-Forschung mit den Wissensbeständen und Interessen der verschiedenen Stakeholder (Gesundheitswesen, Pharmaindustrie, PatientInnengruppen) verbinden, um insbesondere die langfristigen Folgen von aktuellen Ansätzen zu analysieren und unterschiedliche Zukunftsoptionen für den Umgang mit Daten festzustellen. Ziel wäre es auch, wirksame Lösungsansätze für die globale Dimension der datengetriebenen Medizin zu erarbeiten.

\section{Zentrale weiterführende Quellen}

Lupton, D., 2013, Quantifying the body: monitoring and measuring health in the age of mHealth technologies, Critical Public Health23(4), 393-403.

McCarty, C. A., et al., 2011, The eMERGE Network: A consortium of biorepositories linked to electronic medical records data for conducting genomic studies, Bmc Medical Genomics 4.

Mosley, J. D., et al., 2018, A study paradigm integrating prospective epidemiologic cohorts and electronic health records to identify disease biomarkers, Nature Communications 9.

Shah, N. H. und Tenenbaum, J. D., 2012, The coming age of data-driven medicine: translational bioinformatics' next frontier, Journal of the American Medical Informatics Association19(E1), E2-E4.

Swan, M., 2012, Crowdsourced Health Research Studies: An Important Emerging Complement to Clinical Trials in the Public Health Research Ecosystem, Journal of Medical Internet Research14(2). 


\section{Zukunft der Mensch-Maschine-Interaktion: Haptische Holographie}

Haptische Holographie ist die Forschung und Technikentwicklung an der Schnittstelle digitaler Holographie und Haptik (Berührung, Tasten). ${ }^{1}$ Haptische Holographie umfasst verschiedene Arten von digitalen Hologrammen, die mittels unterschiedlicher Technologien (z.B. taktiles Feedback durch Vibration) haptisch erfahrbar gemacht werden sollen. Digitale Hologramme bezeichnen dabei im weiten Sinn digital gesteuerte Objekte, wie z.B. Roboter, 3D-Video- und Laserprojektionen, oder auch räumliche Illusionen, die durch Spiegelung von computergenerierten 3D-Bildern erzeugt werden. Was futuristisch anmutet, ist längst Realität: 2009 gelang es japanischen Forschern holographisch projizierte Regentropfen im freien Raum fühlbar zu machen (Hoshi et al. 2009). Dabei machte sich das Team ein Ultraschallphänomen - Schallstrahlungsdruck - zunutze, um haptisches Feedback mit einer 3D-Spiegelung von Regentropfen zu verbinden. Tatsächlich freischwebende 3D-Bilder können durch Laserplasmatechnologie (Nano-/Femtosekundenlaser) erzeugt werden (Ochiai et al. 2016). In Österreich erforschen unter anderem das Media Interaction Lab (MiL) der FH Oberösterreich, das Linz Center of Mechatronics $\mathrm{GmbH}$, das Institut für Softwaretechnik und Interaktive Systeme der TU Wien sowie das Center of Technology Experience des AIT, wie virtuelle Welten für taktile Wahrnehmung zugänglich gemacht werden können.

Für die Zukunft kann diese Schnittstellen-Technologie disruptiv wirken: Im Online-Handel würde haptische Holographie das virtuelle Erkunden von Produkten ermöglichen (z.B. Anprobieren von Kleidung), in der Produktentwicklung können Prototypen durch haptische Hologramme schneller, einfacher und günstiger zur Verfügung gestellt werden. ${ }^{2}$ Weitere relevante Anwendungen wären die Nutzung von haptischer Holographie in der Bildung und praktischen Ausbildung, z.B. zum Erlernen chirurgischer Verfahren (Page 2013) oder in der Kommunikation, z.B. 3D-Videotelefonie mit Berührung. Die fühlbare Interaktion mit digitalen Avataren ${ }^{3}$ würde die Mensch-Maschine-Interaktion in Richtung einer stärkeren ,Erfahrbarkeit' vorantreiben und neue Modi des Zusammenlebens („virtuelle LebensgefährtInnen“) und der Zusammenarbeit („Telearbeit 2.0“) ermöglichen. Für die Umwelt könnte die Nutzung virtueller Räume besondere Vorteile in Hinblick auf $\mathrm{CO}_{2}$-Reduktion und Eindämmung des Biodiversitätsverlusts haben. Haptische Hologramme könnten z.B. seltene Tiere in den dazugehörigen virtuellen Ökosystemen für viele Menschen digital zugänglich machen und dabei gleichzeitig sensible Lebensräume vor übermäßiger,

\footnotetext{
1 Beispielsweise forscht die Object-Based Media Group des MIT Media Labs zu haptischer Holografie, siehe media.mit.edu/groups/object-basedmedia/overview/.

2 derstandard.at/999809/Star-Wars-Hologramme-werden-Realitaet.

3 vrodo.de/forschung-aus-japan-bildtelefon-mit-beruehrung/.
} 
menschlicher Nutzung schützen. ${ }^{4}$ Virtuelle statt reale Urlaubsreisen ${ }^{5}$ könnten beträchtliche $\mathrm{CO}_{2}$-Einsparungen im Flugverkehr bringen; allerdings könnte eine attraktive virtuelle Reise auch die Nachfrage nach eben diesen steigern. Für Museen, Bildungseinrichtungen und den Kreativbereich (Design) ist die Anwendung haptischer Holographie attraktiv, z.B. um Bilder, Präparate, Installationen multisensorisch erlebbar zu machen (Vi et al. 2017).

Die theoretische Substitution der Realwelt durch "Holodeck-Technologie“ (ein Kunstbegriff aus der TV-Serie „Star Trek“) bietet Chancen für ökologische Nachhaltigkeit (Reduktion des Flugverkehrs, Vermeidung ressourcenintensiver Werbung und Produktion, Schonung sensibler Ökosysteme). ${ }^{6}$ Die Kombination von haptischer Holographie mit Ansätzen digitaler Verhaltenssteuerung im globalen Risikomanagement (World Economic Forum 2018) birgt zugleich ein hohes gesellschaftliches Manipulationspotential. Gerade in Anbetracht der Wirksamkeit taktilen Feedbacks sind die Chancen und Risiken dieser Zukunftstechnologie stark vom Anwendungskontext abhängig. Während haptische Hologramme in der Bildung, Produktion und Kommunikation hohes Potenzial in Hinsicht auf verbesserte Lernprozesse und interaktive Lernobjekte aufweisen, sind auch dystopische Anwendungsmöglichkeiten vorstellbar. Gerade in Bereichen wie Internetpornographie und Sicherheit könnte haptische Holographie hohes Schadpotenzial mit sich bringen: Holographisch-haptische Grenzmauern, fühlbare Personenkontrollen ohne zwischenmenschlichen Kontakt oder sexueller Missbrauch über das Internet, sind nur einige Überlegungen, die deutlich machen, dass die Antizipation von zukünftigen Risiken dieser Technologie bereits heute sinnvoll wäre.

\section{Zitierte Quellen}

Hoshi, T. et al., 2009, Touchable holography, ACM SIGGRAPH 2009 Emerging Technologies, New Orleans, Louisiana: ACM.

Ochiai, Y. et al., 2016, Fairy Lights in Femtoseconds: Aerial and Volumetric Graphics Rendered by Focused Femtosecond Laser Combined with Computational Holographic Fields, ACM Trans. Graph. 35(2), 1-14.

Page, M., 2013, Haptic Holography/Touching the Ethereal, Journal of Physics: Conference Series 415(1), 012041.

$\mathrm{Vi}, \mathrm{C}$. T. et al., 2017, Not just seeing, but also feeling art: Mid-air haptic experiences integrated in a multisensory art exhibition, International Journal of Human-Computer Studies 108, 1-14.

World Economic Forum, 2018, The Global Risks Report 2018, Geneva, Switzerland: World Economic Forum.

4 voicemag.uk/blog/296/7d-hologram-technology-could-mean-an-end-to-zoosand-captive-animals.

5 theguardian.com/world/2018/feb/15/japanese-tour-firm-offers-virtual-realityholidays-with-a-first-class-seat.

6 Die ökologische Nachhaltigkeit ist in diesem Fall an den effizienten Energieeinsatz haptisch-holographischer Technologie gekoppelt. 


\section{In die Zukunft schauen: futuristische Retina-Implantate}

Retina-Implantate sollen sehbehinderten und blinden Menschen, deren Sehnerv noch funktionstüchtig ist, das Sehen wieder ermöglichen. Neben dieser grundlegenden Aufgabe sind mit der Technologie spätestens seit den 60 er Jahre futuristische Visionen verknüpft. Ein Beispiel ist der VISOR $^{1}$, Geordi La Forges Sehhilfe aus der Serie Star Trek. Sie ermöglicht nicht nur Blinden das Sehen, sondern bietet darüber hinaus eine Reihe an Superfunktionen durch den erhöhten, wahrnehmbaren Frequenzbereich elektromagnetischer Strahlung. Anhand der zusätzlich gewonnenen visuellen Information könnten bspw. thermische Materialeigenschaften erkannt oder versteckte Hohlräume aufgefunden werden. Das mag Science Fiction sein, verweist aber auf dennoch auf neuer Möglichkeiten zur Erweiterung visueller Wahrnehmung. So könnten Retina-Implantate zukünftig das empfangene Bild über die Computer-Auge-Schnittstelle auf einen beliebigen Bildschirm übertragen oder umgekehrt Informationen aus dem Internet automatisch ins Sichtfeld einblenden. Teilweise ist diese Zukunftsvision bereits verwirklicht: erste Netzhaut-Implantate sind verfügbar, die Menschen mit geschädigter Netzhaut, aber intaktem Sehnerv helfen können (Velikay-Parel 2014). Die Implantate stimulieren elektrisch die verbliebenen Netzhautzellen und rufen eine Sehwahrnehmung hervor. Gegenwärtig können mithilfe der Technologie vollständig erblindete PatientInnen in zwei von drei Fällen Umrisse erkennen, von richtigem Sehen sind sie damit noch weit entfernt. Die Kosten der Behandlung sind enorm, die Einschränkungen der Behandlung reduzieren die behandelbaren Erkrankten auf eine kleine Zahl. ${ }^{2}$ Für die Weiterentwicklung der Technologie müssen immer wieder versuchswillige PatientInnen gefunden werden, denen in Anbetracht des gegenwärtigen Entwicklungsstandes der Medizin nicht viel Hoffnung auf Heilung gemacht werden kann. Trotzdem sind mit Retina-Implantaten hohe Zukunftserwartungen und Forschungsinvestitionen verknüpft. ${ }^{3}$ Bedenkt man die mäßigen Erfolge bei der Qualität des Implantat-gestützten Sehens, die hohen Kosten der Anschaffung, die erforderliche Geduld und Mühe bei der anfänglichen Nutzung (Lernmaßnahmen zur Interpretation visueller Information), sowie die möglichen $\mathrm{Ne}$ benwirkungen (Schmerzen, Immunreaktionen, etc.) stellt sich die Frage nach Alternativen. Ob es in Zukunft kompakte optische Geräte in Form von Augen geben wird, oder ob unsere Gesellschaft eine integrativere Lebensweise mit spezieller Aufmerksamkeit für Menschen mit Seheinschränkungen gefunden haben wird, bleibt offen. Für die Gegenwart wäre ein breiter gesellschaftlicher Diskurs zu ethischen, sozialen und ökonomischen Aspekten hoch-technologischer Gesundheitsanwendungen an der Mensch-Maschine-Schnittstelle anzuregen (das gleiche gilt bspw. für Exoskelette in der medizinischen Anwendung (siehe dazu Exoskelette).

\footnotetext{
1 memory-alpha.fandom.com/de/wiki/VISOR.

2 zeit.de/2017/51/netzhaut-chip-augenerkrankung-blinde-sehen.

3 bmwi.de/Redaktion/DE/Artikel/Hightechlight/retinaimplantat.html.
} 


\section{Zitierte Literatur}

Velikay-Parel, M., 2014, Chip im Auge. Retina Implantate - Zukunftsmusik oder bereits Realität?, sichtweisen 12/2014 - 1/2015. 


\section{High-Tech-Nahrungsmittelsysteme}

Die Weltbevölkerung wird sich nach Schätzungen der Vereinten Nationen bis 2050 auf 9,7 Milliarden Menschen vergrößern ${ }^{1}$ und zu zwei Dritteln in Städten leben. Die Produktion von Nahrungsmitteln müsste entsprechend stark gesteigert werden; ist aber im Gegenteil zunehmend bedroht durch Klimawandel, Monokulturen und eine Abhängigkeit von Importen. Eine mögliche Lösung sind geschlossene High-Tech-Nahrungsmittel-Systeme, in denen Pflanzen und Tiere unter digital kontrollierten Bedingungen ressourceneffizient gezüchtet werden und dabei weniger Platz, Wasser, Dünger und Pestizideinsatz brauchen (siehe Thema "Zellfabriken“).

Zu diesen Systemen gehört die "vertikale Landwirtschaft" (vertical farming), in der es darum geht nennenswerte Mengen an Pflanzen in mehrstöckigen Gebäuden innerhalb von Städten anzubauen (Al-Kodmany 2018). Zucht und Ernte sollen vollautomatisch erfolgen, mit Nährstoffen versorgt werden sie von einem geschlossenen Wasserkreislauf. Dabei werden unterschiedliche Systeme eingesetzt: Bei der Hydrokultur werden Pflanzen statt in Erde in anorganischen Substrat gezogen. Bei der aktuellen und effizienteren Variante, der Aeroponik, werden die freiliegenden Wurzeln der Pflanzen von Zerstäubern mit Wasser benetzt. Diese Pflanzenzucht-Systeme werden bereits mit der Zucht von Fischen kombiniert: Unter dem Begriff Aquaponik wird das Verfahren subsumiert, das die Aufzucht von Fischen in Aquakultur mit der Kultivierung von Nutzpflanzen in Hydrokultur verbindet (Love et al. 2015). Als automatisierte IndoorLandwirtschaft verbinden die Nahrungsmittelsysteme dezentrale Ansätze mit Digitalisierung und Automatisierung, wenn in Zukunft z.B. IndoorSalatfarmen komplett von Robotern und Computern betreut werden. Weitere Systeme sind intelligente, schwimmende Farmen und High-TechStadtlandwirtschaften. ${ }^{2}$ Mit den High-Tech-Nahrungsmittelsystemen kann die Ernährungssouveränität generell in Städten, in Regionen und Kontexten gestärkt werden, die aufgrund der Umweltbedingungen oder aus Platzmangel nicht ausreichend auf traditionelle Landwirtschaft zurückgreifen können, und zugleich eine Dezentralisierung der Nahrungsmittelproduktion unterstützt werden. Perspektivisch kann kleinflächiger und trotzdem effizient produziert werden; die Produktion von Nahrungsmitteln kann näher an oder sogar direkt in urbane Zentren integriert werden. Allerdings ist unklar, in welchem Ausmaß diese Systeme zur zukünftigen Welternährung beitragen können und wie die tatsächlichen Umweltwirkungen im Hinblick auf Energie-, Wasser- und Flächenverbrauch zu bewerten sind.

\footnotetext{
1 https://population.un.org/wpp/Publications/Files/WPP2019_Highlights.pdf.

2 Beispiele siehe: https://www.derstandard.at/story/2000088148977/verticalfarming-landwirtschaft-ohne-land.
} 
In Industrieländern mit ausdifferenzierter Landwirtschaft, hohem landwirtschaftlichen Knowhow und fortgeschrittener Digitalisierung und Automatisierung bietet sich die Möglichkeit, über High-Tech-Nahrungsmittelsysteme vielfältige Produkt- und Prozessinnovationen zu entwickeln.

\section{Zitierte Quellen}

Al-Kodmany, K., 2018, The Vertical Farm: A Review of Developments and Implications for the Vertical City, Buildings $8(2)<<$ Go to |S|>://WOS:000427510600012 >; auch veröffentlicht in: Buildings.

Love, D. C., Fry, J. P., Li, X. M., Hill, E. S., Genello, L., Semmens, K. und Thompson, R. E., 2015, Commercial aquaponics production and profitability: Findings from an international survey, Aquaculture 435, $67-74<<$ Go to ISI ://WOS:000345229500008 >; auch veröffentlicht in: Aquaculture. 


\section{Der gen-editierte Mensch}

\section{Zusammenfassung}

Veränderungen am menschlichen Genom sind seit der Entwicklung von CRISPR-Cas9 einfacher und präziser möglich als je zu vor. Hier müssen zwei Anwendungsfelder unterschieden werden: somatische Gentherapie, die nur das Individuum betrifft, und Keimbahn-Gentherapie, bei der Veränderungen an Nachkommen weitervererbt werden, was einen besonderen Risikofaktor darstellt. In der Keimbahntherapie führten ForscherInnen 2015 erste Versuche mit menschlichen Embryonen durch. 2018 wurden mit dem bekannten chinesischen Zwillingspaar die ersten gen-editierten Menschen geboren. Neue Therapiemethoden, beispielsweise für Erbkrankheiten oder Krebs scheinen damit in Reichweite und könnten die Medizin revolutionieren. Die internationalen Forschungsgemeinschaft ist sich aber einig: für klinische Anwendungen in der Keimbahntherapie ist es viel zu früh, das Risiko zu hoch. Die neue Technologie ist derzeit zu ungenau mit nicht abschätzbaren gesundheitlichen Nebenwirkungen für Individuen und die menschliche Art als solche, weil Genomveränderungen an Folgegenerationen vererbt werden können. Die internationale Rechtslage ist uneinheitlich, was eine überstürzte Verbreitung der Risikotechnologie befördern könnte, vor allem wenn falsche Hoffnungen verbreitet werden.

\section{Überblick zum Thema}

Mit Hilfe des gentechnischen Verfahrens CRISPR/Cas9, das auch vereinfacht als Gen-Schere, Genomchirurgie oder Gene-editing bezeichnet wird, lassen sich erstmals relativ einfach, effizient und präzise Änderungen am Genom vornehmen (siehe Thema „Künstliches Leben“). Das Verfahren revolutionierte in den letzten Jahren die Grundlagenforschung in der Biotechnologie und verspricht, dies auch in der Medizin zu tun. Das Verfahren, das auf einem bakteriellen DNA-Reparaturmechanismus nach Virusinfektionen beruht, wurde bereits 1987 beschrieben. Die genaue Funktion wurde viel später verstanden und erst um 2012 für Genomchirurgie nutzbar gemacht (Jinek et al. 2012; Doudna/Charpentier 2014). Dadurch wurden schlagartig viele gentechnische Anwendungen praktisch möglich, die vormals nur theoretisch denkbar waren. Mittlerweile gibt es mehrere Patente in zahlreichen Anwendungsfeldern wie der Therapie von Blut-, Augen-, Leber- oder Herzkrankheiten und etlicher Krebserkrankungen sowie in der Pflanzen- und Nutztierzucht (Storz 2018).

Im Gegensatz zu Pflanzen müssen in Bezug auf Menschen und Tiere zwei Anwendungsfelder unterschieden werden: die Manipulation von somatischen Zellen und die von Keimbahnzellen. Beide Formen von Eingriffen in das menschliche Erbgut können theoretisch einerseits die Behandlung von Krankheiten und andererseits die Steigerung von Fähigkeiten (Enhancement) zum Ziel haben. technische Revolution in Mikrobiologie und Medizin

zwei Anwendungsfelder: somatische Gentherapie und KeimbahnGentherapie 
somatische Gentherapie betrifft „nur" Individuen

Keimbahntherapie: Veränderungen werden vererbt

erhoffter Nutzen bei Erbkrankheiten

technisch unausgereift: sehr hohes Risiko für Nebeneffekte

Komplexität: Immunisierung gegen HIV führt zur Anfälligkeit für andere Viruserkrankungen

Auswirkungen nicht vorhersagbar
Somatische Zellen sind Körperzellen, die sich im Laufe ihres Lebens ausdifferenzieren, etwa zur Haut- oder Leberzelle und dann sterben. Gentechnische Veränderungen von Körperzellen haben damit nur Auswirkungen auf das behandelte Individuum und nicht auf die nächste Generation. Somatische Gentherapie ist relativ alt, wurde aber einige Zeit lang nicht weiterverfolgt, weil erste Versuche dramatisch gescheitert sind. Nun erhält die Form der Therapie neuen Aufwind, auch durch CRISPR/Cas9, und befindet sich bereits teilweise in der Anwendungsphase.

Keimbahntherapie verspricht die Heilung erbbedingter Krankheiten, wird aber zumindest seit den 1980er-Jahren kontrovers diskutiert. Gentechnische Eingriffe in die Keimbahn, also in das Erbgut von Geschlechtszellen (Ei und Spermium) sowie von frühen Embryos, verändern potentiell das Erbgut aller Zellen des entstehenden Organismus. Diese Änderungen werden damit auch weitervererbt, haben also Auswirkungen auf die folgenden Generationen und damit möglicherweise auf die menschliche Evolution.

Ethische Fragestellungen, die zuvor nur theoretisch verhandelt wurden, bekommen mit den seit 2015 geschaffenen Fakten neue Brisanz. Nicht zuletzt kann z.B. der therapierte Embryo dem Eingriff nicht zustimmen. Schwierig ist dabei beispielsweise, ob das Recht des gesetzlich geschützten Embryos schwerer wiegt als der Wunsch von Eltern nach einem genetisch eigenen, unbelasteten Kind (Hardt 2019).

Genomchirurgie mit CRISPR/Cas9 ist zwar viel effektiver und genauer als andere bis dato bekannte Verfahren, birgt aber trotzdem Unsicherheiten mit potentiell weitreichenden Folgen (Hardt 2019). Oft wird das Verfahren so dargestellt, als sei es höchst präzise und auch der Beiname „Editierung“ weckt die Assoziation eines sauberen „Löschens“ und „Einfügens“. Wie bei allen biologischen Prozessen gibt es aber auch bei diesem Verfahren Abweichungen bezüglich des erwarteten Ergebnisses: es kann also vorkommen das die gewünschte genetische Sequenz gar nicht, an falscher Stelle oder nicht in allen Zellen eingebaut oder herausgeschnitten wird (sogenannte Off-target-Effekte).

Im Genom ist beispielsweise ein Gen oft an der Ausprägung mehrerer Eigenschaften beteilig und gleichzeitig mehrere Gene an einer Eigenschaft. Bestimmten Genveränderungen haben deshalb nicht immer nur die gewünschten Effekte, sondern können fatale Nebenwirkungen haben: Im Fall der chinesischen Zwillinge sollte die Ausprägung eines Eiweißes an das der HI-Virus andockt verhindert werden, um so HIV Immunität zu erreichen. Erstens kam es bei der Behandlung zu Mosaikeffekten, was bedeutet, dass zumindest bei einem der Zwillinge das entsprechende Gen nicht gänzlich deaktiviert wurde. Zweitens spielt das Eiweiß bei Schutz von Lunge, Leber und Gehirn bei anderen schweren Infektionen eine Rolle (Cyranoski 2018).

Solche unerwünschten Nebeneffekte sind außerdem schwer vorherzusagen, da auch die Techniken zur Erkennung der Off-target-Effekte noch unausgereift sind. Welche Auswirkungen solche Mutationen hervorrufen können, ist ebenfalls nicht immer abschätzbar. Manchmal zeigen die feh- 
lerhaft verbauten oder geschnittenen Sequenzen keine direkten Auswirkungen auf den Phänotyp, also das äußere Erscheinungsbild, des behandelten Organismus, allerdings gibt es auch keine Langzeitstudien, da derzeit die zu Forschungszwecken behandelten menschlichen Embryonen meist nach spätestens sieben Tagen zerstört werden (Hardt 2019).

In Anlehnung an den Missbrauch herkömmlicher pharmazeutischer Produkte kann auch Missbrauch von gentherapeutischen Verfahren oder Produkten nicht ausgeschlossen werden. Sollten etwa Produkte zur Behandlung von Muskel- oder kognitiven Störungen zugelassen werden, könnten sie zur Verbesserung der Leistungsfähigkeit von Einzelpersonen eingesetzt werden. Generell könnte durch die technische Machbarkeit des Enhancements ein gesellschaftlicher Optimierungs- und Leistungsdruck entstehen, soziale Ungerechtigkeiten könnten verschärft oder Veränderungen könnten zwangsweise verordnet werden (EASAC 2017; Hardt 2019). Aus den genannten Gründen ist aber derzeit eine Beeinflussung von hochkomplexen Eigenschaften wie Intelligenz nicht absehbar.

Auch wären gentechnische Anpassungen etwa von Soldatlnnen als Gegenmaßnahme zu biologischen Waffen denkbar. Die weltweite Regulierung militärischer Forschung und Entwicklung in diesem Bereich ist herausfordernd bis unmöglich. Auch ein Missbrauch der Technologie zur Bearbeitung des menschlichen Genoms in der Do-it-yourself (DIY) Biologie ist denkbar, momentan gibt es hierfür aber keine Evidenz und das Risiko wird zumindest für die nahe Zukunft als niedrig eingeschätzt (Fears/IAP 2017).

Absehbarer ist aber vermehrter Medizintourismus in Bezug auf Therapien, sollte der Zugang zur Technologie in verschiedenen Ländern sehr unterschiedlich reguliert sein. Auch wenn die technischen Voraussetzungen noch gar nicht gegeben sind, könnten aus Geschäftsinteresse unbegründete Hoffnungen geweckt werden, gerade in Ländern mit rechtlichen Graubereichen.

Führende WissenschafterInnen, unter innen Emmanuelle Charpentier, die die Anwendung von CRISPR/Cas9 als Genschere als erste erfolgreich demonstrierte, fordern ein weltweites Moratorium für die klinische Anwendung des Verfahrens in der menschlichen Keimbahn. Sie fordern einen internationalen Rahmen, in dem sich alle Länder unter Wahrung ihrer nationalen Souveränität freiwillig verpflichten, die klinische Verwendung nicht zu genehmigen. So solle Zeit gewonnen werden, um wichtige technische, medizinische, soziale, ethische und moralische Fragestellungen ausreichend zu erörtern (Lander et al. 2019).

Zurzeit fehlt noch das nötige Wissen, um Risiken und Nutzen ausreichend abzuwägen, und damit kann nicht entschieden werden, ob und in welcher Form Keimbahntherapie überhaupt zulässig sein kann. Dieses Moratorium soll jedoch nicht Erbgutveränderungen in der Keimbahn zu Forschungszwecken betreffen, solange solche Embryonen nicht ausgetragen werden. Genauso ausgenommen soll Gentherapie an somatischen Zellen sein, also Erbgutveränderungen, die nicht weitervererbt werden können (Lander et al. 2019).
Sicherheitsrisiken: potentieller Missbrauch, Optimierungsdruck, Zwangsverordnungen

Sicherheitsrisiken: Dual Use und DIY-Biologie

vermehrter

Medizintourismus und Kriminalität

weltweites Moratorium für Keimbahneingriffe gefordert...

...wichtige technische, medizinische, soziale, ethische und moralische Fragen ungeklärt 
rigorose Evaluierung notwendig

internationale

Rechtslage nicht einheitlich

USA, China und Großbritannien erlauben Keimbahnveränderung zur Forschung

Reformbedarf für kohärente regulatorischen Rahmenbedingungen
In Bezug auf die somatische Gentherapie besteht hoher Bedarf, die Risiken, z.B. durch ungenaue Editierung, besser zu verstehen, auch um den potentiellen Nutzen jeder Anwendung abzuschätzen. Hierfür ist eine rigorose Evaluierung innerhalb des bestehenden und sich weiterentwickelnden Rechtsrahmens für die Gen- und Zelltherapie durch die europäischen und nationalen Arzneimittel-Agenturen notwendig. Bei Keimbahneingriffen ist das Risiko ungleich höher, da schädliche Auswirkungen nicht nur für die behandelten Individuen, sondern auch für zukünftige Generationen abzuschätzen sind. Hierzu wird auch ein breiter gesellschaftlicher Diskurs und Konsens benötigt (EASAC 2017).

\section{Relevanz des Themas für das Parlament und für Österreich}

Die Rechtslage ist derzeit sehr unterschiedlich. Während viele Länder Keimbahntherapie oder Versuche mit Embryonen explizit verbieten, erlauben dies andere; in dritten fehlt eine gesetzliche Regelung (ITA 2016). Während ForscherInnen 2015 die ersten Versuche mit CRISPR/Cas9 an menschlichen Embryonen veröffentlichten, wurden bereits 2018 - wenn auch unter Umgehung aller Gesetzte und Ethikrichtlinien - mit dem bekannten chinesischen Zwillingspaar die ersten gen-editierten Menschen geboren.

Derzeit erlauben die USA, China und Großbritannien Keimbahnveränderungen zu Forschungszwecken, alle anderen europäischen Länder verbieten diese. Diese Uneinheitlichkeit befördert Konkurrenzängste, die Liberalisierung nationaler Embryonenschutzgesetze, etwa in Deutschland, steht zur Debatte. Oft sind diese Gesetze auch nicht eindeutig bzw. decken neue technologische Möglichkeiten nicht ab. So werden beispielsweise der Zellkerntransfer, also der Ersatz des ganzen Kerns (und damit des gesamten enthaltenen Genoms), nicht erfasst, da keine Erbgutveränderung stattfindet (Hardt 2019).

So können Graubereiche entstehen, die zu einer überstürzten Verbreitung und klinischen Anwendung der Technologie führen können. Damit wird eine internationale Kooperation zur Reform der regulatorischen Rahmenbedingungen zumindest in der EU, besser weltweit, notwendig.

\section{Vorschlag weiteres Vorgehen}

Es bietet sich an, die umfassende Debatte, die lange vor der Erfindung der modernen CRISPR/Cas9-Genschere sozusagen theoretisch geführt wurde, für die aktuelle Diskussion angesichts der nun vorhandenen tatsächlichen technischen Möglichkeiten fruchtbar zu machen. Dabei würde auch aufgezeigt werden, welche Fragestellungen in der früheren Debatte noch gar nicht gestellt werden konnten (etwa im Zusammenhang mit der DIY-Bewegung und den damit grundsätzlich neuen Verbreitungs- und Kontrollperspektiven). Das könnte auch die Grundlage für einen partizipativen Prozess sein und eine längere parlamentarische Diskussion im Rahmen einer Enquete-Kommission bilden. 


\section{Zitierte Literatur}

Cyranoski, D., 2018, Baby gene edits could affect a range of traits, Nature, nature.com/articles/d41586-018-07713-2.

Doudna, J. A. und Charpentier, E., 2014, The new frontier of genome engineering with CRISPR-Cas9, Science 346(6213), 1077.

EASAC, 2017, Genome editing: scientific opportunities, public interests and policy options in the European Union, European Academies Advisory Council: EASAC.

Fears, R. und IAP (PArtnership, I.), 2017, Assessing the Security Implications of Genome Editing Technology. Report of an international workshop, im Auftrag von: Partnership, I., Interacademy Partnership, Herrenhausen, Germany.

Hardt, A., 2019, Technikfolgenabschätzung des CRISPR/Cas-Systems, Über die Anwendung in der menschlichen Keimbahn, degruyter.com/view/product/510634.

ITA, 2016, Genkorrekturen - neue Technik, altes Risiko? ITA-Dossier Nr. 21 (Mai 2016; Autorlnnen: Helge Torgersen, Karen Kastenhofer), Wien, epub.oeaw.ac.at/ita/ita-dossiers/ita-dossier021.pdf.

Jinek, M., Chylinski, K., Fonfara, I., Hauer, M., Doudna, J. A. und Charpentier, E., 2012, A Programmable Dual-RNA-Guided DNA Endonuclease in Adaptive Bacterial Immunity, Science 337(6096), 816-821, science.sciencemag.org/content/sci/337/6096/816.full.pdf.

Lander, E., Baylis, F., Zhang, F., Charpentier, E. und Berg, P., 2019, Adopt a moratorium on heritable genome editing, Nature 567, 165-168.

Storz, U., 2018, CRISPR Cas9 - Licensing the unlicensable, J Biotechnol 265, 86-92, ncbi.nlm.nih.gov/pubmed/29154806. 


\section{„Right to Challenge" als alternatives Bewertungsverfahren für die Sharing Economy}

\section{Zusammenfassung}

Die Sharing Economy baut auf das Prinzip „Teilen statt Besitzen“ und ermöglicht neue Geschäftsmodelle, die sich mit Hilfe von digitalen Plattformen schnell durchgesetzt haben. Neben den erwarteten Vorteilen, wie $\mathrm{CO}_{2}$-Einsparungen und höhere Ressourceneffizienz, kann die SharingEconomy aber auch negative Wirkungen haben: Sekundäreffekte überkompensieren die erhofften Vorteile, die soziale Absicherung der Anbieter ist $u$. U. gering, und die Plattformanbieter können sich regulativen Anforderungen entziehen. Zudem entwickeln sich Sharing Plattformen sehr rasant und ziehen dabei unerwartete Folgen nach sich. Welche Strategien entwirft die Politik für den Umgang mit den Effekten der Sharing Economy? Und wie können solche Effekte in der Behandlung von möglichen Technikfolgen berücksichtigt werden?

\section{Überblick zum Thema}

Der Aufstieg der Sharing Economy als einem speziellen Teilbereich der Plattformökonomie ging einher mit optimistischen Erwartungen hinsichtlich möglicher Umwelt- und sozialer Effekte dieses Konzepts. Der Sharing Economy liegt das Prinzip zugrunde, dass KonsumentInnen sich gegenseitig vorübergehenden Zugang zu nicht ausgelasteten physischen Vermögenswerten, sogenannten „physical assets“ gewähren, u. U. für Geld. ${ }^{1}$ Diese Möglichkeiten des Teilens von "physical assets“ im Austausch für Geld wurde durch die Einführung digitaler Plattformen deutlich vereinfacht und hat dadurch erst die allseits bekannten Beispiele der Sharing Economy wie Uber oder Airbnb ermöglicht. Allerdings mag auch der Reiz des Neuen zum steilen Aufstieg der Sharing Economy beigetragen haben, obwohl es nicht-digitale Vorläufer bereits seit vielen Jahren gegeben hat. Dabei kann zwischen drei Grundtypen unterschieden werden:

- "Second-hand economy", d. h. der An- und Verkauf gebrauchter Produkte durch private und professionelle Beteiligte, der durch Plattformen wie ebay oder willhaben deutlich erleichtert wurde.

- „Product-service economy“, bei der die temporäre Nutzung von Produkten, die im Besitz professioneller Anbieter verbleiben im Vordergrund steht; ein Modell, dass wir alle von der Autovermietung kennen.

- "On-demand economy", bei der es um das Anbieten und die Nutzung von Dienstleistungen und nicht von Produkten geht; häufig wird hierfür auch der Begriff der "gig-economy“ verwendet (siehe Dienstleistung 4.0).
Bessere Nutzung von nicht ausgelasteten "physical assets" ermöglichen

Drei Grundtypen der digitalen Sharing Economy

\footnotetext{
1 „consumers granting each other temporary access to under-utilized physical
} assets (,idle capacity“), possibly for money“ Frenken/Schor (2017). 
Sekundär-Effekte der Sharing-Praxis

Monopolbildung und Netzwerkexternalitäten

Datenzugang und Datenschutz
Sharing gibt es also schon sehr lange, aber erst die digitalen Plattformen haben Sharing-Dienste für ein breites Spektrum neuer und häufig privater Akteure erschlossen.

\section{Unerwartete Effekte der digitalen Plattformen}

Die Sharing Economy hat in den letzten Jahren eine rasante Entwicklung genommen und neben der geteilten Nutzung von Produkten insbesondere Dienstleistungsangebote in den Bereichen Mobilität, Tourismus und Arbeitsmärkte verändert. Im Zuge dieser Entwicklung sind eine Reihe von Effekten deutlich geworden, die zumindest als ambivalent anzusehen sind. So führen Praktiken des Sharing häufig zu Sekundär-Effekten in benachbarten Bereichen, die zu interessanten gesamt- und einzelwirtschaftlichen Phänomenen führen. Hotels leiden unter dem Anwachsen des Marktes für Airbnb-Wohnungen, insbesondere in einigen wichtigen touristischen Destinationen. Car-Sharing-Modelle führen nicht notwendigerweise zu weniger Verkehr, weil sie häufig Wege ersetzen, die ansonsten zu Fuß oder öffentlich gemacht worden wären. Die zunächst plausibel erscheinenden positiven Umwelteffekte von Car-Sharing werden dadurch zumindest teilweise kompensiert (siehe Offene Mobilitätsplattformen). Auch die Erwartungen hinsichtlich der positiven ökonomischen Effekte für die Anbieter von Sharing-Leistungen haben sich nur zum Teil erfült. Dies ist insbesondere darauf zurückzuführen, dass die Anbieter von Sharing-Plattformen eine inhärente Tendenz zur Monopolbildung besitzen und aufgrund ihrer Marktmacht einen erheblichen Teil der Gewinne aus Sharing-Geschäften abschöpfen können. Diese Monopolbildung hat ihre Ursache in sogenannten Netzwerkexternalitäten, aufgrund derer - ähnlich wie beim Telefon der Nutzen einer Plattform für die Leistungsanbieter ebenso wie für die Nutzer mit der Reichweite der Plattform steigt. ${ }^{2}$ Rasch wachsende Plattformen werden daher immer attraktiver, wodurch die Macht der Plattformbetreiber gegenüber den Dienstleistungsanbietern (und damit die Möglichkeiten höhere Margen einzubehalten) steigt. ${ }^{3}$ Hinzu kommt, dass die durch die Sharing-Aktivitäten der Dienstleistungsanbieter gewonnenen Daten den Plattformanbietern gehören und von diesen für die Entwicklung neuer und die Verbesserung bestehender Geschäftsmodelle genutzt werden können. Dies wirft außerdem Fragen nach dem Datenschutz auf.

Insgesamt bieten Sharing-Modelle den Anbietern von Leistungen wichtige wirtschaftliche Chancen, um ihre „physical assets“ besser nutzen und verwerten zu können. Dies setzt allerdings voraus, dass man überhaupt derartige Assets besitzt und über die erforderlichen Fähigkeiten verfügt um Sharing-Leistungen anbieten zu können. In der Praxis hat sich gezeigt,

2 Plattformen können als Netzwerke verstanden werden, in denen die Anbieter und Nutzer von Leistungen über den Plattformbetreiber verknüpft sind. Das Netzwerk als Ganzes bietet Vorteile für alle: Je größer die Anzahl der Anbieter, desto attraktiver ist das Angebot für die Nutzer und desto größer sind auch die Anreize für neue Anbieter sich der Plattform anzuschließen.

3 Das untermauert ein bekannter, kartellrechtlicher Disput zwischen einem Dienstleistungsanbieter und dem Plattformbetreiber: theverge.com/2020/8/13/ 21366438/apple-fortnite-ios-app-store-violations-epic-payments. 
dass Sharing-Modelle die Ungleichheit in einer Gesellschaft tendenziell verstärken (Schor 2017). Zugleich haben Sharing-Konzepte für die Anbieter von Leistungen neben den wirtschaftlichen Effekten häufig auch einen sozialen Nutzen. Das hat sich insbesondere bei Airbnb gezeigt, wo häufig das Interesse an neuen sozialen Kontakten die Anbieter motiviert, ihr Haus oder ihre Wohnung temporär zu teilen. Allerdings nimmt aufgrund der zunehmenden Professionalisierung von Airbnb Angeboten die Bedeutung dieser sozialen Kontakte als Motivation ab. Insbesondere in den USA hat sich zudem zeigen lassen, dass Airbnb nicht frei von Diskriminierungen bestimmter sozialer Gruppen ist, z. B. im Hinblick auf Bewertungen und Preise (Cansoy/Schor 2017).

\section{Ansätze für die bewusste Gestaltung der Sharing Economy}

Die digitale Sharing Economy entwickelt sich rasant. Wenige Jahre haben gereicht, um sie zu einem relevanten und zum Teil auch umstrittenen Phänomen zu machen. Zu Beginn dieser Entwicklung waren viele der Effekte unklar und schlichtweg unbekannt. Die Einführung von Sharing Modellen erfolgte in der Regel frei von spezifischen Regulierungen, bzw. in einer regulativen Grauzone, weil sie sich nicht ohne Weiteres in existierenden Leistungskategorien fassen ließen. Man kann hier von Großexperimenten mit offenem Ausgang sprechen, deren Wirkungen sich erst in der Umsetzung gezeigt haben und die man in Echtzeit beobachten konnte. Die Regulierung derartiger Sharing Modelle erfolgte daher meist reaktiv. Heftige Auseinandersetzungen über Uber sind aus vielen Ländern bekannt, in denen sich das hochregulierte Taxigewerbe bedroht sah. Städte beklagen sich über entgangene Steuern und Abgaben von AirB'nB-Anbietern, die Hotellerie über eine wenig regulierte Konkurrenz, Anwohner über Lärmbelästigung. Allerdings fällt bislang der Nachweis positiver wie negativer Sekundäreffekte schwer, weil die privaten Unternehmen, die die Plattformen betreiben, nur schwer zur Preisgabe ihrer Daten verpflichtet werden können. Im Falle von AirBn'B sind einige Städte dazu übergegangen, genauer auf die Unterscheidungen von professionellen und nicht-professionellen Angeboten zu achten, und haben Begrenzungen bei der Anzahl von Nächtigungen im Sharing-Modus eingeführt. Damit soll gewährleistet werden, dass - der Definition von Sharing Economy folgend - lediglich eine temporäre Nutzung möglich ist. Die Idee dahinter ist, dass die Chancen der Sharing Economy für Private ohne weitreichende regulative Restriktionen erhalten bleiben sollen, während der professionelle Vertrieb von Leistungen den üblichen Standards vergleichbarer Berufsgruppen folgen soll. Diese verschiedenen Beispiele zeigen, dass die Effekte der Sharing Economy nach wie vor vielen Unsicherheiten unterliegen. Aufgrund der dynamischen Entwicklung der Plattformen und des Nutzer- und Anbieterverhaltens besteht auch ein Bedarf an einer kontinuierlichen Neubewertung und ggf. dem Nachjustieren von rechtlichen und anderen Regelungen.
Tendenz zur

Verstärkung gesellschaftlicher Ungleichheit ...

... trotz sozialen

Nutzens für die Leistungsanbieter

Rasante Entwicklung der Sharing Economy

Regulative Grauzonen und EchtzeitExperimente mit offenem Ausgang

Professionelle vs. nicht-professionelle Angebote

Unsichere Effekte und dynamische Entwicklung

Bedarf an

kontinuierlicher

Neubewertung 


\author{
Alternative \\ Plattform-Modelle: \\ Kooperativen und \\ öffentliche \\ Sharing-Dienste
}

Mangelnder Zugang zu Nutzungsdaten für Forschungszwecke

Nachgelagerte Technikfolgenabschätzung ...

... in Kombination mit dem, Right to Challenge'

\section{Welche grundsätzlichen Alternativen gibt es?}

Über die verschiedenen Wirkungsdimensionen hinaus gibt es auch grundsätzliche Fragen nach der Architektur von Sharing Plattformen. Ist das Modell der zur Monopolisierung und Machtkonzentration neigenden Plattformbetreiber unvermeidbar oder gibt es Alternativen dazu? Ein wichtiges Leitprinzip für alternative Modelle wäre zunächst eine höhere Transparenz. Erst der Zugang zu Nutzerdaten (der derzeit unter Verweis auf Datenschutz von den Betreibern sehr restriktiv gehandhabt wird) kann die empirische Basis für eine fundierte Bewertung der positiven und negativen Effekte der Sharing Economy liefern; und damit auch für evidenzbasiertes korrektives Eingreifen der Politik, um Fehlentwicklungen zu korrigieren und Missbrauch zu vermeiden. Tatsächlich gibt es Experimente mit alternativen offenen Plattform-Modellen, die auf dem Modell von Kooperativen basieren und die Gewinne unter den Beteiligten aufteilen, bzw. in die Weiterentwicklung der Plattform investieren (Scholz 2016). Auch gibt es Überlegungen öffentlich betriebene Plattformen zu etablieren, die nichtkommerzielle Sharing-Dienste anbieten Allerdings kämpfen diese neuen Plattformen mit großen Hürden, um sich gegen die etablierten Großen in ihren angestammten Feldern profilieren zu können. Auch geben sich einige der kommerziellen Plattformen inzwischen offener für ein höheres Maß an Transparenz; dies gepaart mit Eigeninteresse, um einschneidende Regulierungen zu vermeiden.

\section{Weiteres Vorgehen}

Um zu gewährleisten, dass digitale Sharing Plattformen eine gute Entwicklung nehmen und negative Sekundäreffekte vermieden werden, wäre ein transparenter Zugang zu Nutzungsdaten wichtig, der aber seitens der privaten Betreiber häufig auch für Forschungszwecke verwehrt wird. Dies führt insofern zu einem Problem als TA-Analysen erschwert werden, weil die empirische Basis für eine Bewertung fehlt. Weder ist eine detaillierte wissenschaftliche Analyse der Effekte der Sharing Plattformen möglich noch eine fundierte partizipative Auseinandersetzung mit ihren normativen Implikationen. Hinzu kommt die Geschwindigkeit, mit der Sharing Plattformen an Bedeutung gewinnen. Dies führt dazu, dass eine antizipierende Bewertung von Technikfolgen kaum möglich ist, sondern lediglich eine nachgelagerte, d. h. nachdem die Plattformen bereits eingeführt wurden (Frenken/Schor 2017). Angesichts dieser Herausforderungen wird in den Niederlanden das Prinzip des 'Right to Challenge' von Regulierungen als alternativer Politikansatz und als Ergänzung zur nachgelagerten Technikfolgenbewertung erwogen (Frenken/Pelzer 2020). Dabei werden zunächst anspruchsvolle regulative Ziele (z. B. soziale Standards von Leistungsanbietern) und konkrete Anforderungen (z. B. Arbeitszeitregelungen) für neue Plattformen definiert. Die Plattformbetreiber können jedoch eine befristete Ausnahme von den spezifischen Anforderungen erlangen, wenn sie glaubhaft machen, dass sie die Ziele der Regulierung auch auf anderem Weg erfüllen können. Nach Ablauf der Befristung wird eine ex-post Überprüfung in der Form einer nachgelagerten Technikfolgenbewertung vorgenom- 
men, ob die Ziele tatsächlich eingehalten wurden und ob für die weitere Zukunft Auflagen gemacht werden müssen. Diese Erfahrungen legen nahe, dass neben neuen Verfahren des Foresight und der TA für sich rasch entwickelnde Technologien (z. B. begleitende TA oder transformativer Foresight) auch komplementäre Ansätze wie das „Right to Challenge“ in Österreich erprobt werden könnten, um negative Effekte digitaler Plattformen zu mildern und zugleich deren Potenziale zu nutzen. Zuvor müssen allerdings die rechtlichen Voraussetzungen geschaffen werden, um derartige experimentelle Verfahren zu ermöglichen (siehe Regulatorische Experimentierräume).

\section{Zitierte Literatur}

Cansoy, M. und Schor, J. B., 2017, Who Gets to Share in the Sharing Economy: Racial Discrimination on Airbnb, Boston College, Working Paper.

Frenken, K. und Pelzer, P., 2020, Reverse Technology Assessment in the Age of the Platform Economy, Built Environment 46(1), 22-27, doi.org/10.2148/benv.46.1.22.

Frenken, K. und Schor, J., 2017, Putting the sharing economy into perspective, Environmental Innovation and Societal Transitions 23, 3-10, sciencedirect.com/science/article/pii/S2210422417300114.

Scholz, T., 2016, Platform Cooperativism. Challenging the Corporate Sharing Economy, New York: Rosa Luxemburg Stiftung, New York Office rosalux-nyc.org/wpcontent/files_mf/scholz_platformcoop_5.9.2016.pdf.

Schor, J. B., 2017, Does the sharing economy increase inequality within the eighty percent?: findings from a qualitative study of platform providers, Cambridge Journal of Regions, Economy and Society 10(2), 263-279, doi.org/10.1093/cjres/rsw047. 


\section{Künstliches Leben}

\section{Zusammenfassung}

Unter dem Begriff „Künstliches Leben“ werden Ansätze in den Biowissenschaften und IKT zusammengefasst, die durch Simulation natürlicher Lebensprozesse zu deren Verständnis beitragen und durch Anwendungen künstliche Formen von Leben zu erschaffen suchen. Während früher mechanische Modelle von Robotern im Mittelpunkt standen, sind es heute auch biologische Prozesse und Computermodelle. Die biologischen Ansätze zur Erschaffung von künstlichem Leben werden von zwei verschiedenen Richtungen vorangetrieben: Während mit der Synthetischen Biologie (Synbio) Leben quasi ,am Reißbrett' entworfen wird, um Zellen oder Organismen neu zu konstruieren, gehen Ansätze wie das Gene Editing (Gen-Schere; CRISPR/Cas9) von existierenden Organismen aus, an denen neue Eigenschaften implementiert werden sollen. Ziel ist in beiden Ansätzen, Lebensformen zielgerichtet zu entwerfen oder zu transformieren. Nachdem Ende 2018 publik wurde, dass in China Zwillinge geboren wurden, die angeblich gegen HIV resistent sind, weil ein Forscher ihr Erbgut mit der Gen-Schere CRISPR/Cas9 verändert hat, ist eine breitere Debatte um künstliches Leben entfacht worden (siehe Thema "CRISPR/Cas9Mensch"). ${ }^{1}$

\section{Überblick zum Thema}

Mit Synbio werden biologische Systeme geschaffen, die in der Natur nicht Synthetische Biologie vorkommen, so dass die interdisziplinären Biowissenschaften Moleküle, Zellen und Organismen entwerfen, die vollständig neue Eigenschaften haben können. Bei Synbio sind komplette synthetische Genome das Ziel, wobei es primär um die Konstruktion von Minimalzellen aus biochemischen Grundkomponenten geht. Zu den möglichen Anwendungen gehören neue Chemikalien und Treibstoffe aus nachwachsenden Rohstoffen, wie auch Mikroorganismen zum Detektieren und Sanieren von Schadstoffkontaminationen in der Umwelt (Purnick/Weiss 2009). Während vollständig neue Organismen in ihrer mittelfristigen Machbarkeit unklar sind, zeichnet sich die Tendenz ab, künstliches Leben durch Veränderung bestehender Organismen zu erzielen, wie z. B. abgeschwächte Viren für sichere und effektive Impfstoffe.

1 Siehe z. B.: derstandard.at/2000093129040/Der-Tabubruch-der-erstenmanipulierten-Kinder\#, vgl. (Regalado, 2019). 
Gene Editing: CRISPR/Cas9

hohes Potenzial
Mit dem CRISPR/Cas9-System², das seit einigen Jahren die Gentechnik von Grund auf verändert, wird es möglich, das Erbgut gezielt zu verändern (Doudna/Charpentier 2014). CRISPR wird auch als "Gen-Schere“ bezeichnet, da dieses Verfahren des Gene Editing verspricht, beliebige, hochpräzise Änderungen am Genom vornehmen zu können.

Insgesamt ist die Abgrenzung der neuen Methoden und Ansätze zu Konzepten und Methoden der etablierten Gentechnik, Systembiologie, Molekularbiologie und Biotechnologie schwierig und umstritten. Damit ist auch die Einschätzung zukünftiger spezifischer Anwendungspotenziale der Synbio schwierig, denn in den etablierten Zweigen der Biowissenschaften sind ebenfalls eine Vielzahl von Ansätzen bekannt, neue biologische Funktionen in Organismen zu integrieren (z. B. Metabolic-Engineering zur gezielten Optimierung vorhandener Stoffwechselwege).

Den Ansätzen in Hinblick auf künstliches Leben wird ein hohes Potenzial zugeschrieben, neue Verfahren in der weißen, roten und grünen Biotechnologie zu ermöglichen, die Systemtransformation hin zu erneuerbaren Rohstoffen zu unterstützen, industrielle Prozesse in Richtung Bioökonomie zu treiben und neue Wege in der Biomedizintechnik anzugehen. Die über die bisherige Systembiologie, Gen- und Biotechnologie hinausgehenden Potenziale des biologischen künstlichen Lebens liegen mittelfristig weniger in fundamental neuen Konzepten, sondern vielmehr in der Kombination technowissenschaftlicher Entwicklungen und in den daraus resultierenden Synergien (vgl. Mackenzie 2010). Technologien zur Synthese und dem Zusammenfügen von Genomteilen, das computergestützte Modellieren von komplexen Funktionen (siehe Thema „Xenobots“), automatisierte genetische Manipulationsmöglichkeiten und die molekularen Werkzeuge zum einfachen und schnellen Einbringen gezielter Veränderung in Genome (Genome Editing, CRISPR/Cas9) bringen in ihrer Kombination ein hohes Potential an rasanten Veränderungen.

Die Tendenz, synthetische Mikroorganismen über den Begriff des künstlichen Lebens zu einem medialen Thema zu machen, verweist einerseits auf die umfassenden Versprechen, die mit der Technologie verbunden sind, trägt aber auch dazu bei, Synbio mit einem aufgeladenen Deutungsrahmen zu verknüpfen. In Bezug auf CRISPR/Cas9 hat die Nachricht von genveränderten Zwillingen in China als Schritt hin zu Designer-Babys diese Debatte ausgelöst und zur Forderung nach einem Moratorium geführt.

2 Die englische Abkürzung CRISPR bezeichnet Clustered Regularly Interspaced Short Palindromic Repeats, sich wiederholende DNA-Sequenzen, die im Erbgut vieler Bakterien auftreten und in ihrem Abwehrsystem eine wichtige Rolle spielen. Wenn ein Virus in ein Bakterium eindringt, baut die Zelle Teile der Virus-DNA in ihre CRISPR-Struktur ein und gelangt erneut ein Virus mit dieser DNA in das Bakterium, wird es mit Hilfe der CRISPR-Abschnitte erkannt. Cas9 ist die Abkürzung von CRISPR-associated protein 9. Das Cas9-Enzym dockt an einen erkannten DNA-Abschnitt an und kann so virale DNA zerschneiden. 


\section{Relevanz des Themas für das Parlament und für Österreich}

Fragen der biologischen Sicherheit haben die Entwicklung von Synbio von Beginn an begleitet (ETC Group 2010; Bennett et al. 2009, GómezTatay \& Hernández-Andreu, 2019). Toxizität, Ausbreitungsverhalten und Überlebensfähigkeit sind weitgehend unbekannt, sodass die Fragen der Biosafety darauf ausgerichtet sind, festzustellen, ob aktuelle und mittelfristige Entwicklungen von den geltenden Regulierungen (für Arzneimittel, Medizinprodukte, Chemikalien, gentechnisch veränderte Organismen) angemessen erfasst sind, oder etablierte Verfahren der Risikoabschätzung und des Risikomanagements einer Anpassung bedürfen. Die Frage der Biosecurity bezieht sich auf mögliche illegale Nutzung (Biocrime) oder die Nutzung zu Zwecken des Terrors (Bioterror). Ein aktueller Bedarf zur Überarbeitung der Risikoregulierung lässt sich auf europäischer Ebene zurzeit nicht feststellen.

Die mediale Berichterstattung fokussiert auf spektakuläre Forschungsberichte wie beispielsweise die Erfindung der ,künstlichen Bakterienzelle Synthia' durch das Team von Craig Venter, deren Konsequenzen noch unklar sind, oder auch die Designerbabys durch Genome Editing.

Insbesondere Synbio ist stark mit früher Begleitforschung verbunden (z. B. Calvert/Martin 2009). Im Zuge einer erhöhten Aufmerksamkeit für Ansätze einer verantwortungsvollen Forschung und Innovation (Responsible Research and Innovation - RRI) ist Synbio ein Paradebeispiel für umfassende Reflexions- und Beteiligungsprozesse in einer frühen Entwicklungsphase. Inwieweit Konzepte wie RRI dafür genutzt werden können, aktuell neue Biotechnologien frühzeitig auf breit gesellschaftliche akzeptierte und gewünschte Innovationspfade zu führen, ist derzeit noch nicht erprobt.

Die aktuellen Ansätze zur Schaffung „künstlichen Lebens“ sind politikfeldübergreifend relevant, da einerseits Innovationspotentiale für die österreichische Industrie vorhanden sind (weiße Biotech), aber zugleich in der Forschungspolitik und in Bezug auf ethische Fragen neue Aspekte relevant werden (z. B. „Designerbabies“, genetisch „neu“ modifizierte Organismen).

Selbst bei den Ansätzen zur Schaffung künstlichen Lebens einfachster Organismen handelt es sich um gesellschaftlich potenziell umstrittene Technologien, die gerade nicht isoliert auf ihre Technologiepotenziale hin untersucht, sondern politikfeldübergreifend daraufhin überprüft werden sollten, welche Problemlösungspotentiale sie im Vergleich zu anderen Ansätzen haben. Eine Beteiligung gesellschaftlicher Akteure außerhalb von Wissenschaft und über die traditionellen Akteure des Innovationssystems hinaus wäre in diesem Fall von hoher Bedeutung: Akteure z. B. aus der KI-Forschung, dem Gesundheitssystem, der Landwirtschaft aber auch der Do-it-yourself-Bewegung wären wichtig, um sowohl deren Anforderungen an die Technologie als auch den Erfahrungshorizont zur Einordnung der Technologien einzubinden.
Bio-Sicherheit

öffentlicher Diskurs

Governance

politikfeldübergreifende

Relevanz

Förderpolitik 
Regulierung Der Europäische Gerichtshof hat im Sommer 2018 ein überraschend begrenzendes Urteil zu CRISPR/Cas9 in Bezug auf die Landwirtschaft gefällt, dass die strengen Gentechnik-Richtlinien auch für die neuen Methoden gelten. ${ }^{3}$ Für Experimente in Richtung menschlichen künstlichen Lebens durch CRISPR/Cas9 werden ein Moratorium und globale Regelungen gefordert (Lander et al. 2019).

\section{Vorschlag weiteres Vorgehen}

Die dargestellten Themenkomplexe könnten im Überblick in einer Kurzstudie bearbeitet werden, die den Stand der internationalen Diskussion darstellt. Für eine umfassende Analyse, die die unterschiedlichen wissenschaftlich-technischen Zukunftsoptionen mit einer Analyse der Situation in Österreich verbindet, wäre eine Langstudie sinnvoll. In diesem Rahmen wäre neben der Aufarbeitung der wissenschaftlichen Literatur eine Identifikation der relevanten Stakeholder-Gruppen angemessen, die an der Entwicklung von Zukunftsoptionen beteiligt werden können (z. B. SzenarioProzess). Das breite Thema des künstlichen Lebens wäre im engen Zusammenhang mit dem Moratorium und der globalen Einigung hinsichtlich zukünftiger Erbguteingriffe am Menschen zu untersuchen.

\section{Zitierte Literatur}

Bennett, G., Gilman, N., Stavrianakis, A. und Rabinow, P., 2009, From synthetic biology to biohacking: are we prepared?, Nat Biotechnol 27(12), 1109-1111.

Calvert, J. und Martin, P., 2009, The role of social scientists in synthetic biology, EMBO Rep 10(3), 201-204; dx.doi.org/10.1038/embor.2009.15.

Doudna, J. A. und Charpentier, E., 2014, The new frontier of genome engineering with CRISPR-Cas9, Science 346(6213), 1077.

ETC Group, 2010, The New Biomassters: Synthetic Biology and the Next Assault on Biodiversity and Livelihoods, Montreal: ETC Group; etcgroup.org/sites/www.etcgroup.org/files/biomassters_27feb2011.pdf.

Gómez-Tatay, L., \& Hernández-Andreu, J. M., 2019, Biosafety and biosecurity in Synthetic Biology: A review. Critical Reviews in Environmental Science and Technology, 49(17), 1587-1621. doi:10.1080/10643389.2019.1579628

Juhas, M., 2016, On the road to synthetic life: the minimal cell and genomescale engineering, Critical Reviews in Biotechnology 36(3), 416-423.

Lander, E., Baylis, F., Zhang, F., Charpentier, E. und Berg, P., 2019, Adopt a moratorium on heritable genome editing, Nature 567(7747), 165-168.

Mackenzie, A., 2010, Design in synthetic biology, BioSocieties 5(2), 180-198.

Pühler, A. et al. (Hg.), 2011, Synthetische Biologie. Die Geburt einer neuen Technikwissenschaft, Berlin, Heidelberg: Springer

Purnick, P. E. M., Weiss, R., 2009, The second wave of synthetic biology: from modules to systems, Nature Reviews Molecular Cell Biology 10(6), 410-422.

3 nature.com/articles/d41586-018-05814-6. 
Regalado, A., 2019, Three years ago an unknown Chinese scientist edited the DNA of human embryos. It was a step on an inexorable path to designer babies, Mit Technology Review 122(1), 63-69.

Sauter, A., Albrecht, S., Doren, D. v., König, H., Reiß, T. und Trojok, R., 2015, Synthetische Biologie - Die nächste Stufe der Bio-und Gentechnologie: Büro für Technikfolgen-Abschätzung beim Deutschen Bundestag (TAB). 


\section{Digitale Erinnerung}

Unter dem Stichwort Lifelogging wird seit den 1980er-Jahren mit Kameras experimentiert, die um den Hals getragen oder in eine Brille integriert sind. Sie zeichnen den persönlichen Alltag einer Person in automatisierten Einzelbildern auf, z. B. alle 30 Sekunden; auch Filmen und Livestreamen des Erlebten wurde mittlerweile möglich. ${ }^{1}$ Damit werden viele Details abrufbar, die in der menschlichen Erinnerung verloren gehen würden. Zusätzlich zum Bild können auch individuelle physiologische Daten wie Puls oder Blutdruck mit aufgezeichnet werden. Die Miniaturisierung von Kameras, Akkus, Speichermedien und Sensoren sowie die fortschreitende Entwicklung und Verfügbarkeit des Internets hat die Träume von frühen Lifeloggern wahr werden lassen (Metalinsen; Gesundheitsdatenmonitoring; Sensorrevolution). Klinische Anwendungen, etwa zur Unterstützung der Erinnerungsleistung von AlzheimerpatientInnen, werden erforscht. Auch generell wird an lifelogging als Unterstützungstechnologie für alternde Gesellschaften geforscht (Climent-Perez et al. 2020), beispielsweise zur Prävention im Gesundheitsbereich (Scherenberg 2019). Technisch problematisch sind noch immer die dabei anfallenden großen Datenmengen. Auch wenn fast ständig verfügbare Cloudspeicher das Speicherproblem teilweise zu lösen scheinen, ist vor allem das gezielte Durchsuchen nach konkreten Inhalten noch immer ein ungelöstes Problem. ${ }^{2}$ Big-Data-Anwendungen, künstliche Intelligenz und Gesichtserkennung sollen hier Abhilfe schaffen. Eine vermehrte Anwendung von Lifelogging kann gesellschaftliche Probleme nach sich ziehen: Die ubiquitäre Aufzeichnung von Bild- und anderen Daten kann zur Überwachung missbraucht werden und kann die Rechte Dritter in Bezug auf Anonymität und Datenschutz verletzen ${ }^{3}$ [Verweis: 10_Ende Anonymität]. Auch psychische und soziale Folgen sind bei einer breitenwirksamen Anwendung zu erwarten (Selke 2020): Wie verändert sich das Verhalten des Einzelnen und die Gesellschaft, wenn jeder Moment des Lebens erfasst und gespeichert wird.

\section{Zitierte Quellen}

Climent-Pérez, P., Spinsante, S., Mihailidis, A., Florez-Revuelta, F. (2020) A review on video-based active and assisted living technologies for automated lifelogging, Expert Systems with Applications, Volume 139, 112847, doi.org/10.1016/j.eswa.2019.112847.

\footnotetext{
1 spectacles.com/at/.

2 technologyreview.com/s/602306/technologists-wont-give-up-on-the-dream-ofmemory-augmentation/.

3 enisa.europa.eu/publications/to-log-or-not-to-log-risks-and-benefits-of-emerginglife-logging-applications/at_download/fullReport.
} 
Scherenberg V. (2019) Prävention via Lifelogging - Möglichkeiten und Grenzen der digitalen Selbstvermessung. In: Pfannstiel M., Da-Cruz P., Mehlich H. (eds) Digitale Transformation von Dienstleistungen im Gesundheitswesen VI. Springer Gabler, Wiesbaden. doi.org/10.1007/978-3-658-25461-2_24.

Selke S. (2020) Lifelogging. In: Friese H., Nolden M., Rebane G., Schreiter M. (eds) Handbuch Soziale Praktiken und Digitale Altagswelten. Springer VS, Wiesbaden. doi.org/10.1007/978-3-658-08357-1_41. 


\section{Social [Ro-]Bots: Maschinen als GefährtInnen?}

Schaffen es virtuelle Software-AgentInnen die Meinung der Wählerlnnen in Internetforen und sozialen Plattformen zu beeinflussen? Wie viele Menschen unterhalten sich mit innen ohne zu wissen, dass es sich bei ihrem Gegenüber um eine Maschine handelt? Und welche Konsequenzen haben emotional lernfähige Maschinen für unser Zusammenleben und nicht zuletzt für unsere Vorstellung von demokratischer Entscheidungsfindung?

Soziale Roboter sind (teil-)autonome Maschinen, die nach sozialen Regeln mit Menschen interagieren und mitunter menschlicher Form nachgebildet sind. ${ }^{1}$ Es kann sich dabei um physisch vorhandene oder auch virtuelle Software-Agenten handeln, die auch Gefühle repräsentieren bzw. vortäuschen können. Virtuelle Agentinnen sind unsere ständigen Begleiterinnen (wie z. B. Siri, Alexa oder Tay auf Smartphones). Aber auch physische Roboter werden vermehrt für Unterrichtszwecke (Rubi), für alltägliche Aufgaben im Haushalt oder zur Unterhaltung (Jibo, Nao, Pepper) eingesetzt. Dem Einsatz im Gesundheitswesen, v. a. als Pflege- und Therapieroboter (Zora, Hobbit ${ }^{2}$, Leka), wird hohes Zukunftspotential zugeschrieben.

Warum könnte sich der Einsatz von sozialen Robotern erhöhen? Der demografische Wandel und die daraus folgenden Bedarfe im Gesundheitswesen und in der Pflege, sowie die Digitalisierung der Produktion und maschinen-induzierte Veränderungen in der Arbeitswelt sind starke Treiber für die Integration von sozialen Robotern in die Gesellschaft (Interaktion mit Social Bots statt „Betriebsanleitungen“).

Die Einführung der digitalen GefährtInnen birgt auch Herausforderungen: Wie verändert sich eine Gesellschaft, in der willfährige Sexroboter, wie z. B. Roxxxy oder Samantha, Frauenpositionen ersetzen ${ }^{3}$ Was passiert mit dem emotionalen Potential von Menschen, die sich in ihrem Umgang vermehrt auf Maschinen stützen? Gerade bei Anwendungen, die experimentell mit Menschen z. B. im Medizin- und Gesundheitsbereich getestet werden (Therapieroboter), gilt es die Selbstbestimmung der involvierten Menschen zu gewährleisten.

Beispiele aus der Vergangenheit zeigen, dass soziale Roboter zweckentfremdet werden können: $z$. B. lernte der Social Bot Tay rassistische Äußerungen zu verbreiten (Graff 2016); und Social Bots beeinflussten mit ihrer Diskussion möglicherweise die US-amerikanischen Wahlen (Grech 2017) (siehe dazu Thema "Microtargeting“). Während bereits Werkzeuge entwickelt werden, die dem Missbrauch entgegenwirken sollen (bspw. die Diens-

\footnotetext{
1 Eine deutschsprachige Vorstudie zum Thema mit Schwerpunkt auf SoftwareAnwendungen hat das Büro für Technikfolgen-Abschätzung beim Deutschen Bundestag im Jahr 2017 erstellt, siehe dazu: tab-beimbundestag.de/de/pdf/publikationen/berichte/TAB-Horizon-Scanning-hs003.pdf.

2 hobbit.acin.tuwien.ac.at/about.html.

3 Einen Überblick zur Literatur und zu ethischen Positionen für und wider die Entwicklung von sogenannten Sexrobotern gibt (Döring, Mohseni, \& Walter, 2020).
} 
te Botometer ${ }^{4}$ und botswatch ${ }^{5}$, die darauf abzielen Social Bots zu identifizieren), bleiben hochbrisante Fragen, die gesellschaftlich verhandelt werden müssen, vorerst unbeantwortet.

\section{Zitierte Literatur}

Döring, N., Mohseni, M. R., \& Walter, R. (2020). Design, Use, and Effects of Sex Dolls and Sex Robots: Scoping Review. J Med Internet Res, 22(7), e18551. doi:10.2196/18551

Graff, B., 2016, Rassistischer Chat-Roboter: Mit falschen Werten bombardiert., Süddeutsche Zeitung.

Grech, B., 2017, Social Bots: Ein Wahlkampf der Algorithmen., Die Presse.

4 botometer.iuni.iu.edu/\#!/.

5 botswatch.de. 


\section{Quantenbiologie}

Neue Forschungsansätze erweitern die klassische Quantenphysik um experimentelle Ansätze der Biologie und öffnen das interdisziplinäre Feld für zukünftige, technologische Anwendungen, wie z. B. die künstliche Photosynthese zur Erzeugung von Biotreibstoffen (Lim et al. 2015), den Quantencomputer oder hocheffiziente Solarzellen (Ball 2011). Diese neuen Forschungstrends werden unter dem Begriff Quantenbiologie zusammengefasst. Der Terminus Quantenbiologie eignet sich, um nach außen hin zu signalisieren, dass in biologischen Systemen interessante moderne Physik erforscht werden kann (Von Rauchhaupt 2013).

Bisher konnte die mögliche Relevanz, die Quantum-Phänomene für die Funktionsweise von lebenden Organismen haben, nur anhand von theoretischen Experimenten und Computersimulationen erforscht werden. Jetzt wurden Quantum-Effekte in bestimmten biologischen Systemen (Lichtsammelkomplexen) bereits experimentell nachgewiesen; das Ausmaß ihrer Wirkung ist jedoch noch unerforscht (Cao et al. 2020).

Wie Geruch wahrgenommen wird oder wie sich Lebewesen (z. B. Zugvögel) anhand von Magnetfeldern orientieren, könnte mit Hilfe der Quantenbiologie geklärt werden. Erste experimentelle Nachweise mit Fruchtfliegen bestätigen die Sinnhaftigkeit der interdisziplinären Forschungsrichtung (Franco et al. 2011; Ritz et al. 2010). Die Erkenntnisse aus dem Feld der Quantenbiologie könnten generell ein verbessertes Verständnis von biologischen Systemen und darüber hinaus die Entwicklung effizienter und robuster Quanten-Technologien für z. B. Solarenergie, Kommunikation und Navigation ermöglichen (Caruso 2016).

Die Quantenbiologie ist einerseits für die Weiterentwicklung der Physik (Quantencomputer), andererseits für Anwendungen (Energiespeicher) und auch Nachahmung biologischer Prozesse (z. B. effiziente Energiegewinnung aus Sonnenlicht) relevant. Die Verknüpfung der beiden Forschungsfelder Quantenphysik und Biologie ${ }^{1}$ und die zukünftige Grundlagenforschung im neuen, interdisziplinären Bereich der Quantenbiologie ist möglicherweise für Österreich, das einen hohen Kompetenzgrad in der Quantenforschung aufweist, interessant.

1 iqoqi-vienna.at/en/research/zeilinger-group/quantum-science-and-application-inbiology/. 


\section{Zitierte Quellen}

Ball, P., 2011, Physics of life: The dawn of quantum biology, Nature News 474(7351), 272-274.

Cao, J., Cogdell, R. J., Coker, D. F., Duan, H.-G., Hauer, J., Kleinekathöfer, U., Jansen, T. L. C., Mančal, T., Miller, R. J. D., Ogilvie, J. P., Prokhorenko, V. I., Renger, T., Tan, H.-S., Tempelaar, R., Thorwart, M., Thyrhaug, E., Westenhoff, S. und Zigmantas, D., 2020, Quantum biology revisited, Science Advances 6(14), eaaz4888 advances.sciencemag.org/content/6/14/eaaz4888.abstract.

Caruso, F., 2016, What is Quantum Biology? Council for the Lindau Nobel Laureate Meetings e.V.; lindau-nobel.org/what-is-quantum-biology/

Franco, M. I., Turin, L., Mershin, A. und Skoulakis, E. M. C., 2011, Molecular vibration-sensing component in Drosophila melanogaster olfaction, Proceedings of the National Academy of Sciences 108(9), 3797-3802; pnas.org/content/108/9/3797.abstract.

Lim, J. et al., 2015, Vibronic origin of long-lived coherence in an artificial molecular light harvester, Nature communications 6, 7755.

Rinaldi, A., 2012, When life gets physical, Quantum effects in selected biological systems have been confirmed experimentally, but how widespread is their role remains unclear 13(1), 24-27; embor.embopress.org/content/embor/13/1/24.full.pdf.

Ritz, T., Yoshii, T., Helfrich-Foerster, C. und Ahmad, M., 2010, Cryptochrome: A photoreceptor with the properties of a magnetoreceptor?, Communicative \& Integrative Biology 3(1), 24-27; ncbi.nlm.nih.gov/pmc/articles/PMC2881235/.

Von Rauchhaupt, U., 2013, Quantenbiologie: Das Leben ist ein Quantenspiel, Frankfurter Allgemeine Zeitung; faz.net/-gx5-75vfq. 


\section{Digitales Lernen: Offene Infrastrukturen für Bildungsgerechtigkeit}

\section{Zusammenfassung}

Digitale Lerntechnologien ermöglichen es - gerade in Krisenzeiten -, den Unterricht und die Bildung aus der Ferne fortzusetzen und den Zugang zu Bildungsangeboten in Anbetracht der digitalen Transformation der Gesellschaft langfristig zu garantieren. Gleichzeitig bieten digitale Innovationen im Bildungswesen große Chancen und Möglichkeiten, durch die Sammlung und Analyse von Bildungsdaten Erkenntnisse über die Entwicklungen des Lehrens und Lernens zu gewinnen und individuelle Lernprozesse zu unterstützen. Welche Voraussetzungen für die transparente und angemessene Nutzung von Online-Tools und zukünftigen Softwarearchitekturen notwendig sind und wie eine nachhaltige, europäische Lerninfrastruktur, die auf eine Verringerung der sozialen Kluft abzielt, vorangebracht werden könnte, wird nachstehend beleuchtet.

\section{Überblick zum Thema}

Die soziale Ungleichheit in der österreichischen Gesellschaft ist in der Corona-Krise gestiegen, betroffen sind besonders Menschen mit geringer formaler Bildung. ${ }^{1}$ Gerade in Krisenzeiten, in denen die Ansammlung von Menschen als problematisch in Hinblick auf die Krankheitsverbreitung gesehen wird und Schulen, Universitäten und Bildungseinrichtungen über mehrere Monate hinweg geschlossen bleiben, tritt das Potenzial digitaler Innovationen, wie z.B. E-Learning und digitale Verwaltung im Bildungswesen, besonders hervor. Ministerien und Landesverwaltungen setzen - unter dem Schlagwort „Distance Learning“ (Fernlehre) - zunehmend auf den Einsatz von digitalen Tools und Softwarelösungen, um den virtuellen Handlungsraum zu erweitern und Lernen sowie sozialen Austausch auf Distanz zu ermöglichen. ${ }^{2}$ Das Angebot digitaler Innovationen im Bildungswesen ist vielfältig: Es zielt auf die Bereitstellung von Lern- und Bildungsinhalten in Form von virtuellen Wissensbeständen und Datenbanken, auf die Unterstützung von Lern- und Unterrichtsprozessen anhand von Web-Anwendungen (Apps), auf die digitale Verwaltung von Bildungseinrichtungen mit Hilfe spezieller Softwarelösungen und umfassen sowohl offene als auch proprietäre Software-Angebote für den Unterricht, wie Lernplattformen ${ }^{3}$,

Vielfältiges Angebot digitaler Innovationen im Bildungswesen

1 Das zeigt eine aktuelle Panelumfrage zur Corona-Krise der Universität Wien, siehe viecer.univie.ac.at/coronapanel/.

2 bmbwf.gv.at/Themen/schule/beratung/corona/corona_fl/dlsp.html; tirol.gv.at/gesellschaft-soziales/familie/foerderungen/tiroler-digi-scheck/.

3 So zum Beispiel die weltweit eingesetzte Open-Source-Plattform Moodle, ein freies objektorientiertes Kursmanagementsystem und eine Lernplattform, die kooperativer Lehr- und Lernmethoden unterstützt moodle.org/?lang=de; aber auch proprietäre Angebote wie z.B. LessonUp lessonup.com/en; ShadowPuppet getpuppet.co; NearPod nearpod.com. 


\author{
Öffentliche und \\ kostenfreie Angebote für \\ Bildungszwecke
}

Proprietäre

Webanwendungen und Software-Lösungen

Learning Analytics, Academic Analytics und Educational Data Mining
Präsentations- und Visualisierungswerkzeuge ${ }^{4}$ oder Social-Media-Tools. ${ }^{5}$ Darüber hinaus unterstützen Übersetzungs-Apps ${ }^{6}$ das Erlernen von Sprachen und die Kommunikation über Sprachbarrieren hinweg.

Neben vielzähligen kommerziellen Angeboten, entstehen zunehmend öffentliche Angebote, wie z.B. die Bildungsplattform edutube ${ }^{7}$, die in Anlehnung an die weltweit bekannte Plattform Youtube journalistisch verlässlich recherchierte Kurzvideos und Dokumentationen zur Verfügung stellt und vor allem auf die nicht-kommerzielle Nutzung zu Bildungszwecken ausgerichtet ist. Das europäische Kulturarchiv Europeana ${ }^{8}$ stellt eine digitale Sammlung von über 50 Millionen Objekten (Bücher, Musik, Kunstwerke, u.v.m.) zur Verfügung und adressiert in einem ausgewählten Abschnitt Lehrkräfte mit seinen Bildungsinhalten. Darüber hinaus gibt es eine breite Palette an digitalen Schulbüchern ${ }^{9}$, die von den Schulbuchverlagen online - und in Krisenzeiten oft auch gratis - angeboten werden. Zur Unterstützung des (Fern-)Unterrichts kommen bereits Open-Source-Lösungen, d.h. freie Software mit der Möglichkeit zur selbständigen Verwaltung und organisational angepassten Einrichtung, zum Einsatz. ${ }^{10}$

Im Bereich der Schulverwaltung zeigt sich ein anderes Bild: hier werden proprietäre Webanwendungen, wie bspw. edu.FLOW ${ }^{11}$ (zur Erstellung elektronischer Formulare und zur strukturierten Kommunikation zwischen bspw. Klassenvorständen und SchülerInnen oder zwischen LehrerInnen und Schuladministration), Untis/WebUntis ${ }^{12}$ (elektronisches Klassenbuch, elektronische Hausaufgabenverwaltung) oder SOKRATES ${ }^{13}$ (Schulverwaltung) eingesetzt. ${ }^{14}$ Auch an Universitäten ist der Einsatz von proprietären Software-Lösungen zur Bereitstellung von E-Learning-Angeboten und zur Kommunikation zwischen Lehrkörper und Studierenden beobachtbar $^{15}$; zunehmend werden aber auch Open-Source-Systeme ${ }^{16}$ eingerichtet und weiterentwickelt.

Mit dem vermehrten Einsatz digitaler Anwendungen im Bildungsbereich ergeben sich neue Möglichkeiten, Daten im Bildungssektor zu sammeln und zu analysieren. Wissenschaftliche Unterfangen im Bereich „Learning

4 Z.B. Sutori sutori.com; Prezi prezi.com/de/.

5 Z.B. SchoolFox schoolfox.com oder SchoolUpdate schoolupdate.com.

6 Z.B. uugot.it uugot.it oder DeepL deepl.com/translator.

7 edutube.at.

8 europeana.eu/de.

9 bmbwf.gv.at/Themen/schule/beratung/corona/corona_fl/dsb.html.

10 Siehe bspw. desktop4education d4e.at/.

11 edusuite.at/edu-flow-3/.

12 untis.at.

13 bitmedia.at/sokrates-schulverwaltung/.

$14 \mathrm{Zu}$ beobachten ist auch der zunehmende Einsatz von Google-Anwendungen an Schulen im Zuge der Coronakrise, siehe epicenter.works/content/google-aufempfehlung-des-ministeriums-massiv-im-einsatz-an-oesterreichischen-schulen.

15 Z.B. Adobe Connect, GoogleEdu, MS Teams, WebEx oder Zoom.

16 Z.B. Big Blue Button an der Universität Graz bigbluebutton.org oder Moodle an der Universität Wien zid.univie.ac.at/e-learning/. 
Analytics“ (Lernanalyse) nutzen Bildungsdaten (sogenannte "intelligente Daten", d.h. vom Lernenden produzierte Daten und Analysemodelle), um Informationen zu gewinnen und soziale Zusammenhänge zu entdecken oder auch um Lernprognosen zu erstellen und daraus Empfehlungen abzuleiten (Larrabee Sønderlund et al. 2019; Markus/Martin 2018). Eine im Entstehen begriffene Disziplin, die damit in Zusammenhang steht, ist „Educational Data Mining“. ${ }^{17}$ Sie setzt sich mit der Entwicklung von Methoden zur Erforschung der immer umfangreicheren Daten aus dem Bildungsbereich auseinander, mit dem Ziel, diese Methoden zum besseren Verständnis der SchülerInnen und des Umfelds, in dem sie lernen, einzusetzen. Unter dem verwandten Begriff „Academic Analytics“ werden ähnliche Unterfangen verstanden, die auf Bildungsdaten aus dem Hochschulsektor abzielen.

Die vielfältigen, wissenschaftlichen Ansätze der Datenanalyse zeigen, dass Bildungsdaten eine wertvolle Ressource sind, die zur Bearbeitung vielfältiger Problemstellungen und Fragen geeignet sind. Sie könnten zukünftig vor allem in Hinblick auf die digitale Kluft in der Gesellschaft eine wichtige Grundlage für das Monitoring von Entwicklungen im Bildungsbereich sein und darüber hinaus politische Entscheidungsfindung unterstützen (Broos et al. 2020). Voraussetzung dafür ist der Zugang zu den Bildungsdaten. Werden diese über proprietäre Cloud-Anwendungen generiert und analysiert, sind sie nicht oder nur begrenzt für die Bildungsforschung und Bildungspolitik zugänglich. Wenn im Bildungswesen dagegen umfassende, kollaborative Bildungsplattformen weiterentwickelt werden, können Daten entsprechende der DSGVO für Analysezwecke und im Sinne der dafür eingesetzten Steuermittel öffentlich zugänglich gemacht werden. Bereits in der Vergangenheit wurden Fragen aufgeworfen, die die Art und Weise der Bereitstellung digitaler Infrastruktur für vielfältige Zwecke und Einsatzbereiche im Bildungswesen in den Mittelpunkt rücken. ${ }^{18}$ Die transparente Auswahl geeigneter Hard- und Software ist dabei zentral, v.a. um Lock-in-Effekte und den Verlust von Nutzungsrechten an Daten zu vermeiden.

\section{Relevanz des Themas für das Parlament und für Österreich}

Im gegenwärtigen globalen Trend einer immer umfassenderen PlattformÖkonomie haben wenige private Großkonzerne umfassenden Zugang zur Ressource „Daten“ (siehe Thema „Kollaborative Plattformen“). Wenn der Bildungssektor konkurrenzfähige Plattformen für Bildungsangebote und Bildungsdatenanalyse bereitstellen soll, stellt sich die Frage, wie digitale Infrastrukturen im Bildungswesen so gestaltet werden können, dass auch
Daten im Bildungssektor als wertvolle Ressource

\section{Besondere \\ Anforderungen an \\ digitale Infrastrukturen \\ im Bildungswesen}

17 Ein Überblick zu Fragenstellungen und Themen im Bereich, kann in den Conference-Proceedings der 10. Konferenz zu Educational Data Mining in Wuhan 2017 eingesehen werden: educationaldatamining.org/EDM2017/proc_files/proceedings.pdf.

18 Siehe Diskussion um die Beschaffung der Schulverwaltungs-Software SOKRATES: derstandard.at/story/2000019259030/sokrates-laesst-schulen-uebersoftware-gruebeln 
in Zukunft Steuerungsfähigkeit und demokratische Kontrolle gegeben sind. In Hinblick auf die Proklamation zur europäischen Säule der sozialen Rechte, ${ }^{19}$ die jedem Menschen das Recht auf eine qualitativ hochwertige und integrative Bildung, Ausbildung und lebenslanges Lernen zuspricht, nimmt Österreich als Mitgliedsstaat und in seiner Verantwortung für Bildung, Ausbildung und Kulturpolitik eine wichtige Rolle ein. Um die europäische Zielsetzung der Etablierung eines gemeinsamen Bildungsraumes bis 2025 zu erreichen, braucht es auf nationaler bzw. auch auf lokaler und regionaler Ebene Initiativen aus dem Bildungssektor für den Bildungssektor.

\section{Vorschlag weiteres Vorgehen}

Ein Foresight-Prozess, der auf die partizipative Identifikation von zukünftigen Anforderungen und Erwartungen an digitale Systeme und Lerndatenanalyse im Bildungssektor abzielt sowie einen breiten Austausch zu Errichtungsmöglichkeiten unabhängiger Bildungsinfrastruktur und offenen Lernplattformen anregt, könnte dazu beitragen, der sozialen Kluft entgegenzuwirken. Darüber hinaus könnten Fragen der Datenhoheit und Datennutzung ausgelotet werden: Wie könnten Aufträge für die Errichtung von Infrastruktur öffentlich und transparent implementiert bzw. vergeben werden, ohne mit der Verpflichtung gegenüber einzelnen Infrastrukturanbietern verzerrende Marktverhältnisse zu schaffen (Lock-in-Effekte)? Wie könnten Bildungsdaten genutzt werden, um die soziale Kluft zu verringern und um zur Etablierung eines europäischen Bildungsraums beizutragen? Welche Risiken könnten durch die datenbasierte Vorhersage von Lernverhalten und den daraus abgeleiteten Politikmaßnahmen entstehen? Welche Voraussetzungen müssen gegeben sein, um die Privatsphäre von Lernenden und Lehrenden zu schützen und einen sensiblen Umgang mit personenbezogenen Daten bei der Analyse von Lernverhalten pflegen zu können?

\section{Zitierte Literatur}

Broos, T. et al., 2020, Coordinating learning analytics policymaking and implementation at scale, British Journal of Educational Technology $n / a(n / a)$.

Larrabee Sønderlund, A., Hughes, E. und Smith, J., 2019, The efficacy of learning analytics interventions in higher education: A systematic review, British Journal of Educational Technology 50(5), 2594-2618.

Markus, E. und Martin, E., 2018, Learning Analytics an Schulen - Hintergrund und Beispiele, Medienimpulse 56(1).

19 data.consilium.europa.eu/doc/document/ST-13129-2017-INIT/de/pdf. 


\section{Dezentrales KI-Lernen: Gesellschaft als Reallabor?}

Die Erforschung Künstlicher Intelligenz (KI) prägt bereits unseren Alltag: die Eingabehilfe einer Handytastatur, Algorithmen zur Spracherkennung und für Musikempfehlungen, Fotoauswahl und Gesichtserkennung sind Beispiele für KI-Anwendungen, die ständig weiterentwickelt und praktisch getestet werden. Künstliche Intelligenz hat wesentlich zum Erfolg großer IT-Unternehmen beigetragen, da diese in der Lage sind, große Mengen an Nutzungsdaten zu sammeln und zentral zu verarbeiten. In der bisherigen Erfahrung führte die KI-Entwicklung zu einer Winner-takes-it-allDynamik: umso umfangreicher die Trainingsdaten einer KI, desto treffender die Prognosen, desto effektiver die angebotene KI-Dienstleistung. ${ }^{1}$ Prinzipiell braucht KI-Lernen große Rechenkapazitäten, weshalb schon bisher Cloud-Lösungen für KI-Lernen genutzt wurden (AWS, Microsoft, Google). ${ }^{2}$ Diese Cloud-Lösungen bauen auf einer zentralisierten Architektur auf: Nutzungsdaten werden zentral in der Cloud gespeichert und dann zum Training von Algorithmen genutzt. Die Nachteile einer zentralen Datenspeicherung bestehen darin, dass große Datenmengen transferiert werden müssen, was Kosten verursacht; Latenzprobleme verhindern oft die erforderliche Echtzeit-Inferenz; Verschwiegenheitsanforderungen in Bereichen mit sensibler Datenverarbeitung verhindern die CloudSpeicherung; Datenübertragung zu entlegenen Cloud-Servern bietet Einfallstore für Hacker-Angriffe (siehe Thema "Cloud Computing").

Dezentrale Ansätze im Maschinenlernen versuchen diesen Nachteilen entgegenzuwirken und werden in Anbetracht der zunehmenden Vernetzung von Geräten und damit steigender, dezentraler Rechnerleistung immer attraktiver. Einer dieser Ansätze ist „Edge Computing“. Die Idee ist, dass Informationsverarbeitung am Rand des Netzwerks („Edge“), d.h. z.B. direkt am Endgerät stattfindet, um einerseits von den Vorteilen einer Cloud zu profitieren und andererseits Verzögerungen bei der Datenübertragung zu vermeiden. Einen anderen Fokus legt „Federated Learning“, eine neue Trainingsmethode für $\mathrm{KI}$, die auf kooperatives Lernen anhand eines geteilten Vorhersagemodells abzielt. Anders als bisher ist dazu kein zentraler Datensatz notwendig, sondern die Verbesserungen und Lernprozesse der einzelnen, mobilen Geräte werden als Update verschlüsselt an eine Cloud geschickt. In der Cloud werden die gesammelten Updates aller NutzerInnen zusammengeführt und helfen, das zentrale Trainingsmodell zu verbessern. Das verbesserte Modell wird dann wiederrum auf die mobilen Geräte zurückgespielt und kann gleich genutzt werden. Die Vorteile dieser Verarbeitungsweise liegen im reduzierten Datenvolumen der Updates im Vergleich zur Übertragung der gesamten Nutzungsdaten und damit geringeren Kosten. Zudem bietet Federated Learning einen

\footnotetext{
1 mattturck.com/the-power-of-data-network-effects/.

2 hackernoon.com/federated-learning-a-step-closer-towards-confidential-ai$7 \mathrm{ac} 4 a f a 9 b 437$.
} 
höheren Datenschutz, da alle persönlichen Daten am mobilen Gerät verbleiben und lediglich die Updates des Modells verschlüsselt an die Cloud übermittelt werden.

Gerade für Bereiche, in denen es aufgrund von sektoralen Geheimhaltungsbestimmungen oder Geschäfts- und Sicherheitsinteressen nicht gewünscht ist, Daten an Dritte weiterzugeben (Bankenwesen, Gesundheitsbereich, Versicherungen, Militär), eröffnet dezentrales Maschinenlernen neue Zukunftsoptionen. Die Dezentralisierung von digitaler Infrastruktur für KI-Lernen wird auch in Zukunft die umfassende Marktmacht großer Technologiekonzerne nicht mindern. Die Möglichkeiten von Federated Learning könnten aber zum vermehrten Eintritt neuer Akteure in den Markt für KI-Lernen führen, z.B. Start-ups in Nischenbereichen, wie z.B. Krebsforschung. ${ }^{3}$ Durch einen offeneren Zugang zur KI-Infrastruktur und durch dezentrale KI-Lernmethoden könnten die Nutzung und Verbesserung von $\mathrm{KI}$ zu Effizienzgewinnen in prognostischen Anwendungen führen und damit gesellschaftliche Vorteile realisieren. Trotz vielversprechender Visionen, bestehen auch Zweifel an der neuen Technologie: KritikerInnen argumentieren, dass das geteilte, globale Prognosemodell durch jedeN Teilnehmerln von Federated Learning manipuliert werden kann (Bagdasaryan et al. 2019).

Gerade bei der KI-Entwicklung wird deutlich, dass Forschungsprozesse und die mit innen verbundenen Risiken über institutionalisierte Grenzen der Wissenschaft hinaus- und in die Gesellschaft hineingetragen werden, d.h. Gesellschaft als Reallabor für experimentelle Forschung genutzt wird (Krohn/Weyer 1989). Ob dezentrale KI-Technologien begrenzen können, dass die Gesellschaft wie bisher als Experimentierfeld von Facebook, Amazon und Google dient, bleibt offen. Welche sozialen, ethischen und politischen Implikationen die Anwendung von Federated Learning für die Verarbeitung personenbezogener Daten hat, könnte weiterführend erarbeitet werden.

\section{Zitierte Quellen}

Bagdasaryan, E., Veit, A., Hua, Y., Estrin, D. und Shmatikov, V., 2019, How To Backdoor Federated Learning, arxiv.org/pdf/1807.00459.pdf.

Krohn, W. und Weyer, J., 1989, Gesellschaft als Labor: Die Erzeugung sozialer Risiken durch experimentelle Forschung, Soziale Welt 40(3), 349-373.

\footnotetext{
3 owkin.com.
} 


\section{Open Access - jetzt aber wirklich?}

Die Erforschung Künstlicher Intelligenz $(\mathrm{KI})$ prägt bereits unseren Alltag: Open Access (OA) steht für den unbeschränkten und kostenlosen Zugang zu wissenschaftlichen Publikationen und Daten im Internet. Damit ist der Anspruch verbunden, dass die Ergebnisse öffentlich finanzierter Forschung auch für alle Interessierten öffentlich zugänglich sein sollten. Das Ziel von OA wird bereits seit vielen Jahren verfolgt, es handelt sich jedoch um einen langwierigen Umstellungsprozess des wissenschaftlichen Publikationssystems mit dem Ziel, dass nicht die LeserInnen (bzw. ihre Institutionen, Bibliotheken), sondern die Autorlnnen (bzw. deren Institutionen) oder wissenschaftliche Gesellschaften und Konsortien für die Publikationskosten aufkommen. Insbesondere die großen, internationalen wissenschaftlichen Verlage verdienen am bisherigen System durch hohe Abonnementpreise bzw. am Übergangssystem der „Hybridzeitschriften“ durch hohe Preise für das Open-Access-Stellen („Freikaufen“) einzelner Artikel. Diese Verlage sind daher nur zögerlich oder gar nicht bereit, auf OA umzustellen. Da sich die Alternative, die Artikel individuell nach Veröffentlichung in sogenannte OA-Repositorien hochzuladen als lückenhaft und aufgrund der Vertragsgestaltung der Verlage oft als unmöglich herausgestellt hat, versuchen die öffentlichen Forschungsförderer seit längerem den Prozess dadurch zu beschleunigen, dass sie OA zu einer Verpflichtung für die wissenschaftlichen AutorInnen machen. Die dabei entstehenden zusätzlichen Kosten (für das Freikaufen im Hybridmodell oder durch die AutorInnengebühren bei reinen OA-Journalen) werden derzeit entweder von den Institutionen der AutorInnen oder von den Fördereinrichtungen selbst getragen. ${ }^{1}$

Die jüngste Initiative ging am 4. September 2018 unter dem Namen „COAlition Plan S" von Science Europe, dem Dachverband der europäischen Forschungsförderorganisation aus. ${ }^{2}$ Der Plan wird von der EUKommission und dem European Research Council (ERC) unterstützt, in Österreich ist der FWF federführend. Die finale Fassung wurde im Mai 2019 publiziert $^{3}$ und setzt als ambitioniertes Ziel, dass ab dem 1. Jänner 2021 jedwede mit öffentlichen Mitteln geförderte Forschung OA publiziert sein muss. Der Plan formuliert zehn Prinzipien. Unter anderem wird das sog. Hybridmodell abgelehnt, es müssen also Zeitschriften (und mit gewisser Verzögerung auch Bücher) sein, die vollständig OA erscheinen, nicht nur einzelne, freigekaufte Artikel. Wenn entsprechende Plattformen oder Journale noch nicht existieren, weil die bisherigen Verlage nicht bereit sind, diese im jeweiligen Fach anzubieten, sollen mit Hilfe öffentlicher Gelder solche rasch aufgebaut werden. Die Umsetzung wird also kurzfris-

\footnotetext{
1 Allgemein zu Open Access siehe oana.at/ueber-open-science/open-accessressourcen/ bzw. open-access.net/AT-DE/informationen-zu-open-access/.

2 scienceeurope.org/coalition-s/.

3 coalition-s.org.
} 
tig zusätzlich Geld kosten, mittelfristig werden jedoch die hohen Abonnementkosten stark zurückgehen. Zugleich wird der Kreis der NutzerInnen viel größer, da die wissenschaftlichen Ergebnisse fortan nicht mehr nur in spezialisierten Bibliotheken zugänglich sein werden.

Österreich könnte einen maßgeblichen Beitrag leisten, um den Übergang rasch zu bewerkstelligen. Neben dem FWF, der bereits erheblich Open Access in seinem Bereich gefördert hat, und dem WWTF müssten auch andere Forschungsförderer wie die FFG mit den von ihr verwalteten Programmen, die ÖAW mit ihren Stipendien und Preisen, der Jubiläumsfonds der Nationalbank, die Forschungsförderaktivitäten auf Länderebene usw. mitmachen, um Plan S tatsächlich österreichweit in die Tat umzusetzen.

Dass sich Österreich an dieser internationalen Entwicklung aktiv beteiligen will, ist auf politischer Ebene mittlerweile geklärt: Das Regierungsprogramm 2020-2024 enthält ein klares Bekenntnis zu Open Access: „Die Bundesregierung unterstützt aktiv den Plan S zur Implementierung von Open Access. In weiterer Folge sollen die Prinzipien des Plan S auch von allen Hochschulen und außeruniversitären Forschungseinrichtungen in Österreich umgesetzt werden."4 Nun bedarf es konkreter ökonomischer und wissenschaftspolitischer Umsetzungsmaßnahmen.

4 Verantwortung für Österreich. Regierungsprogramm 2020-2024, S. 305, online abrufbar z.B. hier: gruene.at/themen/demokratie-

verfassung/regierungsuebereinkommen-tuerkisgruen/regierungsuebereinkommen.pdf. 


\section{Zukunft der Quantentechnologie: Quantencyberkrieg oder leistbares Quanteninternet für Alle?}

\section{Zusammenfassung}

In den letzten Jahren hat der globale Wettlauf um die Entwicklung quantentechnologischer Anwendungen, nicht zuletzt durch beachtliche öffentliche Investitionen, neuen Aufschwung erhalten. Nationalstaaten und ihre hochrangigen Forschungseinrichtungen (Akademien der Wissenschaft, Technologiezentren, Innovationsagenturen, u.Ä.) fördern die Nutzbarmachung und Markteinführung von Quantentechnologie und mittlerweile kündigen auch Unternehmen an, in dem Bereich Durchbrüche zu erzielen. Was begrifflich als homogenes Technologiefeld erscheint, ist von unterschiedlichen Paradigmen geprägt. ${ }^{1}$ Die Zukunftsversprechen reichen vom Quantencomputer, der spezielle Aufgaben um Größenordnungen schneller lösen kann als ein klassischer Computer, über theoretisch absolut abhörsichere Quantenkommunikation, bis hin zum globalen Quanteninternet, das über Satelliten die Welt vernetzen soll. Doch was sind die Voraussetzungen für eine verantwortungsvolle Nutzung von Quantentechnologien in der Zukunft? Schon jetzt gehen mit den vielversprechenden Quantenzukünften dystopische Vorstellungen einher: allein die notwendige Großinfrastruktur (z.B. Satelliten) hat exklusive Zugänge zur Technologie zur Folge und lässt ein Szenario umfassender Kontrolle und Überwachung von digitalem Leben realistisch erscheinen. ${ }^{2}$ Gerade in Hinblick auf das forschungspolitische Ziel, die Marktreife von Quantentechnologien zu erreichen, ist die Antizipation ethischer, rechtlicher und sozialer Aspekte zur Schaffung von Rahmenbedingungen unabdingbar.

\section{Überblick zum Thema}

Quantentechnologien versprechen Antworten auf Problemstellungen, die derzeit mit keiner anderen Technologie gelöst werden können. Trotz der hohen Erwartungen an zukünftige technische Anwendungen sind Quantentechnologien nach wie vor in weiten Bereichen ein Feld der Grundlagenforschung. Aufgrund der vielfältigen Anwendungsmöglichkeiten und Einsatzgebiete für Quantentechnologie ist wenig transparent, was ein Quantensystem ausmacht. Sinnvoll ist die Definition, dass ein System dann ein Quantensystem ist, wenn seine Eigenschaften von der Quantenphysik dominiert sind (Elser et al. o.J.).

\footnotetext{
1 Neben Quantenphysik oder Quanteninformatik, trägt ebenso Wissen aus dem Ingenieurwesen und aus sozialwissenschaftlichen Disziplinen, wie z.B. Bildungswissenschaften, zu quantentechnologischer Forschung bei.

2 Auch der Global Risks Report beschreibt ein ähnlich dystopisches Szenario in dem hochleistungsfähige Quantencomputer als disruptives Zukunftsereignis auftreten, das zu einem weitreichenden Kollaps des digitalen Lebens und zum Verlust von Geheimnissen, sensiblen persönlichen und vertraulichen Unternehmens- und Staatsdaten durch wegfallende Sicherheitsgarantien führt; siehe World Economic Forum (2019).
}

Nach wie vor in weiten Bereichen ein Feld der Grundlagenforschung 
'Quanten-Superposition

„No-Cloning"-Prinzip

Quantenphänomen der Verschränkung

Quantenkryptographie und Quantenschlüsselverteilung
Es gibt mehrere für die Quantentechnologien wesentliche quantenphysikalische Prinzipien. Das wohl bekannteste ist das Prinzip der QuantenSuperposition oder Quantenüberlagerung, das auch anhand „Schrödingers Katze" beschrieben werden kann. Es besagt, dass Teilchen gleichzeitig in mehreren Quantenzuständen bzw. an mehreren Orten sein können und erst durch eine Messung wird ein eindeutiger Zustand bzw. Ort festgelegt. Ein weiteres Detail liefert die Heisenbergsche Unschärferelation: sie besagt, dass in der Regel nur Wahrscheinlichkeitsaussagen und keine präzisen Vorhersagen für Messungen an einem einzelnen Quantensystem vorgenommen werden können (Elser et al. o.J.). Auf den ersten Blick mag dies als hinderlich erscheinen, es ist aber gerade diese Eigenschaft, die der Quantenschlüsselverteilung einen Vorteil verschafft: es ist aufgrund physikalischer Gesetze nicht möglich, unbekannte Quantensysteme zu kopieren. Würde nun ein Spion ein „Quantentelefonat“, d.h. Quantenkommunikation zwischen zwei Parteien, abhören und eine Messung vornehmen, könnte man die „Störung“ sofort erkennen. Dieses „NoCloning"-Prinzip ist ausschlaggebend für den Austausch von sogenannten „Schlüsseln“ bei der Quantenschlüsselverteilung (auf Englisch Quantum Key Distribution, QKD). Dazu kommt das Quantenphänomen der Verschränkung (besser bekannt unter dem englischen Begriff „Entanglement“). Obwohl ein einzelnes Quantensystem sehr unscharfe Messresultate liefert, können zwei Quantensysteme perfekt miteinander korreliert sein und dadurch präzise Vorhersagen zu den erwartbaren Messresultaten ermöglichen. Dieses Prinzip ist die Grundlage für den Quantencomputer.

Auch international erfolgen gegenwärtig Aktivitäten, die auf zukünftige Anwendungen orientiert sind: zusammen mit chinesischen Wissenschaftlern haben österreichische Quanten-Experten das erste „Quantentelefonat" über eine Distanz von $7.600 \mathrm{~km}$ durchgeführt. ${ }^{3}$ Google hat dieses Jahr angekündigt, einen Quantencomputer entwickelt zu haben, der in der Lage ist eine spezielle Aufgabe um Größenordnungen schneller zu lösen als ein klassischer Computer (vgl. Arute et al. 2019). ${ }^{4}$

Die Quantenkryptographie und im Speziellen die Quantenschlüsselverteilung ist eine konkrete Anwendung des breiteren Felds der Quantenkommunikation. Sie ist das Teilgebiet der Quantentechnologien, das gegenwärtig das höchste Potenzial zur Kommerzialisierung aufweist. Erste erfolgreiche Demonstrationen von Quantenschlüsselverteilung weltweit wurden bereits Anfang der 2000er im Rahmen des Projekts SECQOC in Österreich durchgeführt (Peev et al. 2009). Auch in den U.S.A. und vor allem in China wird seit einigen Jahren verstärkt an der Umsetzung von QKD Verschlüsselungstechnologie in die Praxis gearbeitet. 2018 stellte

3 oeaw.ac.at/en/detail/news/secure-quantum-communication-over-7600kilometers-2/

4 Dieser Fortschritt ist auch unter dem englischen Schlagwort „Quantum Supremacy“, auf Deutsch „Quantenüberlegenheit“" bekannt. Allerdings ist höchst umstritten, ob es sich tatsächlich um einen Durchbruch handelt, vgl. zeit.de/digital/internet/2019-10/quantencomputer-google-innovationsupercomputer-quantum-supremacy. 
die Europäische Kommission ihre Quantum Flagship Initiative ${ }^{5}$ vor, die für die nächsten zehn Jahre bis zu einer Milliarde Euro für die Forschung an Schlüsselbereichen der Quantentechnologien in Europa bereitstellen wird. ${ }^{6}$ Die am Kick-off Event der Initiative in der Wiener Hofburg vorgestellten Vorhaben sind vielfältig: angefangen von leistbarer Quantenkommunikation für alle, über die Weiterentwicklung eines Quantensimulators zum Aufbau eines Quantenprozessor-Netzwerkes, bis hin zur Entwicklung industrieller Quantencomputer. Grundlegende Idee der Förderinitiative ist es Quantentechnologien mit der langfristigen Vision eines globalen Quanteninternets zur Marktreife zu bringen. Erste Schritte in diese Richtung sind beispielsweise die Verkleinerung von Bauteilen, was langfristig auch die Kosten von Quantengeräten verringern soll, oder die Standardisierung von Quantenelementen, um deren Integration in traditionelle Kommunikationssysteme (Glasfasernetze der Telekomanbieter) zu ermöglichen. Anhand des Beispiels Quantenkryptographie wird deutlich, dass es zusätzlich zur Technologieentwicklung notwendig ist Infrastrukturen zur Schlüsselverteilung ${ }^{7}$ aufzubauen (Glasfasernetze, Satelliten, u.Ä.).

\section{Relevanz des Themas für das Parlament und für Österreich}

Österreich hat eine international führende Rolle in der Quantenforschung. Eine Vielzahl an Einrichtungen forscht an Quantentechnologien: die Universität Wien, die Österreichische Akademie der Wissenschaften (ÖAW), das AIT Austrian Institute of Technology, sowie die Universität Innsbruck, und die Technische Universität Wien. Nicht nur die wegweisenden Experimente im Bereich Quantenphysik wurden in Österreich durchgeführt, sondern auch Hard- und Software für Quantentechnologien wird zurzeit in Österreich entwickelt. Die hohe Beteiligung Österreichs an mehreren Projekten der Quantum Flagship Initiative (z.B. CiViQ ${ }^{8}$, UniQorn ${ }^{9}$ ) ist ein weiterer Indikator für die etablierte Position Österreichs. Doch nicht nur in der Forschung und Entwicklung ist das Potenzial hoch; gerade Quantentechnologien, die sich für militärische Anwendungen eignen sind von hohem internationalem Interesse und könnten auf ein zukünftig, mögliches Technologie-Wettrüsten hindeuten. In Österreich wird gegenwärtig Quantentechnologie ${ }^{10}$ breit gefördert und im Rahmen der Sicherheitsforschung werden spezielle Projekte zu Quantenkryptographie (QKD) mit dem Ziel die Datensicherheit von Behörden zu erhöhen ${ }^{11}$ ausgeschrieben.

\footnotetext{
5 qt.eu/.

6 wienerzeitung.at/nachrichten/wissen/forschung/998839-Quantenforschung-fuerden-Markt.html

7 Auf diesen Bedarf reagierte die Europäische Kommission zeitnah dieses Jahr mit einem neuen Schwerpunktprogramm „Quantenkommunikationsinfrastruktur für Europa“, siehe ec.europa.eu/digital-single-market/en/news/future-quantumeu-countries-plan-ultra-secure-communication-network.

8 civiquantum.eu/.

9 quantum-uniqorn.eu/.

$10 \mathrm{ffg}$.at/quantenforschung-und-technologie.

$11 \mathrm{ffg}$.at/kiras/ausschreibung-2019.
}

Quantum Flagship Initiative

Zukunftsvision: globales Quanteninternet und Quanteninfrastruktur

Führende Rolle Österreichs in der Quantenforschung 


\author{
Hohe Investitionen in \\ FEE und hohes \\ strategisches Interesse \\ von Nationen und \\ globalen Unternehmen
}

Antizipation zukünftiger

Risiken:

Quantencyberkriege?

\section{Vorschlag weiteres Vorgehen}

Die Dynamik im Feld der Quantentechnologien kann anhand folgender internationaler Trends zusammengefasst werden: hohe Investitionen in Forschung und Entwicklung, unterschiedliche wissenschaftliche Paradigmen in den Forschungsgemeinschaften, sowie unterschiedliche Stadien der Realisierung je nach Anwendungsgebiet. Hinzu kommt das strategische Interesse von Nationalstaaten und globalen Unternehmen, die durch Exportregulierungen, Großinfrastruktur oder exklusive Eigentumsrechte an Technologien und Know-How (IPRs) den Zugriff und die Nutzungsmöglichkeiten in der Zukunft beeinflussen können. Um eine breitere, gesellschaftliche Perspektive zu gewinnen, bietet die Auseinandersetzung mit langfristigen ethischen, rechtlichen und sozialen Aspekten (ELSA) der Quantentechnologie eine Ausgangsbasis. Sehr begrenzt beginnt eine breitere Analyse von Quanten-Innovationen. Das Quantum Flagship Projekt CiViQ setzt z.B. Impulse, indem neben den technologiespezifischen Inhalten auch langfristige ethisch-soziale Aspekte berücksichtigt werden, die mit der QKD-Technologie einhergehen könnten.

Bedrohungsszenarien, wie das internationale Wettrüsten mithilfe militärischer Anwendungen der Quantenkryptographie oder Möglichkeiten von Quantencyberkriegen sind bisher kaum analysiert worden. Diese Risiken zu antizipieren, würde eine Voraussetzung sein, um bei schnellem Fortschritt im Bereich der Quantentechnologien adäquate Governance-Mechanismen frühzeitig zu implementieren. Hierzu könnten in einem Foresight-Prozess alternative Entwicklungen und Änderungssignale („weak signals“) erkundet werden. Das Aufspüren neuer Paradigmen und Ansätze, wie z.B. Quantenbiologie als Schnittstelle von Quantenphysik und Biologie (siehe Quantenbiologie) wurde bereits vorausschauend erprobt ${ }^{12}$; neue Konstellationen mit Zukunftspotenzial könnten ebenso im Rahmen eines Foresight-Prozesses exploriert werden.

\section{Zitierte Literatur}

Arute, F. et al., 2019, Quantum supremacy using a programmable superconducting processor, Nature 574(7779), 505-510.

Elser, D., Marquardt, C. und Leuchs, G., o.J., Quanten-Engineering mit optischer Technologie: Max-Planck-Institut für die Physik des Lichts, Erlangen.

World Economic Forum, 2019, The Global Risks Report 2019: World Economic Forum, .

12 ait.ac.at/ueber-das-ait/center/center-for-innovation-systems-policy/farquest/. 


\section{Biomimikry und Bionik: Designprinzipien aus der Natur}

Biomimikry und Bionik bezeichnen technologische Lösungen, die auf Design-Ansätzen und Prozessen der Natur beruhen. Dieses interdisziplinäre Forschungsfeld wertet Funktionsprinzipien aus der Natur für die Entwicklung von Technologien aus. Die Analyse, wie die Natur im Zuge der Evolution etwa Prozesse und Strukturen in Pflanzen und Organismen hervorgebracht hat, um Energie und Wasser zu gewinnen und zu speichern, wird zur Grundlage für neue Prozessinnovationen. Zugrundeliegende Designprinzipien werden analysiert und auf mögliche Lösungen für zukünftige Technologien untersucht, die strukturell ähnliche Herausforderungen aufweisen: z.B. Temperatur-Isolation, Energiegewinnung, Wassergewinnung, -speicherung oder auch -reinigung. Zusätzlich zu technisch innovativen Lösungen geht es um neue Herangehensweisen und um Prozessinnovationen, die Nachhaltigkeit bereits in das Design integrieren.

Bionik ist bereits in der Architektur etabliert und gewinnt aus den Strukturbildungsgesetzen der Natur Modellierungsansätze und Designmöglichkeiten für neuartige Strukturen (Yuan et al. 2017). Biomimikry ist auf innovative Organisationsstrukturen oder systemische Lösungen ausgerichtet. Neue Aktualität erhalten Bionik und Biomimikry durch die Fortschritte in Simulation und 3D-Druck, die es ermöglichen, komplexe Strukturen und Systeme aus der Natur als Konstruktionsprinzip und Vorlage zu nutzen. Aktuelle Beispiele für die Fertigung der Zukunft sind die etwa von Quallen inspirierte Fortbewegung, das von Tieren inspirierte Roboterdesign - von Roboter-Insekten (Bau/Carde 2015) bis zu Roboterfischen (Neveln et al. 2013) -, die Erforschung des Verhaltens von Tiersystemen (z.B. Ameisen), die helfen könnten, das Internet weiter zu entwickeln oder sogar zu verstehen, wie sich Krebszellen ausbreiten. Ein weiteres Bespiel ist das optische System von Schmetterlingen, das in der Medizintechnik genutzt werden könnte, um während der Operation Krebsgewebe besser erkennen zu können. ${ }^{1}$

Bionik und Biomimikry werden oft primär aus technologischer Sicht betrachtet, allerdings gibt es auch zunehmend Transferansätze, um die Ansätze breiter nutzbar zu machen (vgl. Rovalo et al. 2019). Mit der breiten Diskussion über neue Fertigungstechniken im Kontext der Bioökonomie ergibt sich die Möglichkeit, Bionik/Biomimikry aus der Perspektive branchen- und bereichsübergreifender Problemlösungsansätze zu analysieren und die Designprinzipien der Natur als innovationspolitischen Ansatz zu entwickeln. ${ }^{2}$

\footnotetext{
1 wissenschaft.de/gesundheit-medizin/schmetterlingsaugen-fuer-diekrebschirurgie/.

2 Dies bietet sich insbesondere an, da in Österreich bereit Akteure und Netzwerke zu dem Thema vorhanden sind, sieht z.B: bionikum.at/verein/.
} 


\section{Zitierte Quellen}

Bau, J. und Carde, R. T., 2015, Modeling Optimal Strategies for Finding a Resource-Linked, Windborne Odor Plume: Theories, Robotics, and Biomimetic Lessons from Flying Insects, Integrative and Comparative Biology 55(3), 461-477 < <Go to ISI>://WOS:000360006000011 >; auch veröffentlicht in: Integr. Comp. Biol.

Neveln, I. D., Bai, Y., Snyder, J. B., Solberg, J. R., Curet, O. M., Lynch, K. M. und Maclver, M. A., 2013, Biomimetic and bio-inspired robotics in electric fish research, Journal of Experimental Biology 216(13), 2501$2514<<$ Go to ISI ://WOS:000320305500016 >; auch veröffentlicht in: J. Exp. Biol.

Rovalo, E., McCardle, J., Smith, E. und Hooker, G., 2019, Growing the practice of biomimicry: opportunities for mission-based organisations based on a global survey of practitioners, Technology Analysis \& Strategic Management, 1-17 < doi.org/10.1080/09537325.2019.1634254 >

Yuan, Y. P., Yu, X. P., Yang, X. J., Xiao, Y. M., Xiang, B. und Wang, Y., 2017, Bionic building energy efficiency and bionic green architecture: $A$ review, Renewable \& Sustainable Energy Reviews 74, 771-787 < <Go to ISI>://WOS:000401492900055 >; auch veröffentlicht in: Renew. Sust. Energ. Rev. 


\section{Sprunginnovationen: Neue Konzepte innovationsorientierter Industriepolitik}

Auf europäischer wie auf nationaler Ebene zeichnet sich gegenwärtig eine Renaissance industriepolitischer Konzepte ab. ${ }^{1}$ Zwei globale Dynamiken stehen damit in Zusammenhang: einerseits der Erfolg proaktiver (zum Teil protektionistischer) industriepolitischer Strategien von Ländern, wie z. B. China und den USA; andererseits das rasante Tempo, mit dem digitale Technologien weiterentwickelt werden (siehe bspw. Themen „Kollaborative Plattformen“ oder „KI-Risiken“) und das zu disruptiven Effekten sowie neuen Formen der Marktbeherrschung führen kann. Ein Modell, das diese neuen Dynamiken adressieren soll, ist die Förderung von Sprunginnovationen. Der Sprung betont die Art der erwünschten Innovation: radikale technologische Neuheiten, die eine vollständige Veränderung bestehender Produkte, Dienstleistungen und auch Marktstrukturen nach sich ziehen können.

In Deutschland soll zukünftig eine Agentur für Sprunginnovationen ${ }^{2}$ Anreize für die Durchführung „neuer, richtungsweisender, wagemutiger Forschungs- und Entwicklungsprojekte" setzen - wie es im Bericht zu Sprunginnovationen der Deutschen Akademie der Technikwissenschaften heißt (Harhoff et al. 2018). Mit einem personenzentrierten Ansatz, bei dem "hochkompetente und kreative Innovationsmanagerinnen und -manager" eingesetzt werden sollen, die zeitlich befristet tätig sind und besondere Handlungsfreiräume genießen, soll eine Beschleunigung der Umsetzung von Forschung in die Praxis institutionell ermöglicht werden. ${ }^{3}$ Der Diskurs um neue Wege zur Förderung bahnbrechender Innovationen ist nicht auf den deutschsprachigen Raum beschränkt; gemeinsam mit Frankreich setzt sich Deutschland dafür ein, dass mit dem neuen EU-Forschungsrahmenprogramm dazu beigetragen wird, dass auf allen Ebenen (Regionen, Mitgliedstaaten, EU insgesamt) aufeinander abgestimmte Initiativen für Forschung und Innovation gestartet werden können (Deutscher Bundestag 2018). In diesem Zusammenhang steht auch die Forderung der Deutschen Bundesregierung an den Europäischen Innovationsrat (EIC), die Innovations- und Wachstumsfinanzierung von technologieintensiven Start-ups zu ermöglichen, da diese an mitgliedstaatengetriebene Agenturen zur Förderung von Sprunginnovationen anschlussfähig wäre (Deutscher Bundestag 2018).

\footnotetext{
1 Vgl. hierzu die Debatte über das sogenannte „Altmaier-Papier“ (2019) in Deutschland oder die Neue Industriepolitische Strategie der EU (2017), die in Folge zur Lancierung der sogenannten IPCEIs (Important Projects of Common European Interest) geführt hat.

2 sprind.org.

3 bmbf.de/de/bundeskabinett-beschliesst-agentur-zur-foerderung-vonsprunginnovationen-6817.html.
} 
In Österreich wird die Diskussion um die Förderung radikaler und bahnbrechender Innovation schon seit längerem geführt. An prominenter Stelle fordert der FWF Präsident mehr Geld für eine risikofreudigere Wissenschafts- und Forschungskultur für Österreich ${ }^{4}$, aber auch der Rat für Technologie und Forschung hat bereits 2016 eine Studie veröffentlicht, die Ansatzpunkte zur Stärkung des innovativen Potenzials in österreichischer Forschung und Entwicklung, v. a. im Bereich radikaler Innovationen und risikobehafteter Forschung, analysiert (Warta/Dudenbostel 2016). Eine weitere, 2020 veröffentlichte, Studie des Rats verweist erneut auf die hohe Relevanz radikaler Innovatoren für Österreich und benennt konkrete innovationspolitische Maßnahmen (Dachs, Kalcik, \& Wasserbacher, 2019). Auch die Ausrichtung der "COMET - Competence Centers for Excellent Technologies" Förderungsprogrammlinie baut auf wesentliche Elemente risikoaffiner Innovationsförderung: Ziel ist die Erforschung zukunftsweisender Themen, das Setzen neuer Forschungsimpulse, ein verstärkter Technologietransfer und die Erhöhung der Innovationsfähigkeit von Unternehmen. Explizit verweist die Innovationsstrategie der WKÖ auf Sprunginnovationen. ${ }^{5} \mathrm{Ob}$ und ggf. wie Sprunginnovationen zukünftig in Österreich weiter aufgegriffen und wie radikal neuartige Lösungen durch die öffentliche Forschungsförderung vorangetrieben werden könnten, ist derzeit noch eine offene Frage.

\section{Zitierte Literatur}

Dachs, B., Kalcik, R., \& Wasserbacher, D. (2019). Radikale Innovation und Firmenalter. Im Auftrag des Rates für Forschung und Technologieentwicklung. Wien. AIT-ISP-Report 21. rat-fte.at/files/rat-ftepdf/publikationen/2020/200109_Bericht\%20radikale\%20Innovation\%20 und\%20Firmenalter_AIT.pdf.

Deutscher Bundestag, 2018, Geplante Gründung der Agentur zur Förderung von Sprunginnovationen, Drucksache 19/5679: Antwort der Bundesregierung.

Harhoff, D., Kagermann, H. und Stratmann, M., 2018, Impulse für Sprunginnovationen in Deutschland; in Reihe: acatech Diskussion, München: Herbert Utz Verlag.

Warta, K. und Dudenbostel, T., 2016, Radikale Innovationen - Mehr Freiraum für innovative und risikobehaftete Forschung. Endbericht, Frankfurt am Main: technopolis group.

4 derstandard.at/2000045445359/FWF-Praesident-Wissenschaft-muss-auchunbequem-sein.

5 Vgl. wko.at/site/innovate-austria/innovationsstrategie/wkoinnovationsstrategie.pdf. 


\section{Geldlose Tauschsysteme: Zeitbanken}

Zeitbanken sind sozio-technische Systeme, die Dienstleistungen mit Zeit anstatt mit Geld vergüten (Carroll et al. 2016). Sie zielen generell darauf ab, künftigen Herausforderungen wie dem demografischen Wandel und daraus resultierenden Problemlagen entgegen zu wirken. Vor allem im Bereich der Altersvorsorge (z. B. Betreuungsbedürfnisse der älteren Generation) spielt die Idee des Zeittausches eine Rolle. Eine Zeitbank übersetzt Zeit in Zeitgutschrift für persönliches Engagement und praktische Hilfe, die später für eigene Bedürfnisse genutzt werden kann. Dieses System zum geldlosen Austausch von Dienstleistungen verspricht, Anreize für zeitlich limitierte oder regelmäßige Engagements zugunsten der Allgemeinheit zu schaffen. Darüber hinaus sollen Zeitbanken die Entstehung sozialer Netzwerke fördern und die Gesundheits-, sowie Pflege- und Betreuungskosten senken.

In Österreich gibt es bereits über 40 regionale Zeitbanken und Tauschkreise (Höllhumer \& Trukeschitz, 2016), sowie überregionale Time Bank Angebote $^{1}$. In der Schweiz ${ }^{2}$ wird die Schaffung eines regulativen Rahmens für die Organisation und Administration von Zeitbanken, sowie die Erstellung von Leistungskatalogen im Parlament diskutiert. Dabei ist die Frage zentral, ob das Modell des Zeittauschsystems überhaupt öffentlich/ staatlich organisiert sein soll, oder basierend auf dem Modell der Nachbarschaftshilfe im lokalen Rahmen verankert sein soll. Die Auswirkungen eines solchen Systems auf bereits bestehendes soziales Engagement in Vereinen oder Gemeinden bleibt fraglich.

Heute nutzen die Akteure von Zeitbanken zum Tracking von Zeitbank-Austauschvorgängen spezielle Software und auch Smartphone-Apps (Han et al. 2015). Diese ermöglicht die Dokumentation der Aktivitäten von TeilnehmerInnen: was können sie anbieten, was möchten sie im Gegenzug erhalten und was wurde ausgetauscht (z. B. wie viele Zeiteinheiten bzw. Personenstunden). Neben gängiger Software für das Management von Zeitbanken (z. B. TimeRepublik, hOurWorld Time and Talents, Community Forge, Community Weaver 3) wäre es vorstellbar, dass zukünftig auch die Blockchain-Technologie für Time Banking eingesetzt wird.

\section{Zitierte Quellen}

Carroll, J. M., Shih, P. C., Han, K. und Kropczynski, J., 2016, Coordinating community cooperation: Integrating timebanks and nonprofit volunteering by design, International Journal of Design 11(1), 51-63.

Han, K., Shih, P. C., Bellotti, V. und Carroll, J. M., 2015, It's Time There Was an App for That Too: A Usability Study of Mobile Timebanking, International Journal of Mobile Human Computer Interaction 7(2), 1-22; auch veröffentlicht in: Int. J. Mob. Hum. Comput. Interact.

\footnotetext{
1 erste-time-bank.org.

2 parlament.ch/de/ratsbetrieb/suche-curia-vista/geschaeft?Affairld=20150484.
} 


\section{FinTechs - Revolution des Bankenwesens?}

\section{Zusammenfassung}

„Bankgeschäfte sind notwendig, Banken nicht" - hat Bill Gates schon 1994 vorausgesagt. Es war zu einer Zeit, in welcher die sogenannten FinTechs noch nicht existierten und der weltweite Finanzsektor allein von Banken und Versicherungen dominiert wurde. Jahre später stehen diese Unternehmen tatsächlich vor der Frage: Sind sie überhaupt noch notwendig? Und wenn ja, in welcher Form? Digitale Transformation hat auch die konservative Finanzbranche erreicht - immer mehr Kundlnnen gehen nicht mehr in die Bankfiliale oder lassen Roboter statt FinanzexpertInnen entscheiden, welche Aktien sie kaufen sollen. Unter dem Begriff „FinTech“ versteht man eine Vielzahl neuer Ideen, wie durch den Einsatz von digitalen Technologien Finanzdienstleistungen angeboten und verbessert werden können. Dabei wird ein breites Spektrum abgedeckt: So spielen neben der Anwendung von innovativen Technologien, wie z.B. Blockchain oder Künstliche Intelligenz, auch die Verbesserung von kundennahen Prozessen und neue Geschäftsmodelle eine große Rolle. Diese Innovationen werden oft von FinTech-Start-ups vorangetrieben, die kompetitiv oder auch komplementär zu den klassischen Banken agieren.

\section{Überblick zum Thema}

Üblicherweise werden Bankgeschäfte in verschiedene Kategorien eingeteilt, z.B. Vermögensmanagement, Zahlungsverkehr, Finanzierung, Versicherung, u.v.m.; so werden auch FinTech-Lösungen in diesen Kategorien angeboten.

Im Zuge der Finanzkrise 2008 konnten sich zahlreiche FinTechs im Bereich Vermögensmanagement etablieren. Ausgehend vom angloamerikanischen Raum erreichte dieses sogenannte "Asset Management 2.0" schließlich auch europäische Länder, wo eine Vielzahl neuer Anbieter gegründet wurden. Dazu zählt z.B. Wikifolio', eine im Jahr 2008 gegründete, österreichische Social Trading Plattform. Social Trading ist eine Form der Anlageberatung und Vermögensverwaltung, bei der Informationen und Expertisen hinsichtlich bestimmter Anlageentscheidungen zwischen Anlegerlnnen ausgetauscht werden. Dabei veröffentlichen Anlegerlnnen ihre Meinungen zu einzelnen Wertpapieren der gesamten Portfolien auf speziellen Plattformen, wie eben Wikifolio, was wiederum andere AnlegerInnen einsehen, kommentieren oder mit dem eigenen Vermögen nachbilden können. Auf dieser Weise werden die Anlageentscheidungen zeitnah offengelegt und mit Einschätzungen versehen, was eine bestimmte Transparenz des Anlageprozesses verspricht. Eine weitere FinTech-Innovation im Bereich Vermögensmanagement ist die automatisierte Anlageempfehlung, oder „Robo-Advice“. „Robo“ bezeichnet dabei einen automatisierten

FinTechs im Bereich

Vermögensmanagement

1 wikifolio.com. 
FinTechs im Bereich Zahlungsverkehr
Prozess ohne menschlichen Einfluss, wobei vor allem mathematische Algorithmen zur Findung von Investitionsentscheidungen genutzt werden. „Advice“ bezieht sich auf den Wealth Management Service an sich, der automatisch über Online- oder mobile Kanäle angeboten bzw. genutzt wird. Die Robo-Advisors können sich stark durch den zugrunde liegenden Ansatz unterscheiden. Z.B. geben die Robo-Advisors der ersten Generation nur Vorschläge für Portfolioallokationen, wobei KundInnen die vorgeschlagenen Anlagestrategien selbst umsetzen müssen. Die jüngste Generation der Robo-Advisor basiert auf $\mathrm{KI}$ und tätigt selbst Investitionen im Namen der Kundlnnen. Der Robo-Advisor-Markt wächst weltweit, ${ }^{2}$ aber wie sicher und vertrauenswürdig sind eigentlich Robo-Advisors? Es handelt sich um relativ neue Player am Markt, was einen Vertrauensvorschuss der KundInnen voraussetzt, da sie bei der Veranlagung auf oft nicht transparente automatische Prozesse and Algorithmen angewiesen sind, für die es noch keine langfristigen Erfahrungswerte gibt. Um die Vertrauensbasis zu stärken, setzten viele AnbieterInnen auf eine Robo-Hybrid-Beratung, bei der die Kundlnnen zusätzlich durch menschliche Beratung unterstützt werden. In Hinblick auf Sicherheitsaspekte unterliegen die traditionellen Beraterlnnen und die Robo-Advisors im Wesentlichen ähnlicher Regulierung, die darauf abzielt, die KundInnen vor falschen Entscheidungen zu schützen.

Vielfältige FinTech-Lösungen gibt es auch in dem Bereich Zahlungsverkehr. Viele FinTechs, übereinstimmend mit der EU-Direktive PSD $\|^{3}-$ die die Entwicklung innovativer Online- und mobiler Zahlungsmöglichkeiten reguliert - entwickeln hauptsächlich neue Online-Bezahldienste sowie verbesserte Schnittstellen für Benutzerlnnen. In den letzten Jahren verändert sich der Modus des Bezahlens durch Kryptowährungen und Blockchain-Technologie (siehe Thema „Vertrauenswürdige Blockchains") deutlich. Blockchain ist damit einerseits eine disruptive Entwicklung für das etablierte Finanzwesen, da sie keine Intermediäre benötigt bzw. sie ersetzt. Andererseits bietet diese Technologie auch für Banken viele Vorteile, z.B. kann Wertpapierhandel mit Einsatz der Blockchain peer-to-peer (P2P) erfolgen und ist nicht mehr auf die zahlreichen Zwischenstationen von Haus- bis Depotbanken angewiesen. Um die Technologie für sich zu nutzen, nehmen Banken bei der aktuellen Weiterentwicklung von Blockchains oft eine Vorreiterrolle ein. Die FinTech-Entwicklungen im Bereich Zahlungsverkehr und Payment haben auch das Potential, die finanzielle Inklusion von Menschen in Entwicklungsländern voranzutreiben. Laut Global Findex Database, hatten 2017 rund 1,7 Milliarden Menschen keinen Zugang zu klassischen Finanzdienstleistungen (Demirguc-Kunt et al. 2018). Durch hohe Kosten, einen Mangel an Servicestellen und Bankfilialen, sowie Herausforderungen beim Identitätsnachweis, bleiben gerade Menschen in Entwicklungsländern oft ohne Zugang zu Finanzdienstleistungen, d.h. „unbanked“. Um die „unbanked“ Bevölkerung zu adressieren, stellen einige FinTechs sogenannte „Mobile-First“-Bezahlsysteme zur Verfügung, wel-

\footnotetext{
2 kommalpha.com/download. php?id=41.

3 eur-lex.europa.eu/legal-content/DE/ALL/?uri=CELEX\%3A32015L2366.
} 
che in Zusammenarbeit mit Telekomanbietern Menschen ohne Bankkonto bargeldloses Zahlen über das Mobilabonnement ermöglichen. Ein Beispiel dafür ist das Bezahlsystem „M-Pesa“, das von knapp zwei Drittel der kenianischen Bevölkerung genutzt wird, da Mobiltelefone in Kenia, wie in vielen afrikanischen Ländern, viel weiter verbreitet sind als Bankkonten.

Auch der Versicherungsbereich wird von digitalen Technologien maßgeblich beeinflusst. Der Begriff „InsurTech“ fasst eine Reihe an technologiebasierten Lösungen, die entlang der gesamten Versicherungswertschöpfungskette - von der Produktentwicklung bis hin zur Schadenabwicklung - eingesetzt werden können. Nicht nur neue Technologien, sondern auch innovative Geschäftsmodelle spielen dabei eine große Rolle; z.B. werden beim P2P-Versicherungsmodell Versicherte online zu Gruppen zusammengeschlossen, deren Mitglieder sich gegenseitig finanziell unterstützen. Von den gezahlten Versicherungsbeiträgen fließt ein Teil in einen Rückzahlungstopf. Dieses Geschäftsmodell hat sich mittlerweile weltweit etabliert, wobei auch hier die Herausforderungen von P2P-Märkten sichtbar werden. So kann es für junge, noch nicht etablierte AnbieterInnen problematisch sein, eine ausreichende Mitgliederzahl mit einem ähnlichen Risikoprofil anzuwerben. ${ }^{4}$ Unterschiedliche regulatorische Rahmenbedingungen im stark vom jeweiligen Länderkontext abhängigen Versicherungswesen sind für die P2P-InsurTechs, v.a. für den breiten Roll-out ihrer Geschäftsmodelle herausfordernd. ${ }^{5}$

\section{Relevanz des Themas für das Parlament und für Österreich}

FinTechs nehmen an Bedeutung zu. Laut eines KPMG-Berichts erreichten die Investitionen in FinTech-Unternehmen im Jahr 2018 weltweit 111,8 Milliarden Dollar (Pollari und Ruddenklau 2019). Eine weitere Umfrage zeigt, dass das Thema FinTech von den klassischen Finanzinstituten ernst genommen wird, wobei FinTech-Innovationen bereits jetzt schon in die Angebote von etablierten Marktakteuren einfließen (PwC 2019). Neben den etablierten FinTech-Standorten entsteht in den letzten Jahren auch in Österreich eine bedeutsame FinTech-Szene. Die digitale Bank „Number 26“6, die 2013 in Österreich als FinTech gegründet worden ist, verfügt jetzt bereits über fünf Millionen KundInnen in 25 Ländern weltweit. Um weitere österreichische FinTechs zu unterstützen, hat das Bundesministerium für Finanzen den FinTech-Beirat eingerichtet. Ziel ist es, attraktive Rahmenbedingungen (siehe Thema „Reallabore“) für FinTechWeiterentwicklungen in Österreich zu schaffen. Eine weitere österreichische Einrichtung ist die FinTech-Kontaktstelle bei der FMA - die Behörde steht den FinTech-Start-ups bei konkreten aufsichtsrechtlichen Fragen in erster Linie beratend zur Seite. ${ }^{7}$ Nicht nur Start-ups, sondern auch viele

\footnotetext{
4 home.kpmg/xx/en/home/insights/2017/09/is-p2p-insurance-a-sustainablebusiness-model.html.

5 moneyunder30.com/p2p-insurance.

6 n26.com.

7 fma.gv.at/querschnittsthemen/fintechnavigator/.
}

Versicherungsbereich InsurTech 
etablierten Banken in Österreich beschäftigen sich intensiv mit dem Thema FinTech und kooperieren dabei immer mehr mit jungen Unternehmen. Im Zusammenhang mit digitalen Technologien, die bei den FinTechs zum Einsatz kommen, bestehen Chancen aber auch Risiken. Z.B. kann Big Data Analytics (BDA) beim Gestalten von kundenorientierten Dienstleistungen, sowie auch bei der Prozessoptimierung Vorteile bringen. Der immer umfassendere Einsatz von BDA in der Finanzbranche erfordert die Berücksichtigung vielfältiger rechtlicher, sozialer und ethischer Aspekte. Aktuelle Debatten rund um das Thema fokussieren auf Datenschutz, Datensouveränität sowie auf Vermeidung von Diskriminierung bei automatischen Entscheidungen (Fang und Zhang 2016). Datenschutz ist auch bei innovativen Technologien der InsurTechs, wie z.B. dem Internet der Dinge und den dabei anhand von Sensoren gesammelten Daten, ein zentrales Thema. Einerseits ermöglicht die umfassende Nutzung von Daten eine flexiblere Preisgestaltung und die individuelle Anpassung unterschiedlicher Versicherungsprodukte. Andererseits ergeben sich daraus besondere Herausforderungen in Hinblick auf Datenschutz und die gesellschaftlich vertretbare Nutzung personenbezogener Daten.

\section{Vorschlag weiteres Vorgehen}

Wesentlich wäre es, die Herausforderungen digitaler Technologien in der Finanzbranche - insbesondere bezüglich Datensouveränität und der Transparenz bei der Datennutzung - näher zu beleuchten und in Hinblick auf ihre sozialen, ethischen und rechtlichen Dimensionen zu hinterfragen. Ob zukünftig bestimmte Bereiche des Finanzsektors von FinTechs dominiert werden oder ein kooperatives Szenario mit unterschiedlichen Akteuren für das Finanzwesen wahrscheinlich ist, bleibt vorerst offen. Eine detailliertere Zukunftsanalyse, die anhand explorativer Szenarien Trends und mögliche Weiterentwicklungen für FinTech aufzeigt, könnte zukünftige Anwendungspotenziale (z.B. FinTech für die Kreditvergabe), sowie Barrieren (z.B. in Hinblick auf eine europäische Harmonisierung von Standards und regulatorischen Experimentierräumen) adressieren.

\section{Zitierte Literatur}

Demirguc-Kunt, A., Klapper, L., Singer, D., Ansar, S. und Hess, J. (2018). The Global Findex Database 2017: Measuring financial inclusion and the fintech revolution. The World Bank.

Fang, B. und Zhang, P. (2016). Big data in finance. In Big data concepts, theories, and applications (pp. 391-412). Springer, Cham.

Pollari, I. und Ruddenklau, A. (2019). The Pulse of Fintech 2018: Biannual Global Analysis of Investment in Fintech. KPMG International Cooperative.

PwC (PricewaterhouseCoopers) (2019) Global FinTech Report, pwc.com/gx/en/industries/financial-services/assets/pwc-global-fintechreport-2019.pdf. 


\section{Europäische Resilienz in Krisenzeiten}

\section{Zusammenfassung}

Die aktuelle, weltweite Krise, die durch die Covid-19-Pandemie ausgelöst wurde, macht wie kaum ein Ereignis bislang die große Verwundbarkeit der europäischen Staaten und damit auch Österreichs deutlich. Die internationale Vernetzung und Arbeitsteilung im Bereich der Gütererzeugung und der Dienstleistungserbringung, die sich über die vergangenen Jahrzehnte entwickelt hat, führt zu mannigfachen und teils sehr fragilen $A b-$ hängigkeiten zwischen den verschiedenen Staaten und Regionen. Bei einzelnen Warenströmen wurde das schon in der Vergangenheit deutlich (z.B. Erdölkrise 1973). Die durch die Pandemie ausgelösten weltweiten Grenzschließungen und Marktabschottungen machen die Vulnerabilität einzelner Staaten heute umso deutlicher: So werden viele Waren (z.B. Arzneien und Medizinprodukte, sogar viele, prinzipiell überall verfügbare Grundstoffe) gar nicht mehr in Europa produziert, manche Arbeitsleistungen (z.B. im Pflegebereich, im Transportwesen, in der Landwirtschaft) nur von Grenzgängern oder internationalen Pendlerlnnen erbracht und viele Kommunikationsdienstleistungen (z.B. Video-Konferenz-Plattformen) nur aus dem Ausland, oftmals von US-amerikanischen Firmen angeboten. Vor diesem Hintergrund stellt sich die strategische Frage, ob, und wenn $\mathrm{ja}$, in welcher Weise und in welchen besonders kritischen Bereichen sich Österreich bzw. Europa für die Zukunft souveräner, also unabhängiger von den internationalen Güter-, Dienstleistungs- und Arbeitsmärkten machen könnte, um in Krisen wie der aktuellen resilienter zu sein.

\section{Überblick zum Thema}

Es ist Mainstream-Auffassung, dass Globalisierung der Weltwirtschaft für die meisten Länder zu enormen Wohlstandsgewinnen geführt hat. Organisatorische, rechtliche, ökonomische und technologische Innovationen konnten bisherige Herausforderungen im Kleinen und im Großen meistern und haben zur Optimierung des Weltwirtschaftssystems beigetragen. Weltweite Krisen wie die Erdölkrise der 1970er-Jahre oder zuletzt die Weltwirtschafts- und Eurokrise ab 2007 konnten gemeistert werden. Auch Pandemien, die in den letzten 20 Jahren aufgetreten sind, waren in ihren Auswirkungen nicht so dramatisch. Die Covid-19-Krise stellt die Staatengemeinschaft jedoch vor eine völlig neue Herausforderung, weil sie nicht nur einen Sektor (z.B. Energie oder Banken) oder einzelne Regionen betrifft, sondern viel umfassender ist. Schon nach wenigen Tagen und Wochen stellte sich mit großer Klarheit heraus, dass die in normalen Zeiten als positiv wahrgenommenen internationalen Verflechtungen unter Pandemiebedingungen höchst problematisch sind. Nicht nur hat die internationale Mobilität die Pandemie in kürzester Zeit in jeden Winkel der Erde gebracht, die in der Folge ergriffenen Abschottungsmaßnahmen auf vielen Ebenen haben zum Zusammenbruch von Lieferketten aller Art (nicht 
Produktion von

Arzneimitteln und

Medizinprodukten

Grenzüberschreitender

Verkehr von

Arbeitskräften in

kritischen Bereichen

Grenzüberschreitender [Güter]verkehr nur Waren, auch Arbeitskraft) geführt. Das Schengen-Abkommen, als ein zentraler Baustein der EU, wurde innerhalb kürzester Zeit durch die Maßnahmen zur Bekämpfung des Covid-19 ausgesetzt. Auch wenn das gesamte Ausmaß der Folgen dieser weltumspannenden Krise heute (Mai 2020) noch keineswegs absehbar ist, können doch schon einige kritische Bereiche benannt werden, in denen die übliche Verfasstheit der europäischen Staatengemeinschaft zu gravierenden Problemen führt. Diese sollen in der Folge überblicksartig dargestellt werden.

Viele Pharmazeutika und Medizinprodukte werden unter Ausnutzung von Kostendifferentialen nicht mehr in Österreich bzw. in Europa produziert, obwohl das entsprechende Knowhow in der Regel auch hierzulande vorhanden ist. Das führt, wie in der Covid-19-Krise eindrücklich erfahren werden konnte, dazu, dass der Nachschub mit vielen, teils lebenswichtigen Medikamenten für Europa zeitweise unterbrochen war und ist. Selbst Atemschutzmasken (einfache wie hochwertige für das medizinische Personal) werden praktisch nicht mehr hierzulande produziert. Knappheit herrscht auch hinsichtlich der notwendigen Rohstoffe, um Covid-19-Tests durchführen zu können. Die Situation wird auch dadurch verschärft, dass einzelne Staaten Exportverbote auf teils bereits erworbene Medizinprodukte verhängen. Besorgniserregend ist zudem, dass Staaten und teilweise sogar einzelne Bundesstaaten eines Landes untereinander in ein Bieterverfahren treten (müssen), um an die notwendeigen Medizinprodukte zu gelangen. ${ }^{1}$ Für politisches Aufsehen hat auch der letztlich gescheiterte Übernahmeversuch eines deutschen Pharmaunternehmens durch die Vereinigten Staaten gesorgt. Das Unternehmen arbeitet gerade an der Entwicklung eines Impfstoffes gegen COVID-19 und die USA wollte sich das Exklusivrecht auf den potentiellen Impfstoff sichern. ${ }^{2}$ Die Beispiele verdeutlichen, welche weitreichenden und nicht vorhersehbaren Konsequenzen solche Krisensituationen hervorrufen können.

Insbesondere in kritischen Sektoren wie der Pflege, in der Landwirtschaft (Erntehilfe) und im Gütertransportwesen hat die aktuelle Krise gezeigt, dass ein funktionierender Arbeitsmarkt sehr voraussetzungsvoll ist. Auch nur vorübergehend geschlossene Grenzen haben deutlich gemacht, dass etwa die Pflege in Österreich zusammenbricht, die Gemüseernte zum Erliegen kommt und zu wenige Fahrerlnnen für Lastkraftwagen im Inland zur Verfügung stehen.

Neben den Mobilitätseinschränkungen für Arbeitskräfte führt der Lockdown in den einzelnen Ländern zeitweise auch zu einem eingeschränkten Güterverkehr. Es kam zu kilometerlangen LKW-Staus an den Grenzen. Auch der stillgelegte Personenflugverkehr hat Auswirkungen auf die Güterversorgung in verschiedenen Branchen und Sektoren. Zusätzlich be-

1 Der Gouverneur von New York beschreibt ein Bieterverfahren um Beatmungsgeräte wie in einem Online-Auktionshaus, siehe theguardian.com/usnews/2020/mar/31/new-york-andrew-cuomo-coronavirus-ventilators.

2 theguardian.com/us-news/2020/mar/15/trump-offers-large-sums-for-exclusiveaccess-to-coronavirus-vaccine. 
wirkt die weitgehende Stilllegung des Flugverkehrs auch eine Verschlechterung der Qualität der Wetterprognosen, da Flugzeuge auch eine wichtige Funktion bei der Erhebung der Wetterdaten haben. Hier wird deutlich, dass der Personenflugverkehr auch für andere Bereiche kritisch ist, die nun kompensiert werden müssen, etwa über vermehrten Einsatz von Wetterballons ${ }^{3}$ oder die Zunahme der reinen Frachtflüge ${ }^{4}$.

Das im Zuge der Covid-19-Krise von vielen Staaten verordnete Homeoffice hat die Kommunikationsinfrastruktur, die in Europa in Normalzeiten als im weltweiten Vergleich besonders gut ausgebaut gelten kann, an ihre Leistungsgrenzen gebracht. Darüber hinaus sind technologische (und ökonomische) Abhängigkeiten von bestimmten, in der Regel US-amerikanischen Plattformen zu Tage getreten und stärker geworden (siehe dazu bereits das Thema „Digitale Souveränität" sowie Nentwich et al. 2019). Da viele private Personen zu Hause nicht dieselben IT-Sicherheitsmaßnahmen erfüllen können wie Unternehmen, entstehen möglicherweise Sicherheitslücken, die von Cyberkriminellen ausgenutzt werden können.

\section{Relevanz des Themas für das Parlament und für Österreich}

Die Frage der österreichischen bzw. europäischen Souveränität in kritischen Bereichen hat eine große strategische und gesamtstaatliche Bedeutung. Eine konsistente und erfolgversprechende Strategie zur Erreichung von mehr österreichischer bzw. kontinentaler Unabhängigkeit, Autarkie und Resilienz muss sehr viele Aspekte, Sichtweisen und Bereiche gleichzeitig berücksichtigen. Die Schweiz hat beispielsweise ein eigenes Bundesamt für wirtschaftliche Landesversorgung: Es greift „im Falle eines Versorgungsengpasses [...] mit gezielten Massnahmen in das Marktgeschehen ein, um entstandene Angebotslücken zu schliessen". ${ }^{5}$ Diese Governancestruktur könnte als Modell dienen und an die aktuellen Herausforderungen angepasst werden. Alternative Ansätze könnten darauf abzielen, mehr Flexibilität in bestehende Produktionsprozesse einzubauen, um sich vorab definierten Situationen kurzfristig und rasch entsprechend den Krisenmanagementplänen anpassen zu können. Aktuelle Beispiel dafür sind u.a. die Anfertigung von Mund-Nasen-Schutzmasken durch einige Textilverarbeitungsbetriebe oder die Herstellung von Desinfektionsmitteln durch Destillerien oder Parfumhersteller. Darüber hinaus wird auch eine Re-Regionalisierung zumindest mancher, strategisch bzw. im Krisenfall besonders kritischer Produkte bzw. Produktgruppen als Lösungsweg diskutiert. ${ }^{6}$
Kommunikationsinfrastruktur

3 zamg.ac.at/cms/de/wetter/news/zusaetzliche-aufstiege-von-wetterballons-umausfall-von-flugzeug-daten-auszugleichen.

4 forbes.com/sites/willhorton1/2020/03/31/cargo-aircraft-roar-to-life-reachingrecord-utilization-as-coronavirus-creates-urgent-demand-for-airfreight/\#6e522498bdd7.

5 bwl.admin.ch/bwl/de/home/das-bwl/auftrag.html.

6 derstandard.at/story/2000117234618/schluss-mit-globalisierung-warum-derabgesang-verfehlt-sein-duerfte. 
Es bedarf jedenfalls eines Strategieprozesses, der alle gesellschaftlichen Sektoren einbezieht, um die notwendigen Abwägungen zwischen unterschiedlichen Interessen bestmöglich vorzunehmen. Dabei gilt es auch, die mögliche Kehrseite einer neuen Souveränität im Blick zu halten. Es könnte im schlimmsten Fall zu „nationalem Egoismus“ und dadurch zu einer zunehmenden zwischenstaatlichen Fragmentierung führen, welche ein koordiniertes und geschlossenes Vorgehen bei globalen Herausforderungen erschweren würde. Das Parlament ist der geeignete Ort, um diese Fragen adäquat behandeln zu können und zu moderieren.

\section{Vorschlag weiteres Vorgehen}

Zur Fundierung der oben angesprochenen Debatte im Parlament und in der Gesellschaft erscheint es sinnvoll, in einem ersten Schritt eine umfassende Bestandsaufnahme der mannigfachen Abhängigkeiten in den Güter-, Dienstleistungs- und Arbeitsmärkten zu erstellen. Es handelt sich um eine interdisziplinäre Aufgabe, die nicht nur die primär angesprochene ökonomische Perspektive einbezieht, sondern auch politische, kulturelle, rechtliche und gesellschaftliche Abhängigkeiten in die Betrachtungen einbezieht, wie z.B. internationale Handelsverträge, die Kompetenzverteilung innerhalb Österreichs und innerhalb der EU, spezifische Präferenzen und Lebensgewohnheiten der Österreicherlnnen usw. - was genau einbezogen werden sollte, müsste im Scoping geklärt werden.

\section{Zitierte Literatur}

Nentwich, M., Jäger, W., Embacher-Köhle, G. und Krieger-Lamina, J., 2019, Kann es eine digitale Souveränität Österreichs geben? Herausforderungen für den Staat in Zeiten der Digitalen Transformation. ITA Manu:scripts Nr. ITA-19-01 epub.oeaw.ac.at/ita/ita-manuscript/ita_19_01.pdf 


\section{Bergbau im All}

Asteroiden und Planeten des Sonnensystems könnten in Zukunft als Quelle für Edelmetalle nutzbar werden und über einen Bergbau im Weltall abgebaut werden. Damit könnten nicht nur Rohstoffe für die Erde gewonnen werden, sondern die Nutzung der lokal verfügbaren Ressourcen könnte die Wirtschaftlichkeit und Nachhaltigkeit zukünftiger Weltraummissionen strukturell verbessern: Über 3D-Druck/additive Fertigung würden die Ressourcen für die Herstellung von Ersatzteilen, Werkzeugen und Großstrukturen direkt im All genutzt werden (Lietaert et al. 2018). Obwohl es nicht klar ist, ob ein wirtschaftlich und ökologisch nachhaltiger Bergbau im All überhaupt möglich ist und obwohl sich der Weltraumbergbau zurzeit in einer konzeptionellen Phase befindet (Probst et al. 2016), sind in vielen Ländern bereits Gesetze erlassen, um einen rechtlichen Rahmen für diese Zukunftstechnologie bereitzustellen. Damit beginnt ein Wettlauf um diese Rohstoffe und Bodenschätze im All, da der Wert der Metalle auf einzelnen Asteroiden bereits in Trillionen Euro geschätzt wird. ${ }^{1}$ Führende Weltraumstaaten wie die USA und Russland haben bereits Gesetze verabschiedet und auch in europäischen Ländern wie Finnland und Spanien wurden nationale Weltraumgesetze erlassen. ${ }^{2}$ Luxemburg hat Mitte 2017 einen rechtlichen Rahmen für den möglichen Abbau von Rohstoffen auf Asteroiden im Weltraum entwickelt und im September 2018 eine entsprechende Agentur gegründet, um eine führende Rolle bei der wirtschaftlichen Nutzung des Weltraums einzunehmen. ${ }^{3}$ In Deutschland fordert der Industrieverband BDI ein nationales Weltraumgesetz. ${ }^{4}$

International ist noch unklar, wie die zukünftige Nutzung der Ressourcen im Weltall aussehen wird. Zwar haben die Vereinten Nationen seit 1967 mit fünf Weltraumverträgen einen verbindlichen völkerrechtlichen Rahmen entwickelt. Danach sind alle Staaten frei, den Weltraum zu nutzen, aber es besteht ein Aneignungsverbot. Wie ein regulativer Rahmen zwischen Nutzungsfreiheit und Aneignungsverbot zukünftig aussehen kann, ist bisher offen. Ob die aktuell beschlossenen Gesetze mit dem Völkerrecht im Einklang stehen, ist ebenso unklar wie die Notwendigkeit weiterer Rege-

1 futurezone.at/science/space-mining-10000-asteroiden-im-visier-vom-bergbaustart-up/400004264.

2 issuu.com/bdi-berlin/docs/20180801_position_bdi_rohstoffpolit; zur aktuellen Durchführungsverordnung der USA siehe space.com/trump-moon-miningspace-resources-executive-order.html.

3 Für Informationen zum rechtlichen Rahmen siehe: spaceagency.public.lu/en/agency/legal-framework.html.

4 spiegel.de/wirtschaft/unternehmen/weltraum-bergbau-gesetz-deutscheindustrie-will-rohstoffe-im-all-ausbeuten-a-1214970.html. 
lungen zum Schutz des Weltalls (vgl. Leon 2018). ${ }^{5}$ Wenn sich die UNMitglieder auf ein internationales Regime einigen, könnte die Nutzung nach klaren und nachhaltig wirksamen Regeln organisiert werden. Damit würden auch Staaten ohne direkten Zugang zum Weltall nicht ins Hintertreffen geraten.

Der Aufbau von Wissensbeständen in Österreich zu den wirtschaftlichen, technologischen und rechtlichen Zukunftsoptionen würde es ermöglichen, im Fall einer hohen technologischen und international politischen Dynamik strategisch vorzugehen.

\section{Zitierte Quellen}

Leon, A. M., 2018, Mining For Meaning: An Examination Of The Legality Of Property Rights In Space Resources, Virginia Law Review 104(3), 497-546.

Lietaert, K., et al., 2018, Meteorite as raw material for Direct Metal Printing: A proof of concept study, Acta Astronautica 143, 76-81.

Probst, A., Peytavi, G. G., Eissfeller, B. und Forstner, R., 2016, Mission concept selection for an asteroid mining mission, Aircraft Engineering and Aerospace Technology 88(3), 458-470.

5 faz.net/aktuell/wissen/weltraum/aufbruch-zu-den-schaetzen-des-alls14512037.html; kurier.at/leben/weltraumrechtler-tagen-in-koeln-ueberschuerfrechte-am-mond-und-weltraumschrott/133.152.323; taz.de/!5534269/; theconversation.com/who-owns-space-us-asteroid-mining-act-is-dangerousand-potentially-illegal-51073. 


\section{Dezentralisierte Kollaborationsplattformen - Alternativen zu globalen Online-Monopolen}

\begin{abstract}
Digitale Plattformen wie Amazon, Google, Facebook, Apple und Microsoft sind die Infrastrukturen des 21. Jahrhunderts, die Handel, Informationsbeschaffung und Vernetzung im globalen Maßstab maßgeblich bestimmen. Ihre Marktmacht ist umfassend und zudem handelt es sich um Unternehmen, die außerhalb der EU angesiedelt sind und damit nur sehr begrenzt auf europäischer oder österreichischer Ebene reguliert werden können. Durch die Erbringung ihrer Dienstleistungen erhalten globale Online-Plattformen zugleich wertvolle Daten, die für die Weiterentwicklung von algorithmischen Entscheidungssystemen (siehe Thema „Transparente Algorithmen") und Künstlicher Intelligenz (KI) (siehe Thema „KIRisiken") von zentraler Bedeutung sind und auch ökonomisch verwertet werden. Die umfassenden Datenbestände, die durch die Nutzung der digitalen Plattformen entstehen, sind eine extrem wertvolle Ressource für die Entwicklung von Märkten und theoretisch auch für die Politik (z.B. zur Identifizierung der Steuerung von umweltrelevanten Entscheidungen). Doch diese Datenbestände stehen weder den BürgerInnen, noch öffentlichen Einrichtungen oder Unternehmen in Europa und Österreich zur Verfügung. Es gibt bereits zunehmend Bedenken gegen die Monopolstellung und es werden verschiedene Maßnahmen diskutiert und umgesetzt, insbesondere im Kartellrecht oder auch über hohe Strafzahlungen bei Verstößen der Unternehmen. Diese bisherigen Versuche, die Marktmacht zu begrenzen, können die monopolistischen Tendenzen nicht grundsätzlich in Frage stellen.
\end{abstract}

Die führenden Plattformen verfügen über ein so hohes Maß an Nutzerdaten und Kapitalreserven, dass sie ihre Geschäftsbereiche jederzeit ausweiten können und jedes Start-Up, das innen entweder gefährlich werden könnte oder aber für ihr eigenes Produktportfolio vielversprechend erscheint, integrieren können. Unternehmen im Bereich Mobilität (Uber) und Beherbergung (Airbnb) zeigen, dass sich auch vormals als regional begriffene Dienstleistungen über globale Plattformen steuern lassen. Wenn der globale Trend auf eine immer umfassendere Plattform-Ökonomie hinausläuft, wird die staatliche Steuerungsfähigkeit der Zukunft davon bestimmt sein, ob die Infrastrukturen durch staatliche Handlungsfähigkeit und demokratische Kontrolle gestaltbar sind (siehe Thema „Digitale Souveränität"). Diese Frage wird immer relevanter, da z.B. Amazon und Facebook Algorithmen und KI einsetzen, die das Verhalten der Nutzerlnnen stark steuern können. Diese Verhaltenssteuerung ist weder transparent, noch erfolgt sie in Einklang mit gesellschaftlichen und politischen Zielen in Demokratien.

Was bisher fehlt, sind Technologien, die darauf ausgerichtet sind, die wesentlichen Funktionen der Ökonomie und des Alltagslebens im 21. Jahrhundert - Handel (siehe Thema „Blockchain"), Informationsbeschaffung, 
Medienzugang - in einer kollaborativen und verteilten Infrastruktur zu organisieren. Globale Netzwerke und Plattformen bestimmen die Zukunft und damit stellt sich die Frage, wie sie im Sinne aller organisiert und transparent gemacht werden können, sowie regionale Unternehmen und Start-Ups unterstützen können. Kollaborative Plattformen im großen Maßstab müssten in der Lage sein, die gleiche Funktionalität der bisherigen Internet-Giganten zu bieten und dabei ein „Ökosystem „von verteilten, interoperablen und kollaborativen Plattformen bilden. Wie ein solches kollaboratives Ökosystem von Dienstleistungsplattformen aussehen kann, welche Technologien dafür bereits zur Verfügung stehen, welche Ansprüche von Politik, Industrie, BürgerInnen, KMUs, Banken und anderen Akteuren dabei berücksichtigt werden müssten und wo noch Forschungsbedarf besteht, ist aktuell noch völlig offen. Die Optionen für solche kollaborativen Plattformen zu untersuchen, würde es ermöglichen, entsprechende Aktivitäten auf europäischer und internationaler Ebene zu initiieren. ${ }^{1}$

1 Erste staatliche Überlegungen in Richtung einer europäischen offenen Kollaborationsplattform bzw. .Europa-Cloud' finden sich gegenwärtig in Deutschland, wo mit der Gründung der Agentur für Sprunginnovationen offene Standards und gemeinsame, europäische Forschungsinfrastrukturen als Alternativen zu turbokapitalistischen Plattformen beworben werden. 


\section{Sicherheits-Robotik}

Der Sicherheitsbereich ist durch das sogenannte Dual-Use-Prinzip gekennzeichnet. Dies bedeutet, dass ursprünglich für den militärischen Einsatz geplante Technologien auch im Bereich der inneren Sicherheit, Kriminalitätsbekämpfung, aber auch anderen zivilen Anwendungen eingesetzt werden. Ein dynamischer Bereich ist in diesem Zusammenhang die Robotik und die Forschung zu Künstlicher Intelligenz (KI). Unbemannte fliegende Systeme (sogenannte Drohnen) sind seit langem fester Bestandteil militärischer Operationen und werden mehr und mehr auch zivil genutzt (siehe Lieferdrohnen, Pandemiemanagement). Drohnen können am einfachsten (teil-)automatisiert werden, da es verhältnismäßig wenig (verschiedene) Hindernisse gibt und sich die (Funk-)Kommunikation relativ einfach gestaltet (TAB 2020).

Aber auch für spezielle Einsätze am Boden (beispielsweise bei der Kampfmittelbeseitigung) oder im bzw. unter Wasser (z. B. zur Seeminenabwehr) kommen bereits unbemannte und teilautomatisierte Systeme zum Einsatz. Die Handlungsautonomie dieser - in Aufbau und Zielsetzung teils sehr unterschiedlichen - Systeme ist derzeit noch beschränkt und ihre Steuerung befindet sich meist regelmäßig unter menschlicher Kontrolle. Gerade stationäre Bodensysteme agieren aber bereits hochautomatisiert (beispielsweise Nahbereichflugabwehr) oder verfügen gar über die technischen Voraussetzungen für einen vollautomatischen Betrieb (Wachpostenkanonen am Gazastreifen und in Südkorea) (TAB 2020).

Angesichts der intensiven Forschungs- und Entwicklungstätigkeit ist zu erwarten, dass der Grad der Autonomie von robotischen und KI-Systemen ansteigen und sowohl ihre militärische als auch ihre nicht-militärische Nutzung deutlich zunehmen wird (TAB 2020). So werden sowohl Robotik und Autonomie, als auch Mensch-Maschinen-Kooperationen vom US Verteidigungsministerium als wichtige langfristige Themen deklariert. ${ }^{1}$

Völkerrechtlich und ethisch ist die rein maschinelle Entscheidungsfindung in Waffensystemen höchst umstritten. Österreich hat sich bereits entschieden und gehört zu den Staaten, die für ein rechtlich bindendes Instrument eintreten, das autonome Waffensysteme ohne ausreichende und effektive menschliche Kontrolle präventiv verbieten würde (BMEIA 2020). Die aufkommende Fragestellung, wie das am besten zu bewerkstelligen ist, ist hochkomplex und tiefergehend zu untersuchen (TAB 2020).

Zudem, da der Sicherheitsbereich oft Grundrechte tangiert, sind grundsätzliche Erwägungen bezüglich Dual-Use (Verschränkung von innerer und äußerer Sicherheit - Polizei und Militär) zu klären. Dazu kommt eine potentiell sehr hohe Zahl (teil-)autonomer Systeme von staatlichen und privaten Betreibern, die nur von speziell ausgebildeten, verantwortungsvollen

1 defensedaily.com/wp-content/uploads/post_attachment/206477.pdf. 
Personen bedient werden dürfen (ITA 2014). In naher Zukunft wird sich auch die Frage der Abhängigkeit von Algorithmen und der damit eingeschränkte Handlungsspielraum von damit befassten Personen ergeben.

\section{Zitierte Quellen}

BMEIA, 2020, Bundesministerium für europäische und internationale Angelegenheiten, bmeia.gv.at/europa-aussenpolitik/abruestung/ konventionelle-waffen/autonome-waffensysteme/.

ITA, 2014, Drohnen - fliegende Alleskönner? ITA-Dossier Nr. 6 (Jänner 2014; Autorin: Julia Haslinger), Wien; epub.oeaw.ac.at/ita/ita-dossiers/itadossier006.pdf.

TAB, 2020, Autonome Waffensysteme, Arbeitsbericht Nr. 187 (Oktober 2020, Autoren: Reinhard Grünwald und Christoph Kehl), Berlin; tab-beim-bundestag.de/de/pdf/publikationen/berichte/TABArbeitsbericht-ab187.pdf. 


\section{Cybersicherheit für kritische Infrastrukturen}

Die Funktionsfähigkeit moderner Gesellschaften ist heute hochgradig von verschiedenen Technologien und deren Zusammenspiel abhängig. Sie bilden dabei „kritische Infrastrukturen“. Diese können als „Hauptschlagader“ von Wirtschaft und Gesellschaft verstanden werden (Strauß/Krieger-Lamina 2017). Dementsprechend bedrohlich sind Ausfälle von Systemen, die zentral für die Funktionsfähigkeit der Daseinsvorsorge und Grundversorgung mit lebensnotwendigen Gütern sind. Neben elektromagnetischen Impulsen (EMP), wie etwa Sonnenstürmen, sind zunehmend Risiken durch gezielte Angriffe auf IT-Systeme (Cyber-Angriffe) von kritischen Infrastrukturen festzustellen (POST 2017; Trimintzios et al. 2017).

Im Hinblick auf die absehbar weiter zunehmende Vernetzung und Automatisierung (z. B. Industrie 4.0, Smart Grids, Smart Home, autonome Fahrzeuge, Internet der Dinge etc.) ist davon auszugehen, dass integrierte Systeme generell weiter an Bedeutung gewinnen werden. Es besteht daher insgesamt Bedarf nach verbesserten Schutzkonzepten von kritischen Infrastrukturen. Mittel- und längerfristig gibt es Bedarf nach Innovationen, die die Systemsicherheit in Design und Architektur insgesamt erhöhen (Securityby-design). Hierbei ist auch ein stärkerer Dialog zwischen Wissenschaft, Wirtschaft und Politik wichtig (vgl. Strauß/Krieger-Lamina 2017).

Das Österreichische Programm zum Schutz kritischer Infrastrukturen $\left(\right.$ APCIP $\left.^{1}\right)$ beinhaltet strategische Maßnahmen, um die Resilienz Österreichs zu erhöhen. Hier wurde bereits einiges geleistet und Österreich zählt hier zu den Vorreitern in der EU. Eine Vielzahl an Strategien und Akteuren widmet sich der Thematik, dies verdeutlicht die Komplexität der Problematik, bringt aber auch Unklarheiten hinsichtlich Kompetenzen und Zuständigkeiten mit sich. Daneben besteht die Österreichische Strategie für Cyber-Sicherheit (ÖSCS) und das staatliche Krisen- und Katastrophenschutzmanagement (SKKM). Eine Analyse (und gegebenenfalls Adaptierung) dieser beiden Elemente in Hinblick auf Überschneidungen, Synergien und Ressourcen mit APCIP und vice versa wäre zweckmäßig (vgl. Strauß/Krieger-Lamina 2017). Im Angesicht der neuesten zunehmenden Angriffe auf österreichische Infrastruktur ${ }^{2}$ werden zwar bereits Maßnahmen unternommen, doch es sollte weiter untersucht werden wie zweckmäßig welche Maßnahmen sind und wie eine sinnvolle Pflichtenverteilung der verschiedenen Akteure erfolgen kann.

\footnotetext{
1 Austrian Programme for Critical Infrastructure Protection.

2 Angriff auf das Außenministerium im Jänner 2020 und Sicherheitslücke im ELAK (fm4.orf.at/stories/3007276/).
} 
Das Parlament als zentraler Ort der politischen Meinungsbildung und Kontrolle kann wesentlich zur Koordination österreichischer staatlicher und privater Aktivitäten sowie zur notwendigen Bewusstseinsbildung in der Öffentlichkeit beitragen. Darüber hinaus wäre durch die Förderung österreichischer Innovationen in technischen und organisatorischen Sicherheitsmaßnahmen doppelter Nutzen zu erzielen.

\section{Zitierte Quellen}

POST (Parliamentary Office of Science and Technology), 2017, Cyber Security of UK Infrastructure, Number 554 May 2017, London; researchbriefings. parliament.uk/ResearchBriefing/Summary/POST$\mathrm{PN}-0554$.

Strauß, S. und Krieger-Lamina, J., 2017, Digitaler Stillstand: Die Verletzlichkeit der digital vernetzten Gesellschaft - Kritische Infrastrukturen und Systemperspektiven. Projekt-Endbericht, 2017-03-31, Wien; epub.oeaw.ac.at/ita/ita-projektberichte/2017-01.pdf.

Trimintzios, P., Chatzichristos, G., Portesi, S., Drogkaris, P., Palkmets, L., Liveri, D., and und Dufkova, A. (STOA), 2017, Cybersecurity in the EU Common Security and Defence Policy (CSDP), Nr. EPRS/STOA/SER/ 16/214N: STOA; europarl.europa.eu/ RegData/etudes/ STUD/2017/603175/EPRS_STU(2017)603175_EN.pdf. 


\section{Cybersicherheit: Vom Dialog zur Ko-Kreation}

\section{Zusammenfassung}

Schon heute werden Angriffe auf Computer und Smartphones zunehmend komplexer und professioneller. Zukünftig nehmen potentielle Angriffspunkte mit der Vernetzung (IoT) und Automatisierung der Produktion, des Verkehrs und der Dienstleistungsbranche sowie der automatisierten Nutzung von Sensoren zu. Cybersicherheit bedeutet die Gewährleistung von Vertraulichkeit, Integrität und Verfügbarkeit in digitalen Infrastrukturen und damit den Schutz von IKT-Systemen (Hardware, Software und zugehörige Infrastruktur), Daten und den bereitgestellten Diensten. Staaten und Unternehmen beobachten zunehmend systematisch die Entwicklung der Cyberkriminalität und entwickeln entsprechende Maßnahmen. ${ }^{1}$ Angesichts der hohen Dynamik digitaler Dienstleistungen, wie Automatisierung von Produktion (Industrie 4.0, Internet der Dinge) und Verkehr, wird Cybersicherheit zunehmend zu einem umfassenden Prozess, der einen hohen Wissensstand nicht nur bei Unternehmen und Betreibern von kritischen Infrastrukturen voraussetzt, sondern auch eine Kultur der Cybersicherheit in allen gesellschaftlichen Bereichen erfordert.

\section{Überblick zum Thema}

Mit der Entwicklung von cyber-physischen Systemen, bei denen informations- und softwaretechnische mit mechanischen Komponenten verbunden sind und der Datentransfer sowie die Steuerung über das Internet in Echtzeit erfolgen, nehmen die Risiken für Störungen durch Cyberbedrohungen stark zu. Cyber-physische Systeme vereinen avancierte Elektronik, fortschrittliche Sensorik und bestehende Infrastrukturen. Sie führen zu einer hohen Abhängigkeit von digitalen Komponenten untereinander ${ }^{2}$ und zu einer Bedrohung dieser Systeme durch Cyberangriffe, die durch das steigende Maß an autonomer Steuerung und global zugänglicher Systeme (z.B. Navigationssysteme) auch ein höheres Bedrohungspotential nach sich ziehen. Das Internet der Dinge (siehe Thema „Netz der bewegten Dinge") trägt durch die zunehmende Integration ungenügend geschützter Geräte im Internet zu neuen Angriffspunkten bei und eröffnet neuartige Angriffsszenarien.

In Österreich sind die "Cyber Sicherheit Plattform" und die Österreichische Strategie für Cyber-Sicherheit darauf ausgerichtet, die gesamtstaat-

1 Vgl. den Bericht Cyber-Sicherheit 2018 und 2019: bundeskanzleramt.gv.at/themen/cyber-sicherheit-egovernment/nis-buero.html?lang=en.

2 Unter anderem durch jeweils unterschiedliche Sicherheitsmerkmale und Kommunikationsstandards, vgl. Burg, et al. (2018). Ein weiteres Problem ist, dass Sicherheit und Gefahrenabwehr in der Ingenieurs- und Informatikbranche traditionell unterschiedliche Probleme sind, die einer Vereinheitlichung bedürfen Wolf/ Serpanos (2018). 
lichen Zusammenarbeit in diesem Bereich zu gewährleisten. ${ }^{3}$ Für aktuelle Gefährdungen der Onlinesicherheit, die BürgerInnen und KonsumentInnen betreffen, gibt es bereits Ansätze, eine breitere Cyber-Sicherheitskultur zu fördern; dazu gehört z.B. das IKT-Sicherheitsportal als interministerielle Initiative in Kooperation mit der österreichischen Wirtschaft, welches aktuelle Themen der Cybersicherheit für breite Kreise bündelt und auf aktuelle Bedrohungen und mögliche Maßnahmen hinweist. ${ }^{4}$

Zugleich entwickeln die EU-Mitgliedstaaten gemeinsame Initiativen weiter. So wird die Einrichtung eines „Kompetenzzentrums für Cybersicherheit in Industrie, Technologie und Forschung" und eines "Netzes nationaler Koordinierungszentren" verhandelt und präzisiert. ${ }^{5}$ Zur Reform der Cybersicherheit in Europa gehören verschiedene Dimensionen:

- die Einrichtung einer wirksamen EU-Agentur für Cybersicherheit6;

- die Einführung eines EU-weiten Zertifizierungssystems für Cybersicherheit;

- die rasche Umsetzung der Richtlinie zur Netz- und Informationssicherheit (NIS-Richtlinie). ${ }^{7}$

Für eine EU-Cyberdiplomatie, die zur Konfliktverhütung, zur Eindämmung von Cyberbedrohungen und zur Förderung der Zusammenarbeit dient, ist eine "Cyber Diplomacy Toolbox" als Teil des EU-Ansatzes geplant. ${ }^{8}$

Die Fragestellungen für die zukünftige Cybersicherheitsforschung zeigen, dass Cybersicherheit zukünftig auf die Nutzerintegration ausgerichtet ist (Jang-Jaccard/Nepal 2014). Damit würde das Thema verstärkt in Politikbereichen aufgegriffen werden müssen, die bisher wenig mit Cybersicherheit verbunden sind. Somit stellen sich Fragen, die auf eine Integration der NutzerInnen verweisen: Hinsichtlich des Datenschutzes geht es darum, Möglichkeiten zu finden, wie InternetnutzerInnen die Vertraulichkeit ihrer personenbezogenen Daten wirksamer schützen und kontrollieren können (Mögliche Ansätze dazu finden sich im Beitrag „Zukunft des Internets: zentral vs. dezentral?"). In Bezug auf ein sicheres Internet der nächsten Generation stellt sich die Frage, wie es möglich ist, das aktuelle InternetSystem von Grund auf neu zu gestalten, ohne dadurch das bestehende System einzuschränken (z. B. Konzepte wie Security-by-Design, oder der Zusammenschluss heterogener Netzwerkumgebungen, Architekturdesign).

3 digitales.oesterreich.gv.at/cyber-sicherheit.

4 onlinesicherheit.gv.at. Vgl. international das Europäische Institut für Telekommunikationsnormen (ETSI), das anerkannte bewährte Verfahren zur Sicherheit loT zusammenfasst, um alle an der loT-Entwicklung beteiligten Parteien mit Hinweisen zur Sicherung ihrer Produkte zu unterstützen. etsi.org/deliver/etsi_ts/ 103600_103699/103645/01.01.01_60/ts_103645v010101p.pdf

5 consilium.europa.eu/de/press/press-releases/2019/03/13/eu-to-pool-and-networkits-cybersecurity-expertise-council-agrees-its-position-on-cybersecurity-centres/.

6 consilium.europa.eu/de/press/press-releases/2019/03/13/eu-to-pool-and-networkits-cybersecurity-expertise-council-agrees-its-position-on-cybersecurity-centres/.

7 consilium.europa.eu/de/policies/cyber-security/.

8 Vgl. Zu aktuellen Entwicklungen: wms.flexious.be/editor/plugins/imagemanager/ content/2140/PDF/2019/pub_9081_responding_cyberattacks.pdf. 
Eine weitere zentrale Frage ist die eines globalen Identitätsmanagements und den damit verbundenen Rückverfolgungstechniken und schließlich die umfassende Frage einer tatsächlich benutzerInnenfreundlichen Sicherheit: Wie lässt sich ein Sicherheitssystem entwickeln, das von BenutzerInnen mit unterschiedlichen Kenntnissen und Ansprüchen tatsächlich verwaltet und kontrolliert werden kann? Alle diese Frage verweisen auf die Herausforderung, dass die etablierten Akteure nicht mehr für Staat, Wirtschaft und Gesellschaft Cybersicherheit entwickeln und implementieren, sondern dass dies zukünftig viel stärker im Zusammenspiel mit den entsprechenden Stakeholdern in vielfältigen Ko-Design-Prozessen geschieht.

\section{Relevanz des Themas für das Parlament und für Österreich}

Digitalisierung bedeutet Komplexität und die Allgegenwärtigkeit der Onlinevernetzung; sie bietet einer immer noch zunehmenden Zahl an Akteuren in Österreich mehr Möglichkeiten im privaten, im wirtschaftlichen, im beruflichen und im öffentlichen Bereich. Digitalisierung bedeutet aber auch eine Zunahme an Cybergefahren, auf die die Akteure vorbereitet sein müssen. Insofern ist Cybersicherheit eine zentrale Voraussetzung für das Gelingen der Digitalisierung und das Vertrauen in Digitalisierungsprozesse.

Mit dem bleibenden Spannungsverhältnis der Gewährleistung von Cybersicherheit, dem Schutz der Privatsphäre und steigendem Datenaustausch im Kontext von Digitalisierung sind zwei Risiken verbunden: Die steigende Gefahr für Staat, Wirtschaft und BürgerInnen, Opfer von Cyberkriminalität und Cyberspionage zu werden, und das Risiko, durch Sicherheitstechnologien in Bürgerrechten eingeschränkt zu werden. Auf grundsätzlicher Ebene stellen sich Fragen nach Freiheit, Anonymität und Handlungsautonomie im Kontext eines hohen Cybersicherheitsniveaus. Diese Fragen zu bearbeiten, wäre die Voraussetzung, um langfristig zu einer breiten gesellschaftlichen Ko-Kreation von Cybersicherheitsprodukten und -dienstleistungen zu kommen.

\section{Vorschlag weiteres Vorgehen}

Das Parlament als zentraler Ort der politischen Meinungsbildung und Kontrolle kann wesentlich zur politikfeldübergreifenden Koordination österreichischer staatlicher, privater und zivilgesellschaftlicher Aktivitäten sowie zur übergreifenden Bewusstseinsbildung zur Cybersicherheit ${ }^{9}$ in der Öf-

\footnotetext{
9 In einem Hearing mit ExpertInnen im Ausschuss für Konsumentenschutz zum Thema Internet of Things am 9.5.2019 wurde das Thema Cybersicherheit als wesentlich für die Entwicklung und Akzeptanz sowie für die zukünftige Konsumentenschutzpolitik im Bereich loT erkannt, siehe parlament.gv.at/PAKT/PR/JAHR 2019/PK0508/index.shtml.

Neben der dort geführten Diskussionen über die Grenzen einer technisch von den Herstellern erzwungen Datenübertragung und der Frage der Regulierung wäre zukünftig auch die Frage zu stellen, welche ganz neue Art von Cybersecurity-Dienstleistungen den KonsumentInnen Services und Tools zur Verfügung stellen, die ihnen eine einfacherer und höhere Souveränität über ihre eigenen Daten / die Daten ihrer Geräte sichert.
} 
fentlichkeit beitragen. Wenn Cybersicherheit zukünftig auf einer breiten Kompetenz in der Gesellschaft basiert, wäre zu identifizieren, was die Anforderungen in verschiedenen Bereichen der Gesellschaft an eine zukünftige Cybersicherheitskultur sind. Aufgrund der steigenden Relevanz des Themas und des Spannungsverhältnisses von Sicherheit, Schutz der Privatsphäre und freiem Datenaustausch wäre eine umfassende, auf Österreich fokussierende Foresight- und TA-Studie mit partizipativen Elementen sinnvoll, die über die Analyse heutiger Cybersicherheit hinaus zukünftige Optionen umfassend antizipiert. Eine transdisziplinäre Studie kann das Wissen von ExpertInnen aus den Bereichen der Sicherheitsdienstleistungen, Landesverteidigung und autonomer Systeme mit Wissensbeständen aus dem Bereich Sicherung der Privatsphäre und KI-Forschung und den divergierenden Interessen unterschiedlicher Stakeholder verbinden, um unterschiedliche Zukunftsoptionen festzustellen. Ziel wäre es, partizipativ Leitlinien für eine Cybersicherheitskultur zu entwerfen und mögliche Innovationspfade zu definieren, die den langfristigen Anforderungen unterschiedlicher Akteure entsprechen und damit auch eine proaktive Cybersicherheitskultur unterstützen.

\section{Zitierte Literatur}

Jang-Jaccard, J. und Nepal, S., 2014, A survey of emerging threats in cybersecurity, Journal of Computer and System Sciences 80(5), 973-993. 


\section{Digitale Schutzengel: Technologien privater Sicherheit}

Mit der Verbreitung von Smartphones steigt die Zahl an Anwendungen (Apps), die auf die Herstellung von privater Sicherheit abzielen. Das sind zum Beispiel Nachhause-Begleit-Apps, ${ }^{1}$ die Personen virtuell nach Hause eskortieren und durch Echtzeitübertragung von Mobilitätsdaten den jeweiligen Standort an ausgewählte FreundInnen oder an andere Nutzerlnnen der App, die sich in der Nähe befinden, kommunizieren. Sogenannte "Schutzengel-Apps" 2 ermöglichen einen raschen Notruf, der entweder durch lauten Schrei, Schütteln des Smartphones, Knopfdruck (auch über Accessoires, wie Ring ${ }^{3}$ oder Schlüsselanhänger) oder Laufen (Bewegungssensoren) ausgelöst werden kann. Viele dieser Apps haben zusätzliche Funktionen, wie z.B. einen Gefahren-Countdown, den der/die NutzerIn nach Ablauf einer bestimmten Zeit deaktivieren muss, um kein Notsignal auszulösen. Manche Apps ermöglichen das Monitoring von Räumen, in denen sich Menschen unwohl fühlen bzw. in denen Gefahren vermutet werden, und stellen dann - anhand der gewonnenen Daten - Karten von risikobehafteten Zonen zur Verfügung und ermöglichen die Vernetzung mit anderen, möglicherweise betroffenen Personen. ${ }^{4}$ Andere Apps zielen in Verbindung mit smarten Gegenständen auf Überwachung und individuellen Schutz, z.B. der intelligente Teppich, ${ }^{5}$ der BewohnerInnen bei Einbruch warnt oder die intelligente Armbanduhr ${ }^{6}$, die Eltern in ihrer Fürsorgepflicht unterstützen soll und das Monitoring der Kinder ermöglicht. Nicht nur Versicherungen investieren in den neuen Markt an Sicherheitstechnologien, auch die Polizei und andere öffentliche Einrichtungen sind an der Infrastruktur privater Sicherheitstechnologien beteiligt.

Wesentliche Beschränkungen der Anwendungen sind zurzeit die Abhängigkeit von GPS-Ortung, WLAN-Empfang bzw. mobiler Internetverbindung und Akku-Kapazität. Sie funktionieren also nur, wenn die Ortung des GPS-Signals möglich ist, was an „Angst-Orten“ wie in Parkhäusern oder in der U-Bahn oft nicht der Fall ist. ${ }^{7}$ Außerdem sollte eine Internetverbindung bestehen, um die Genauigkeit der Ortung zu erhöhen. Zudem wird mit der GPS-Ortung in hohem Maße Akkuleistung verbraucht. Schließlich ist das Angebot der Apps mit der Preisgabe von potentiell sensiblen Da-

1 Z.B. GetHomeSafe, Companion, Wayguard, Watch Over Me, bSafe, u.v.m.

2 Keine Sorgen Schutzengel App, SoftAngel, Mein Notruf App, Vivatar, u.v.m.

3 kickstarter.com/projects/1629204423/nimb-a-smart-ring-that-keeps-you-safeand-sound.

4 dikeapp.de, irks.at/forschung/sicherheitsforschung/topos.html.

5 technologyreview.com/s/429130/a-smart-carpet-to-detect-intruders/.

6 magenta.at/kidswatch/.

7 Eine alternative Ortungsweise, die auch innerhalb von Gebäuden funktioniert, ist die WLAN-basierte Ortung. Eine wesentliche Voraussetzung dafür ist die Verfügbarkeit eines entsprechenden Location-Mapping-Diensts am Endgerät und die uneingeschränkte Nutzung von WLAN-Signalinformationsdatenbanken. 
ten der Nutzerlnnen verbunden: Die Anwendungen erfordern Zugriff auf die Kontakt- und Standortdaten.

Digitale Technologien privater Sicherheit operieren im Spannungsfeld zwischen subjektivem Sicherheitsgefühl (Individualisierung von Sicherheit) und individueller Kontrolle (Privatisierung von Verantwortung). Einerseits können private Sicherheitstechnologien dem Bedürfnis nach subjektiven Schutz- und Sicherheitsgarantien entgegenkommen. Andererseits erzeugen sie neue Erwartungen und Anforderungen im individuellen Umgang mit risikobehafteten Situationen. Gerade in Anbetracht der Vielzahl an Apps und ihrer teilweise spezifischen Ausrichtung auf bestimmte Zielgruppen, wie z.B. solchen, die speziell für Frauen und deren Sicherheitsbedürfnisse entwickelt werden, ist unklar, ob diese privaten Sicherheitstechnologien tatsächlich Risiken minimieren können. Besonders bei staatlichen Angeboten, wie z.B. Fem:HELP, ${ }^{8}$ spielt die Erwartungssicherheit in Hinblick auf ein dauerhaftes und langfristiges Angebot eine große Rolle: Das Beispiel der österreichischen Anwendung, die vom Frauenministerium 2013 vorgestellt wurde, gibt Anlass zur Reflexion, da das Service aktuell nicht mehr verfügbar ist. ${ }^{9}$ Notwendig wäre daher Transparenz hinsichtlich der Wirksamkeit von unterschiedlichen privaten Sicherheitstechnologien, um genauer bestimmen zu können, in welchen Bereichen und unter welchen Umständen diese ein bestimmtes Schutzniveau bieten und wo eben auch die Grenzen privater Sicherheitstechnologien liegen.

8 derstandard.at/story/1378248639399/neue-app-fem-help-soll-in-notsituationenschnell-helfen.

9 aoef.at/index.php/fem-help-app. 


\section{Das Dark-Net}

Mit der Metapher vom „Eisberg“ gesprochen, ist das uns bekannte Internet nur die Spitze. Es handelt sich dabei um das so genannte Clear-Web. Diesen Teil des Internets können wir mittels Suchmaschinen und WebURLs einfach erkunden. Es gibt aber auch einen verborgenen Teil des Internets - das Deep-Web oder versteckte Web. Dabei handelt es sich um Bereiche des Internets welche nicht ohne weiteres durch Web-URLs oder Suchmaschinen erkundet werden können. Das Dark-Net wiederum ist ein eigener Bereich des Deep-Webs, welches unter Zuhilfenahme von speziellen Internet-Browsern erschlossen werden kann (Tor-Services). Das Dark-Net ermöglicht es den NutzerInnen, anonym zu surfen. Diese Anonymität wird sowohl zum Guten als auch zum Schlechten genutzt.

Whistleblower nutzen es als Medium, um die in Ihrem Besitz befindlichen Informationen zu verbreiten und gleichzeitig dabei anonym zu bleiben. Auch investigative Journalistlnnen nutzen das Dark-Net, um an bestimmte, im Clear-Web nicht verfügbare Informationen zu gelangen. Es wird auch genutzt, um in autokratischen Ländern die Zensur zu umgehen. Das Dark-Net bietet einen geschützten Raum der Kommunikation, in dem Vertraulichkeit noch möglich ist. In starken Demokratien gibt es solche Rückzugsorte ohnehin. In Diktaturen hingegen erfüllt das Dark-Net diese Funktion. Durch die zunehmende Tendenz zur Massenüberwachung (auch in westlichen Ländern) schwinden diese Orte.

Das Dark-Net ist aber vermutlich für seine Negativschlagzeilen bekannter. Im Jahre 2011 sorgte es erstmals für Aufsehen, als der erste digitale Schwarzmarkt „eröffnet“ wurde - die "Silk Road“. Es wurden Drogen und verschiedene digitale Güter gehandelt. ${ }^{1}$ Eine Studie aus dem Jahr 2016 wies darauf hin, dass die Präsenz illegaler Inhalte im Dark-Net sehr hoch ist. Sie stellte zudem fest, dass religiös orientierter Extremismus im DarkNet sehr gering war (Moore/Rid 2016). Ihre Erklärung dafür war die eingeschränkte Reichweite des Dark-Nets, was es letztlich für Propagandazecke uninteressant macht. Das Dark-Net ist in gewisser Weise auch ein Einkaufsparadies für Cyberkriminelle, in dem grundsätzlich mittels Kryptowährungen bezahlt wird.

Interpol stärkt mit einem eigenen Programm die internationale Zusammenarbeit im Kampf gegen Cyberkriminalität, die sich im Dark-Net verorten lässt. Im Februar 2020 wurde hierzu eine dreijährige Kooperation mit Südkorea verkündet. ${ }^{2}$ Im Dark-Net erworbene (physische) Güter werden meist über Versanddienstleister zu den Käufern befördert. Dadurch wird der Versanddienstleister unwissentlich zum „Komplizen“. Das Bundesland Hessen hat deshalb Anfang März 2020 einen Gesetzesantrag beim Deut-

\footnotetext{
1 nation.time.com/2013/10/04/a-simple-guide-to-silk-road-the-online-black-marketraided-by-the-fbi/.

2 interpol.int/News-and-Events/News/2020/Korea-to-fund-INTERPOL-projectscombating-cyber-enabled-crime.
} 
schen Bundesrat eingebracht, in dem es darum geht, das Postgesetz zu verschärfen. Damit soll der illegale Handel und Versand mit Betäubungsmitteln und anderen Gütern verhindert werden. Der Gesetzesentwurf sieht vor, dass Beschäftigte von Postdienstleistern dazu verpflichtet wären, verdächtige Sendungen - wobei das nicht näher präzisiert wird - der Polizei zu übergeben. ${ }^{3}$

Das Dark-Net findet auch bei den österreichischen Strafverfolgungsbehörden Beachtung. ${ }^{4}$ Es gibt bereits Anwaltskanzleien, die darauf aufmerksam machen, dass es strafbar ist, illegale Substanzen und Produkte über das Dark-Net zu erwerben. ${ }^{5}$ Denn auch in Österreich sind mittlerweile verstärkt Aktivitäten zu verzeichnen, die mit dem Erwerb illegaler Substanzen über das Dark-Net in Zusammenhang stehen. ${ }^{6}$ Ein österreichisches Start-Up nutzt das Dark-Net als zentralen Bestandteil seines Geschäftsmodells. ${ }^{7}$ Der Service ermöglicht es zu kontrollieren, ob die eigene E-Mail-Adresse, Kreditkartennummer oder sonstige sensible Nutzerlnnendaten im Dark-Net kursieren und verschränkt dabei Dark-Net und Clear-Net.

Chertoff (2017) weist darauf hin, dass ein konstruktiver Diskurs über das Dark-Net notwendig ist. Es gilt zu klären, was dabei die angemessene Rolle des Staates ist und was angemessene Bedingungen für eine Intervention wären. Das zentrale Kriterium für die Funktion des Dark-Nets sind Anonymität und anonyme Rückzugsorte als wichtiger Bestandteil starker Demokratien. Auch für Österreich wäre ein systematischer Diskurs über Potentiale und Probleme des Dark-Nets sinnvoll. Welche möglichen Alternativen für anonyme Rückzugsorte bestehen in einer zunehmend vernetzten Welt und welche Risiken bergen diese (z.B. durch die ungewollte Vereinfachung von Cyberkriminalität)?

\section{Zitierte Quellen}

Chertoff, M., 2017, A public policy perspective of the Dark Web, Journal of Cyber Policy 2(1), 26-38, doi.org/10.1080/23738871.2017.1298643.

Moore, D. und Rid, T., 2016, Cryptopolitik and the Darknet, Survival 58(1), 7-38, doi.org/10.1080/00396338.2016.1142085.

3 bundesrat.de/SharedDocs/drucksachen/2020/0101-0200/10620. pdf?_blob=publicationFile\&v=1.

4 kurier.at/chronik/oesterreich/159-dealer-hinters-licht-gefuehrt/265.476.940.

5 strafverteidiger-wien.at/recht-oesterreich-news/suchtgiftbestellung-imdarknet.html.

6 bmi.gv.at/magazinfiles/2017/07_08/files/drogenbekaempfung.pdf.

7 horizont.at/home/news/detail/intelligence-x-oesterreicher-launcht-erste-darknetsuchmaschine.html. 


\section{Algorithmische Polizeiarbeit}

Unter algorithmischer Polizeiarbeit versteht man die Auswertung von Kriminalitätsdaten und -statistiken, um Voraussagen über zukünftige Delikte treffen zu können. Man spricht in diesem Zusammenhang auch von Predictive Policing (vorausschauender Polizeiarbeit) oder Crime Detection Technologies (Verbrechenserkennungs-Technologien). Basierend auf den Datenauswertungen können Karten erzeugt werden (Crime Maps), in denen Bereiche mit hoher Verbrechenswahrscheinlichkeit gekennzeichnet sind. Das Ziel dieser "Überwachung der Zukunft“ ist es, die Sicherheit präventiv durch z.B. polizeiliche Präsenz in diesen Bereichen zu erhöhen. Da diese Technologie stark auf Mustererkennungsalgorithmen beruht, scheint sie vor allem für Verbrechen geeignet zu sein, die von MehrfachtäterInnen verübt werden, wie zum Beispiel Autodiebstähle oder Einbrüche. Das in vielen Algorithmen zugrundeliegende Prinzip ist die so genannte Near-Repeat Analyse, welche besagt, dass Folgestraftaten öfter in geographischer und zeitlicher Nähe zu einer bereits verübten und früheren Straftat erfolgen.

Zurzeit ist ein verstärkter Einsatz dieser Technologien unter anderem in den USA oder in Deutschland zu beobachten. IBMs BlueCrush, ${ }^{1}$ Pred$\mathrm{Pol}^{2}$ oder PRECOBS, ${ }^{3}$ welches vom Institut für musterbasierte Prognosetechnik in Deutschland entwickelt wurde, stellen erste einsatzfähige Beispiele dieser Technologien dar. Es ist jedoch schwer zu benennen, welchen Erfolg der Einsatz von Predictive Policing tatsächlich bringt, denn wenn die Polizei zu einem mutmaßlich zukünftigen Tatort fährt und dort Präsenz zeigt und kein Verbrechen geschieht, so kann daraus nicht geschlossen werden, dass die „Vorhersage" richtig war.

Eine begleitende Evaluierungsstudie des Max-Planck-Instituts für ausländisches und internationales Strafrecht, attestiert dem Einsatz von PRECOBS, dass die aus den statistischen Modellen errechneten kriminalitätsmindernden Effekte nur moderat sind (Gerstner 2017). Die Prognosegüte der Aussagen hängt stark von der Qualität und Art der Daten ab. Diese erhöht sich, sobald weitere Daten wie beispielsweise Personendaten in der Modellierung berücksichtigt werden, was aus datenschutzrechtlichen Aspekten problematisch ist. Des Weiteren könnte der Einsatz dieser Technologien zur Auflösung der Unschuldsvermutung führen (Legnaro 2015). Befindet sich eine Person gerade in einem Bereich, in dem die Wahrscheinlichkeit für ein Verbrechen hoch bewertet ist, so besteht die Gefahr, dass die Person grundlos unter Verdacht steht. Der breite Einsatz dieser Technologien könnte somit zu Generalverdacht führen. Eine aktuelle Studie zum Einsatz dieser Technologie in Großbritannien kommt des Weiteren zu dem Schluss, dass Predicitive Policing zu sich selbst-

\footnotetext{
1 ibmsystemsmag.com/power/trends/ibmresearch/ibm_research_spss/.

2 predpol.com.

3 ifmpt.de.
} 
erfüllenden Prophezeiungen führen kann. Das heißt, dass gerade die geographischen Areale, in denen aufgrund der Empfehlung des Algorithmus vermehrt polizeiliche Präsenz vorherrscht, auch mehr Verbrechen registriert werden. Das führt möglicherweise zu einem Vertrauensverlust in der Beziehung zwischen BewohnerInnen dieser Areale und der Polizei. Die Autorlnnen der Studie empfehlen zum einen, dass die PredicitivePolicing-Programme in Großbritannien in der Form, in der sie zurzeit stattfinden, nicht mehr fortgeführt werden sollten. Des Weiteren sollte vermehrt daran gearbeitet werden, die in den Predicitive-PolicingAlgorithmen verpackten und potentiell diskriminierenden Parameter zu identifizieren und zu korrigieren. Jeglicher Einsatz dieser noch relativ jungen Technologie sollte von externen, interdisziplinären ExpertInnen begleitet und evaluiert werden. Grundlegend sollte hierbei das Ziel verfolgt werden, die langsame Unterminierung des Justizsystems mit fehlerbehafteten Algorithmen, zu verhindern. ${ }^{4}$

Da Predictive Policing auch in Österreich von der Abteilung Kriminalanalyse des Bundeskriminalamtes bereits seit geraumer Zeit genutzt wird und die Nutzung auch weiter ausgebaut werden soll, ${ }^{5}$ könnte sich eine interdisziplinäre Vertiefungsstudie mit der Einordnung der österreichischen Praktiken in der internationalen Landschaft beschäftigen. Besonderes Augenmerk sollte dabei auf die zuvor genannten und bereits international vielfach erkannten Probleme gelegt werden, um zu verhindern, dass potentiell fehlerbehaftete, diskriminierende und undurchsichtige Algorithmen das Justizsystem durchdingen.

\section{Zitierte Quellen}

Gerstner, D., 2017, Predictive Policing als Instrument zur Prävention von Wohnungseinbruchdiebstahl: Evaluationsergebnisse zum BadenWürttembergischen Pilotprojekt P4, Freiburg im Breisgau Germany; mpicc.de/files/pdf4/rib_50_gerstner_2017.pdf.

Legnaro, A. K., Andrea 2015, Das Polizieren der Zukunft, Kriminologisches Journal (2), 94-111;

beltz.de/fachmedien/erziehungs_und_sozialwissenschaften/zeitschrifte n/kriminologisches_journal/article/Journal.html?tx_beltz_journal\%5Bart icle\%5D=30428\&cHash=2513c21b829f6cace61600380c551e3b.

4 libertyhumanrights.org.uk/policy/report-policing-machine-policing-machine.

5 jku.at/kepler-tribune/artikel/vorzeitige-verhaftung/at/keplertribune/artikel/vorzeitige-verhaftung/. 


\section{Fortgeschrittene Gesichtserkennung}

Neben spezialisierten Entwicklungsfirmen aus dem Sicherheitsbereich arbeiten auch alle großen Internet-Konzerne an der Weiterentwicklung von Gesichtserkennungs-Software. Dabei wird zunehmend Künstliche Intelligenz verwendet, um die Bilderkennungsraten zu verbessern. Derzeit liegen die Erkennungsraten bei Bildern aus bestehenden Datenbanken abhängig von deren Qualität - bei maximal $80 \%$. Das heißt von 100 analysierten Bildern wurden 80 einem anderen Bild einer Person richtig zugeordnet. Bei Bildern aus Überwachungsanlagen hingegen ist man derzeit noch weit davon entfernt. Hier liegt im Gegensatz dazu die Rate der falsch-positiven Zuordnungen bei bis zu $90 \%{ }^{2}$ Möglich werden die Verbesserungen vor allem durch den unglaublich großen Bestand von Bildern im Netz, an denen die Software "trainiert" werden kann. So hat Facebook neben dem Eigenbestand von Bildern seiner etwa 2,2 Milliarden Userlnnen auch Zugriff auf die Datenbank des bildorientierten SocialMedia-Kanals Instagram. Die dort verfügbaren 3,5 Milliarden „öffentlichen“ Fotos, die unter 17.000 Hashtags gefasst waren, wurden, ohne die NutzerInnen darauf hinzuweisen, ebenfalls einer Analyse unterzogen und dienten so zur Erhöhung der Treffergenauigkeit. ${ }^{1}$ Ziel dabei ist es unter anderem, den Komfort für die NutzerInnen zu erhöhen und den Prozess der Beschriftung oder "Markierung“ von Freunden und Bekannten, die auf im Netzwerk veröffentlichten Fotos erscheinen, zu beschleunigen.

Bereits heute wird Software angeboten, die in der Lage ist, Personen von einem aufgenommenen Foto zu identifizieren, so diese in einer zugrundeliegenden Datenbank vorhanden sind. Ein wesentlicher Sprung in der Genauigkeit wird erwartet, sobald 3D-Bilder von Gesichtern verfügbar sein werden (Kuusi and Vasamo 2014). Zudem sind für die nahe Zukunft auch Fortschritte in der Verbindung von Informations- und Kommunikationstechnologien und genetischen Informationen absehbar, die wahrscheinliche Bilder von Gesichtern konstruieren können sollen. Wenn genetische Informationen aus einer menschlichen Zelle mit einer umfangreichen Gesichtserkennungsdatenbank kombiniert werden, kann diese Art von Software mögliche Gesichter der Person, zu der die Zelle gehört, vorschlagen. ${ }^{2}$ Der umgekehrte Fall, aus zwei Bildern auf genetische Verwandtschaft bzw. Zugehörigkeit zu schließen, wird bereits heute angeboten. ${ }^{3}$ Diese Entwicklungen lassen Anwendungen im Sicherheits- und Überwachungsbereich entstehen, die die Detektion eines Menschen aufgrund von DNA-Spuren aus einer Menge heraus möglich machen werden. Dies führt insbesondere dazu, dass kritisch hinterfragt werden sollte,

\footnotetext{
1 futurezone.at/digital-life/facebook-analysierte-35-milliarden-instagram-fotosohne-wissen-der-user/400031245.

2 theconversation.com/dna-facial-prediction-could-make-protecting-your-privacymore-difficult-94740.

3 faceitdna.com.
} 
ob durch derzeitige Methoden der Anonymisierung genetischer Daten die Anonymität tatsächlich aufrechterhalten werden kann (siehe Thema „Digitalisierung und Anonymität“). Für das Parlament stellt sich die Frage, wie mit neuen Technologien umgegangen werden soll. Wie können Bürgerlnnen vor Missbrauch durch private Nutzerlnnen geschützt werden? Und wie soll im Angesicht versprochener sicherheitspolitischer Zugewinne und dem demokratiepolitisch notwendigen und grundrechtlichen zugesicherten Recht auf Privatsphäre der Einsatz derartiger Technologien im Bereich der inneren Sicherheit geregelt werden?

\section{Zitierte Quellen}

Kuusi, O. und A.-L. Vasamo (2014). 100 opportunities for Finland and the world. Helsinki, Committee for the Future. 


\section{Authentifizierung durch Verhalten}

Die Authentifizierung von Internet-NutzerInnen ist vor allem für Finanztransaktionen, Vertragsabschlüsse oder sichere Zugänge zu OnlineKonten wichtig. Mittlerweile ist diese Authentifizierung auch durch die Analyse des Verhaltens von NutzerInnen möglich. Konkret geht es um die Art und Weise, wie z.B. getippt wird (typischer Tastaturanschlag) und wie Smartphone-NutzerInnen ihr Gerät während der Eingabe halten. Aus der unterschiedlichen Dauer und Geschwindigkeit eines Tastendrucks lässt sich über eine kurze Zeitspanne ein individuelles Profil erstellen. Die Verfügbarkeit dieser persönlichen Daten ist aufgrund der zahlreichen eingebauten Sensoren gegeben. Die Authentifizierung mittels NutzerlnnenVerhalten läuft oft im Hintergrund herkömmlicher Login-Varianten und wird von Banken bereits kommerziell genutzt. ${ }^{1}$ Neben Finanzdienstleistern sind Universitäten, E-Learning-Provider, Anwaltskanzleien, OnlineServices und viele weitere Branchen Zielgruppe der Technologie.

Bisher wurden dafür individuelle Passwörter genutzt, die je nach gewählter Länge und Komplexität sicher oder unsicher sind. Auch MultifaktorLogins anhand von Hardware-Tokens, Bankkarten oder Schlüssel in Kombination mit Einmalkennwörtern, PINs oder TANs sind übliche Authentifizierungsvarianten. Biometrische Merkmale werden immer öfter für die eindeutige Erkennung von Internet-Nutzerlnnen herangezogen. Dazu zählen Fingerabdrücke, Muster der Regenbogenhaut (Iris-Erkennung), Gesichtserkennung (siehe Thema "Fortgeschrittene Gesichtserkennung") oder Stimmprofile. Diese bisherigen Authentifizierungsmethoden sind durch bewusste und durch die Individuen steuerbare Handlungen bestimmt. Die Analyse des NutzerInnenverhaltens kann dagegen im Hintergrund geschehen.

Nicht nur private Unternehmen haben Interesse an der neuen Authentifizierungs-Technologie, sondern auch die Forschungsbehörde des USamerikanischen Militärs (DARPA) finanziert die Entwicklung von verhaltensanalytischen Technologien. ${ }^{2}$ Gerade in diesem Kontext drängt sich die Frage auf, wie persönliche Daten, die durch Verhaltensauthentifizierung gesammelt werden, weiterverarbeitet werden. Mit dem Argument der Cybersicherheit könnten Staaten die Technologie nutzen, die andere Prinzipien anlegen, als es im europäischen Rechtsrahmen vereinbart ist. Auch Werbetreibenden stünden Daten zur Verfügung, die ohne Wissen und Zustimmung der Kundlnnen gesammelt werden können und mit denen diese durch das gesamte Internet verfolgt werden könnten.

Obwohl bereits Software existiert, ${ }^{3}$ die auf die Verschleierung von Verhaltensdaten abzielt (indem Eingaben um einige Millisekunden verzögert oder beschleunigt werden), bleibt die Frage der transparenten Anwendung

\footnotetext{
1 behaviosec.com/danske-bank-deploys-behaviosec/.

2 opencatalog.darpa.mil/AA.html.

3 chrome.google.com/webstore/detail/keyboardprivacy/aoeboeflhhnobfjkafamelopfeojdohk.
} 
von Verhaltensanalysetools offen. Hier könnte das österreichische Parlament mit gesellschaftlich verhandelten Transparenzrichtlinien bei der Generierung und Verarbeitung von verhaltensbezogenen Daten gestalterisch auf die Zukunft einwirken. 


\section{Existenzielle Risiken von Künstlicher Intelligenz}

Die Entwicklung von Künstlicher Intelligenz (KI) wird gegenwärtig umfassend vorangetrieben und feiert insbesondere Erfolge bei Spielen: Nachdem Computer im Schachspiel schon lange gewonnen haben, besiegt 2016 die von Google entwickelte künstliche Intelligenz AlphaGo den mehrfachen Weltmeister Lee Sedol im komplexen Go-Spiel und 2017 gewinnt die Software Libratus gegen vier der weltbesten Pokerspieler. Poker war insofern eine Überraschung, als erfolgreichen Spielerlnnen ein hohes Maß an Intuition und psychologischem Geschick zugeschrieben wird. Selbst in Fernseh-Quizsendungen, in denen es darum geht, Wörter und deren Kontext zu analysieren, können Computer Menschen besiegen. ${ }^{1}$ Führende Internetkonzerne wie Amazon, Apple, Microsoft, Facebook und Google investieren Milliarden in die KI-Forschung. Es ist nicht nur hinsichtlich der Forschungsgelder abzusehen, dass künstliche Systeme immer mehr lernen, den Systemen stehen auch immer mehr Daten und Experimentierfelder zur Verfügung (mit jedem neuen Dienst, den die Plattformen einführen).

Offen sind drei zentrale Fragen: Die Frage danach, was KI-Systeme in wenigen Jahren können werden, was sie wollen, also welchen Zielhorizonten sie folgen werden, und schließlich die Frage, was dies mit den Menschen und der Gesellschaft machen wird.

Wesentlich für die komplexen und weitgehend unvorhersehbaren Entwicklungspfade ist das „Deep Learning“, das die KI-Forschung stark verändert hat. Hierbei werden Informationen in künstlichen neuronalen Netzen verarbeitet, die analog zum menschlichen Gehirn in hierarchischen Stufen angeordnet sind und damit ein "tiefergehendes Lernen" ermöglichen sollen. Die starke Unvorhersehbarkeit liegt darin, dass diese KISysteme aus den gigantischen Datensätzen selbstständig Konzepte und Weltmodelle entwickeln, was oft in Analogie zum Lernen von Kindern dargestellt wird. Mit dem exponentiellen Wachstum von Daten und Rechnerkapazität kann damit eine Komplexität und Geschwindigkeit an Lernfähigkeit in wenigen Jahren erreicht werden, die die individuelle und politische Kontrolle dieser Systeme als unmöglich erscheinen lässt.

Im Feuilleton steht oft die Frage im Vordergrund, was das originär Menschliche der menschlichen Intelligenz ausmacht, wenn KIs komplexe Aufgaben in hoher Geschwindigkeit lösen werden. ${ }^{2}$ Gleichzeitig ist die relevante Frage auf politischer Ebene die nach der Steuerung von Prozessen im Kontext der Unberechenbarkeit zukünftiger KI-Fähigkeiten.

\footnotetext{
1 futurezone.at/science/computer-watson-siegte-bei-jeopardy/24.563.160.

2 zeit.de/2018/14/kuenstliche-intelligenz-menschen-maschineverhaeltnis/komplettansicht.
} 
Diese Unvorhersehbarkeit zu konkretisieren, bedeutet zugleich, die „ontologische Expansion“ (Tuomi 2012) zur berücksichtigen: Mit KI können künstliche Weltmodelle entstehen, die ganz anders als unsere bekannten Weltmodelle sind, aber auch zu anderen Handlungsstrategien von Künstlichen Intelligenzen führen können.

\section{Zitierte Quellen}

Tuomi, I., 2012, Foresight in an unpredictable world, Technology Analysis \& Strategic Management 24(8), 735-751. 


\section{CO2 als Ressource}

Die chemische Industrie produziert viele kohlenstoffbasierte Produkte als Zulieferer für andere Industriezweige und verwendet dabei weitestgehend fossile Rohstoffe wie Erdöl. Könnten diese durch $\mathrm{CO}_{2}$ aus der Atmosphäre oder aus Abgasen von Großindustrien wie der Zement-, Stahl- oder Biogasproduktion ersetzt werden, wäre das ein wichtiger Schritt in Richtung Kreislaufwirtschaft und nachhaltige Entwicklung.

Die Abscheidung und Speicherung von $\mathrm{CO}_{2}$ beispielsweise in Tiefengesteinsschichten wurde bisher überwiegend im Energieerzeugungsbereich (Carbon Capture and Sequestration (CCS)) diskutiert. Die gasförmige Speicherung birgt jedoch hohe Risiken für Umwelt und Gesellschaft vor allem da es wenige langfristig sichere Lagerstätten gibt (IPCC 2018). In einer Pilotstudie in Island wird abgeschiedenes $\mathrm{CO}_{2}$ unterirdisch in basalthaltigem Grundwasser zu Carbonatgestein mineralisiert, und so längerfristig gebunden (Pogge von Strandmann et al. 2019). Auch die großflächige Ausbringung von $\mathrm{CO}_{2}$-bindenden Materialien, z. B. in Form von grünem Sand an Stränden, wird diskutiert. ${ }^{1}$ Derzeit ist die Abscheidung von $\mathrm{CO}_{2}$ aus Luft oder Abgasen aufwendig und teuer. Für die weitere chemische Nutzung werden verschiedene Konzepte mit Hilfe staatlicher Förderung beforscht. ${ }^{2}$

Die Nutzung von $\mathrm{CO}_{2}$ als Ressource für kohlenstoffhaltige Materialien etwa für die Auto-, Textil-, und Pharmaindustrie oder auch biotechnologische Herstellung von Treibstoffen wie Ethylen könnte zumindest helfen $\mathrm{CO}_{2-}$ Emissionen in der Industrie zu senken, wenn dadurch fossile Rohstoffe ersetzt würden. Eine klimaschutzrelevantere längerfristige Entfernung von $\mathrm{CO}_{2}$ aus der Atmosphäre könnte etwa durch Mineralisierung zu Baustoffen erreicht werden (Hepburn et al. 2019).

Insgesamt entstehen gerade erste Geschäftsmodelle, die auch langsam von der Industrie gefördert werden. ${ }^{3}$ Finanzierung und Management des Entstehens einer neuen Reihe von Industrien zur $\mathrm{CO}_{2}$-Nutzung wird aber wahrscheinlich eine klare Richtung durch den Gesetzgeber erfordern (Hepburn et al. 2019).

\section{Zitierte Literatur}

Hepburn, C., et al., 2019, The technological and economic prospects for $\mathrm{CO}_{2}$ utilization and removal, Nature 575(7781), 87-97, doi.org/10.1038/s41586-019-1681-6.

\footnotetext{
1 technologyreview.com/2020/06/22/1004218/how-green-sand-could-capturebillions-of-tons-of-carbon-dioxide/.

2 chemieundco2.de/uploads/CO2plus_Abschlussergebnisse.pdf.

3 co2-utilization.net/de/.
} 
IPCC, 2018, Global Warming of $1.5^{\circ} \mathrm{C}$. An IPCC Special Report on the impacts of global warming of $1.5^{\circ} \mathrm{C}$ above pre-industrial levels and related global greenhouse gas emission pathways, in the context of strengthening the global response to the threat of climate change, sustainable development, and efforts to eradicate poverty., im Auftrag von: Masson-Delmotte, V., et al. (eds.).

Pogge von Strandmann, et al, 2019, Rapid $\mathrm{CO}_{2}$ mineralisation into calcite at the CarbFix storage site quantified using calcium isotopes, Nature Communications 10(1), 1983 doi.org/10.1038/s41467-019-10003-8. 


\section{Zukunft der Batterieentsorgung: Wohin mit ausgebrannten Elektroautos?}

Was tun, wenn ein Elektroauto in Brand gerät? Wie werden ausgebrannte Elektroautos entsorgt? Das sind Fragen, die mit einer Zunahme von Elektroautos und möglichen Brandunfällen in Zukunft an Bedeutung gewinnen werden. Die Brandbekämpfung bei Elektroautos ist aufgrund der chemischen Reaktionen, die in einer Batterie im Brandfall ablaufen, schwieriger als bei herkömmlichen Verbrennern; außerdem ist es schwer festzustellen, wann die Reaktion definitiv zu Ende ist und kein Wiederaufflammen mehr eintreten kann. Daher werden brennende E-Autos für längere Zeit in Kühlwassercontainern versenkt oder mit Sand überschüttet, um den Brand nachhaltig zu stoppen. Eine größere Herausforderung entsteht aber erst nach der Brandlöschung: das Beispiel des ausgebrannten E-Autos, das 2019 österreichische Expertlnnen beschäftigte, verdeutlicht, dass nicht die Brandbekämpfung an sich, sondern die darauffolgende Entsorgung, v. a. der beim Batteriebrand entstehenden chemischen Fraktionen, Probleme bereitet. ${ }^{1}$ Die Rochaden um die Frage, wie das E-Autowrack entsorgt werden sollte, dauerten über Wochen nach dem Unfall an (siehe Smarte Straßen).

In Österreich wird bereits an Risiken und Auswirkungen von Batterien in abfallwirtschaftlichen Systemen geforscht ${ }^{2}$, auch Sicherheitstests und Forschung an zukünftigen Batterietechnologien zur sicheren Integration in EFahrzeuge finden statt. ${ }^{3}$ Der finanzielle Schaden, der der Öffentlichkeit durch unsachgemäße Entsorgung von Lithium-Batterien entsteht, wird mit steigender Anzahl batteriebetriebener Geräte weiterhin zunehmen. ${ }^{4}$ In Anbetracht der zahlreichen politischen Strategien zur Förderung der EMobilität und Digitalisierung scheint es ratsam zu überlegen, wie politische Verantwortung für die Entsorgung - teils in der Anschaffung geförderter - batteriebetriebener Produkte übernommen werden kann. Anzudenken wäre bspw. die Deklaration von Herstellungskomponenten durch QR-Codes bzw. die Nachverfolgung der in Umlauf gebrachten Batterien durch RFID-Tags (siehe Lebensmitteltracking), was die Entsorgung erheblich erleichtern könnte. Gleichzeitig wären verstärkte Maßnahmen zur Bewusstseinsbildung bei der Batterieentsorgung wünschenswert. Wie Batterie-Recycling zukünftig gestaltet werden kann und die externen Kosten der Entsorgung von Lithium-Batterien zukünftig gesenkt bzw. fair verteilt werden können, wären relevante Fragen für einen breit angelegten Foresight- und TA-Prozess.

\footnotetext{
1 teslamag.de/news/ausgebrannter-tesla-bereitet-in-oesterreichentsorgungsprobleme-25735.

2 avaw-unileoben.at/de/forschung-ag_future_waste_treatment-laufende_projektebat-safe/.

3 ait.ac.at/themen/battery-technologies/.

4 ecyclingportal.eu/Archive/44523; voeb.at/service/voeb-blog/detail/showarticle/steigende-brandgefahr-durch-lithium-batterien/.
} 


\section{Lichtverschmutzung}

\section{Zusammenfassung}

Zumindest die Hälfte der Europäer sieht nachts die Milchstraße nicht und die meisten wundern sich darüber auch nicht (mehr). Neue Lichttechnologien, die zunehmende Urbanisierung und weitere Faktoren sorgen dafür, dass die Abstrahlung unserer vielfältigen Lichtquellen auch nachts ein unnatürlich hohes, konstantes Helligkeitslevel erzeugt. Diese als "Lichtverschmutzung“ bezeichneten Faktoren haben vielfältige Einflüsse: So kann ein zu hohes Helligkeitslevel beim Menschen zu Schlafproblemen führen, es stört ökologische Prozesse und nicht zuletzt wird professionelle und Laien-Astronomie immer schwieriger. Gleichzeitig gibt es immer mehr Hilfsmittel um einzuschlafen, z. B. Blaulichtfilter bei Bildschirmen oder Brillen, deren Wirksamkeit aber wissenschaftlich nicht erwiesen ist. Bezüglich des Helligkeitslevels außerhalb des Schlafzimmers, lässt sich durch die immer weiter verbreitete Nutzung von LED als Lichtquelle Richtung und Intensität des Lichts immer besser steuern. Hierdurch können einerseits Energie gespart und Kosten reduziert und andererseits den oben genannten Prozessen entgegengewirkt werden.

\section{Überblick zum Thema}

Der Nachhimmel in Europa ist zu fast 100 \% von künstlicher Beleuchtung beeinflusst (TAB 2020). Von den meisten Orten aus ist die Milchstraße zwar noch sichtbar, das gilt aber nur für eine Minderheit der Bevölkerung. An sehr wenigen Orten ist es so hell, dass das Auge sich nicht mehr an Dunkelheit anpassen muss, das betrifft allerdings ein Fünftel der EuropäerInnen. Wirklich unbeleuchtete Orte sind selten geworden (ebd.). Die Lichtkegel von Städten strahlen noch über $100 \mathrm{~km}$ weit. Straßen, kommerziell oder öffentlich genutzten Flächen, Flughäfen und Industrielle Anlagen sind insgesamt für große Anteile der Lichtemissionen verantwortlich (ebd.).

Auch die weitverbreitete Einführung von lichtemittierenden Dioden (LED) hatte und hat eine Reihe von Folgen. LED verbrauchen um ein vielfaches weniger Strom als herkömmliche Leuchtmittel und sind damit weniger energie- und somit kostenintensiv. In einem Rebound-Effekt werden gerade private Beleuchtungen dadurch oft immer weiter ausgebaut und es wird weniger darauf geachtet, wo und zu welchen Betriebszeiten sie auch sinnvoll sind.

Auch strahlen LED in einem Licht, das mehr Blauanteile enthält. Dieses wird vom menschlichen Auge als wesentlich heller wahrgenommen. Das ist einer der Gründe weswegen es im Verdacht steht für Mensch und Natur nachts besonders störend zu sein. Dass LED Licht als heller wahrgenommen wird, ist wiederum auch ein Vorteil, da so gewünschte Helligkeiten mit niedrigerem Material- und Energieaufwand erreicht werden können. Zudem ist die Beleuchtung durch LED sehr gut ausrichtbar und kann
Wenig natürlich dunkle Orte in Europa übrig

Lichtemittierende Dioden [LED] erzeugen einen Rebound-Effekt

Höhere Blauanteile in LED Licht 
Einfluss künstlichen

Lichts auf den

menschlichen

Schlafzyklus

Blaulicht-Filter für Bildschirme

weitere SchlafOptimierungs-Hilfen sehr exakt Objekte beleuchten und das Umfeld unbeschienen lassen. Oft werden aber nur Leuchtmittel ausgetaucht und keine Anpassung des gesamten Beleuchtungskonzepts vorgenommen, wodurch diese Potentiale ungenutzt bleiben (TAB 2020). Im schlechtesten Fall führen neue LED in alten Fassungen zu einer Verschlechterung der Beleuchtungssituation, etwa weil sie Blendung erzeugen (ebd.).

Da die innere Uhr des menschlichen Körpers auf einen etwa 25-stündigen Rhythmus eingestellt ist, muss diese immer wieder durch den natürlichen Tag-Nacht Zyklus angepasst werden (Rodenbeck 2020). Die Nutzung von künstlichem Licht, wie sie in unserer Zivilisation normal ist, kann dieses Rücksetzen des Schlafrhythmus erschweren. Dies kann zu Müdigkeit während der Tageszeit führen, welche mit einer Reihe unerwünschter Folgen assoziiert wird: erhöhte Gefährdung im Straßenverkehr, verringerte Produktivität und allgemein schlechtem Befinden bis hin zu Depressionen. Im schlimmsten Fall können solche Schlaf-Rhythmus-Störungen zu chronischem Schlafmangel führen, der mit einer Reihe zusätzlicher Gesundheitsrisiken einhergeht (Rodenbeck 2020). Helligkeit und insbesondere blaues Licht hemmen die Produktion des Hormons Melatonin. Dieses wiederum ist hauptverantwortlich für die Regulation des Schlafes, dessen Tiefe und von Müdigkeit allgemein.

Obgleich es Studien gibt, die einen Zusammenhang andeuten, kann wissenschaftlich nicht zweifelsfrei nachgewiesen werden, dass blaues Licht zu Schlafproblemen führt (TAB 2020). Dennoch sind viele Privatpersonen besorgt über den Einfluss blauen Display-Lichts auf ihre Schlafqualität und installieren Filter, die durch die Ausfilterung der Blauanteile das Display weniger grell und damit angenehmer erscheinen lassen sollen. ${ }^{1}$ Diesen Trend haben auch die Hersteller erkannt, und inzwischen haben alle gängigen Betriebssysteme Filter und/oder Nachtmodi integriert. Letztendlich wird Wachheit aber eher subjektiv empfunden, wodurch sich die Frage stellt, ob nicht andere Faktoren mehr Einfluss haben: So wäre ein gewisser Placebo-Effekt denkbar, besonders wenn der Blaufilter zeitgesteuert mit dem Sonnenuntergang einsetzt und so unterschwellig das Ende des Tages anzeigt. Auch geht dadurch möglicherweise die Mediennutzung später am Abend zurück, die beim Konsum mehr oder weniger aufwühlender Inhalte zu Einschlafproblemen führen könnte. Letztendlich ist also gerade bei elektronischen Displays fraglich, ob die Reduzierung der Blauanteile des ausgestrahlten Lichts tatsächlich schlafverbessernd wirkt. Fakt ist, dass im Rahmen der Selbstoptimierungsbewegung auch das persönliche Schlaferlebnis zu optimieren versucht wird - so gibt es Getränke, die nach eigenen Angaben mit ausschließlich natürlichen Kräutern und Wirkstoffen beim Einschlafen helfen sollen ${ }^{2}$ sowie Apps, die die Schlafphase erkennen sollen und dementsprechend die Weckzeit steuern. Letztere nutzen meist entweder die Vibration der Matratze oder die Bewegungsdaten

\footnotetext{
1 derstandard.at/story/2000107711402/handy-displays-ist-blaues-licht-einschlafraeuber-oder-harmloser-reiz.

2 derstandard.at/story/2000118814282/schlafoptimierung-an-den-hebeln-dernacht.
} 
von sogenannten Fitness-Armbändern oder Smartwatches als Grundlage, die dann per Bluetooth an das Smartphone übertragen werden. Die hierbei gesammelten Daten werden analysiert und Empfehlungen gegeben. Während zumindest teilweise gesundheitsförderliche Ratschläge gegeben werden, ist die Datensicherheit teils fragwürdig (siehe Datengetriebene Medizin).

Außerordentlich sind die Einflüsse unnatürlichen Lichts für manche Tierarten. Besonders Insekten, aber auch Vögel, Fledermäuse, Fische und einige Arten von Säugetieren werden in ihrem Tag-Nacht-Rhythmus gestört und leiden somit unter hormonellen Veränderungen und verminderten Reproduktionsraten (TAB 2020). So kreisen manche Insekten beispielsweise bis zur Erschöpfung um Straßenlaternen und pflanzen sich infolgedessen nicht mehr oder seltener fort. Dies ist somit auch ein Faktor des fortschreitenden Insektensterbens, das die Artenvielfalt bedroht - so stirbt auch die Nahrungsgrundlage für viele weitere Tierarten, besonders Vögel - und letztendlich zu ökonomischen Problemen führt. ${ }^{3}$

Leider ist es sehr schwer, da sehr komplex, Grenzwerte für Beleuchtungsstärke und -zeiten zu bestimmen, die für die unterschiedlichen Tierarten noch akzeptabel sind. Somit sind handfeste wissenschaftliche Erkenntnisse vergleichsweise rar. Als gegeben gilt jedoch, dass blaues Licht wesentlich mehr Insekten anzieht (Posch 2013) und dass herkömmliche LED einen höheren Blauanteil ausstrahlen. Mittlerweile gibt es jedoch besonders warmweiße LED, die besonders wenig nachtaktive Insekten anziehen (Eisenbeis und Eick 2011).

\section{Relevanz des Themas für das Parlament und für Österreich}

Der offensichtlichste Effekt von Lichtverschmutzung ist der Energieverbrauch. Durch LED-Technologie ist dieser zwar vergleichsweise gering, jedoch könnte durch eine Optimierung der Strahlrichtung, Intensität und Betriebszeit vieler Beleuchtungen Energie gespart werden. Dies beginnt im öffentlichen Raum bei der Straßenbeleuchtung und der Bestrahlung von Wahrzeichen und kann in den industriellen, gewerblichen und privaten Raum weitergetragen werden. So dürfen beispielsweise in Frankreich nachts Schaufenster nicht mehr beleuchtet werden.

Zum Thema Schlafgesundheit gibt fast die Hälfte der ÖsterreicherInnen an, nicht genug zu schlafen und fast ein Drittel hat Einschlafprobleme (Blume et al. 2020). Letztere sind im Vergleich zu vor zehn Jahren um ein Vielfaches gestiegen (ebd.). Hierbei gibt es den Verdacht, dass die zunehmende nächtliche Helligkeit ein möglicher Einfluss ist - jedoch sind andere Faktoren wie Stress, Ernährung und Bewegung wesentlich einflussreicher. Auch ein direkter Zusammenhang zwischen Helligkeit vor dem Fenster und in der Wohnung kann bislang nicht belegt werden (TAB 2020).
Negative Einflüsse nächtlicher Beleuchtung auf gerade nachtaktive Tierarten

Schwierige wissenschaftliche Grundlage, um Grenzwerte zu bestimmen

Energieeinsparungspotential

Einschlafprobleme zunehmend verbreitet in Österreich 3 Für mehr Informationen zum Einfluss des Insektensterbens in Österreich siehe
zobodat.at/pdf/ENTAU_0027_0269-0283.pdf. 
Astrotourismus als Chance für Österreich

Interesse verschiedener Bevölkerungsgruppen am Thema

Reduzierte

Straßenbeleuchtung in Städten bleibt meist unbemerkt

Beispiel Slowenien für nationale Gesetzgebung
Gerade für Österreich als Tourismusstandort hat Lichtverschmutzung aber auch ökonomische Folgen. Im sogenannten Astrotourismus ist Österreich teils internationaler Vorreiter. ${ }^{4}$ Es gibt in Österreich bereits dedizierte Lichtschutzgebiete, doch auch von dort sieht man meist das Himmelsleuchten teils weit entfernter Siedlungen. International gibt es nur rund 80 zertifizierte „Parks mit dunklem Himmel“5, bislang ist zwar keiner davon in Österreich, aber es gibt bereits zwei Anwärter darauf. 6

Generell setzen sich verschiedene Akteure bereits intensiv mit dem Thema Lichtverschmutzung auseinander. Astronomen der Universität Wien messen $\operatorname{sie}^{7}$ und Hobby-Sterngucker tauschen sich über möglichst dunkle Orte aus. Private Umweltschützer und -schutzvereine informieren über das Thema und die Umweltämter bearbeiten es auf Bundes- und Länderebene.

\section{Vorschlag weiteres Vorgehen}

In verschiedenen Städten wird bereits die Straßenbeleuchtung nachts stark reduziert, was der Bevölkerung oft gar nicht auffällt (Posch 2013). Anderorts wird die Weniger-Beleuchtung gestaffelt und je nach Platz und Straßenzug unterschiedlich vollzogen (Isépy 2013). In manchen Fällen und je mehr Infrastruktur dafür bereits zur Verfügung steht, geht das Lichtmanagement bereits in Richtung Smart City und schaltet das Licht beispielsweise morgens individuell aus, wenn die Umgebungshelligkeit hoch genug ist. Auch wenn BürgerInnen eine Anpassung der Helligkeits-Niveaus nicht weiter auffällt, sollten Maßnahmen zur Beleuchtungsreduktion klar kommuniziert werden, denn nächtliche Beleuchtung trägt zum Sicherheitsempfinden bei.

Ein Blick nach Sloweniens zeigt, dass es möglich ist Grenzwerte zu setzen. Hier ist etwa geregelt wieviel Energie Gemeinden pro BewohnerIn und Jahr für Beleuchtung verbrauchen dürfen und wie hell Licht am Schlafzimmerfenstern sein darf. Auch darf nur in Ausnahmefällen von unten nach oben gestrahlt werden und Schautafeln müssen ab einer bestimmten Größe nach 24 Uhr ausgeschaltet werden. ${ }^{8}$

Diese Bestimmungen können als Anhaltspunkt dienen, doch sollte die aktuelle Lage in Österreich erfasst werden. Generell stellt sich die Frage, welche Beleuchtungsintensitäten und -intervalle in welchen Kontexten notwendig sind. Die Möglichkeiten bezüglich Richtbarkeit und Steuerung der LED-Technologie könnten besser genutzt werden. LED sind das energieeffizienteste Leuchtmittel, und den teilweisen ökologischen Schwierigkeiten kann mit Blaufiltern und der Nutzung warmweißer LED Abhilfe geschaffen werden.

\footnotetext{
4 Sternenweg Großmugl: orf.at/stories/3002842/.

5 International Dark Sky Association: darksky.org/our-work/conservation/idsp/parks/.

6 Naturpark Attersee-Traunsee und die Hohe Dirn in den Kalkalpen: science.orf.at/v2/stories/2931477/.

7 astro.univie.ac.at/ueber-uns/lichtverschmutzung/.

8 Zusammenfassung des deutschen Bundestages: bundestag.de/resource/blob/ 632966/7ba7c4cd1cfef87380d58376f1c2f165/WD-7-009-19-pdf-data.pdf.
} 


\section{Zitierte Literatur}

Blume, C., Hauser, T., Gruber, W.R. et al., 2020, "How does Austria sleep?" self-reported sleep habits and complaints in an online survey. Sleep Breath 24, 735-741 (2020), doi.org/10.1007/s11325-019-01982-5.

Eisenbeis G., Eick K., 2011, Studie zur Anziehung nachtaktiver Insekten an die Straßenbeleuchtung unter Einbeziehung von LEDs. Natur und Landschaft 86(7): 298-301.

Held, M.; Hölker, F.; Jessel, B. (Hg.), 2013, Schutz der Nacht Lichtverschmutzung, Biodiversität und Nachtlandschaft. Bundesamt für Naturschutz, BfN-Skripten 336, Bonn.

Isépy, S., 2013, Umweltfreundliche und effiziente öffentliche Beleuchtung in Augsburg. In: Held et al. (2013), S.169-172.

Posch, T., 2013, Besser beleuchten - Intensität, spektrale Zusammensetzung und Timing der Beleuchtung. In: Held et al. (2013), S.43-46.

TAB, 2020, Lichtverschmutzung - Ausmaß, gesellschaftliche und ökologische Auswirkungen sowie Handlungsansätze. Arbeitsbericht Nr. 186. Karlsruhe, Büro für Technikfolgen-Abschätzung beim Deutschen Bundestag, tab-beim-bundestag.de/de/pdf/publikationen/berichte/TABArbeitsbericht-ab186.pdf.

Rodenbeck, A., 2020, Chronobiologie, Enzyklopädie der Schlafmedizin, 1-5. Springer, doi.org/10.1007/978-3-642-54672-3_32. 


\section{Geräuschvolle Zukunft?}

\section{Zusammenfassung}

Lärmbelastung ist in Städten ein großes Umweltproblem und kann zur Belastung der Gesundheit führen. Unterschiedliche Ansätze und Technologien zielen auf die Reduktion von Lärm im öffentlichen Raum und für Individuen ab. Digitale Technologien unterstützen die Erfassung von Lärmquellen und akustische Stadtplanung trägt zur Verminderung von Stadtlärm bei. Gleichzeitig nehmen Lärmquellen, wie z. B. Verkehr, Klimaanlagen, oder Mini-Lautsprecher, zu. Das Spannungsverhältnis zwischen subjektivem Lärmempfinden und objektiver Lärmmessung, macht den Bedarf für Diskussion und Verhandlung wünschenswerter Klang-Zukünfte deutlich.

\section{Überblick zum Thema}

Geräusche und Lärm in der Stadt sind allgegenwärtig: Baustellen- und Straßenlärm, Musik aus Lautsprechern und Sirenen von Einsatzfahrzeugen. Das wurde vor allem im Lock-Down deutlich, als Lärmemissionen signifikant zurückgingen und plötzlich Vogelgezwitscher und Kirchturmläuten die städtischen Geräuschkulissen prägten (Arora et al. 2020). Lärmverschmutzung zählt zu den großen Umweltproblemen in der Stadt, da Lärm nicht nur Tiere beeinträchtigt (z. B. Fledermäuse, die auf Echoortung angewiesen sind, Vögel, die ihre Gesangslautstärke an den Umgebungslärmpegel anpassen ${ }^{1}$ ), sondern auch für Menschen eine gesundheitliche Belastung darstellen kann. Folgen können von hormonellen Störungen, über Depressionen bis hin zu Herz-Kreislauf-Erkrankungen reichen. ${ }^{2}$ Töne und Geräusche können aber auch positiv assoziiert sein, wenn sie z. B. den einzigartigen Klangcharakter einer Stadt hervorheben oder in Form von Musik zur individuellen Entspannung beitragen. Denkt man an Geräuschemissionen im nachbarschaftlichen Bereich, wird schnell klar, dass die Unterscheidung zwischen unerwünschtem und belastendem Schall und erwünschter, unterhaltender Beschallung sehr subjektiven Kriterien unterliegt. So kann die Party der Einen schnell zur akustischen Hölle der Anderen werden, aber auch umgekehrt, das virtuose Geigenspiel der Einen im Wohlgefallen aller resultieren. Während die medizinische Forschung Gehörschäden durch schädigende Lärmeinflüsse (das sind Lärmpegel über 80-85 dB(A)) nachweisen kann, gibt es kaum epidemiologische Studien zum Zusammenhang zwischen konkreter Lärmbelastung und Gesundheitsschädigung (Mazur et al. 2007). Es wird deutlich, dass nicht nur die eigentliche Lärmquelle, sondern viele andere Faktoren, wie bspw. der Zeitpunkt, die Dauer oder der Grund des Lärms, das individuelle Lärmempfinden beeinflussen.
Hohe Lärmbelastung in der Stadt

\section{Subjektivität des} Lärmempfindens

Viele Einflussfaktoren auf Lärmwahrnehmung

\footnotetext{
1 bbc.com/news/science-environment-54285627.

2 derstandard.at/story/2000120573929/wie-soll-die-stadt-der-zukunft-klingen.
} 
Lärmpflicht?

Beispiel E-Auto

Geräuschreduzierende Kopfhörer

Mini-Lautsprecher

Klanginstallationen

\section{Lärmminderungstechnologien und Schallschutz}

Es gibt bereits vielfältige Ansätze den Lärm im öffentlichen Raum zu mindern. Angefangen vom Rasengleis ${ }^{3}$, das den Schienenverkehrslärm bei Straßenbahnen reduzieren kann, über Bewuchsstreifen entlang von Verkehrswegen ${ }^{4}$ bis hin zu Lärmschutzwänden an Autobahnen. ${ }^{5}$ Auch die Einführung von E-Autos führt aufgrund der fehlenden Motorengeräusche in einem ersten Schritt zu einer Reduktion des Verkehrslärms. Das Beispiel E-Auto zeigt, dass Lärmminderung kein allgemeingültiges und von allen erwünschtes Ziel sein muss: Blinde und sehbehinderte Menschen nutzen $u$. a. das Motorengeräusch zur Ortung eines Fahrzeugs und verweisen auf das erhöhte Gefährdungspotenzial durch lautlose Autos. Als Konsequenz führte die EU per Verordnung ${ }^{6}$ eine „Lärmpflicht“ für Kraftfahrzeuge ein, die seit 2019 in Österreich gilt.

Neben Ansätzen, die auf die Verringerung des Lärms im öffentlichen Raum abzielen, gibt es auch Versuche zur individuellen Lärmminderung, die sogenannten geräuschreduzierenden Kopfhörer oder auf Englisch „active noise-cancellation (ANC)“" Technologie. Ursprünglich wurden sie für Flugzeugpiloten entwickelt, um besser kommunizieren zu können und ihnen Ruhe auf Langstreckenflügen zu garantieren ${ }^{7}$, heute sind sie in vielfacher Ausführung kommerziell verfügbar. Diese speziellen Kopfhörer erfassen anhand von integrierten Mikrophonen niederfrequente Geräusche aus der Umgebung und neutralisieren sie, bevor sie zum Ohr gelangen. Bei höheren Frequenzen kommen die Dämmstoffe im Kopfhörergehäuse zum Einsatz und dämpfen den Schall passiv (,noise isolation“).

\section{Geräuscherzeugende Technologien}

Auf der einen Seite zielen individuelle und öffentliche Ansätze auf die Reduktion von Lärm ab; auf der anderen Seite gibt es immer mehr Technologien, die Geräusche erzeugen. Die zunehmende Miniaturisierung von Lautsprechern und ihre technologische Weiterentwicklung (Bluetooth-Verbindung zum Smart Phone, Aufladen über tragbare Solarpanele) ermöglicht es sie überall hin mitzunehmen und auch outdoor, z. B. im Park oder Schwimmbad, zu verwenden. Die durch die unfreiwillige Beschallung entstehenden Konflikte werden oft im Kulanzweg geregelt und setzen auf die Einsicht der NutzerInnen von Lautsprechern. ${ }^{8}$ Künstlerische Interventio-

3 tramway.at/fachartikel/2013-06-04-RS-Rasengleise.pdf.

4 ait.ac.at/themen/akustik-und-laermschutz-im-bereich-verkehr/projects/laub/.

5 ait.ac.at/themen/akustik-und-laermschutz-im-bereich-verkehr/akustischeeigenschaften-und-optimierung-von-laermschutzwaenden/.

6 eur-lex.europa.eu/legal-content/DE/TXT/?uri=CELEX:32014R0540.

7 theguardian.com/technology/2017/mar/16/noise-cancelling-headphones-soundmodern-life.

8 t-online.de/digital/id_86257026/laermbelaestigung-durch-bluetooth-lautsprecherstaedte-haben-kein-rezept.html; in Österreich gibt es keine gesetzlich festgelegte Ruhezeit, jedoch kann eine Lärmstörung eine strafbare Verwaltungsübertretung darstellen. 
nen, wie z. B. Klanginstallationen im öffentlichen Raum ${ }^{9}$ oder Performances mit akustischen Elementen ${ }^{10}$, tragen ebenso zur öffentlichen Beschallung mit ausgewählter Musik bei, wobei diese zuweilen auf Bewusstseinsbildung und Auseinandersetzung mit akustischen Perspektiven abstellen.

\section{Relevanz des Themas für das Parlament und für Österreich}

Wie wird die Stadt der Zukunft klingen und welche Geräuschkulissen werden sie prägen? Die beschriebenen, heterogenen Dynamiken im Bereich Lärm verweisen auf sehr diverse Zukunftsszenarien. Vorstellbar ist, dass wir zukünftig von neuen, synthetischen Klangwelten umgeben sein werden, aber auch, dass bewusst eingerichtete Ruhezonen auf den Erhalt natürlicher Geräuschkulissen abstellen. Auch die individuelle akustische Filterblase, die durch das Tragen von Kopfhörern ermöglicht wird, könnte unsere Klangzukunft bestimmen. Die steigenden Bevölkerungszahlen und die zunehmende Anzahl geräuschemittierender Quellen (z. B. laute Klimaanlagen in Wohnhausinnenhöfen) in Städten verstärken den Trend der Lärmbelastung. Es gibt Stimmen, die Stille als zunehmenden Luxus beschreiben, den sich zukünftig möglicherweise nur mehr reiche Menschen leisten können (Thompson 2017). Doch wie kann zukünftig eine faire Nutzung des öffentlichen Guts "Stille" garantiert werden?

Der Umgang mit Geräuschen wird in vielen Disziplinen und auch transund interdisziplinär beforscht (Physik ${ }^{11}$, Medizin ${ }^{12}$, Kunst, Raumplanung, u.v.m.). Digitale Technologien erweitern den Forschungsraum auf partizipative Projekte, in denen bspw. Lärmquellen von Bewohnerlnnen anhand einer Smart Phone App identifiziert, aufgenommen und beschrieben werden können. Diese Daten werden für das Trainieren eines Algorithmus, der Lärmquellen in Sensordaten erkennen soll, verwendet. ${ }^{13}$ Darüber hinaus gibt es eine Reihe bestehender Instrumentarien, mit denen auf Lärmbelastung reagiert werden kann. Die Klangraumplanung bzw. akustische Stadtgestaltung zielt bspw. auf die Berücksichtigung von Klang in der Stadtplanung ab und versucht anhand von planerischen Finessen (z. B. begrünte Fassaden und Geleiskörper, die den Schall schlucken, verkehrsberuhigte Straßen, Bauweisen, die Straßenlärm abschirmen) den Stadtlärm zu mindern. Bei Neubauprojekten bietet die Umweltverträglichkeitsprüfung gewisse Möglichkeiten für die Abschätzung von zukünftiger Lärmbelastung.

\author{
Szenarien für die \\ Klangwelt der Zukunft?
}

Ansätze zum Umgang mit Lärm

\footnotetext{
9 Z. B. die bekannte Klanginstallation im Wunderburggraben der Ruine Dürnstein gedaechtnisdeslandes.at/kunst/action/show/controller/Kunst/werk/duernsteinklanginstallation-im-wunderburggraben-der-ruine-duernstein.html

10 koer.or.at/projekte/city-of-noise/.

11 sounds-of-matter.univie.ac.at/.

12 meduniwien.ac.at/web/en/about-us/news/detailsite/2019/news-im-november2019/sounds-and-science-music-immunology-and-life-sciences-in-graz-on-22november/.

13 zooniverse.org/projects/anaelisa24/sounds-of-new-york-city-sonyc.
} 
Hoher gesellschaftlicher Verhandlungsbedarf

\section{Vorschlag weiteres Vorgehen}

Trotz aller Bemühungen, die nach einem nachhaltigen und fairen Umgang mit Geräuschen und Lärm trachten, bleibt die Bewertung von Lärm eine große Herausforderung, die durch das Spannungsverhältnis zwischen subjektivem Lärmempfinden und objektiver Lärmmessung geprägt wird. Oft ist die Bewertung von Lärmbelastung nur anlassbezogen möglich, wobei die dabei notwendigen gesellschaftlichen Aushandlungsprozesse mitunter asymmetrischen Machtverhältnissen unterliegen, aufgrund derer nicht alle Interessen gleichermaßen Berücksichtigung finden. Daher wäre es wichtig, vermehrt Bewusstsein für die zugrundeliegenden Verhältnisse zu schaffen und Verhandlungsräume für Geräuschfragen zu etablieren, in denen kreativ und partizipativ mögliche und wünschenswerte Klang-Zukünfte diskutiert und verhandelt werden können.

\section{Zitierte Literatur}

Arora, S., Bhaukhandi, K. D. und Mishra, P. K., 2020, Coronavirus lockdown helped the environment to bounce back, The Science of the total environment 742, 140573-140573, pubmed.ncbi.nlm.nih.gov/32619844.

Mazur, H., Theine, W., Lauenstein, D., Schuster, S. und Weisner, C., 2007, Lärmrelevanz und EU-Anforderungen. Erfordernisse, Abgrenzungsund Anpassungsprozesse zum Lärmschutz im Experimentellen Wohnungs- und Städtebau (ExWoSt) des Bundesministeriums für Verkehr, Bau und Stadtentwicklung, Hannover: Bundesamt für Bauwesen und Raumordnung (BBR).

Thompson, M., 2017, Beyond Unwanted Sound: Noise, Affect and Aesthetic Moralism: Bloomsbury Publishing, books.google.at/books?id=0Vu6DQAAQBAJ. 


\section{Fernerkundung mit KI}

Erdobservationssatelliten und zunehmend auch Forschungsdrohnen sammeln große Mengen von Umweltdaten. Allein die Europäische Raumfahrtagentur (ESA) produziert beispielsweise mit den Sentinel Satelliten täglich Datenmengen in der Größenordnung von mehreren Millionen hochauflösenden Fotos. Diese stehen Open Access zur Verfügung und werden für verschiedenste Zwecke ausgewertet. Im Umweltbereich beispielsweise zur Wettervorhersage, Klimaforschung, zur Überwachung von Wasserressourcen, zur Messung von Meeres- und Luftverschmutzung oder auch für das Notfallmanagement bei Waldbränden und Erdrutschen sowie in der Präzisionslandwirtschaft.

Maschinelles Lernen wird immer häufiger zur automatischen Auswertung der Fülle von Daten aus der Fernerkundung eingesetzt, um komplexe Umweltsysteme besser zu verstehen und zu managen (Yuan et al. 2020). $\mathrm{KI}$-Einsatz kann z. B. Wettervorhersagen und Klimamodellierungen verbessern, um damit beispielsweise bessere Vorhersagen für Überflutungen oder Dürren zu treffen. KI findet auch in der Forstwirtschaft Anwendung (Sandino et al. 2018; Razavi-Termeh et al. 2020), z.B. bei der drohnenbasierten Überwachung von Schädlingsbefall oder bei Ernte, Logistik oder illegalem Holzeinschlag, oder der Vorhersage von Waldbränden.

Neben vielen Vorteilen, bringen KI-Anwendungen aber auch technische sowie ethische und rechtliche Herausforderungen mit sich. Technisch wird ein weit verbreiteter Einsatz von KI durch die Datenzugänglichkeit und den Aufbau und die Validierung der Trainingsdaten limitiert. Außerdem bringt das Algorithmendesign Unsicherheiten durch Fehler und Bias mit sich, was zu rechtlichen und ethischen Problemen führen kann [siehe Transparente Algorithmen; KI-Risiken]. Das Konzept der erklärbaren KI (explainable Al) versucht diese Risiken abzufedern (Barredo Arrieta et al. 2020). Bei komplexen Algorithmen in der Umweltfernerkundung stellt sich, nicht nur aber insbesondere im öffentlichen Sektor die Frage der Nachvollziehbarkeit und Transparenz von getroffenen Entscheidungen. Auch Verantwortungs- und Haftungsfragen sind bislang ungeklärt (POST 2020).

Die europäische Datenschutzgrundverordnung bezieht sich zwar auf die Verwendung personenbezogener Daten, allerdings sehr allgemein. Es muss rechtliche eindeutig geklärt werden, wie mit den hochauflösenden Daten der Fernerkundung umzugehen ist. Die zwei Trends von zunehmender Verfügbarkeit von Satellitenbildern mit sehr hoher räumlicher und zeitlicher Auflösung und die Auslagerung der rechenintensiven Bildanalyse haben in Verbindung mit den in naher Zukunft absehbaren Verbesserungen der Gesichtserkennungstechnologie und anderer Bilderkennungssoftware das Potenzial für die Identifizierung von Personen und bergen damit Auswirkungen auf Privatsphäre, Datenschutz und ethische Risiken (Santos/Rapp 2019). Im Vordergrund stehen auch ungeklärte Verantwortungs-, Haftungs- und Abhängigkeitsfragen: Was wenn $\mathrm{KI}$ einen falschen 
Alarm auslöst oder einen Notfall nicht erkennt? Welche Kontrollpunkte haben automatisierte Entscheidungsprozesse? Was wenn Entscheidungen auf real-time-Daten basierten Prognosen beruhen und die Daten nicht ausreichend oder fehlerhaft sind?

\section{Zitierte Literatur}

Barredo Arrieta, A., et al., 2020, Explainable Artificial Intelligence (XAI): Concepts, taxonomies, opportunities and challenges toward responsible Al, Information Fusion 58, 82-115, sciencedirect.com/science/article/pii/S1566253519308103.

POST, 2020, Remote sensing and machine learning, London: UK Parliament Parliamentary Office of Science and Technology, post.parliament.uk/research-briefings/post-pn-0628/.

Razavi-Termeh, S. V., Sadeghi-Niaraki, A. und Choi, S.-M., 2020, Ubiquitous GIS-Based Forest Fire Susceptibility Mapping Using Artificial Intelligence Methods, Remote Sensing 12(10), 1689.

Sandino, J., Pegg, G., Gonzalez, F. und Smith, G., 2018, Aerial mapping of forests affected by pathogens using UAVs, hyperspectral sensors, and artificial intelligence, Sensors 18(4), 944.

Santos, C. und Rapp, L., 2019, Satellite Imagery, Very High-Resolution and Processing-Intensive Image Analysis: Potential Risks Under the GDPR, Air and Space Law.

Yuan, Q., et al., 2020, Deep learning in environmental remote sensing: Achievements and challenges, Remote Sensing of Environment 241, 111716, sciencedirect.com/science/article/pii/S0034425720300857. 


\section{Mikroplastik - Abrieb der Zivilisation}

\section{Zusammenfassung}

Mikroplastikverschmutzung ist weit mehr als ein Meeresverschmutzungsproblem, nachdem Mikroplastik weltweit auch in Ackerböden, Städten, Flüssen, der Atmosphäre, im Eis der Antarktis und im menschlichen Darm gefunden wird. Mikroplastik wird entweder in industriellen Prozessen hergestellt, etwa in Form von Pellets zur Weiterverarbeitung, oder entsteht durch mechanische Zerkleinerung und Verwitterung von Plastikprodukten, z.B. Reifenabrieb. Die meisten Plastikarten sind so gut wie nicht oder nur sehr langsam biologisch abbaubar, damit akkumuliert sich bei weltweit zunehmender Plastikproduktion auch die Mikroplastikbelastung der Umwelt. Plastik enthält Weichmacher, die hormonell wirksam sind. Krankheitserreger sowie krebserregende Schadstoffe lagern sich konzentriert an Mikroplastikpartikeln ab. Sie werden von Tieren gefressen und sogar von Pflanzen aufgenommen und gelangen so in die menschliche Nahrungskette.

\section{Überblick zum Thema}

Meeresverschmutzung durch Mikroplastik ist seit Ende der 1960er-Jahre bekannt. Frühe wissenschaftlichen Studien zeigten die Verbreitung von sich immer weiter zerkleinernden Mikroplastikpartikeln in Meeren und dass sich an deren Oberfläche Schadstoffe wie PCB in hoher Konzentration ablagern, die dann von Fischen oder Meeresvögeln gefressen werden und so auch in die menschliche Nahrungskette gelangen. Primäres Mikroplastik wird in industriellen Prozessen hergestellt oder entsteht während der Nutzung von Plastikprodukten, während sekundäres Mikroplastik durch mechanische Zerkleinerung und Verwitterung von (Makro-)Plastikprodukten nach Ende ihrer Nutzungsphase entsteht. Mikroplastikgranulat wird in Form kleine Pellets bis zur Größe von wenigen Millimetern, als Rohstoff zur Fertigung von Plastikprodukten hergestellt. Dieses Rohmaterial wird oft unbeabsichtigt bei der Herstellung als auch beim Transport verschüttet und so an die Umwelt abgegeben und wird deshalb häufig in der Nähe von Kunststoffproduktionsstätten und Laderampen in erhöhter Konzentration gefunden (Karlsson et al. 2018). Mikroplastikgranulat wird auch in kosmetischen Produkten wie Duschgels, Peelings oder Zahnpasta eingesetzt. Verglichen mit anderen Quellen sind Mikroplastikemissionen aus Kosmetika eine relativ niedrige Belastung, da die Kügelchen aber besonders klein und leicht sind, können sie relativ einfach Kläranlagen passieren und damit in Flüsse und Ozeane gelangen (Ziajahromi et al. 2017).
Mikroplastik in Meeren seit der 60er-Jahren bekannt

Unterscheidung zweier Arten von Mikroplastik

primäres Mikroplastik aus industrieller Herstellung als Rohstoff und in Kosmetika [Typ A] 
primäres Mikroplastik durch Abrieb während der Nutzung [Typ B]: vor allem Transportsektor

Mikrofasern in Wasser und Luft

sekundäres

Mikroplastik:

Fragmentierung nach

Ende der

Nutzungsphase

Möglichkeit,

Verantwortlichkeiten zu klären

Mikroplastik weltweit in allen Ökosystemen verbreitet

auch Ackerböden belastet
Primäres Mikroplastik vom Typ B macht den Großteil des emittierten primären Mikroplastiks aus (ca. 90 \%). Es entsteht während der Nutzung verschiedener Kunststoffprodukte. Reifenabrieb wird hier als die größte Quelle eingeschätzt, gemeinsam mit Asphalt und Straßenmarkierungsabrieb ist damit der Transportsektor für mehr als $50 \%$ der primären Emissionen verantwortlich (Bertling et al. 2018). Andere Quellen sind Baustellen, Landwirtschaft, Sportplätze, Schuhsohlen und Plastikverpackungen. Mikrofasern aus synthetischer Kleidung, die beim Waschen freigesetzt werden, sind eine weitere wichtige Quelle. Sie machen zwar nur geschätzt $3 \%$ der primären Mikroplastikemissionen aus (Bertling et al. 2018), sind gleichzeitig aber ein großer Teil (35\%) des Mikroplastiks, das in marinen Ökosystemen gefunden wird (Henry et al. 2019). Diese hohen Konzentrationen von Fasern sind wahrscheinlich darauf zurückzuführen, dass sie aufgrund ihrer physikalischen Eigenschaften nur sehr schlecht von Kläranlagen zurückgehalten werden. Auch die in der Luft befindlichen Fasern werden wahrscheinlich von synthetischer Kleidung und weichen Möbeln und Teppichen freigesetzt (Abbasi et al. 2019). Sekundäres Mikroplastik entsteht vor allem durch Verwitterung und Fragmentierung von makroplastischen Produkten in der Umwelt (Bertling et al. 2018). Verwitterung wird durch UV-Einstrahlung, Oxidation, mikrobielle Aktivität und mechanischen Abrieb, etwa durch Wellen-, Wind- oder Bodenbewegungen hervorgerufen (Liebmann 2015). Am effektivsten ist die Zerkleinerung aber an Stränden durch Brandung, Sandbewegungen und UV-Licht. An Land wird weggeworfener Plastikmüll zu einer Quelle von Mikroplastik, die Datenlage hierzu ist aber schwach. Diese Klassifizierung in primäres Mikroplastik Typ A und B sowie sekundäres Mikroplastik ist sinnvoll, um die Emissionen mit Quellen und Verantwortlichkeiten zu verbinden. Die Quellen von primärem Mikroplastik gehören noch jemandem, während die Quellen von sekundärem Mikroplastik in der Regel niemandem mehr zuzuordnen sind.

Mikroplastik ist weltweit in den meisten Bereichen der Umwelt zu finden, es ist einer der am weitesten verbreiteten Schadstoffe im Meer (Chen et al. 2019) und sogar in Krebsen in über $10.000 \mathrm{~m}$ Meerestiefe nachgewiesen. Nachdem sich Mikroplastik in allen Meeren und an Stränden auf der ganzen Welt nachweisen lässt, kamen in den letzten Jahren Nachweise für Vorkommen in Flüssen und Seen dazu. Auch im Trinkwasser ist Mikroplastik omnipräsent (Danopoulos et al. 2020). Mikroplastik wurde ebenso in erheblichen Mengen in der Atmosphäre gefunden, in der Antarktis (Waller et al. 2017), im Straßenstaub sowie im Darm von Tieren und Menschen. Über Transportprozesse und -wege gibt es derzeit trotzdem noch relativ wenig gesicherte Daten, abgesehen davon, dass Wind- und Wassertransport eine wichtige Rolle spielen.

Aufmerksamkeit erhielt in letzter Zeit vor allem die Verbreitung in Ackerböden. Abrieb von in der Landwirtschaft eingesetzten Plastikplanen, in Plastik eingekapselter Kunstdünger und weggeworfener Müll sind Quellen für Mikroplastik. Weiters ist aber auch die Ausbringung von Klärschlamm auf landwirtschaftlichen Flächen mitverantwortlich. Ein Teil der leichten Kunststoffpartikel schwimmt in Kläranlagen auf, wird gemeinsam mit Fett 
in den Faulturm gepumpt und kommt damit in den Klärschlamm. Mikroplastik kann je nach Konzentration Bodenparameter wie Feuchtigkeit, Belüftung sowie mikrobielle Aktivität und Zusammensetzung erheblich negativ beeinflussen.

In der Umwelt widerstehen die meisten Plastikarten dem Abbau durch Mikroorganismen, da diese die Polymere nicht verstoffwechseln können. Auch wenn Plastik physikalisch immer weiter zerkleinert wird, verschwindet es nicht und reichert sich immer mehr in der Umwelt an, zumindest im menschlichen Zeithorizont. Zwar werden auch vermehrt biologisch abbaubare Plastikarten entwickelt, ihr Anteil an der Gesamtplastikproduktion ist aber gering. Auch ist sogenanntes Bioplastik nicht mit „biologisch abbaubar" gleichzusetzen. Bei dieser Art von Plastik geht es vor allem darum, dass als Ausgangsstoff nicht Erdöl, sondern ein nachwachsender Rohstoff wie Mais verwendet wird, die entstehenden Polymerketten sind damit aber nicht zwangsläufig schnell biologisch abbaubar. Das wird bei Produkten wie Lebensmittelbehältern auch nicht gewünscht. Eine neue Studie ein Bakterium, das Polyethylen (PET) zum Energie- und Kohlenstoffgewinn zu in der Natur vorkommenden Monomeren zu spalten kann. Eine großflächige Anwendung auf das bestehende gravierende Mikroplastikproblem ist aber aus einer Vielzahl von technischen Gründen und nicht abschätzbaren Risiken und Folgen nicht absehbar.

Mikroplastik kann mehrere schädliche Auswirkungen auf Menschen und andere Organismen, Populationen und ganze Ökosysteme haben, die mitunter sehr schwer abschätzbar sind (Bertling et al. 2018). Derzeit sind die Wissenslücken über mögliche Schäden enorm, da es in den verschieden Bereichen der Umwelt sehr viel verschiedene Einflussfaktoren gibt (Liebmann 2015). Gesicherte Aussagen lassen sich bezogen auf Meeresorganismen machen, weil diese am längsten und besten erforscht sind. Sobald Mikroplastik aufgenommen wird, kann es entweder aus dem Organismus ausgeschieden werden, wodurch keine dauerhafte Wirkung entsteht. Oder es verbleibt im Organismus, was zu lokalen Entzündungen führen, den toxikologischen Stress erhöhen, das Wachstum beeinträchtigen, Lebertätigkeit einschränken oder die Nahrungsaktivität verringern kann (Auta et al. 2017). Außerdem lagern sich persistente organische Schadstoffe, Schwermetalle und andere Umweltschadstoffe auf der wasserabweisenden Oberfläche des Mikroplastiks ab, einige von ihnen sind hoch krebserregend.

Eine Vielzahl umweltbeständiger Chemikalien wie Pestizide oder PCB, die sich an Mikroplastikpartikeln ablagern, wirken im Hormonsystem wie das weibliche Sexualhormon Östrogen und können damit erhebliche gesundheitliche Auswirkungen haben. Das gilt auch für einige dem Plastik absichtlich zugesetzter Additive wie Weichmacher (Phtalathe). Im Meer nehmen vor allem filtrierende Organismen wie Muscheln oder Plankton Mikroplastik auf. In Böden sind es beispielsweise Regenwürmer und sogar Pflanzen. Neben den schädlichen Folgen für diese Organismen akkumuliert sich Plastik im Nahrungsnetz, wenn sie gefressen werden. Auf diesem Weg gelangen Plastik und die Schadstoffe, die es transportiert, auch auf den menschlichen Teller.
Nicht-Abbaubarkeit vieler Plastikarten und steigende Produktion

Plastikabbau durch

Bakterien nicht absehbar

schädliche

Auswirkungen

Organismen,

Populationen und

Ökosysteme

Ausscheidung oder Gewebeentzündungen

Oberfläche zieht

Schadstoffe an

hormonell wirksame Weichmacher

Akkumulation im Nahrungsnetz 


\author{
Datenlage für Österreich \\ schlecht
}

\section{Relevanz des Themas für das Parlament und für Österreich}

Die Datenlage für Mikroplastikemmissionen und Belastungen in Österreich ist sehr schlecht, mit wenigen Studien. Wahrscheinlich kann von ähnlichen Größenordnungen wie in anderen europäischen Ländern ausgegangen werden. In Deutschland werden die Mikroplastikemmissionen auf insgesamt 330.000 Tonnen pro Jahr oder $4 \mathrm{~kg}$ pro Kopf geschätzt (Bertling et al. 2018). Zwar gibt es erste Regulierungen wie das europaweite Verbot von Einweggegenständen aus Plastik (EP 2019), diese greift aber zu kurz, verbietet sie doch nur die zehn am häufigsten im Meer gefundenen Gegenstände, wie Wattestäbchen, Strohhalme oder Einweggeschirr. Da eine Reinigung der Umwelt von entstandenem Mikroplastik derzeit eigentlich nicht möglich ist, müssen andere Wege wissenschaftlich und politisch evaluiert werden. Ein Weg wäre die massive Reduktion der Plastikproduktion gepaart mit einer Überführung in eine vollständige Kreislaufwirtschaft. Aber auch hier bleiben die Emissionen großer Quellen wie der Reifenabrieb oder verlorenen Fischernetze bestehen. Hier könnten umweltfreundlichere Materialien gefördert werden. Voraussetzung einer besseren Risikobewertung ist auch die Einführung gemeinsamer Beprobungsstandards, zumindest in der EU (UBA 2020).

\section{Vorschlag weiteres Vorgehen}

Es wird vorgeschlagen, zu diesem auch für Österreich höchst relevanten Thema - Böden, Luft und Gewässer sind betroffen - eine umfassende Foresight \& TA-Studie unter Einbeziehung aller einschlägigen Fachbereiche, insbesondere der Umwelttoxikologie, durchzuführen, um eine fundierte Basis für eine vertiefte Auseinandersetzung mit einer Vielzahl möglicher Maßnahmen zur Reduktion der Emissionen zu ermöglichen.

\section{Zitierte Literatur}

Abbasi, S., Keshavarzi, B., Moore, F., Turner, A., Kelly, F. J., Dominguez, A. O. und Jaafarzadeh, N., 2019, Distribution and potential health impacts of microplastics and microrubbers in air and street dusts from Asaluyeh County, Iran, Environ Pollut 244, 153-164, ncbi.nlm.nih.gov/pubmed/30326387.

Auta, H. S., Emenike, C. U. und Fauziah, S. H., 2017, Distribution and importance of microplastics in the marine environment: A review of the sources, fate, effects, and potential solutions, Environ Int 102, 165-176, ncbi.nlm.nih.gov/pubmed/28284818.

Bertling, J., Bertling, R. und Hamann, L., 2018, Kunststoffe in der Umwelt: Mikro- und Makroplastik, Fraunhofer Umsicht.

Chen, Q. Q., Zhang, H. B., Allgeier, A., Zhou, Q., Ouellet, J. D., Crawford, S. E., Luo, Y. M., Yang, Y., Shi, H. H. und Hollert, H., 2019, Marine microplastics bound dioxin-like chemicals: Model explanation and risk assessment, Journal of Hazardous Materials 364, 82-90.

Danopoulos E, Twiddy M, Rotchell JM (2020) Microplastic contamination of drinking water: A systematic review. PLOS ONE 15(7): e0236838. doi.org/10.1371/journal.pone.0236838. 
EP, 2019, Proposal for a Directive of the European Parliament and of the Council on the reduction of the impact of certain plastic products on the environment, European Parliament, eur-lex.europa.eu/legalcontent/EN/HIS/?uri=COM\%3A2018\%3A340\%3AFIN.

Henry, B., Laitala, K. und Klepp, I. G., 2019, Microfibres from apparel and home textiles: Prospects for including microplastics in environmental sustainability assessment, Science of the Total Environment 652, 483-494.

Liebmann Bettina, Sexlinger Katharina (2020) Mikroplastik in der Umwelt. Statusbericht 2019. Wien, 2020, Reports, Band 0727, Hg. Umweltbundesamt, ISBN: 978-3-99004-547-348 S.

Karlsson, T. M., Arneborg, L., Brostrom, G., Almroth, B. C., Gipperth, L. und Hassellov, M., 2018, The unaccountability case of plastic pellet pollution, Mar Pollut Bull 129(1), 52-60, ncbi.nlm.nih.gov/pubmed/29680567.

Liebmann, B., 2015, Mikroplastik in der Umwelt. Vorkommen, Nachweis und Handlungsbedarf, BMLFUW.

Waller, C. L., Griffiths, H. J., Waluda, C. M., Thorpe, S. E., Loaiza, I., Moreno, B., Pacherres, C. O. und Hughes, K. A., 2017, Microplastics in the Antarctic marine system: An emerging area of research, Sci Total Environ 598, 220-227, ncbi.nlm.nih.gov/pubmed/28441600.

Ziajahromi, S., Neale, P. A., Rintoul, L. und Leusch, F. D., 2017, Wastewater treatment plants as a pathway for microplastics: Development of a new approach to sample wastewater-based microplastics, Water Res 112, 93-99, ncbi.nlm.nih.gov/pubmed/28160700. 


\section{Vertrauenswürdige Blockchains}

\section{Zusammenfassung}

Eine Blockchain ist eine dezentral organisierte Datenbank, die es ermöglicht, Transaktionen zwischen Akteuren - Privatpersonen, Unternehmen und öffentlichen Einrichtungen - dezentral zu dokumentieren, digital abzubilden und zu authentifizieren. Die Technologie gewährleistet, dass die Interaktionen genauso wie dokumentiert stattgefunden haben und stellt sicher, dass die Dokumentation nicht verändert werden kann. Neben den bekanntesten Anwendungen, den Kryptowährungen wie Bitcoin, werden Blockchains (auch: distributed ledger technology) zurzeit für weitere Anwendungen in Wirtschaft und Staat entwickelt: Für Eigentumsurkunden, Verträge, Versicherungen, Lizenzen etc. Blockchains haben das Potential, monopolistische Geschäftsmodelle wie Airbnb, ebay und Uber zu überwinden und AnbieterInnen und KundInnen direkt zu verbinden. Allerdings: Wenn Blockchain als Technologie allgegenwärtig werden würde, so würde damit der Lebensalltag aller BürgerInnen umfassend abgebildet und für andere im Zeitverlauf eindeutig nachvollziehbar sein. Eine personen-basierte Blockchain würde bedeuten, dass alle Handlungen im biographischen Verlauf gespeichert werden. Das Missbrauch-Potential ist damit enorm, aber bisher nicht thematisiert.

\section{Überblick zum Thema}

Die bekannteste Anwendung eines Blockchain-Algorithmus ist die Kryptowährung Bitcoin im Finanzbereich (siehe Thema „Fintechs - Revolution des Bankenwesens?". Eine Blockchain ist eine kryptografisch verbundene Kette von Blöcken. Diese Blöcke werden in einem bestimmten Zeitintervall erstellt, enthalten Transaktionen, die die Teilnehmerlnnen des Systems als ausgeführt akzeptieren, sodass z. B. ein Block bei Erhalt als akzeptiert gilt und damit die zugrundliegende Transaktion zu einem Bestandteil des Systems wird. Da alle Teilnehmerlnnen eine Kopie davon besitzen, und die vorangegangenen Datensätze mit den nachfolgenden gekoppelt und gespeichert sind, gelten die Abfolge und die einzelnen Transaktionen als gesichert gegen nachträgliche Manipulation. Beratungsfirmen und Überblicksarbeiten verweisen auf immer neue Blockchain-Anwendungen (Zheng et al. 2018). Anwendungen zeigen sich im Sektor von Grundbüchern und Landverwaltung (Bennett et al. 2019).

Das Versprechen lautet, dass die Technik Vertrauen automatisieren kann und damit Instanzen überflüssig macht, die Vertrauen schaffen und dadurch Kosten verursachen. Das Disruptionspotenzial der Blockchain ergibt sich aus dem Charakter einer Peer-to-Peer-Infrastruktur, die Transaktionen ohne Intermediäre ermöglicht. Da Intermediäre im Finanzbereich eine 
Smart Contracts

Anwendungen, die in Europa thematisiert werden größere Rolle als in anderen Wirtschaftsbereichen spielen, wird die Technologie in diesem Bereich am stärksten diskutiert. ${ }^{1}$

Die Funktionalität von Blockchains lässt sich erweitern. Eine der vieldiskutierten Erweiterungen sind Smart Contracts, die beim Zusammentreffen von bestimmten Bedingungen automatisch ausgeführt werden. Da diese Smart Contracts beliebig kompliziert sein können, die Komplexität von etablierten Verträgen damit abbilden können, gelten sie als Mittel, klassische Verträge zu ersetzen. Der Ersatz besteht in der Plattform und der Automatisierung, da die Verträge eine neue Form (digital) und einen neue Funktionsweise erhalten. Doch die Sicherheit des Systems steht zur Diskussion: Der Slogan der Entwicklercommunity von Blockchains heißt: Code is Law und genau diese Absolutheit, dass Smart Contracts immer exakt so ausgeführt werden, wie sie geschrieben sind, hat auch bereits zu ersten Sicherheitsbedenken geführt. Denn wenn Hackerlnnen sich einen „Fehler" im Smart Contract zunutze machen, könnten sie das System knacken - wobei die Rechtslage komplex ist, da das nicht-intendierte Handeln der HackerInnen gerade Teil des Codes ist, dem alle NutzerInnen zugestimmt haben. In der Startup-Szene, in der viele Blockchain-Anwendungen entwickelt werden, wird das Scheitern nicht als Problem gesehen, vielmehr ist das "fail fast" ein integraler Bestandteil der Innovationskultur. ${ }^{2}$ Für Anwendungen im öffentlichen Bereich ist dagegen eine Blockchain-Innovationsdynamik notwendig, die die möglichen Folgen umfassend antizipiert, um gerade vertrauenswürdige Anwendungen zu generieren.

Auf europäischer Ebene werden vielfältige Blockchain-Anwendungen thematisiert (Boucher et al. 2017): Neben Währungen ist die Technologie interessant für die Verwaltung von digitalen Inhalten, da sich ein entsprechendes Rechte-Management in Blockchain integrieren ließe. Im Bereich von Patenten könnte es möglich werden, über Blockchain Rechte zu verwalten. Im E-Voting sind Blockchain-unterstützte Systeme mit der Erwartung verknüpft, Mechanismen der direkten Demokratie zu vereinfachen. Blockchain-basierte Dienstleistungen im E-Government und bei der elektronischen Stimmabgabe sollen zu einer transparenteren, dezentralisierten Demokratie beitragen können. ${ }^{3}$ Das Europäische Parlament hat Ende 2018 die Europäische Union und ihre Mitgliedstaaten aufgefordert, bei Normung und Sicherheit der Blockchain-Technologie eine Führungsrolle zu übernehmen und gemeinsam mit internationalen Partnern und Interessenvertretern die Voraussetzungen zur Einführung der Technologie im Bereich Handel und im Lieferkettenmanagement mit zu erarbeiten. Dabei geht es insbesondere um Anwendungen der Blockchain-Technologie auf dem Gebiet des internationalen Handels. ${ }^{4}$

\footnotetext{
1 Siehe zur Parlamentsdiskussion: parlament.gv.at/PAKT/VHG/XXVI/J/J_00382/index.shtml.

2 deutschlandfunk.de/die-welt-veraendern-visionen-und-wahrheiten-ausder.740.de.html?dram:article_id=378079.

3 aeon.co/essays/how-blockchain-will-revolutionise-far-more-than-money.

4 europarl.europa.eu/doceo/document/A-8-2018-0407_DE.html.
} 
Den vielfältigen Bottom-up-Prozessen der Entwicklung und Erprobung von Blockchains steht noch kein Rahmen gegenüber, der aus einer längerfristigen Zukunftsperspektive heraus und über die verschiedenen Anwendungen hinaus Design-Prinzipien zur Verfügung stellt, die es den unterschiedlichen Akteuren ermöglichen würden, den verschiedenen Anforderungen über die eigene Anwendung hinaus gerecht zu werden ${ }^{5}$. Dies ist jedoch essentiell, um das Potenzial der Blockchain, institutionelles Vertrauen aufzubauen, auszuschöpfen.

\section{Relevanz des Themas für das Parlament und für Österreich}

In ökonomischer Hinsicht ist die zukünftige wirtschaftliche Bedeutung des Blockchain-Sektors unklar, aber auch mögliche Nutzungsbedingungen (Nutzung der Währungen, Akzeptanz und rechtlicher Rahmen; Privatsphäre).

Blockchain-Währungen: Es besteht hohe Unsicherheit im Hinblick auf die Zukunft des Bankensektors, international und in Österreich und im Hinblick auf den KonsumentInnen-Schutz im internationalen Feld.

Nutzen: Es gibt offene Fragen, wer zukünftig unter welchen Bedingungen von dieser Technologie profitieren kann und wie sie die Gesellschaft verändern kann.

Sicherheit von Blockchain-Anwendungen: Wie unangreifbar sind Blockchains, welche Hacking-Risiken sind abzusehen und wie kann mit innen umgegangen werden kann?

Ökologie \& Energie: Auf umweltpolitischer Ebene stellt sich die Frage des Energieverbrauchs beim Mining von Kryptowährungen und welche Lösungsansätze hinsichtlich Energiefragen zu verzeichnen sind.

Die Blockchain-Technologie bietet umfassende Anwendungsmöglichkeiten in Wirtschaft und öffentlicher Verwaltung und hat damit eine politikfeldübergreifende Relevanz.

Blockchains können disruptive Auswirkungen auf das Rechtssystem haben und bedürfen daher einer antizipierenden Politik. Aktuelles Beispiel sind Smart Contracts: Wenn durch ProgrammiererInnen Vereinbarungen in ausführbaren Code übersetzt werden, treffen diese Entscheidungen darüber, wie diese Verträge in der Praxis umgesetzt werden, hätten eine höhere rechtliche Verantwortlichkeit und sind zugleich nicht entsprechend ausgebildet. Die Beurteilung von Vertragsstreitigkeiten und die Durchsetzung von Vertragsklauseln werden Herausforderungen darstellen, wenn sich Blockchains wie erwartet entwickeln.

Die Kompatibilität der Blockchain-Technologie zum politischen Modell Österreichs und die Frage, welche Dienstleistungen der öffentlichen Verwaltung mit der Technologie entwickelt werden könnten, ist eine politikfeldübergreifende Frage.

5 Wie z.B. den Schutz persönlicher Daten, vgl. Zyskind, et al. (2015). 
Seit Ende 2018 werden die interdisziplinären Kompetenzen im Bereich der Grundlagen und der Anwendung von Blockchain-Technologien in Österreich in dem COMET Zentrum (K1) des Austrian Blockchain Centers $(A B C)$ gebündelt. ${ }^{6}$

\section{Vorschlag weiteres Vorgehen}

Im Rahmen einer Langstudie würde zunächst ein systematischer Überblick zu den aktuellen Anwendungsgebieten und technologischen Herausforderungen der Blockchain-Technologie erstellt werden. Dabei würden neben technischen Entwicklungen auch die bereits wissenschaftlich ausgewerteten Erfahrungen von Anwendungen in verschiedenen Ländern und in unterschiedlichen Branchen auf die Situation in Österreich bezogen werden. Weiterhin klärungsbedürftig ist zum einen, wie stark sich die Blockchain-Technologie insgesamt und in den verschiedenen Branchen durchsetzen wird, und zum anderen, was es für Auswirkungen hat, wenn die Technologie in Zukunft von wenigen zentralen Plattformen bestimmt wird oder aber selbst stark dezentralisiert implementiert wird.

In einem weiteren Schritt würden österreichische Stakeholder aus verschiedenen Branchen, aus Verwaltung, Blockchain-Anwenderlnnen und -Entwicklerlnnen etc. identifiziert werden. Ziel wäre es, einen Rahmen zu entwickeln, der das in Österreich bestehende Innovationspotential umfassend und zukunftsorientiert nutzbar macht. Die aktuelle Situation verweist auf ein bereits bestehendes Knowhow unterschiedlicher Akteure und bietet noch einen hohen Gestaltungsspielraum hinsichtlich der Innovationspfade von zukünftigen Blockchain-Anwendungen. Die Studie würde den möglichen Handlungsbedarf identifizieren und die Anforderungen definieren, die für die langfristige Nutzung der Blockchain-Technologie in der österreichischen Wirtschaft und Verwaltung zentral sind.

\section{Zentrale weiterführende Quellen:}

Bennett, R. M., Pickering, M. und Sargent, J., 2019, Transformations, transitions, or tall tales? A global review of the uptake and impact of NoSQL, blockchain, and big data analytics on the land administration sector, Land Use Policy 83, 435-448.

Boucher, P., Nascimento, S. und Kritikos, M., 2017, How blockchain technology could change our lives, im Auftrag von: Scientific Foresight Unit (STOA), European Parliament.

Zheng, Z. B., Xie, S. A., Dai, H. N., Chen, X. P. und Wang, H. M., 2018, Blockchain challenges and opportunities: a survey, International Journal of Web and Grid Services 14(4), 352-375.

Zyskind, G., Nathan, O., Pentland, A. und leee, 2015, Decentralizing Privacy: Using Blockchain to Protect Personal Data, 2015 leee Security and Privacy Workshops (Spw), 180-184. 6 wu.ac.at/universitaet/news-und-events/news/details-news/detail/weltweit-
groesstes-blockchain-kompetenzzentrum-in-wien-genehmigt-1. 


\section{Das Netz der bewegten Dinge}

Die Vernetzung der Dinge (Internet of Things oder kurz loT) findet in vielen Bereichen statt, wie zum Beispiel der vernetzte Kühlschrank, der selbständig Lebensmittel bestellt, oder der Fernzugriff mittels Smartphone auf verschiedene Geräte, wie Überwachungskameras, Beleuchtung oder die Heizung. Alle genannten Beispielen sind unbewegliche Dinge die im Internet of Things vernetzt sind.

Ein eigenes Anwendungsfeld ist das Netz der bewegten Dinge (Internet of Moving Things, loMT). Das sind Geräte/Dinge, die vernetzt und zudem in irgendeiner Form in Bewegung sind, wie zum Beispiel Roboter, (autonome) Fahrzeuge, oder auch Dinge die Menschen mit sich herumtragen, wie etwa Smartphones, smarte Kleidung oder andere Wearables. Durch die Vernetzung können sich diese Dinge/Geräte selbst (Geschwindigkeit, Bewegungsrichtung, aber auch ihre Umgebung vermessen (Temperatur, Geräusche, Luftverschmutzung etc.). Im Smart City Bereich sollen so auch die Vorteile von edge-, fog- oder Cloud-computing genutzt werden (Cao et al. 2020).

In der Robotik könnte die Vernetzung z. B. über Cloud-Dienste zum Austausch gesammelter Daten, „Lernerfahrungen“ bzw. Algorithmen genutzt werden. Interessante Anwendungsfelder für das Internet der bewegten Dinge ergeben sich vor allem im Bereich der Mobilität. Viele Neuwagen unterstützen bereits den SIM-Karten-Standard, Tendenz steigend. Das ermöglicht die Vernetzung der Fahrzeuge untereinander, aber auch zum Hersteller. Ferndiagnostik, Echtzeitnavigation und verschiedene Infotainment-Services können so zur Verfügung gestellt werden und sollen den Fahrkomfort und das Fahrerlebnis erhöhen. Weitere potentielle Vorteile ergeben sich über die Datensammlung des Mobilitätsverhaltens, welches für ein effizienteres Verkehrssystem genutzt werden könnte. Neben der Steigerung der Effizienz im Verkehrssektor, verspricht man sich durch die zunehmende Vernetzung der Fahrzeuge auch eine erhöhte Verkehrssicherheit.

Die zunehmende Vernetzung hat aber nicht nur Vorteile. Es stellt sich die Frage, wie das Grundrecht auf informationelle Selbstbestimmung geschützt werden kann, vor allem im Hinblick auf die Nutzung der Daten durch Dritte (Krieger-Lamina 2016). Die Vernetzung eröffnet zudem potentielle Einfallstore für HackerInnen, was gravierende Auswirkungen haben kann. 2015 haben zwei Hacker auf diese Gefahr hingewiesen. Sie haben es geschafft einen zwei Tonnen schweren SUV zu kapern. Es war ihnen somit möglich das Fahrzeug gänzlich zu steuern, angefangen bei der Klimaanlage hin zu Lenkbewegungen und sogar das Bremsen war möglich. ${ }^{1} 2016$ wiesen Hacker auf eine Sicherheitslücke eines Elektroautoherstellers hin. Sie konnten aus einer Entfernung von $19 \mathrm{~km}$ auf das Fahrzeug zugreifen.

1 wired.com/video/hackers-wireless-jeep-attack-stranded-me-on-a-highway. 
Nach einem Update wurde die Sicherheitslücke beseitigt. ${ }^{2}$ Das Tencent Keen Security Lab hat Anfang 2019 den "Autopiloten“ eines bekannten Elektroautoherstellers mittels kleiner weißer Aufkleber, die auf der Fahrbahn angebracht wurden, ausgetrickst. Der Autopilot des Fahrzeugs wurde so dazu gebracht auf die Gegenfahrbahn zu wechseln. ${ }^{3}$ Dieses Beispiel zeigt anschaulich, dass die eingesetzten Machine-Learning-Systeme nicht fehlerfrei sind. Ob die zunehmende Vernetzung der (bewegten) Dinge solche Situationen verhindern würde und nicht wohlmöglich neue Einfallstore eröffnen würde, bleibt eine offene Frage.

Auch auf Österreichs Straßen sind bereits Fahrzeuge unterwegs, die vernetzt sind. Die aktuellen Bestrebungen, Österreich in den kommenden Jahren zu einem Vorreiter der neuen Mobilfunkgeneration (5G) zu machen, hätten einen förderlichen Einfluss auf die zunehmende Vernetzung bewegter Dinge, zumal es ein explizites Ziel ist diese Technologie in der digitalen Verkehrsinfrastruktur einzusetzen (BMVIT 2018). Das Ziel hierbei ist es, die Effizienz und Sicherheit auf österreichischen Straßen zu erhöhen und zugleich die internationale Wettbewerbsfähigkeit der Unternehmen zu gewährleisten. Mit den genannten Beispielen kristallisieren sich neue Handlungsfelder im Bereich der IT-Sicherheit heraus. Die Relevanz ist auch seitens des Verbraucherlnnenschutzes gegeben.

\section{Zitierte Literatur}

BMVIT, 2018, 5G-Strategie Österreichs Weg zum 5G-Vorreiter in Europa, April 2018, Wien: Bundesministerium für Verkehr, Innovation und Technologie: bundeskanzleramt.gv.at/documents/131008/787823/15_11_5G_Strat_ NB.pdf/522ef619-e0d3-49cf-99a2-ec5ef678347b.

Cao, H.; Wachowicz, M. A (2020) Holistic Overview of Anticipatory Learning for the Internet of Moving Things: Research Challenges and Opportunities. ISPRS Int. J. Geo-Inf., 9, 272. mdpi.com/2220-9964/9/4/272/pdf.

Krieger-Lamina, J., 2016, Vernetzte Automobile. Datensammeln beim Fahren - von Assistenzsystemen zu autonomen Fahrzeugen. Endbericht, 2016-08-31, Wien: Institut für Technikfolgen-Abschätzung (ITA): epub.oeaw.ac.at/ita/ita-projektberichte/2016-02.pdf.

2 zdnet.de/88279165/tesla-model-s-sicherheitsforscher-hacken-elektroauto-ausder-ferne/?inf_by=5a03063e681db8cf478b467d.

3 futurezone.at/produkte/kleine-sticker-auf-der-strasse-lassen-tesla-aufgegenfahrbahn-wechseln/400453735 


\section{Robotik in der Landwirtschaft}

Eine zunehmende Technisierung und Automatisierung des Alltags sind auch im Bereich der Landwirtschaft zu verzeichnen. Bei diesen innovativen Agrartechnologien spielt neben der Zunahme an datenintensiven Anwendungen (Big Data in der Landwirtschaft, precision farming) ${ }^{1}$ Robotik eine zentrale Rolle. Roboteranwendungen wie zum Beispiel die autonome und präzise Aussaat, automatisierte Unkrautbekämpfung, Düngung und Obsternte, aber auch die Automatisierung der Milchproduktion über Melkroboter sind Technologien, die bereits in Erprobung bzw. im Einsatz sind. ${ }^{2}$ Autonome Traktoren gibt es bereits als Prototypen; diese könnten bei großen Feldern auch im sog. Platooning-Betrieb (als mehrere fahrerlose Maschinen an ein Führungsfahrzeug virtuell gekoppelt) eingesetzt werden. Für die eher als kleinräumig zu bezeichnende Landwirtschaft in Österreich sind viele der Anwendungen zum aktuellen Stand nicht rentabel und wahrscheinlich wenig sinnvoll. ${ }^{3}$ Auch hierzulande interessant könnten allerdings kleinere Roboter sein, die bereits auf bei weniger Fläche rentabel sein können und Daten für die Zucht sammeln ${ }^{4}$ oder Unkraut bekämpfen. ${ }^{5}$ Allgemein stellt sich die Frage welchen Einfluss, global gesehen diese Entwicklungen für die Landwirtschaft in Österreich haben (Konkurrenzfähigkeit). Gerade Großbritannien investiert im Kontext des Brexit und der Corona-Pandemie mehr in die Entwicklung von Erntehelfern. ${ }^{6}$ Offensichtlich hätte diese Entwicklung großen Einfluss auf die nationale Landwirtschaft (Beschäftigungseffekte, Konkurrenzfähigkeit im globalen Kontext), aber auch Potential zur Steigerung der Ressourceneffizienz und Nachhaltigkeit durch gezielteren Einsatz von Pestiziden/Herbiziden. Im Zusammenhang mit der Einführung dieses Technologiebündels, gibt es freilich einige noch ungeklärte Fragen zur Haftung bei Unfällen von autonomen Robotern und zum Datenschutz.

Die vom Bundesministerium für Nachhaltigkeit und Tourismus (BMNT) eingerichtete Plattform „Digitalisierung in der Landwirtschaft“ hat sich zum Ziel gesetzt, den Einsatz digitaler Technologien in der Landwirtschaft zu verstärken. Das BMNT hat dazu im September 2018 einen Bericht veröffentlicht (BMNT 2018), in dem Handlungsfelder analysiert und bereits erste Risiken und Chancen evaluiert werden. Um das enorme Potenzial der Digitalisierung greifbar zu machen und praxisnah zu veranschaulichen,

1 tab-beim-bundestag.de/de/untersuchungen/u30700.html.

2 idtechex.com/research/reports/agricultural-robots-and-drones-2017-2027technologies-markets-players-000525.asp; blog.robotiq.com/top-10-robotic-applications-in-the-agricultural-industry.

3 zeit.de/2017/14/autonomes-fahren-traktor-acker-landwirtschaft/komplettansicht.

4 derstandard.at/story/2000100231154/obacht-traktor-jetzt-kommen-die-roboter; nytimes.com/2020/02/13/science/farm-agriculture-robots.html.

5 modernfarmer.com/2020/02/futuristic-farming-has-arrived-with-weeding-robots/.

6 derstandard.at/story/2000120213911/roboter-sollen-fehlende-erntehelfer-nachbrexit-ersetzen. 
plant das BMNT zudem einen digitalen Muster-Bauernhof („Innovation Farm") nach dem Vorbild von Deutschland und der Schweiz. ${ }^{7}$

Unter anderem mit dem Landwirtschaftsroboter FRANC ${ }^{8}$ der TU Wien gibt es auch Entwicklungspotenzial in Österreich. Das österreichische Unternehmen smaXtec gilt als eines der führenden Unternehmen bei der Erfassung von Körperdaten von Milchkühen. ${ }^{9}$

\section{Zitierte Literatur}

BMNT (2018) Digitalisierung in der Landwirtschaft Entwicklung, Herausforderungen und Nutzen der neuen Technologien für die Landwirtschaft. Bundesministerium für Nachhaltigkeit und Tourismus: Wien, September, bmnt.gv.at/service/publikationen/land/digitalisierungin-der-landwirtschaft.html.

7 ots.at/presseaussendung/OTS_20181115_OTS0081/koestinger-erster-digitalermuster-bauernhof-es-ist-unsere-aufgabe-digitalisierung-greifbar-zu-machen.

8 franc.acin.tuwien.ac.at.

9 smaxtec.com. 


\section{Genome editing [CRISPR/Cas9] in der Pflanzenzucht}

Genome Editing bezeichnet neue Methoden, die es erlauben, zielgerichtete Eingriffe im Erbmaterial, dem Genom einer Zelle durchzuführen. Zukünftig können damit viele Bereiche der Grundlagenforschung beeinflusst werden und Anwendungen in der Medizin und darüber hinaus möglich werden. Die Anwendung wird für die Pflanzenzüchtung als vielversprechend gesehen. Insbesondere die Methode CRISPR/Cas9 ${ }^{1}$ (siehe auch Thema „Künstliches Leben“), die genutzt wird, um Gene zielgerichtet zu verändern, steht im Mittelpunkt der wissenschaftlichen und öffentlichen Diskussion. Es geht darum, die Erbinformation zu verändern, indem mittels CRISPR/Cas9 einzelne DNA-Bausteine ausgetauscht, entnommen oder hinzugefügt werden. In Bezug auf Pflanzenzüchtung war umstritten, ob CRISPR/Cas9 als eine Form gentechnischer Veränderung zu behandeln ist oder aber als neue Züchtungsmethode. ${ }^{2}$ Im Juli 2018 entschied der Europäische Gerichtshof (EuGH), dass mithilfe des CRISPR/Cas9-Verfahrens entwickelte Pflanzen nur mit einer gentechnikrechtlichen Genehmigung in die Umwelt freigesetzt oder in Verkehr gebracht werden dürfen. ${ }^{3}$ Die Kontroverse um mögliche Potentiale des CRISPR/Cas9-Verfahrens in der zukünftigen Pflanzenzüchtung geht allerdings weiter, da auch gentechnikkritische Akteure CRISPR/Cas9-Verfahren zum Teil als Möglichkeit sehen, um z. B. Nutzpflanzen an den Klimawandel anzupassen, und es mehr Akteuren als den großen Agrokonzernen ermöglichen würde, widerstandsfähigerer Sorten zu entwickeln. ${ }^{4}$

Für die weitere Entwicklung in Europa ist es zum einen notwendig, in der Grundlagenforschung auf europäischer Ebene zu kooperieren, zum anderen Mechanismen zu entwickeln, die eine verantwortungsvolle Forschung und Innovation gewährleisten.

\footnotetext{
1 Als Crispr-Cas9 (kurz für: Clustered regularly interspaced short palindromic repeats- Cas9-System) wird die neue gentechnische Methode bezeichnet, die es ermöglicht, Gene an einer bestimmten Stelle ein- oder auszuschalten.

2 bfr.bund.de/cm/343/fragen-und-antworten-zum-genome-editing-und-crisprcas9.pdf. mpg.de/9943004/gen-editierte-pflanzen.

3 curia.europa.eu/jcms/upload/docs/application/pdf/2018-07/cp180111de.pdf.

4 Siehe zu den Kontroversen, die dem Urteil folgten, z.B. science.orf.at/stories/2934627/; biooekonomierat.de/fileadmin/Publikationen/berichte/BOER-Memo_Genome-Editing.pdf; heise.de/tp/features/Beschleunigte-Evolution-fuer-den-oekologischen-Landbau3928725.html?seite=all; derstandard.at/2000090027602/Protest-gegen-EuGHUrteil-CRISPR-fuer-Grosskonzerne.
} 


\section{Treibstoffe aus Sonnenlicht: Künstliche Photosynthese und bionische Blätter}

Die natürliche Photosynthese ist einer der wichtigsten Prozesse zur Produktion von Biomasse in der Natur. Das Wissen über diesen Mechanismus machen sich Wissenschaftsteams weltweit zunutze und erforschen unter dem Begriff „künstliche Photosynthese“ Möglichkeiten zur Herstellung von $\mathrm{CO}_{2}$-neutralen Biokraftstoffen aus Sonnenlicht (z. B. Wasserstoff).

Dieser Ansatz verspricht mehrere Vorteile in Anbetracht des weltweit steigenden Energiebedarfs. Die Sonne liefert als Energiequelle in einer Stunde mehr Energie zur Erde als wir derzeit an fossiler, nuklearer und erneuerbarer Energie pro Jahr nutzen (Barber/Tran 2013). Die künstliche Photosynthese ermöglicht es, Sonnenlicht direkt in chemische, lagerbare Kraftstoffe (z. B. Wasserstoff, Methan, Ethanol) umzuwandeln, während bspw. Photovoltaik, Windkraft und Erdwärme Strom erzeugen, dessen kostengünstige Speicherung noch nicht gelöst ist (Charisius 2017).

Erste Prototypen sind sogenannte „künstliche Blätter", die die Form von Beutel, Luftpolsterfolie oder auch gewöhnlicher Solarzellen haben können (Rüschemeyer 2017). Diese bionischen Blätter sind in der Produktion von Biomasse effizienter als echte Blätter und stellen den ersten Schritt in Richtung einer einfachen, kostengünstigen und autarken Energieversorgung dar (Nocera 2012).

Während die künstliche Photosynthese als technologische Entwicklung bereits Realität ist, bleibt die Frage ihrer (industriellen) Anwendbarkeit offen. Obwohl der Vorteil künstlicher Photosynthese im dezentralen Einsatz z. B. in Entwicklungsländern ohne zentrale Energieversorgungssysteme gesehen wird, bietet das Forschungsgebiet gerade für die österreichische Grundlagenforschung relevante Anknüpfungspunkte, z. B. bei der Untersuchung von Quanteneffekten in biologischen Systemen (Lim et al. 2015). Aktuell beteiligt sich die Universität Wien an einem europäischen Forschungsprojekt zur Entwicklung künstlicher Chloroplasten1.

\section{Zitierte Quellen}

Barber, J. und Tran, P. D., 2013, From natural to artificial photosynthesis, Journal of The Royal Society Interface 10(81), 20120984.

Charisius, H., 2017, Sprit aus Licht; Süddeutsche.de; sz.de/1.1124182.

Lim, J. et al., 2015, Vibronic origin of long-lived coherence in an artificial molecular light harvester, Nature communications 6, 7755.

Nocera, D. G., 2012, The artificial leaf, Accounts of Chemical Research 45(5), 767-776.

Rüschemeyer, G., 2017, Künstliche Photosynthese. Pack die Sonne in den Tank; Frankfurter Allgemeine Zeitung; faz.net/-gx5-908np.

1 catalight.eu. 


\section{Häuser aus dem 3D-Drucker}

Beim 3D-Druck wird das gewünschte Bauteil schichtweise d. h. additiv hergestellt, wobei unterschiedlichste Materialen zum Einsatz kommen können (Keramik, Kunststoff, Zellen, Metall, Beton etc.). Eine bereits marktreife Anwendung ist das "Drucken“ von Gebäuden. Ein Roboterarm wird in der Mitte oder um das zu errichtende Gebäude positioniert. Dieser Roboterarm verfügt über eine Betongießvorrichtung an seiner äußersten Spitze, mit welcher die Außen- und Innenwände Schicht für Schicht aufgebaut werden können. Diese Technologie ermöglicht es, ein eingeschossiges Gebäude mit $100 \mathrm{~m}^{2}$ Grundfläche innerhalb von 24 Stunden fertigzustellen. Weitere Vorteile, die angegeben werden, sind die drastische Reduktion der Bauabfälle, Kostenersparnis und damit leistbares Wohnen sowie weniger Unfälle im Baugewerbe. Zwei zu nennende Akteure im Bereich des Gebäudedrucks sind das Unternehmen Apis Core ${ }^{1}$ und Contour Crafting Corporation, an welchem unter anderem das in Österreich ansässige doka-Venture mit $30 \%$ beteiligt ist und sich als Kernaktionär positioniert. ${ }^{2}$

Während vor einigen Jahren die ersten Prototypen von Gebäuden mittels 3D-Druck errichtet wurden, ${ }^{3}$ entstehen 2019 zunehmend kommerzielle Projekte. Beispielweise werden in Eindhoven (NL) fünf bewohnbare Betonhäuser realisiert. ${ }^{4}$ In Lateinamerika soll ein Siedlungsprojekt mit mehr als 50 Häusern für Geringstverdiener entstehen. ${ }^{5}$ In Nantes (FR) ist ein erstes 3D gedrucktes Haus bereits bewohnt. ${ }^{6}$ In Belgien wurde 2020 das erste gedruckte Niedrigenergiehaus Europas gebaut. ${ }^{7}$ Das derzeit größte gedruckte Haus ist mit $641 \mathrm{~m}^{2}$ ein in nur 17 Tagen errichtetes Bürogebäude in Dubai. ${ }^{8}$ Diese neuartige Anwendung der additiven Fertigung könnte ein disruptives Potential für das Bauwesen und der Beschäftigung in diesem Bereich haben. Bezogen auf Sicherheit und Baunormen, ist bei einer zunehmenden Verbreitung der Technologie von Handlungsbedarf auszugehen. Diese Technologie ermöglicht zudem die Schaffung leistbaren und vor allem schnell errichteten Wohnraums, was sie aus Sicht der Wohnbaupolitik relevant macht.

\footnotetext{
1 apis-cor.com/en/.

2 3d-grenzenlos.de/wp/wp-content/uploads/2017/06/doka-ventures-3d-druckhaeuser.pdf.

3 youtube.com/watch?v=xktwDfasPGQ.

4 archdaily.com/tag/houben-and-van-mierlo.

5 fastcompany.com/90317441/there-will-soon-be-a-whole-community-made-ofthese-ultra-low-cost-3d-printed-homes.

6 computerwelt.at/news/topmeldung/weltpremiere-erste-familie-zieht-in-3d-druckhaus/.

7 ingenieur.de/technik/fachbereiche/3d-druck/europas-erstes-niedrigenergiehausaus-dem-3d-drucker/.

8 pcwelt.de/news/Weltrekord-641-Quadratmeter-Haus-aus-dem-3D-Drucker10751031.html.
} 


\section{Dienstleistung 4.0}

Die Digitalisierung der Dienstleistungsarbeit ermöglicht es „virtuellen ArbeiterInnen" für Arbeitgeber in anderen Ländern zu arbeiten. Damit stehen österreichische ArbeitnehmerInnen im Wettbewerb mit AnbieterInnen aus allen Teilen der Welt, mit zum Teil wesentlich niedrigerem Lohnniveau und geringer sozialer Sicherheit (Lutz und Risak 2017). Online-Plattformen überwinden Firmen-, sektorale und nationale Grenzen und öffnen globale Märkte für mehr Wettbewerb bei weniger Regulierung (ITA 2017). Es entstehen Plattformen, die das Auffinden virtueller Arbeit ermöglichen bzw. diese vermitteln. ${ }^{1}$ Reputationssysteme verfolgen individuelle Leistungen, Kompetenzen und spezifische Fähigkeiten, damit virtuelle Arbeitgeber schnell kompetente und vertrauenswürdige MitarbeiterInnen finden können. Online-Plattformen wie Uber oder AirBnB machen mit insgesamt sehr wenigen direkt Beschäftigten hohe Umsätze.

Neben den Crowdworking-Plattformen sind es vor allem die Verlagerung von Dienstleistungen auf die KonsumentInnen (prosumer), die hohe Einsparungspotentiale seitens der Unternehmen ermöglichen, und der zunehmende Einsatz von Robotern und Algorithmen, die auch höherwertige Dienstleistungsarbeit übernehmen bzw. automatisieren können. Damit zeichnen sich einschneidende strukturelle Veränderungen in Bereichen wie Handel, Bank- und Versicherungswesen sowie in Pflege- und Gesundheitsdienstleistungen ab.

Eine EU-Richtlinie über transparente und vorhersehbare Arbeitsbedingungen in der Europäischen Union wurde im vergangenen Jahr verabschiedet und ist bis Mitte 2022 in nationales Recht umzusetzen. ${ }^{2}$ Bereits jetzt gibt es Kritik, dass die Richtlinie nur teilweise zu einer Verbesserung der Situation führt und zu ungenau ist; gerade die Rechte der Arbeitnehmerlnnen in der sog. „Gig-Economy“ werden nicht ausreichend gestärkt. ${ }^{3}$

Hierzu hat das österreichische Parlament Handlungsspielraum bei der Umsetzung der EU-Richtlinie und kann so auf Probleme eingehen. Hierbei sind die oben erwähnten strukturellen Änderungen am Arbeitsmarkt und in der Unternehmensstruktur in Österreich zu beachten. Diese haben Auswirkungen auf das Steueraufkommen und die Steuerpolitik. Dazu kommen Fragen der Anpassung von Arbeitsrecht und Schutz von EPUs, Sozialversicherungsfragen und nicht zuletzt ethische Fragen bei Pflege- und Gesundheitsdienstleistungen (siehe auch Lutz und Risak 2017).

\footnotetext{
1 clickworker.com und crowdguru.de.

2 Richtlinie 2019/1152 vom 20.06.2019.

3 lesen.lexisnexis.at/_/schoeffmann-die-neue-transparenzrichtlinie-zas-2019244/artikel/ard/2020/6682/ARD_2020_6682_014.html.
} 


\section{Zitierte Literatur}

ITA 2017, Roboter, Digitalisierung und Arbeitsmarkt. ITA-Dossier Nr. 26, April; Tanja Sinozic, Michael Nentwich, Johann Čas), Wien; epub.oeaw.ac.at/ita/ita-dossiers/ita-dossier026.pdf.

Lutz, Doris, and Martin E. Risak, eds. 2017. Arbeit in Der Gig-Economy:

Rechtsfragen Neuer Arbeitsformen in Crowd Und Cloud. Varia. Wien: ÖGB Verlag, Verlag des Österreichischen Gewerkschaftsbundes. 


\section{Automatisierung in der Rechtsberatung}

Die Digitalisierung, Automatisierung und künstliche Intelligenz findet in vielen verschiedenen Bereichen Einzug. Einer davon ist der Rechtsbereich. Diese so genannten "Legal-Techs" umschreiben Technologien, die von unterstützender Software im Büroalltag bis hin zu automatisierten Rechtsdienstleistungen und smart contracts reichen, also Verträgen die in eine Blockchain eingebettet sind. Aktuelle Anwendungsbeispiele sind Onlineportale, die sich auf Flugentschädigungen spezialisiert haben (Compensation2Go, Airhelp, refund.me etc.). Hierbei werden für die Kunden die Entschädigungen beim Anbieter erwirkt. Technisch aufwändiger sind Technologien, die die Recherche und das Verfassen von Schriftsätzen übernehmen. Das funktioniert durch die algorithmische Einbettung von juristischem Regelwerk. Auch das österreichische Patentamt nutzt bereits eine Form der algorithmischen Rechtsberatung (Markenschutz). Der „Albert Patent Bot" unterstützt Unternehmen dabei, markenrechtliche Fragen online abzuklären. Laut einer Studie der Boston Consulting Group und der Bucerius Law School könnten die Effekte des Einsatzes von Legal-Techs weitreichend sein, unter anderem wird davon ausgegangen, dass 30-50\% der Arbeiten, die Anwaltsanwärterlnnen heute noch verrichten, dadurch substituiert werden könnten. ${ }^{1}$ Der Einfluss auf den Arbeitsmarkt und auf die Ausbildung von Juristlnnen wäre demnach nicht zu vernachlässigen. Für die KonsumentInnen ergäben sich Vorteile, wie zum Beispiel der niederschwellige Zugang zu Rechtsdienstleistungen. Rechtsprechung und Streitschlichtung sind derzeit noch keine Schwerpunkte von Legal Tech, die prinzipiell aber auch Anwendung für die Arbeit an Gerichten finden können, z. B. bei der automatischen Bearbeitung gleichförmiger Streitfälle. Die (Teil-)automatisierte Onlinestreitschlichtung (online dispute resolution) könnte dabei zum Vorbild werden; hierkönnen Rechte aus Onlinegeschäften durch eine privatrechtlich organisierte Schlichtungsstelle ohne Einbezug der Judikative geltend gemacht werden (Kind et al. 2019). Weitestgehend ungeklärt sind die Implikationen des algorithmischen Eingriffs in die Rechtsstaatlichkeit und inwieweit dieser überhaupt automatisiert werden soll? ${ }^{2}$ Auch die Frage der Sicherstellung des Datenschutzes bei der Algorithmisierung im Rechtsbereich muss berücksichtigt werden.

Laut einer Umfrage von LexisNexis im Jahr 2017, stehen Österreicherlnnen der automatisierten Rechtsberatung eher offen gegenüber. Immerhin $68 \%$ der Befragten ( $n=500)$ könnten sich vorstellen, solche Dienstleistungen in Anspruch zu nehmen (LexisNexis 2017). Eine grundlegende Akzeptanz der österreichischen Bevölkerung gegenüber Legal-Techs scheint demnach wahrscheinlich. Für österreichische Rechtsanwaltskanzleien mit ein bis drei PartnerInnen könnten Legal-Techs vor allem im Bereich der

\footnotetext{
1 bucerius-education.de/fileadmin/content/pdf/studies_publications/Legal_Tech_ Report_2016.pdf.

2 tab-beim-bundestag.de/de/pdf/publikationen/themenprofile/Themenkurzprofil012.pdf.
} 
Dokumentenerstellung (z. B. Vertragserstellung oder Dokumentenprüfungsservices) zur Konkurrenz werden (LexisNexis 2017). Ein Großteil der in Österreich tätigen Rechtsanwaltskanzleien wäre dadurch betroffen, da ca. $86 \%$ Kanzleien aus wenigen PartnerInnen bestehen (ÖRAK 2018). In Österreich wurde deshalb jüngst der so genannten Legal Tech Hub durch sieben bedeutende Wiener Rechtsanwaltskanzleien gegründet. Ihr Ziel ist es, Kompetenzen im Bereich der Legal Techs aufzubauen, Entwicklungen zu fördern und bei der Entwicklung von Standards mitzuwirken. ${ }^{3}$ Diese Entwicklungen zeigen, dass der Berufsstand der Rechtsanwälte bereits auf die Veränderungen reagiert und anpasst. Unklar bleibt jedoch nach wie vor, welchen Einfluss die Digitalisierung des Rechtsbereichs auf den Rechtsstaat selbst hat.

\section{Zitierte Literatur}

LexisNexis Whitepaper, 2017, Digitalisierung der Rechtsbranche Teil I von II, Verlag NexisLexis: lexisnexis.at/vie/pdf/LexisNexis-WhitepaperDigitalisierung-Rechtsbranche.pdf.

Österreichische Rechtsanwaltskammer (ÖRAK), 2018, Gemeinsam für den Rechtsstaat - Tätigkeitsbericht 2018, Wien:

rechtsanwaelte.at/fileadmin/user_upload/PDF/02_Kammer/Stellungnah men/Taetigkeitsbericht/tb_2018.pdf.

Sonja Kind, Jan-Peter Ferdinand, Kai Priesack (2019) Legal Tech Potenziale und Wirkungen. TAB-Arbeitsbericht Nr. 185, Büro für Technikfolgen-Abschätzung beim Deutschen Bundestag. tab-beimbundestag.de/de/pdf/publikationen/berichte/TAB-Arbeitsberichtab185.pdf.

3 derstandard.at/2000090746497/Sieben-Kanzleien-ziehen-an-einem-Strang. 


\section{Die Zukunft von Industrie 4.0}

Es besteht kein Zweifel, dass die Entwicklung zur Industrie 4.0 (cyberphysical systems) weiter voranschreiten wird: Roboter werden multifunktional, intelligenter, kleiner, billiger und sicherer, sodass diese in Zukunft auch außerhalb von strikten Sicherheitsbereichen (sog. Käfigen) in der Montagehalle gemeinsam mit Menschen "Hand in Hand“ arbeiten werden. Vor allem der Trend zu kleineren, multifunktionalen und billigeren Robotern wird auch die KMUs erreichen. Gleichzeitig schreitet die Entwicklung des Internet der Dinge (IoT) voran, was zu einer virtuellen Verkettung der gesamten Produktionskette, vom Rohstoff über alle Zwischenprodukte und Lieferanten bis zum Fertigprodukt, führen kann - mit entsprechenden Auswirkungen auf die traditionellen Logistiksysteme und auch die Produktionsverfahren. Bisher wurden vor allem die Auswirkungen auf den Arbeitsmarkt diskutiert, dies jedoch weiterhin noch ohne eindeutige Ergebnisse. Darüber hinaus ergeben sich mannigfache Wirkungen, die die möglichen Veränderungen der Wirtschaftsstruktur ebenso umfassen, wie Auswirkungen auf die erforderlichen Qualifikationen und damit auf das Bildungssystem - so werden beispielsweise Anpassungen der Lehrinhalte technischer Studiengänge empfohlen (Kamble et al. 2018). Insbesondere das Zusammenwirken von Menschen und Robotern bedarf noch sozialwissenschaftlicher und ethischer Überlegungen.Das BMVIT investiert in F\&E in diesem Bereich. Als Ort, der "wichtige gesellschaftliche, politische, wirtschaftliche und wissenschaftliche Akteure an der Gestaltung der zukünftigen Produktions- und Arbeitswelt" vereint, wurde die Plattform Industrie 4.0 gegründet. ${ }^{1}$ Für die Forschung an konkreten Umsetzungsthemen und für den intensivierten Wissenstransfer wurde eine Pilotfabrik eingerichtet. ${ }^{2} 14.0$ scheint gerade in Österreich ein boomender Bereich mit großen Chancen. Mit der Pilotstudie Industrie 4.0 (Aichholzer et al. 2015) hat das Parlament 2015 einen ersten umfassenden Überblick über die vielfältigen Auswirkungen von Industrie 4.0 bekommen, es wurden damals aber nur zwei von neun Wirkungsfeldern ${ }^{3}$ vertieft. International wurde bereits auf die Zweischneidigkeit von Industrie 4.0 verwiesen, die (neben weiteren Vor- und Nachteilen) beispielsweise sowohl zu besserem Informationsfluss über Produkte und deren Entstehung als auch zu Verletzungen von Persönlichkeitsrechten führen kann (Oztemel und Gursev 2018). Dies offenbart auch eine große Schwierigkeit der Abschätzung von 14.0: Viele Akteure aus Industrie und Wissenschaft forschen und entwickeln an unterschiedlichen Bereichen von 14.0 und noch ist wenig vereinheitlicht (ibid. 2018). Eine weiterführende Studie, die auch die weiteren Wirkungsfelder miteinbezieht, kann hier für mehr Klarheit sorgen.

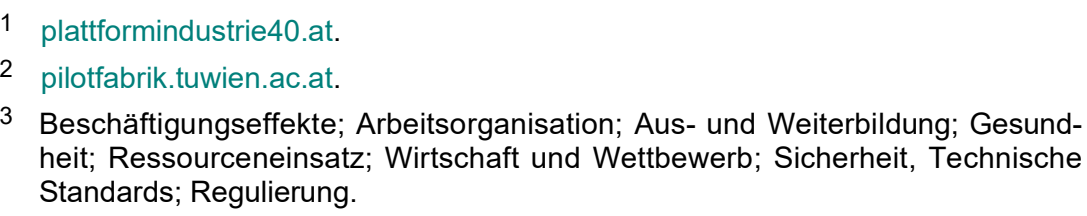

3 Beschäftigungseffekte; Arbeitsorganisation; Aus- und Weiterbildung; Gesundheit; Ressourceneinsatz; Wirtschaft und Wettbewerb; Sicherheit, Technische Standards; Regulierung. 


\section{Zitierte Quellen}

Aichholzer, G., Gudowsky, N., Rhomberg, W., Saurwein, F., Weber, M. und Wepner, B., 2015, Industrie 4.0 - Foresight \& Technikfolgenabschätzung zur gesellschaftlichen Dimension der nächsten industriellen Revolution (Zusammenfassender Endbericht), Nr. ITA-AIT-2, 2015-11-30, Wien; epub.oeaw.ac.at/ita/ita-projektberichte/ITA-AIT-2.pdf.

Gawankar, S., Gunasekaran, A., Kamble, S., 2018, Sustainable Industry 4.0 framework: A systematic literature review identifying the current trends and future perspectives, in: Process Safety and Environmental Protection 117, Juli 2018, 408-425, doi.org/10.1016/j.psep.2018.05.009.

Gursev, S., Oztemel, E., 2018, Literature review of Industry 4.0 and related technologies, in: Journal of Intelligent Manufacturing 31, 2020, 127-182, doi.org/10.1007/s10845-018-1433-8. 


\section{Von +Energie zu ++Energie: Zur Zukunft des Bauens}

Plusenergiegebäude (+Energiegebäude) sind im Kommen. Sie produzieren mehr Energie, als sie verbrauchen, und sind als vereinzelte Leuchtturmprojekte bereits realisiert (z.B. in Österreich „Bürohochhaus TU

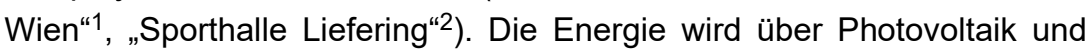
Rückgewinnung (Bremsenergie der Aufzüge, Abwärme aus dem Serverraum etc.) produziert und deckt die Gebäudegrundfunktionen (Heizung, Kühlung, Lüftung, Beleuchtung etc.) ab. Über das Jahr gesehen gibt es einen Energieüberschuss.

Der nächste Schritt in die Zukunft sind ++Energiegebäude. Sie haben den Anspruch, nicht nur den Energiebedarf der Gebäudegrundfunktionen abzudecken, sondern auch den der Nutzung von Gegenständen im Gebäude (von der Kaffeemaschine über den Kühlschrank bis zum Laptop). Für mehrere Geschosse ist dies im Leuchtturmprojekt „Bürohochhaus TU Wien" bereits realisiert.

Das Erreichen eines + bzw. ++Energiestandards ist bei einem mehrstöckigen Gebäude eine besondere Herausforderung, da die Energiegewinnung zu einem großen Teil über Photovoltaik realisiert wird. Jedes zusätzliche Stockwerk müsste durch ein und dieselbe Dachfläche versorgt werden. Ein ++ Gebäude kann daher nicht mit einer einzelnen innovativen Lösung, sondern nur mit einem intelligenten Gesamtkonzept ermöglicht werden, das viele einzelne Komponenten integriert (etwa fassadenintegrierte Photovoltaik, Heizung mit der Abwärme aus dem Serverraum, effiziente Energienutzung durch Steuerung von Licht, Raumwärme, Jalousien etc. über Sensoren, minimierter Stand-by-Strombedarf bei Geräten etc.) (vgl. David \& Bednar 2017).

Einerseits versprechen ++Energiegebäude viele Vorteile und Chancen, z.B. den möglichen Ausgleich von Nutzungsspitzen durch die Produktion von Überschussenergie, die sich vor allem durch die Einbettung von ++Energiegebäuden in energieeffiziente Stadt- bzw. Ortsteile ergibt (siehe Thema Plus Energie Quartiere). Andererseits könnten zukünftig auch Nachteile mit den auf - oftmals proprietären - digitalen Technologien beruhenden und zur Steigerung der Energieeffizienz geforderten „intelligenten Gesamtlösungen" für ++Energiegebäude einhergehen, v.a. wenn sie für privates Wohnen genutzt werden. In Anbetracht eines boomenden, globalen „Smart Home“3-Marktes, dem für den Zeitraum 2017-2022 jährli-

\footnotetext{
1 tuwien.at/tu-wien/campus/tu-univercity/standorte/plus-energie-buerohochhaus/.

2 stadt-salzburg.at/internet/bildung kultur/sport und erholung/sporthallen/ sportzentrum_nord_li_450430/sportzentrum_nord_liefering_450424.htm.

3 Smart Home bezeichnet Heiminformationssysteme, die anhand von digitalen Sensoren und Geräten die kombinierte Analyse von Umweltinformation (z.B. Lichtverhältnisse, Raumwärme) und von persönlicher Information der BewohnerInnen (z.B. Körpertemperatur, Bewegungsprofile) ermöglichen und auf die Steigerung des Wohlbefindens und der Lebensqualität im Heimbereich durch Automatisierung abzielen.
} 
che Wachstumsraten von $47 \%$ vorhergesagt wurden, ${ }^{4}$ gewinnt die Frage nach der Datenhoheit über die in den ++Energiegebäuden zukünftig gesammelten Informationen an Bedeutung (siehe Themen Digitalisierung und Anonymität; Cybersicherheit). Wenn ++Energiestandards ein zentrales Element von „Bauen im 21. Jahrhundert" sein sollen, ist die Bearbeitung von damit verbundenen Problemstellungen, wie der langfristigen Garantie von Privatsphäre und Datensicherheit in ++Energiegebäuden und intelligenten Gebäudesystemen, Voraussetzung für eine hohe, gesellschaftliche Akzeptanz.

\section{Zitierte Literatur}

David, A., Leeb, M. und Bednar, T. (2017) Comparison of the planned and the real energy consumption of the world's first (Plus-)Plus-Energy Office High-Rise Building. Energy Procedia (132), 543-548.

4 bcg.com/publications/2018/mapping-smart-home-market.aspx. 


\section{Peer-to-Peer[P2P]-Energiehandel}

Ein neues Paradigma in der Stromversorgung ist der Peer-to-Peer (P2P)Energiehandel. Eine Schlüsselidee beim P2P-Energiehandel ist die Transformation von KonsumentInnen in ProsumentInnen, die nicht nur Energie konsumieren, sondern auch produzieren. Die Ausgangsbasis für den P2P-Energiehandel sind lokale EnergieprosumentInnen, die erneuerbare Energiequellen (z.B. Solaranlagen, Geothermie) in Wohnanlagen, Bürohäusern, Fabriken o.ä. nutzen und damit eine dezentrale Energieversorgung ermöglichen (Zhang et al. 2017). Unterschiedliche Aspekte spielen in der Etablierung des P2P-Energiehandels weltweit eine Rolle: Geschäftsmodelle, Plattformlösungen sowie Informations- und Kommunikationstechnologien (Cloud-Services, Blockchain) (Zhang et al. 2017). Die Zukunftsvision für den P2P-Energiehandel ist ein von zentralen Instanzen unabhängiges Energiesystem, das dezentrale Geschäftsmodelle fördert und ohne Zwischenhändler auskommt (Hasse et al. 2016).

Ein dezentrales Energiesystem besteht aus mindestens zwei Ebenen: Die untere Ebene bilden lokale Inselnetze (Microgrids), die autark Strom aus erneuerbaren Quellen erzeugen und an ein übergeordnetes Netz angeschlossen werden können (Supergrids). Die darüber liegende Ebene ist ein funktionierendes IKT-System, das Stromversorgung, Kontrolle/Monitoring und Handel ermöglicht. Diese Ebene wird meist über eine Handelsplattform implementiert, über die Energie angeboten und gekauft werden kann. Die Konzeption einer solchen Plattform bestimmt die Möglichkeiten des Handels mit (Zhang et al. 2018). Sie sorgt für Informationsaustausch zwischen KäuferInnen und Verkäuferlnnen sowie für Kontrolle über die tatsächliche Stromversorgung. Die Einführung bestimmter Handelsprinzipien beeinflusst die Entwicklung der aufgrund lokaler Wetterereignisse und Bedarfsschwankungen ohnehin schwer einschätzbaren dezentralen Märkte zusätzlich.

Dezentrale Energiemärkte können sowohl mithilfe von Blockchain-Technologie, als auch über alternative Lösungen realisiert werden (Hasse et al. 2016). Bisher existieren keine umfassenden Blockchain-Anwendungen im Energiehandel. Einige Pilotprojekte wurden in den USA und in den Niederlanden umgesetzt. ${ }^{1}$ Auch in Österreich erforschen erste Projekte zu Blockchain in der Energiewirtschaft die Möglichkeiten von neuen und effizienten Lösungen für Energiemanagement-Services und Energiehandel. ${ }^{2}$ Das Potenzial von Blockchain im P2P-Energiehandel ist vielversprechend. Für die Informationsdokumentation auf einer P2P-Handelsplattform ermöglichen Blockchain-Technologien die einfache, flächendeckende Ar-

1 Brooklyn Microgrid (TransactiveGrid) brooklyn.energy; Vattenfall: Powerpeers (Niederlande) powerpeers.nl/about; RWE und slock.it: Blockcharge; Oneup: POWR oneup.company; LO3 Energie: Exergy projectexergy.com.

2 Projekt SonnWende+ nachhaltigwirtschaften.at/de/sdz/projekte/sonn-wendeplus.php, für weitere Beispiele siehe: smartgrids.at/files/smartgrids/Dateien/ Dokumente/Dokumente/Fact\%20Sheet\%20Blockchain.pdf. 
chivierung aller Abrechnungsdaten des Stromverbrauchs. Die dezentrale Speicherung von Transaktionsdaten durch Blockchain-Technologie (siehe dazu Thema „Vertrauenswürdige Blockchains“) kann wiederrum das Sicherheitsniveau erhöhen, die Unabhängigkeit von einer zentralen Instanz garantieren und die Implementierung dezentraler Geschäftsmodelle erleichtern, v.a. durch Smart Contracts (Hasse et al. 2016). Im Unterschied zum Finanzsektor ist bei Blockchain-Anwendungen im Energiehandel das physische Produkt (der Stromfluss) zu berücksichtigen.

Ob sich Blockchain als zentrale Technologie für P2P-Energiehandel durchsetzen wird, hängt stark von der zukünftigen Entwicklung der bestehenden Energieinfrastruktur ab (z.B. Smart Meter Rollout, Datensicherheit etc.). Ebenso beeinflussen regulatorische Rahmenbedingungen, Skalierbarkeit und Resilienz der Technologie sowie die Wirtschaftlichkeit der Investitionen den zukünftigen Erfolg der Technologie. Daher sind Energiepolitik, Regulierung und die aktuelle sowie zukünftig angestrebte Struktur des Versorgungssystems entscheidend für die Realisierung von Effizienzpotenzialen des Peer-to-Peer-Energiehandels.

\section{Zitierte Quellen}

Hasse, F., Von Perfall, A., Smole, E., Lay, L. und Charlet, M., 2016, Blockchain - Chance für Energieverbraucher?, im Auftrag von: PwC, 26. Juli 2016, Düsseldorf.

Zhang, C., Wu, J., Long, C. und Cheng, M., 2017, Review of Existing Peer-toPeer Energy Trading Projects, Energy Procedia 105, 2563-2568.

Zhang, C., Wu, J., Zhou, Y., Cheng, M. und Long, C., 2018, Peer-to-Peer energy trading in a Microgrid, Applied Energy 220, 1-12. 


\section{Pflanzen als vernetzte Umweltsensoren}

Sensoren spielen heutzutage eine wichtige Rolle in verschiedenen Steuerungs-und Regelkreisen. Ihre Bedeutung wird innerhalb von intelligenten und vernetzten Städten vermutlich sogar größer werden. In diesem Zusammenhang besteht die Idee, sich Pflanzen als so genannte Biosensoren zu Nutze zu machen. Jede Pflanze besitzt eine Unzahl an biologischen Mechanismen, die es ihr ermöglichen, bestimmte Umweltparameter wie Luftfeuchtigkeit, Temperatur, $\mathrm{CO}_{2}$-Gehalt, Licht etc. zu messen. Die Signalverarbeitung in Pflanzen erfolgt über elektrische Signale. Die technologische Innovation bestünde nun darin, diese "Messinstrumente“ auf entsprechende Art und Weise zu nutzen. Über eine technische Schnittstelle könnten die pflanzlichen Daten „ausgelesen“ und dann drahtlos an die Messzentrale übermittelt werden. Damit wäre ein innovatives und engmaschiges Umwelt-Monitoring möglich. Das konnte bereits in ersten Versuchen nachgewiesen werden. Mit diesem neuartigen Ansatz können Schadstoffauswirkungen, wie zum Beispiel von Pestiziden oder die Vorhersage und Beobachtung von klimatischen Veränderungen nachgewiesen werden (Volkov/Ranatunga 2006). ${ }^{1}$ Pflanzen können demnach als Umweltsensoren eingesetzt werden, indem man über eine spezielle Schnittstelle die Pflanze mit einem Computer verbindet. Die dazu notwendige Technologie ist zwar noch nicht marktreif, jedoch konnte durch den Demonstrationsaufbau der "proof of concept“ erbracht werden. ${ }^{2}$ Unter anderem gibt es auch Fortschritte beim Einsatz von Nanomaterialien für Biosensoren, die z.B. helfen können Pflanzenkrankheiten früh zu erkennen (Kundu et al. 2019).

Die erfolgreich durchgeführten Experimente eröffnen viele neue Möglichkeiten. Der Einsatz in der Landwirtschaft würde die Beobachtung von ernterelevanten Faktoren ermöglichen. Eine italienische Forschungsgruppe hat einen biomimetischen, textilbasierten Sensor entwickelt, der es erlaubt, physiologische Schlüsselparameter einer Paradeiserpflanze zu monitoren. Die Autorlnnen der Studie sehen in dieser Technologie ein großes Potential für das Precision Farming (siehe Thema „Robotik in der Landwirtschaft") (Coppedè et al. 2017). Auf ähnliche Weise könnten solche Sensoren auch für das Monitoring der Luftqualität oder der Schadstoffbelastung in Städten genutzt werden. Eine Anwendung für das KlimaMonitoring scheint ebenfalls vielversprechend zu sein.

\section{Zitierte Quellen}

Volkov, A. G. und Ranatunga, D. R. A., 2006, Plants as Environmental Biosensors, Plant Signaling \& Behavior 1(3), 105-115, ncbi.nlm.nih.gov/pmc/articles/PMC2635006/.

\footnotetext{
1 Das wurde auch im Zuge eines EU-Projekts bestätigt: PLants Employed As SEnsor Devices (PLEASED); cordis.europa.eu/project/rcn/103686_en.html.

2 youtube.com/watch?v=D2sjmLbT6NY.
} 
Coppedè, N., Janni, M., Bettelli, M., Maida, C. L., Gentile, F., Villani, M., Ruotolo, R., lannotta, S., Marmiroli, N., Marmiroli, M. und Zappettini, A., 2017, An in vivo biosensing, biomimetic electrochemical transistor with applications in plant science and precision farming, Scientific Reports 7(1), 16195, doi.org/10.1038/s41598-017-16217-4.

Kundu, M., Krishnan, P., Kotnala, R. K. und Sumana, G., 2019, Recent developments in biosensors to combat agricultural challenges and their future prospects, Trends in Food Science \& Technology 88, 157-178, sciencedirect.com/science/article/pii/S0924224417307422. 


\section{$\mathrm{CO}_{2}$-neutrale Gebäudekühlung}

Hitzetage werden auch in Österreich immer häufiger. ${ }^{1}$ Damit steigt der Bedarf, Gebäude zu kühlen, um ein angenehmes Raumklima zu erzielen. Der umfassende Einsatz von energieintensiven Technologien zur Gebäudekühlung wie z.B. Klimaanlagen stellt keine nachhaltige Lösung dar, weil der erhöhte Strombedarf im Sommer zu kurzfristig hohen Strompreisen und zu netzbedingten Problemen bis hin zu Blackouts führen kann. ${ }^{2}$ Laut einer Umfrage aus dem Jahr 2019 plant jedeR dritte Österreicherln bzw. sogar jedeR zweite WienerIn den Kauf eines Klimagerätes. ${ }^{3}$ Die Internationale Energie Agentur (IEA) geht davon aus, dass sich global betrachtet der Energiebedarf für die Gebäudekühlung bis zum Jahr 2050 verdreifachen könnte. Sie sieht in dieser Tendenz einen großen energie- und klimapolitischen Handlungsbedarf. Der Trend zum erhöhten Kühlbedarf in Gebäuden ist anhand der steigenden internationalen Verkaufszahlen für Klimageräte bereits jetzt zu erkennen (IEA 2018). Der zusätzliche Strombedarf gefährdet auch die energie- und klimapolitischen Ziele der Bundesregierung (Dekarbonisierung und 100\% erneuerbare Stromversorgung) und konkurriert zudem mit dem zunehmenden Strombedarf für Elektromobilität. Berechnungen zufolge könnte der Strombedarf für Gebäudekühlung mittels herkömmlicher Kühltechnologie in Österreich bis 2030 eineinhalbmal so hoch sein wie der Strombedarf zur Raumheizung. Diesen Trend gilt es rechtzeitig abzufangen und geeignete Gegenmaßnahmen zu setzen. Im Jahr 2007 war der Strombedarf zur Raumheizung etwa zehnmal höher als zur Raumkühlung (EEG 2007).

Aktuell wird in Österreich intensiv an der Erforschung von Alternativen gearbeitet. Erste Pilotprojekte für $\mathrm{CO}_{2}$-neutrale Gebäudekühlung in Österreich sind in der ENERGYbase ${ }^{4}$ und in aspern $\mathrm{IQ}^{5}$ verwirklicht. Diese Systeme kühlen über Erdwärme bzw. Grundwasser. Die saisonale Gleichzeitigkeit von verfügbarer solarer Energie und des Kühlungsbedarfs legt eine verstärkte Nutzung von Technologien nahe, die sich unter dem Begriff des "solaren Kühlens" subsummieren lassen. Das technische Prinzip beruht darauf, dass die solare Energie entweder in Form von Strom oder Wärme genutzt wird, um eine Kältemaschine anzutreiben. In diesen Anlagen werden spezielle Sorptionsmaterialien als Kältemittel eingesetzt (Wasser, Lithium-Bromid, Zeolithe, Siliziumgel etc.) Diese Technologien bringen sowohl technische als auch ökologische Vorteile mit sich. Technisch gesehen würden sie zu einer Minimierung der Sommerspitze führen, die durch den hohen Energiebedarf durch konventionelle

\footnotetext{
1 zamg.ac.at/cms/de/klima/news/ueberdurchschnittlich-viele-heisse-tage.

2 uibk.ac.at/bauphysik/forschung/publications/scopt/ap9_broschuere_solare_kuehlen_2013.pdf.

3 elektro.at/2019/05/08/jeder-dritte-plant-kauf-eines-klimageraetes/.

4 pos-architecture.com/projects/energy-base/.

5 nachhaltigwirtschaften.at/de/hdz/projekte/aspern-die-seestadt-wiens-subprojekt3a-technologiezentrum-aspern-iq.php.
} 
Kühlung entsteht. Sämtliche technische Komponenten sind bereits am Markt verfügbar, wenngleich es noch Optimierungs- und Weiterentwicklungspotentiale gibt. Zu den ökologischen Vorteilen zählen der Verzicht auf den Einsatz von umweltbelastenden Kältemitteln und ein Beitrag zur Reduktion der Treibhausgasemissionen durch die Einsparung fossiler Energieträger im Gegensatz zu konventionellen Gebäudekühlsystemen.

Eine Herausforderung besteht derzeit vor allem darin, die beste Kombination aus bereits verfügbaren Anlagenkomponenten für die entsprechende Anwendung zu konzipieren und umzusetzen. Die österreichische Forschungslandschaft beschäftigt sich seit mehreren Jahren mit dieser Technologie. Es existiert bereits eine österreichische TechnologieRoadmap für die solarthermische Kühlung. ${ }^{6}$ Eine besondere Relevanz dieser Technologie ergibt sich einerseits aufgrund der Einsparungspotentiale bei Treibhausgasen und andererseits aus industriepolitischer Sicht aufgrund des antizipierten Wachstumspotentials dieses Technologiefeldes. Auch alternative technologische Konzepte zur $\mathrm{CO}_{2}$-neutralen $\mathrm{Ge}$ bäudekühlung (siehe Frugale Kühlung), wie etwa über Fassadenbegrünungen, zeigen deutliche Energieeinsparungen (Nguyen et al. 2019).

\section{Zitierte Quellen}

EEG (Haas, R. et al.) 2007, Wärme und Kälte aus Erneuerbaren 2030, Dachverband Energie Klima, Energy Economics Group, Wien.

IEA, 2018, The Future of Cooling, Paris: IEA - International Energy Agency iea.org/reports/the-future-of-cooling.

Nguyen, P. A., Bokel, R. und van den Dobbelsteen, A., 2019, Effects of a Vertical Green Façade on the Thermal Performance and Cooling Demand, Journal of Facade Design and Engineering; Vol 7 No 2 (2019): Facade Design and Engineering superheroscitech.tudelft.nl/jfde/article/view/3819.

6 ait.ac.at/fileadmin/mc/energy/downloads/Endbericht-Publizierbar-Roadmap_SKneu.pdf. 


\section{Kommerzialisierung von Geoengineering-Technologien}

Der Traum, das Wetter zu beeinflussen, ist alt und lange Zeit standen Kulturtechniken wie Opfern und Beten im Vordergrund. Seit Anfang des 20. Jahrhunderts wurden immer mehr technische Ansätze entwickelt, wie beispielsweise die in ihrer Wirksamkeit umstrittene Wolkenimpfung. Dabei werden Kondensationskerne - meist Silber- oder Bleijodid - in der Atmosphäre ausgebracht, um so das kontrollierte Abregnen zu begünstigen. Weitreichende Klimabeeinflussung wird verstärkt im Zuge der Debatte um den menschengemachten Klimawandel diskutiert. Nachdem nationale und internationale Entscheidungsfindung und Umsetzung zur Kontrolle der atmosphärischen Treibhausgaskonzentration langwierig und fragil sind, werden immer wieder auch - sehr umstrittene - großtechnische Handlungsoptionen diskutiert, die weitreichende Folgen hätten (Caviezel/ Revermann 2014). Grundsätzlich lassen sich diese in zwei Kategorien einteilen: erstens Technologien zur Senkung der atmosphärischen $\mathrm{CO}_{2-}$ Konzentration, sogenanntes CDR - Carbon-Dioxide-Removal, oder auch negative-emissions-technologies: z.B. Eisendüngung der Ozeane, um das Wachstum von Algen anzuregen, welche dadurch $\mathrm{CO}_{2}$ aufnehmen und langfristig binden da sie nach dem Absterben auf den Meeresboden sinken; großflächige Aufforstungen; oder auch $\mathrm{CO}_{2}$-Abscheidung beispielsweise in Kraftwerken und Nutzung bzw. dessen Speicherung z.B. in alten Gas- oder Öllagerstätten (Carbon Capture and Storage, CCS). Die zweite Kategorie sind Technologien zur Beeinflussung der globalen Strahlungsbilanz (RM - Radiation Management), welche z.B. versuchen, mehr Sonneneinstrahlung ins Weltall zu reflektieren. Diskutiert werden beispielsweise die "Injektion“ von Schwefelpartikeln in die Stratosphäre, um Wolkenbildung anzuregen; die Ausbringung reflektierender Materialien zwischen Erde und Sonne; oder die großflächige Aufhellung der Erdoberfläche etwa durch weiße Folien (Caviezel/Revermann 2014).

Die einzelnen Technologien unterscheiden sich in ihrer vielschichtigen technischen, wirtschaftlichen, geografischen und soziopolitischen Auswirkung auf Mensch und Umwelt voneinander und bergen teils erhebliche Risiken (Klepper/Dovern et al. 2016). Großtechnische Eingriffe in das Klima zur Manipulation des Erdsystems nehmen das Risiko von Fehlplanungen und gigantischen öffentlichen Investitionen in Kauf und können nach ihrem Einsatz schwer oder gar nicht kontrolliert bzw. rückgängig gemacht werden, was dramatische Folgen für zukünftige Generationen haben könnte. Abhängigkeit entsteht (Lock-in-Effekt): die Technologie kann nicht "abgeschaltet“ werden und bei einem Zusammenbruch kann es zu sich selbst potenzierenden Klimaeffekten kommen (Termination Shock, siehe IPCC 2018). Der Umsetzungsstand solcher Geoengineering-Technologien, die Auswirkungen von Treibhausgasemissionen nachträglich behandeln wollen, reicht von rein theoretischen Konzepten über Prototypen bis zu bereits lokal eingesetzten Technologien. Derzeit sind weltweit mehr als 800 Projekte zu Wettermodifikation, Strahlungsmanagement oder Treibhausgasabscheidung und -speicherung dokumen- 
tiert, was vermutlich nur ein Teil der tatsächlichen Anzahl ist. ${ }^{1}$ Derzeit finden in Österreich keine staatlich unterstützten klimaverändernden Geoengineering-Aktivitäten statt (BMLFUW 2017). Kleinräumige Wettermodifikation, wie etwa die wissenschaftlich in ihrer Wirkung nicht belegte (Wu et al. 2018) Hagelabwehr mit einem mit Silberjodid-Aceton-Gemisch wird regelmäßig angewendet. ForscherInnen melden sich in regelmäßigen Abständen zu Wort und warnen davor, sich zu sehr auf „Plan B“ zu verlassen, um den Klimawandel zu adressieren, denn die Geoengineering-Maßnahmen können eine Dekarbonisierung des wirtschaftlichen und gesellschaftlichen Alltags nicht ersetzen (Pierrehumbert 2019).

Globale Governance von Geoengineering beruht auf einem generellen Moratorium auf Basis der UN-Konvention über die biologische Vielfalt (kleinere Experimente sind erlaubt), sowie einem späteren rechtsverbindlichen Rahmen für marines Geoengineering (London Protocol). Eine Erweiterung dieses Rahmens wäre notwendig, scheiterte aber bisher. ${ }^{2}$ Es besteht die Gefahr, dass einzelne Länder hier - mit globalen Konsequenzen - vorstoßen (IPCC 2018). Fraglich ist, ob es zu einer unregulierten Anwendung und auch zu einer Kommerzialisierung von GeoengineeringTechnologien kommt und welche Folgen das hätte. Auch eine Militarisierung und das Entstehen von Monopolstellungen in der Klimabeeinflussung sind durch solche Großprojekte denkbar. Diese könnten als politisches Druckmittel eingesetzt werden. Der nationale (Nicht-)Handlungsrahmen sollte hier vorausschauend abgesteckt und gesetzlich verankert werden, damit österreichische EntscheidungsträgerInnen gut vorbereitet auf die internationale Debatte reagieren können. Zusätzlich können kurzund langfristige umweltrelevante, wirtschaftliche und soziale Auswirkungen von den massiven Eingriffen solcher Technologien frühzeitig für die Bevölkerung dargestellt werden.

\section{Zitierte Quellen}

BMLFUW (2017). Nationalrat - XXV. GP Beantwortungen "Geoengineering" (13073/AB), Republik Österreich, Parlament.

Caviezel, C. und C. Revermann (2014). Climate Engineering - Abschlussbericht zum TA-Projekt »Geoengineering «. TAB-Arbeitsbericht 159.

Gernot Klepper et al. (2016). Herausforderung Climate Engineering Bewertung neuer Optionen für den Klimaschutz. Kieler Beiträge zur Wirtschaftspolitik, Institut für Weltwirtschaft Kiel. 8.

IPCC (2018). Global Warming of $1.5^{\circ} \mathrm{C}$ - Special Report 15, Chapter 4: Strengthening and implementing the global response, ipcc.ch/report/sr15/.

Pierrehumbert, R., 2019, There is no Plan B for dealing with the climate crisis, Bulletin of the Atomic Scientists 75(5), 215-221 doi.org/10.1080/00963402.2019.1654255.

1 Die Heinrich-Böll-Stiftung und ETC-Group erstellten 2018 eine Landkarte weltweiter Geoengineering-Aktivitäten, siehe boell.de/en/geoengineering.

2 umweltbundesamt.de/themen/nachhaltigkeit-strategieninternationales/umweltrecht/umweltvoelkerrecht/geoengineeringgovernance\#wirksamer-klimaschutz-oder-grossenwahn. 
Wu, X. et al. (2018). Advances in the evaluation of cloud seeding: Statistical evidence for the enhancement of precipitation. Earth and Space Science, 5, 425-439. DOI:10.1029/2018EA000424. 


\section{G - Gestaltungsoffenheit der Anwendungen für den neuen Mobilfunkstandard nutzen}

\section{Zusammenfassung}

Die Mobilfunktechnologie der fünften Generation (5G) wird im Vergleich zu heutigen Mobilfunktechnologien sehr viel höhere mobile Übertragungsraten ermöglichen. Der neue Standard ist vor allem für die Industrie und das Internet der Dinge (IoT) von hoher Bedeutung. 5G bietet eine hohe Flexibilität und kann genau auf bestimmte Anforderungen zugeschnitten sein: In Fertigungshallen werden Maschinen miteinander verbunden, hohe Bandbreiten werden für die Wiedergabe hochauflösender Videos zur Verfügung gestellt und auf Straßen soll ein besonders schnelles und zuverlässiges Netz mit besonders kurzen Verzögerungszeiten (Latenz) ermöglichen, autonome Fahrzeuge zu steuern. In der Öffentlichkeit wird 5G auch kontrovers diskutiert, wobei es erneut um mögliche gesundheitliche Strahlungsrisiken geht. Für 5G-Anwendungen sind vielfältige Entwicklungsoptionen denkbar und offen, so dass aktuell ein hohes Gestaltungspotential dieser zentralen Infrastruktur vorhanden ist.

\section{Überblick zum Thema}

Mit 5G können die hohen und zugleich sehr unterschiedlichen Anforderungen zukünftiger Anwendungen in den Bereichen Industrie 4.0, Internet der Dinge (IoT), Automatisierung, sowie Logistik 4.0 erfüllt werden. Um dies zu erreichen, ist $5 \mathrm{G}$ darauf ausgerichtet, Latenzzeiten von wenigen Millisekunden zu erzielen, Datenraten von bis zu 20 Gigabits pro Sekunde möglich zu machen und dabei zugleich eine hohe Zuverlässigkeit der Netze sowie eine hohe Positionierungsgenauigkeit zu bieten. Der stets identifizierbare Standort von Objekten über vernetzte Sensorik (siehe auch Thema "Sensorrevolution") stellt ein wesentliches Merkmal neuer Geschäftsmodelle und Dienstleistungen dar, so dass die Positionierung ein integraler Bestandteil des Systemdesigns von 5G-Mobilfunknetzen ist.

Hohe Erwartungen werden auch daran geknüpft, dass der neue Mobilfunkstandard den Alltag verändern wird, z.B. durch Anwendungen in den Bereichen Smart City und Smart Building. Die 5G-Positionierungs-Architektur, die auf der Integration und Vernetzung von allgegenwärtigen Sensoren basiert, bietet eine hohe Leistungsfähigkeit für die Lokalisierung, die neue Möglichkeiten in der Netzarchitektur eröffnet. Die 5G-Lokalisierung ist im Moment primär zur Beschleunigung industrieller und logistischer Prozesse gedacht; Dienstleistungen für den Alltag im Zusammenhang mit der stetig steigenden Zahl von mobilen Sensoren sind noch weniger diskutiert. Da es mit $5 \mathrm{G}$ möglich wird, die Position von Drohnen oder fahrerlosen Transportsystemen sowie das Tracking von Gütern in intralogistischen Prozessen zu realisieren, sind auch Haushalte und Geräte individueller privater NutzerInnen in die Netze integrierbar. Zukunftsanwen- 
öffentliche Diskussion

über Risiken

österreichische

5G-Strategie dungen sind auch für eine bessere Gesundheitsversorgung dank schneller Datenübertragung denkbar, z.B. im Fall, dass Ärzte und Ärztinnen künftig umfangreiche Daten und Videoübertragung bekommen, während der Krankenwagen noch auf dem Weg zur Notaufnahme sind und damit auch steuernd eingreifen können. ${ }^{1}$

Auf der anderen Seite werden in der Öffentlichkeit zunehmend Risiken diskutiert und es gibt Petitionen gegen den 5G-Netzausbau. ${ }^{2}$ Studien öffentlicher Institutionen betonen hingegen die Unbedenklichkeit von 5G, ${ }^{3}$ erkennen aber noch bestehende Ungewissheit an. Doch die Polarisierung hinsichtlich der Gesundheitsbedenken scheint trotzdem zuzunehmen. ${ }^{4}$

Die österreichische 5G-Strategie sieht vor, bis Ende 2023 eine Nutzbarkeit von 5G-Diensten auf den Hauptverkehrsverbindungen möglich zu machen und bis Ende 2025 das Ziel einer nahezu flächendeckenden Verfügbarkeit von $5 \mathrm{G}$ zu verwirklichen. ${ }^{5}$

Auf der Grundlage der Mitteilung der Europäischen Kommission „5G für Europa: ein Aktionsplan" 6 vom September 2016 wurden die Mitgliedsstaaten eingeladen, nationalstaatliche 5G-Strategien als Bestandteil der nationalen Breitband-Pläne auszuarbeiten. In Österreich wurden unter Einbeziehung von Stakeholdern zentrale Probleme identifiziert, Maßnahmen erarbeitet und priorisierte Umsetzungsempfehlungen entwickelt und der Bundesregierung zur geplanten Umsetzung empfohlen. ${ }^{7}$ Im März 2019 hat Österreich als eines der ersten europäischen Länder Frequenzen für

1 Siehe zu diesem Beispiel: handelsblatt.com/unternehmen/it-medien/netzausbaufuer-ericsson-und-nokia-ist-es-besonders-schwer-vom-5g-boom-zuprofitieren/24071874.html. Bei solchen Szenarien bleibt allerdings offen, ob die Technologie zu mehr Versorgungssicherheit führen kann, indem über die digitale Technologie Ärzte und Ärztinnen zur Verfügung stehen, die sonst nicht zur Verfügung stehen, oder ob es zu einem Abbau von Personal im Krankenwagen führt.

2 weact.campact.de/petitions/petition-gegen-5g-netzausbau-in-osterreich; vgl.: derstandard.at/2000100738589/Angst-vor-Strahlung-Petition-gegen-5GNetzausbau, krone.at/1896976.

3 In Österreich z.B.:

bmk.gv.at/themen/telekommunikation/breitband/publikationen $/ 5 \mathrm{~g} / 5 \mathrm{gfaktencheck}$. html; vgl. Mandl, et al. (2018). In Deutschland:

bfs.de/DE/themen/emf/kompetenzzentrum/mobilfunk/basiswissen $/ 5 \mathrm{~g} . \mathrm{html}$. Einen umfassenden Überblick dazu geben Kastenhofer et al. 2020.

4 Es gibt sehr wenige wissenschaftliche Beiträge zum Thema der möglichen Strahlenbelastungen von 5G, insbesondere im Millimeterbereich. Von manchen wird das Vorsorgeprinzip ins Spiel gebracht (und tatsächlich gibt es bereits Moratorien, etwa in Brüssel und in manchen Schweizer Kantonen); damit verbunden ist die Forderung nach Studien, die direkt und spezifisch die gesundheitlichen Auswirkungen von 5G untersuchen (z.B. Simkó und Mattsson 2019, Kastenhofer et al. 2020).

5 Strategie vom April 2018: bmk.gv.at/dam/jcr:20b1f1c7-8557-4fde-9b8d4f75fb8cf291/5Gstrategie_ua.pdf.

6 ec.europa.eu/digital-single-market/en/5g-europe-action-plan.

7 Strategie vom April 2018: bmk.gv.at/dam/jcr:20b1f1c7-8557-4fde-9b8d4f75fb8cf291/5Gstrategie_ua.pdf. 
5G-Mobilfunk versteigert. ${ }^{8}$ Dabei gab es unerwartete Bieterlnnen, neben den überregionalen Handynetzbetreibern kamen auch Anbieter zum Zug, die regionale Netze aufbauen wollen, wie z.B. die Salzburg AG und die Graz Holding. Mit einer städtischen bzw. regionalen und öffentlichen Kontrolle und Eigenständigkeit hinsichtlich des Netzaufbaus ergibt sich die Möglichkeit, regional 5G-bezogene Innovationaktivitäten zu starten, die lokale Akteure in die Entwicklung von Anwendungen und Dienstleistungen früh einbezieht. Denn aufgrund der vielfältigen Anwendungsmöglichkeiten und den offenen Entwicklungsperspektiven bietet $5 \mathrm{G}$ große Chancen für neue Dienstleistungen.

Um potentiellen NutzerInnen ansprechende und reibungslos funktionierende Anwendungen zu bieten, stehen die beteiligten Akteure noch vor einer Vielzahl von Herausforderungen, da noch nicht klar ist, wie die Technologie implementiert wird und welche Vorteile sie Akteuren in Österreich bringen wird. Geräte müssen in die Lage versetzt werden, nach den Spezifikationen der Normen zu arbeiten und die Anbieter von Anwendungen müssen sicherstellen, dass Netzwerkgeräte interoperabel sind, während sie unter hohem Wettbewerbsdruck stehen.

\section{Relevanz des Themas für das Parlament und für Österreich}

Da die Infrastrukturentwicklung in den Grundlagen bereits beschlossen ist und mittelfristig realisiert wird - auch wenn weitere Ausbaustufen wie z.B. die Versteigerung weiterer Frequenzen aktuell wegen der Covid-19-Krise ausgesetzt wurden ${ }^{9}$ - stellen sich weiterhin zwei Fragen: Zum einen sollte der Wissensstand zu den potenziellen gesundheitlichen Risiken (Kastenhofer et al. 2020) in weiterer Folge berücksichtigt werden. Zum anderen stellt sich die Frage, wie der langfristige Nutzen über eine breite 5GInnovationskultur in Österreich gesichert werden kann. Das Thema Gesundheit ist in doppelter Hinsicht von Relevanz, da 5G für eine Vielzahl an Gesundheitsanwendungen nutzbar ist, wie z.B. für Telemedizin und das Monitoring der Vitalwerte bei chronisch Kranken durch Wearables (de Mattos/Gondim 2016).

VerbraucherInnen sollen mit $5 G$ nicht nur ein schnelleres Internet bekommen; vielmehr ermöglicht es $5 G$ auch, dass jedes Gerät, das einen Chip enthält, ständig mit dem Netzwerk verbunden ist: Smartphone, Tablett, Auto, Ampel, Parkuhr gehören genauso dazu, wie Fernseher, Energiemanagement, Haus-Sicherheitssysteme und Kühlschränke. Mit der allgegenwärtigen Vernetzung (siehe auch Thema „Cybersicherheit“) sind damit auch in verschärfter Form Fragen der Privatsphäre, aber auch der Resilienz von digital vernetzen Systemen (z.B. beim autonomen Fahren) zu adressieren.

8 Dabei erhielten sieben Bieter Nutzungsrechte im Band 3,4-3,8 GHz zum Marktwert von insgesamt rund 188 Mio. Euro. Band 3,4 bis $3,8 \mathrm{GHz}$ sind mit geringeren Ausbreitungseigenschaften und hohe Bandbreiten insbesondere für die regionale Versorgung wichtig. Vgl. rtr.at/de/tk/5G-Auction.

9 wienerzeitung.at/nachrichten/digital/digital-news/2056114-Zweite-5G-Auktionwird-wegen-Coronavirus-verschoben.html.
Chancen für neue

Dienstleistungen 
TAB-Studie zu

Gesundheitsrisiken im

Laufen, österreichische Studie liegt vor

Suche nach gesellschaftlichen

Nutzungs- und Wertschöpfungspotentialen in Österreich

\section{Vorschlag weiteres Vorgehen}

Im Rahmen einer Langstudie wäre es sinnvoll, systematisch die langfristigen technologischen Entwicklungsoptionen in zentralen 5G-Anwendungsfeldern zu identifizieren. Da in der Öffentlichkeit zunehmend Risiken thematisiert werden und es Forderungen nach Technikfolgenabschätzung gibt, wird international bereits an Zusammenfassungen des aktuellen Wissensstandes zu gesundheitlichen Risiken elektromagnetischer Felder im Allgemeinen und $5 \mathrm{G}$ im Besonderen gearbeitet ${ }^{10}$ und eruiert, welchen Bedarf es an weiterer Risikoabschätzung gibt. Der Nationalrat hat diesbezüglich 2019 eine erste Überblicksstudie in Auftrag gegeben, deren Endergebnisse seit Februar 2020 vorliegen (Kastenhofer et al. 2020).

Wichtig wäre in weiterer Folge die Analyse, ob in den Architekturen und Anwendungen die Bedenken aufgegriffen werden können, z.B. eine individuelle Kontrolle der aktuellen Exposition möglich ist. Zentral ist es, partizipativ die Infrastrukturoptionen und Innovationspfade in den Anwendungen von 5G zu analysieren, um zu antizipieren, wo hohe gesellschaftliche Nutzungs- und Wertschöpfungspotentiale festzustellen sind und wie zukünftige Anwendungen für neue Branchen nutzbar gemacht werden können und damit in der Breite eine 5G-Innovationskultur gefördert werden kann. Gerade in der gegenwärtig intensiv geführten Gesundheitsdebatte wäre es wichtig den möglichen Beitrag von innovativen 5G Anwendungen zur Etablierung hoher Standards im Gesundheitsbereich auszuloten, ${ }^{11}$ der langfristig zur Stärkung des Vertrauens in das öffentliche Gesundheitswesen beitragen könnte. Zudem wäre festzustellen, ob der internationale und vor allem der europäische Rechtsrahmen ausreichen, um die Frage von 5G-Überwachungspotentialen und Demokratie ausreichend zu adressieren. Dazu gehören auch Fragen privatsphärenfreundlicher Prinzipen in Netzwerkarchitekturen sowie entsprechende Produkt- und Serviceentwicklung.

\section{Zitierte Literatur}

Cisotto, G., Casarin, E. und Tomasin, S., 2020, Requirements and Enablers of Advanced Healthcare Services over Future Cellular Systems, IEEE Communications Magazine 58(3), 76-81.

de Mattos, W. D. und Gondim, P. R. L., 2016, M-Health Solutions Using 5G Networks and M2M Communications, It Professional 18(3), 24-29.

Kastenhofer, K., Mesbahi, Z., Schaber, F. und Nentwich, M., 2020, 5GMobilfunk und Gesundheit; Endbericht, im Auftrag des Österreichischen Parlaments, Nr. ITA-AIT-11, Wien: Institut für Technikfolgen-Abschätzung (ITA) und AIT Austrian Institute of Technology, epub.oeaw.ac.at/ita/ita-projektberichte/ITA-AIT-11.pdf.

\footnotetext{
${ }^{10}$ Eine entsprechende Studie läuft derzeit bis Jahresmitte 2020 am Büro für Technikfolgen-Abschätzung beim Deutschen Bundestag (TAB), siehe tab-beimbundestag.de/de/untersuchungen/u30300.html.

${ }^{11} \mathrm{Zu}$ den Anforderungen und Voraussetzungen für fortgeschrittene Gesundheitsdienstleistungen durch $5 \mathrm{G}$ siehe Cisotto, et al. (2020).
} 
Mandl, P., Pezzei, P. und Leitgeb, E., 2018, Selected Health and Law Issues regarding Mobile Communications with Respect to 5G; in 2018 International Conference on Broadband Communications for Next Generation Networks and Multimedia Applications, hg. v. Plank, T., New York: IEEE.

Simkó, M. und Mattsson, M.-O., 2019, 5G Wireless Communication and Health Effects-A Pragmatic Review Based on Available Studies Regarding 6 to $100 \mathrm{GHz}$, Environmental Research and Public Health 16(18), 3406 


\section{Cloud Computing als politische Herausforderung}

Vielen ist "die Cloud“ ein Begriff, fast alle verwenden ihn. Die größten Anbieter kommen aus den USA, ${ }^{1}$ Europa hinkt trotz einiger Bemühungen nach wie vor hinterher. Unter Cloud Computing versteht man Dienste, um Daten zu speichern und auf diese über das Internet zugreifen zu können. Cloud Services sind aber auch gemietete Software und Rechenleistung aus unterschiedlichen Rechenzentren eines Betreibers. Für die Nutzerlnnen ist dabei oft völlig unklar, wo genau ihre Daten tatsächlich gespeichert sind bzw. woher sie ihre Software beziehen.

Cloud Computing lässt sich als spezielle Form des IT-Outsourcings begreifen. Dementsprechend ergeben sich ähnliche Probleme, insbesondere was die Abhängigkeit von Anbietern betrifft. Verstärkt wird dies noch durch die Intransparenz für die NutzerInnen. Cloud Computing bietet zwar mehr Flexibilität und Datenmobilität, es ist aber im Grunde eine virtuelle Form der Zentralisierung. Damit ergeben sich noch mehr Abhängigkeit und Gefahren für Ausfälle - also weniger Ausfallsicherheit. ${ }^{2}$ Die Resilienz eines Unternehmens ist somit von der Resilienz des Cloud-Betreibers abhängig.

Insbesondere für staatliche Stellen und gesellschaftlich relevante Institutionen und Unternehmen können Cloud Services als kritische Infrastrukturen begriffen werden. Wie kann im Sinne der digitalen Souveränität der Akteure (siehe auch Thema "Digitale Souveränität") ein möglichst minimiertes Risiko erzielt werden? Sind die Nutzungsbedingungen und vor allem die Diversifizierung des Dienstleister-Portfolios hinreichend?

Im globalen Wettbewerb sind insbesondere Microsoft, Amazon und IBM mit etwa einem Drittel Marktanteil relevante Akteure, ${ }^{3}$ die aus europäischer Sicht kritischer Beurteilung bedürfen. Dies umso mehr, als in Zeiten der Covid-19-Pandemie deutlich wurde, dass an vielen öffentlichen und privaten Stellen US-amerikanische Cloud Services verstärkt genutzt werden und damit weitere Abhängigkeiten geschaffen werden. Eine besondere Bedeutung haben dabei die Themen Datenhoheit, Schutz der Privatsphäre und die Anfälligkeit für Missbrauch. Eine steigende Anzahl an Datenverlusten verdeutlicht dementsprechende Gefahren für die Privatsphäre des Einzelnen genauso wie für sensible Geschäftsdaten und die Funktionsfähigkeit wirtschaftlicher und gesellschaftlicher Prozesse

\footnotetext{
1 de.statista.com/statistik/daten/studie/150979/umfrage/marktanteile-derfuehrenden-unternehmen-im-bereich-cloud-computing/.

2 heise.de/newsticker/meldung/Blitzschlag-in-den-USA-stoert-Azure-ActiveDirectory-4155496.html; wired.de/collection/tech/amazon-cloud-ausfall-einsimpler-tippfehler-war-schuld.

3 Amazon bekommt auch das Cloud-Monopol für das US-Militär, siehe fm4.orf.at/stories/2942129/.
} 
(Leimbach et al. 2014). ${ }^{4}$ Mit der Datenschutzgrundverordnung verfügt die EU nun über einen mehr oder weniger vereinheitlichten Rechtsrahmen, was die Entstehung europäischer Anbieter befördern könnte. Das deutlich bessere Datenschutzniveau in der EU kann auch global als positiver Wettbewerbsfaktor genutzt werden. Dementsprechend wurde die European Open Science Cloud (EOSC)-Initiative ${ }^{5}$ ins Leben gerufen. In ihrem Arbeitsplan für 2019/20206 wird die Inbetriebnahme für Ende 2020 angekündigt. Auch in der im Februar 2020 vorgestellten Datenstrategie der Europäischen Kommission wird Cloud-Lösungen eine hohe Priorität zugewiesen. ${ }^{7}$

Darüber hinaus besteht Bedarf an offenen, und damit überprüfbaren Sicherheitsstandards und Cloud-Architekturen (siehe Thema „Dezentralisierte Kollaborationsformen“), sodass sichergestellt werden kann, dass keine Hintertüren oder sog. „lawful interception“-Schnittstellen zum legalen Abhören eingeplant sind, die die Sicherheit von Cloud Services massiv gefährden. Mögliche Regulierungen zum besonderen Schutz und dem Verbot der Weitergabe von Cloud-Daten an Dritte könnten angedacht werden. Insgesamt könnten die Rahmenbedingungen und mögliche Erfolgsfaktoren für eine erfolgreiche europäische/österreichische Initiative ausgelotet werden.

\section{Zitierte Quellen}

Leimbach, T. et al. (2014) Potential and Impacts of Cloud Computing Services and Social Network Websites - Study IP/A/STOA/FWC/2008096/Lot4/C1/SC8; Science and Technology Options Assessment European Parliamentary Research Service: Brussels, europarl.europa.eu/RegData/etudes/etudes/join/2014/513546/IPOLJOIN_ET(2014)513546_EN.pdf.

\footnotetext{
4 Siehe auch kaspersky.com/about/press-releases/2018_costly-cloud-breaches; blog.storagecraft.com/7-infamous-cloud-security-breaches/; forbes.com/sites/louiscolumbus/2018/07/27/ibms-2018-data-breach-studyshows-why-were-in-a-zero-trust-world-now/\#.

5 eosc-portal.eu.

6 op.europa.eu/en/publication-detail/-/publication/3c379ccc-ee2c-11e9-a32c01aa75ed71a1.

7 ec.europa.eu/info/sites/info/files/communication-european-strategy-data19feb2020_de.pdf.
} 


\section{Autonomer öffentlicher Verkehr}

\section{Zusammenfassung}

Der Öffentliche Personen-Nahverkehr (ÖPNV) wird sowohl von schienengebundenen Systemen (U-Bahn, Straßenbahn, Zug) als auch von auf Straßen betriebenen Bussen und Sammeltaxis bedient. Aufgrund der zunehmenden Verfügbarkeit der Technologie und erwarteten Effizienzgewinnen, gibt es international und in Österreich zahlreiche Initiativen, den ÖPNV (teilweise) zu automatisieren. Dabei ist der automatisierte schienengebundene ÖPNV mit 55 U-Bahn Linien in 37 Städten weltweit derzeit wesentlich weiter entwickelt. Straßengebundene autonome Fahrzeuge werden vor allem in Pilotprojekten in Randbereichen großer Städte und auf wenig genutzten Kurzstrecken getestet. Die Erwartungen sind vor allem, dass autonome Systeme im öffentlichen Verkehr eine Brücke zwischen Individualverkehr und ÖPNV bilden sollen. Bei autonomen Systemen stellt sich jedoch eine Reihe von grundsätzlichen Fragen nach Sicherheit, Verantwortung, Maschinenethik und Akzeptabilität sowie nach Art und Kosten der Infrastruktur und den Auswirkungen auf die zuliefernde Industrie und die Arbeitsplätze im Mobilitätssektor. Für eine zukunftsorientierte Befassung und proaktive Gestaltung kann das Parlament ein zentraler Ort sein.

\section{Überblick zum Thema}

Wenn man vom autonomen Fahren spricht, wird meist an selbstfahrende Autos und den autonomen Individualverkehr gedacht. Allerdings können durch die autonome Steuerung von Fahrzeugen auch effizienzsteigernde Potentiale für den öffentlichen Verkehr erzielt werden. Von den Befürworterlnnen werden vor allem Personaleinsparungen und eine möglicherweise höhere Sicherheit ins Treffen geführt. Derzeit werden autonome Systeme vor allem im schienengebundenen ÖPNV breiter eingesetzt. Eine großflächige Anwendung im straßengebundenen Bereich ist derzeit nur auf Pilotanwendungen beschränkt. Dort ist die Automatisierung bereits weiter fortgeschritten. In vielen Städten verkehren seit Jahren fahrerlose U-Bahn Garnituren im Linienverkehr. Im Juli 2016 gab es 55 vollautomatische U-Bahn-Linien in 37 Städten auf der ganzen Welt, die insgesamt 803 km befuhren. Das entspricht gegenüber 2014 einem Anstieg von $14,2 \%$. Es wird prognostiziert, dass bis $20252.300 \mathrm{~km}$ automatisierte Metrolinien in Betrieb sein werden. ${ }^{1}$

Auch Züge im Überlandbereich könnten in Zukunft fahrerlos unterwegs sein. Erste Tests mit Güterzügen gibt es seit 2018 zwischen Rotterdam und Deutschland. ${ }^{2}$ Die bislang entwickelten, autonom fahrenden Güterzüge erreichen das Autonomielevel 3 bis 4. Der Zug fährt zwar computerge-
Effizienzgewinne vs.

Sicherheit, Verantwortung, Ethik und Akzeptabilität

Vorreiter: schienengebundener ÖPNV

\footnotetext{
1 uitp.org/world-report-metro-automation.

2 mobilitymag.de/autonome-zuege-lokfuehrer/.
} 
erste Versuche mit autonomen Bussen

autonome Sammeltaxis steuert, der/die Fahrerln ist aber noch mit an Bord und kann notfalls die Steuerung übernehmen. ${ }^{3}$ Durch die klar abgegrenzte und direkt kontrollier- und beeinflussbare Systemumgebung können die schienengebundenen Systeme wesentlich leichter einen höheren Grad der Automation ${ }^{4}$ erreichen als solche im Straßenverkehr.

Aber auch eigene Busspuren in den Städten und der Einsatz in Außenbezirken sowie im ländlichen Raum bieten etwas einfachere Rahmenbedingungen für den Einsatz autonomer Fahrzeuge im ÖPNV. Auch in Österreich gibt es bereits Versuche mit autonomen Bussen: Im Oktober 2016 wurde in Salzburg ein Testbetrieb mit Minibussen vorgestellt, ${ }^{5}$ der mittlerweile auch erstmals fahrerlos getestet wurde. ${ }^{6}$ In der Salzburger Gemeinde Koppl wurde ein selbstfahrender Kleinbus getestet; dieser verbindet den Ort mit der eineinhalb Kilometer entfernten Haltestelle des BusLinienverkehrs. ${ }^{7}$ Im Jahre 2017 wurde im Rahmen des vom Land Kärnten finanzierten Projektes SURAAA (Smart Urban Region Austria Alps Adriatic) ein autonomer Bus in Pörtschach präsentiert. Dieser ist seit Juni 2018 auch im Regelbetrieb auf einer 1,5 Kilometer langen Strecke im Einsatz. ${ }^{8}$ Seit Frühling 2018 forscht das Projektkonsortium „auto.Bus - Seestadt“ unter Gesamtleitung der Wiener Linien und wissenschaftlicher Leitung des AIT Austrian Institute of Technology an den zwei selbstfahrenden EBussen in der Busgarage Leopoldau: Das vom BMVIT im Rahmen des Programms „Mobilität der Zukunft“ zur Hälfte geförderte Projekt sieht einen regelmäßigen Fahrgasttestbetrieb mit fixer Linienführung und Haltestellen ab dem Frühjahr 2019 in der Seestadt Aspern vor. ${ }^{9}$ Bisher wurden etwa 4000 km gefahren und 4500 Personen befördert, Projektende ist Sommer 2020. ${ }^{10}$

Auch international ${ }^{11}$ zeigt sich, dass vorerst gering genutzte Kurzstrecken an der Peripherie, also die „letzte Meile“ als Zubringer zum Zug und anderen öffentlichen Verkehrsmitteln abgedeckt werden sollen. ${ }^{12}$ In Singapur werden autonome (Sammel-)Taxis als Zubringer zum ÖPNV getestet. Die

3 alstom.com/press-releases-news/2018/12/alstom-succeed-ato-testsbetuweroute.

4 uitp.org/sites/default/files/Metro\%20automation\%20$\% 20$ facts $\% 20$ and $\% 20$ figures.pdf.

5 salzburgresearch.at/presseaussendung/erster-selbstfahrender-bus-in-salzburgautonomerminibus/.

6 salzburgresearch.at/presseaussendung/der-selbstfahrende-digibus-faehrterstmals-fahrerlos/.

7 vcoe.at/news/details/vcoe-durch-automatisierung-nehmen-auto-und-lkwverkehr-stark-zu.

8 kleinezeitung.at/wirtschaft/5447868/Woerthersee_Warum-jetzt-ein-smarterAutobus-durch-Poertschach-faehrt.

9 wienerlinien.at/eportal3/ep/bvContentView.do?contentTypeld=1001\&contentld= 4201783\&programld=4400504\&channelld=-46652.

10 wienerlinien.at/eportal3/ep/contentView.do?pageTypeld=66526\&channelld=47186\&programld=74577\&contentld=5000562\&contentTypeld=1001.

${ }^{11}$ srf.ch/news/schweiz/oeffentlicher-verkehr-revolution-der-selbstfahrenden-busse.

12 elib.dlr.de/103519/1/Individuell_abrufbare_Personentransportsysteme.pdf. 
Verantwortlichen der stark unter Staus leidenden Großstadt möchten herausfinden, ob damit mehr Staus vermieden werden können oder vielleicht das Gegenteil passiert, weil das Angebot so attraktiv ist, dass Menschen von anderen öffentlichen Verkehrsmitteln auf die autonomen Fahrzeuge umsteigen. ${ }^{13}$ Autonome Busse mit Sammeltaxi-Charakter nutzen einen weiteren wesentlichen Vorteil fahrerloser Systeme: Sollten die Busse tatsächlich ohne menschlichen Sicherheits-Operateur unterwegs sein dürfen, könnten sie theoretisch 24 Stunden am Tag „On-Demand“ fahren, d.h. sie werden bei Bedarf via Smartphone-App bestellt und könnten auch mehrere Anfragen autonom bedienen. Damit würde das Konzept „Mobility as a Service“ (MaaS) Realität, was zu einer Stärkung des intermodalen Verkehrs führen könnte.

Die Realisierung autonomen Fahrens im Fernverkehr mit größeren Bussen wird ebenso vorbereitet: Daimler hat in Amsterdam mit einem selbstfahrenden Bus eine $20 \mathrm{~km}$ lange Testfahrt absolviert, bei der der Fahrer nur noch Überwachungsfunktion hat. Linienverkehr in eigens ausgewiesenen Busspuren sei das anvisierte Einsatzgebiet der Neuentwicklung. ${ }^{14}$ Die Marktreife ist für 2021 geplant. ${ }^{15}$ Der größte chinesische Hersteller von Bussen, Zhengzhou Yutong Bus Co. Ltd. mit 12 \% Anteil am Weltmarkt, unternimmt Testfahrten mit großen Bussen im regulären Straßenverkehr. Die Firma beschäftigt über 1.000 Angestellte allein in der Entwicklungsabteilung für Busse und gibt an, die Serienreife 2019 zu erreichen. ${ }^{16}$

Für den autonomen Anteil am ÖPNV gelten natürlich ähnliche Herausforderungen und Problemfelder wie beim autonomen Individualverkehr, nicht nur in Hinblick auf den rechtlichen Rahmen (Stichwort: Verantwortlichkeit und Versicherung): Wie müsste die Infrastruktur ausgebaut werden? Welche Technologien müssten noch zur Umsetzung von Fahrzeug-zuInfrastruktur- und Fahrzeug-zu-Fahrzeug-Kommunikation entwickelt und optimiert werden? Wie sind die potenziellen Auswirkungen auf den Arbeitsmarkt (Bus-, Lok-, TaxifahrerInnen vs. EntwicklerInnen, Data Analysts, SystemadministratorInnen etc.) einzuschätzen und welche (Aus-) Bildungsmaßnahmen wären notwendig? Wie kann ein möglichst friktionsfreies Miteinander von autonomen Systemen und traditionellen Verkehrsteilnehmerlnnen in unterschiedlichen Fortbewegungsarten sichergestellt werden? Welche Auswirkungen auf und mögliche Veränderungen im Mobilitätsverhalten wären zu erwarten und wie würden diese auf die Stadtplanung bzw. das Verhältnis von Stadt und Land zurückwirken? Nicht zuletzt ist die Frage zu stellen, inwieweit sich die Einstellung zu Besitz/Eigentum verändert bzw. ob sich Angebote der Sharing-Economy großflächig durchsetzen könnten (Chaloupka et al. 2015)? autonome Fernbusse

viele offene Herausforderungen

\footnotetext{
13 heise.de/-2534980.

14 orf.at/stories/2350006/.

15 heise.de/-3270072

16 ingenieur.de/Branchen/Verkehr-Logistik-Transport/Fahrerloser-Bus-imoeffentlichen-Verkehr-unterwegs.
} 


\section{Gefährdung der}

Privatsphäre

öffentliche Debatte dazu fehlt noch

Einbindung von

Stakeholdern,

ExpertInnen und

BürgerInnen
Der autonome Verkehr würde generell ein beispielloses Datenvolumen produzieren. Insbesondere in einem MaaS-Szenario, bei dem über Apps öffentliche und individuelle Verkehrsdienstleistungen gebucht und abgerechnet werden, würde nachvollziehbar, ab wann die Menschen zur Arbeit gehen, auf welchen Wegen und wohin sie in ihrer Freizeit fahren. Somit bestehen neben dem Optimierungspotential für die Verkehrs- und Stadtplanung vor allem Bedenken bezüglich der Gefährdung der Privatsphäre, die einseitige ökonomische Verwertung dieser Daten und deren möglicher Missbrauch (zu Kfz siehe Krieger-Lamina 2016).

\section{Relevanz des Themas für das Parlament und für Österreich}

Auch in Österreich gibt es bereits zahlreiche Bemühungen, den ÖPNV zu „automatisieren“ - jedoch bislang ohne entsprechende Diskussion, ob dies überhaupt wünschenswert ist und welche Rahmenbedingungen notwendig wären. Zentrale Zukunftsthemen sind Fragen der Regulierung autonomer Systeme (nicht nur im Straßenverkehr). Wie wird Verantwortung verteilt? Wie können Haftungsfragen geregelt werden und welche Herausforderungen ergeben sich bezüglich individueller Nutzungsprofilbildung und Datenschutz? Da sich auch eine Reihe ethischer Fragen auftun, sollte ein breiter gesellschaftlicher Diskurs initiiert werden. Dieser könnte vom Parlament getragen werden. Darüber hinaus sind Fragen der Forschungsförderung und die parlamentarische Weiterentwicklung bestehender Politiken ${ }^{17}$ sowie die Gestaltung von Rahmenbedingungen für die Entwicklung der involvierten Industrien besonders relevant.

\section{Vorschlag weiteres Vorgehen}

Eine Erhebung des Ist-Zustandes in der technischen Entwicklung von Gesamtsystemen und von deren Weiterentwicklungspotenzial bildet die Grundlage für eine tiefergreifende Abschätzung und Bewertung möglicher sozialer, politischer, regulatorischer und wirtschaftlicher Folgen. Auf dieser Basis könnten dann Empfehlungen für Förder- oder Regulierungsmaßnahmen zum gesetzlichen, institutionellen und organisatorischen Umgang mit dem autonomen ÖPNV erarbeitet werden. Vorzugsweise sollte dies unter Einbindung von Stakeholdern und Expertlnnen geschehen. Die Frage der Akzeptabilität könnte darüber hinaus in regional begrenzten Verfahren mit Bürgerlnnen-Beteiligung diskutiert werden. Die Technik entwickelt sich gerade aus dem Labor heraus in die Realität der Anwendungen: ein idealer Zeitpunkt, den wichtige Rahmen für die Nutzung abzustecken.

\footnotetext{
${ }^{17}$ Z.B.

bmk.gv.at/themen/alternative_verkehrskonzepte/automatisiertesFahren.html.
} 


\section{Zentrale weiterführende Quellen}

Chaloupka, C. et al. (2015). Nachhaltige Mobilität aus sozioökonomischer Perspektive. Diskussionspapier. ITA-manu:script. Wien, ITA, epub.oeaw.ac.at/ita/ita-manuscript/ita_15_02.pdf.

Krieger-Lamina, Jaro (2016) Vernetzte Automobile. Datensammeln beim Fahren - von Assistenzsystemen zu autonomen Fahrzeugen.

Endbericht. Bericht-Nr. 2016-02; ITA: Wien; im Auftrag der Bundesarbeitskammer. 


\section{Infrastruktur für Elektromobilität}

Elektro-Kraftfahrzeuge sind eine Option, um Mobilität umweltfreundlicher zu gestalten: Sie weisen einen deutlich höheren Wirkungsgrad auf und verbrauchen im Betrieb wesentlich weniger Energie als Fahrzeuge mit Verbrennungsmotoren. Im Betrieb entstehen weder Treibhaus- noch sonstige gesundheitsgefährdende Abgase (sofern mit erneuerbarer Energie geladen wurde) und auch deutlich weniger Lärm. Immer mehr Staaten und Regionen fördern daher den Umstieg von fossil betriebenen auf elektrische Fahrzeuge und planen, dass benzin- und dieselgetriebene $\mathrm{Kfz}$ bald nicht mehr neu zugelassen werden dürfen: In Großbritannien soll dies bis 2035 geschehen, ${ }^{1}$ in Frankreich bis 2040, und Norwegen will dieses Ziel sogar bis 2025 erreichen. Österreich will bis 2050 einen weitestgehend klimaneutralen Verkehrssektor erreichen und setzt ebenfalls auf EMobilität. ${ }^{2}$

Abgesehen von diversen Fördermaßnahmen für die Fahrzeuge selbst bedarf es dazu des Aufbaus einer entsprechenden Infrastruktur (NEKP 2019, S. 123). Hierbei geht es insbesondere um die Sicherstellung einer ausreichenden Versorgung mit Strom. Ein wichtiger Teil ist dabei der Aufbau eines flächendeckenden Netzes an Ladestationen, sowohl im öffentlichen Raum entlang von Straßen, auf Parkplätzen usw. als auch auf privaten Flächen, insbesondere in Garagen und bei gewerblichen und öffentlichen Fahrzeugflotten. Dieser Ausbau stellt eine technische und finanzielle Herausforderung dar: Die Versorgung einer großen Anzahl von (Schnell-)Ladestationen würde einen Ausbau der Stromleitungskapazitäten benötigen, etwa im Zuge der Um- bzw. Aufrüstung von Tankstellen entlang des hochrangigen Straßennetzes; insofern neue Stromleitungen benötigt werden, wäre auch mit längeren Bewilligungsverfahren zu rechnen. Eine weitere Herausforderung stellt die flächendeckende Ausstattung von Garagen in Mehrparteienhäusern dar (Wohnungseigentumsrecht, Baurecht, Brandschutz). Darüber hinaus werden sich bei zunehmendem Einsatz von EFahrzeugen und entsprechenden Ladestationen auch die Anschlussleistungen für private Haushalte deutlich erhöhen, d.h. dass vor allem die Niederspannungsnetze verstärkt bzw. ausgebaut werden müssten.

Österreich liegt aktuell europaweit im Spitzenfeld in Hinblick auf die Anzahl der E-Ladestationen, wenngleich weiter hinter den Spitzenreitern Norwegen und Niederlande, ${ }^{3}$ wo die Ladestationen allerdings sehr ungleich verteilt sind. In Österreich liegt Vorarlberg voran, wo auch die meisten E-Fahrzeuge zugelassen sind. Es wird weiter investiert, z.B. derzeit in

\footnotetext{
1 zeit.de/wirtschaft/2020-02/grossbritannien-verbrennungsmotoren-verbot-2035.

2 Siehe bmvit.gv.at/verkehr/elektromobilitaet/index.html und aktueller Gesamtverkehrsplan für Österreich: bmvit.gv.at/verkehr/gesamtverkehr/gvp/index.html.

3 EU Transport Scoreboard (letzte verfügbare Zahlen aus 2016) ec.europa.eu/transport/facts-fundings/scoreboard/compare/energy-unioninnovation/ev-charging-points_en.
} 
der Stadt Wien. ${ }^{4}$ Dennoch ist offensichtlich, dass die derzeitige Ladeinfrastruktur für eine stärkere Verbreitung von E-Fahrzeugen nicht ausreichend ist. Effiziente und kundenfreundliche Abrechnungssysteme zwischen den verschiedenen Anbietern („E-Roaming“) ebenso wie Standardisierungen sind ebenfalls wichtige Elemente beim Ausbau der Ladeinfrastruktur. Ein weiteres wichtiges Thema für $F \& E$ und Infrastrukturplanung sind die Rahmenbedingungen für eine bessere Einbindung zusätzlicher Stromerzeugung aus erneuerbaren Energien (Wind und Photovoltaik), etwa bei Park\&Ride-Anlagen mit Solardächern oder auch bei teilautonomen Lösungen im privaten bzw. halböffentlichen Bereich. ${ }^{5}$

Um noch weitergehende Entwicklungen auf dem Weg zur Elektrifizierung des Verkehrs umzusetzen, würde es noch größerer Investitionen in die Infrastruktur bedürfen: In der Nähe der schwedischen Stadt Gävle wird beispielsweise seit 2016 eine „elektrische Straße“ getestet, bei der hybridelektrische LKW an eine Oberleitung andocken können (siehe Thema "Smarte Straßen"). ${ }^{6}$ Ohne Oberleitungen, dafür mit einer aufwändigen Infrastruktur unter der Straßenoberfläche würden Systeme zur kabellosen Stromübertragung auf fahrende Fahrzeuge auskommen, ${ }^{7}$ und werden etwa in Großbritannien in der aktuellen Industriestrategie massiv gefördert. ${ }^{8}$ In Zukunft könnte auch der Flugverkehr zumindest zum Teil elektrifiziert werden, wodurch weitere Investitionen in die Ladeinfrastruktur notwendig würden. ${ }^{9}$

Zusammengefasst geht es bei der Infrastruktur für Elektromobilität um mehrere Themen: die Bereitstellung finanzieller Mittel, um adäquate Rahmensetzung (Recht, Standards, Genehmigungsverfahren), den Schutz der VerbraucherInnen (Preistransparenz), die Vergabe von geeigneten Standorten im öffentlichen Raum und nach wie vor um Fragen der Forschung und Entwicklung (optimale Auslastung der Netze, Integration erneuerbarer Energien).

\section{Zitierte Literatur}

NEKP (2019) Integrierter Nationaler Energie- und Klimaplan für Österreich, Periode 2021-2030 gemäß Verordnung (EU) 2018/1999 über das Governance-System für die Energieunion und den Klimaschutz, Wien, 18.12.2019, bmlrt.gv.at/umwelt/klimaschutz/ klimapolitik_national/nationaler-energie-und-klimaplan.html.

\footnotetext{
4 tanke-wienenergie.at/1000-ladestellen/.

5 Vgl. z.B. Giraffe 2.0, innoventum.se/wpcontent/uploads/Giraffe_leaflet_A4_ENG_160930_150dpi.pdf.

6 scania.com/group/en/home/newsroom/news/2016/worlds-first-electric-roadopens-in-sweden.html.

7 powerelectronics.com/automotive/wireless-charging-electric-vehicles/.

8 gov.uk/government/publications/industrial-strategy-building-a-britain-fit-for-thefuture.

9 electrive.net/2020/02/17/schweden-flughaefen-erhalten-infrastruktur-fuer-eflugverkehr/.
} 


\section{Renaissance des Radverkehrs}

Das Fahrrad entwickelt sich zu einem zentralen Bestandteil des urbanen Verkehrs und einige der fortschrittlichsten Konzepte in Stadtplanung und Mobilität konzentrieren sich auf das Fahrrad - wieder, denn schon in den 1940ern wurden bis zu $85 \%$ aller Wege in vielen europäischen Städten mit dem Rad zurückgelegt (Larsen 2016). Gleichzeitig verbreiten sich auch andere nicht-motorisierte und E-motorisierte, teils spielerische Fortbewegungsmittel und Sportgeräte, wie beispielsweise Tretroller, Longboards, Inlineskates oder elektrische Einräder. Der rechtliche Rahmen für deren Nutzung im Straßenverkehr ist teilweise sehr verschieden, abhängig davon ob sie nach STVO als Fahrzeug oder als Spielzeug gelten, ${ }^{1}$ ein Segway (bis $25 \mathrm{~km} / \mathrm{h}$ ) gilt beispielsweise als Fahrrad ebenso wie ein EScooter; für Hoverboards ist der rechtliche Rahmen unklar. ${ }^{2}$ Das Radfahren verursacht praktisch keine Umweltschäden, fördert die Gesundheit durch körperliche Aktivität, nimmt wenig Platz in Anspruch und ist sowohl bei den direkten Nutzungskosten als auch bei den öffentlichen Infrastrukturkosten wirtschaftlich (Pucher/Buehler 2017). Radfahren ist ökologisch, sozial und wirtschaftlich nachhaltiger als jedes andere Verkehrsmittel, und hat damit klimapolitisch eine entscheidende Rolle (Deffner 2018). Trotz eines erklärten Interesses zur Förderung der aktiven Mobilität ${ }^{3}$ sind die Zielsetzungen mit einer Erhöhung auf 13\% Radverkehrsanteil bis $2025^{4}$ weit entfernt von einer radzentrierten Ausrichtung des urbanen Verkehrs. Vorreiterstädte haben seit den 1970er-Jahren konsequent in Radinfrastruktur und Begleitmaßnahmen investiert und zeigen heute Anteile von $30 \%\left(\right.$ Kopenhagen $\left.^{5}\right)$ oder $48 \%$ (Amsterdam $\left.{ }^{6}\right)$. Als Hinderungsgrund wird beispielsweise in Hinblick auf Wien oft der überdurchschnittlich gute Ausbau der öffentlichen Verkehrsmittel genannt. Dennoch gibt es gerade im urbanen Bereich Maßnahmen, deren Umsetzung den Radverkehr stark fördern würden, beispielsweise die Verkehrsberuhigung von Wohnstraßen oder der Bau und Ausbau von Radschnellwegen. Radschnellwege können den Modalsplit zugunsten des Fahrrads stark erhöhen (Agarwal et al. 2019, Ornetzeder et al. 2016). Der Anschluss des urbanen Umlandes ermöglicht PendlerInnen, mittlere Strecken zurückzulegen und auf das Auto zu verzichten. Physisch abgetrennte und geschützte Wege sind ein zent-

\footnotetext{
1 oeamtc.at/thema/kindersicherheit/kleinfahrzeuge-im-strassenverkehr/.

2 ots.at/presseaussendung/OTS_20160629_OTS0182/e-bikes-segways-undhoverboards-der-rechtsschutzversicherer-arag-klaert-auf.

3 BMGF (2013) Nationaler Aktionsplan Bewegung (NAP.b), bmgf.gv.at/home/Nationaler_Aktionsplan_Bewegung.

4 BMLFUW (2015) Masterplan Radfahren 2015-2025, bmnt.gv.at/dam/jcr:9829acb00928-401a-ae82-3a67aff817fd/43_MP_Radfahren_de.pdf.

5 Copenhagen city cyclists facts figures (2017) cyclingembassy.dk/2017/07/04/copenhagen-city-cyclists-facts-figures-2017/.

6 Netherlands Institute for Transport Policy Analysis (2018) Cycling Facts www.government.nl/binaries/government/documents/reports/2018/04/01/cyclingfacts-2018/Cycling+facts+2018.pdf.
} 
rales Erfolgsrezept für die Förderung des Radverkehrs, weil NutzerInnen effizient und vor allem sicher ans Ziel kommen (Buehler 2016). In innerstädtischen Bereichen zeigen auch Begegnungszonen von Fußgänger-, Rad- und Autoverkehr Vorteile. In vielen chinesischen Städten sind EBikes, mit 250 Millionen registrierten Rädern bereits ein primärer Verkehrsmodus und werden vorrangig zum Pendeln benutzt und nicht wie in Europa für Freizeitaktivitäten (Ma et al. 2019). Es zeigt sich aber auch, dass E-Bikes ein eklatant höheres Unfallrisiko als nicht-motorisierte Räder haben (Ma et al. 2019). Bikes und Sharing-Systeme für Räder, Roller und andere Fortbewegungsmittel werden aller Voraussicht nach in den nächsten Jahren weiter starken Einfluss auf die Mobilitätskultur haben (Pucher/ Buehler 2017). Auch die intermodale Nutzung verschiedener Verkehrsmittel nimmt zu. Erste Studien zeigen, dass AutofahrerInnen eher auf eBikes umsteigen als auf nicht-motorisierte Räder oder öffentlichen Transport (Kroesen 2017) und dass E-Bikes den Gesamtradverkehr erhöhen (Fyhri/Fearnley 2015). Trade-offs zwischen Elektro- und Muskelkraftmobilität sollten näher untersucht werden, wie auch der gesamte Lifecycle von Elektrorädern. Auch sind Nutzungskonflikte bereits heute an der Tagesordnung, diesen kann aber durch verstärkten infrastrukturellen Ausbau und Regelungen begegnet werden. Die wissenschaftliche Erhebung solcher Konflikte, Maßnahmen zum Umgang sowie Ansätze für die Überwindung von Umsetzungshürden kann einer vorausschauenden Politik Empfehlungen liefern, um eine zukunftsfähige Verkehrspolitik zu gestalten.

\section{Zitierte Quellen}

Agarwal, A., Ziemke, D. und Nagel, K., 2019, Bicycle superhighway: An environmentally sustainable policy for urban transport, Transportation Research Part A: Policy and Practice sciencedirect.com/science/article/pii/S096585641731162X.

Buehler, R., Dill, J. (2016). Bikeway networks: A review of effects on cycling. Transport Reviews 36(1): 9-27.

Deffner, J. (2018). Fuß- und Radverkehr. Verkehrspolitik. O. Schwedes. Wiesbaden, Springer VS: 415-444.

Fyhri, A., N. Fearnley (2015). Effects of e-bikes on bicycle use and mode share. Transportation Research D: Transport \& Environment 36: 4552.

Kroesen, M. (2017). "To what extent do e-bikes substitute travel by other modes? Evidence from the Netherlands." Transportation Research D: Transport \& Environment 53: 377-387.

Larsen, J. (2016). The making of a pro-cycling city: Social practices and bicycle mobilities. Environment and Planning A 49(4): 876-892.

Ma, C., Yang, D., Zhou, J., Feng, Z. und Yuan, Q., 2019, Risk Riding Behaviors of Urban E-Bikes: A Literature Review, International Journal of Environmental Research and Public Health 16(13).

Ornetzeder, M. C., Leo; Gutting, Alicia (2016). Monitoring urbaner Technologien, Berichte aus Energie- und Umweltforschung, Nr. 18/2016 bmvit. Wien.

Pucher, J., R. Buehler (2017). Cycling towards a more sustainable transport future. Transport Reviews 37(6): 689-694. 


\section{Inwertsetzung von Natur für industrielle Prozesse: Biosanierung, Bioeffektoren und Biosensorik}

\section{Zusammenfassung}

Biobasierte Prozesse und Materialien sind schon seit längerem ein bedeutendes Thema für die Innovationspolitik. Der zukünftige Trend liegt in der Skalierung der Herstellungsverfahren von biobasierten Innovationen auf industrielle Fertigungsmaßstäbe. Das hohe Potenzial von naturbasierten Lösungen machen aktuelle Entwicklungen im Bereich deutlich; Biosanierung, Bioeffektoren und Biosensorik sind jedoch nur drei Beispiele für dieses umfassende Technologiefeld. Neben grundlegenden Fragen das Natur-Gesellschaftsverhältnis betreffend (z.B. In welchem Ausmaß soll die Natur vom Menschen überhaupt als Ressource betrachtet und ausgebeutet werden? Hat sie intrinsischen Wert, der nicht vollständig quantitativ erfasst werden kann?), streifen biobasierte Industrielösungen weitere Problemfelder, die gesellschaftsrelevante Perspektiven beinhalten (z.B. Einsatz umstrittener Gentechnologien, Transformationshürden in der Politik). Ein transparenter Prozess zur gesellschaftlichen Verhandlung von Werten der Natur wäre für die zukünftige Weiterentwicklung von naturbasierten Lösungen empfehlenswert.

\section{Überblick zum Thema}

Wer denkt, dass Industrie das Gegenteil von Natur ist und lediglich nach deren Ausbeutung trachtet, hat die Entwicklungen der letzten zwanzig Jahre verpasst. Spätestens seit der umstrittenen Studie ${ }^{1}$ zum ökonomischen Wert von Ökosystemdienstleistungen ${ }^{2}$ (TEEB-Studie) ist klar, dass Natur nicht außerhalb von Industrie und Wirtschaft steht, sondern als integraler Bestandteil gesellschaftlicher Systeme und damit als gestaltbar gilt (TEEB 2010). Anhand der ökonomischen Bewertung von Ökosystemdienstleistungen und biologischen Parametern wie Biodiversität, zielte der TEEB-Bericht darauf ab den Wert von Natur für die Sphäre der Wirtschaft greifbar zu machen. Neben diesem grundlegenden Unterfangen werden in der Praxis vermehrt naturbasierte Industrielösungen erforscht und entwickelt, die auf der technischen Nutzbarmachung von natürlichen Systemen und deren Funktionen aufbauen. Dieser Trend wird durch die zunehmende Anerkennung und durch das Bewusstsein, dass die Natur tragfähige Lösungen für ökonomische und soziale Probleme bieten kann,
Ökosystemdienstleistun gen und der ökonomische Wert der Natur

\footnotetext{
1 Das Unterfangen einer quantitativen und monetären Bewertung von „natürlichen" und damit auch ideellen und kulturellen Beständen und Prozessen wurde nicht nur von Verfechterlnnen des Naturschutzes, sondern auch von WissenschaftlerInnen kritisch reflektiert; siehe dazu bspw. Schröter, et al. (2014)

2 Ökosystemdienstleistungen bzw. Ökosystem-Services beschreiben ganz allgemein den Nutzen bzw. die Vorteile, die Menschen von Ökosystemen beziehen. Ein Beispiel dafür wäre die Bestäubung bzw. Verteilung von Pollen oder auch die Filterung und Speicherung von Wasser.
} 
Biosanierung bzw. Bioremediation

Bioeffektoren gestärkt (siehe Industrie 4.0 und Bioökonomie). Auch die Einsicht, dass der Aufbau und Wiederaufbau von natürlichem Kapital einen hohen Aufwand und Investitionen erfordert, treibt die Suche nach „intelligenten“ technischen und natur-basierten Lösungen in der Industrie voran.

Zukunftstechnologiefelder wie Bionik (siehe Biomimikry und Bionik), bioinspirierte und -basierte Materialen (siehe Biobasierte Materialien) und künstliche Photosynthese (siehe Künstliche Photosynthese) sind konkrete Beispiele für naturbasierte Lösungen. Zwei verbindende Elemente der unterschiedlichen Bereiche sind zum einen die innovative Nutzung von natürlichen Ressourcen als Ausgangsbasis für die industrielle Produktion und zum anderen die Ableitung von Funktionsprinzipien sowie die innovative Nutzung von Funktionen der Natur.

Diesen Schritt geht auch die Biosanierung bzw. Bioremediation ${ }^{3}$ : hier wird die Natur selbst zur „Verbesserung“ bzw. „Wiederherstellung“ von Natur genutzt und gilt damit als systemische Ressource für naturbasierte industrielle Prozesse. In der Praxis bedeutet die Reparatur von Natur, dass beispielsweise verschmutzte oder mit Schwermetallen belastete Böden oder Wasserkörper saniert, d.h. durch den Eintrag von Mikroben oder Pflanzen kuriert oder von Schadstoffen gereinigt werden (Ojuederie\&Babalola 2017). Das Prinzip ist nicht neu; seit Jahrzehnten setzt z.B. die stein- und keramische Industrie unter dem Schlagwort „Renaturierung“4 auf eine naturbasierte Lebensraumverbesserung: stillgelegte Abbauflächen (Steinbrüche, Sand- und Kiesgruben) werden sich selbst, d.h. „der Natur“ überlassen und bieten gerade aufgrund der vorher erfolgen radikalen Eingriffe (Sprengungen, Grabungen, u.Ä.) optimale Ausgangsbedingungen für die Entstehung neuer, biodiverser Ökosysteme, die von Natur aus eher selten sind. In Anbetracht zunehmender Zerstörung von natürlichen Ökosystemen, bieten diese industriell geschaffenen Lebensräume gerade für bedrohte und seltene Tier- und Pflanzenarten Zufluchtsorte und bringen nicht nur als beliebte Ausflugsziele gesellschaftlichen Mehrwehrt.

Mit neuen wissenschaftlichen Erkenntnissen bspw. in der Molekularbiologie (siehe Zellfabriken der Zukunft) oder Genetik (siehe Genome editing (CRISPR/Cas9) in der Pflanzenzucht) lässt sich das Funktionsprinzip naturbasierter Lösungen auf andere, industrielle Anwendungsgebiete und sektoren übertragen. Ein Beispiel dafür ist die Forschung an sogenannten Bioeffektoren, d.h. z.B. an speziellen Mikroorganismen, die in Symbiose mit bestimmten anderen Bakterien zur Verbesserung der Nutzpflanzeneigenschaften (v.a. Wachstum) und zur biologischen Kontrolle von Krankheitserregern beitragen (Duque et al. 2019). Als Konsequenz lässt sich die chemische Düngergabe und der Einsatz von chemischen Antimykoti$\mathrm{ka}^{5}$ reduzieren. Um die Mechanismen zu verstehen, wie die Mikroben in die Pflanzenwurzeln gelangen und sich in der gesamten Pflanze ausbrei-

3 Bioremediation bezeichnet den Einsatz von Organismen (Prokaryonten, Pilze oder Pflanzen) zur biologischen Entgiftung von Ökosystemen, die verunreinigt oder mit Schadstoffen belastet sind.

4 forumrohstoffe.at/umwelt/\#nachhaltigkeit.

5 Antimykotika sind antimikrobielle Substanzen, die gegen Erkrankungen wirken, die von Pilzen verursacht werden. 
ten, werden im Vorfeld unter gesicherten Laborbedingungen genetisch veränderte Organismen im Rahmen von in vitro-Versuchen und beim Aufbau von Kontrollsystemen im Gewächshaus eingesetzt.

Ein weiteres Beispiel für naturbasierte, industrielle Lösungen sind Biosensoren, die zukünftig nicht nur für die Erhebung von Umweltparametern, wie Temperatur oder Luftfeuchtigkeit eingesetzt werden könnten (siehe Pflanzen als vernetzte Umweltsensoren), sondern auch in der medizinischen Anwendung hohes Potenzial haben. Gegenwärtig wird in Österreich eine Pilotlinie zur Herstellung papierbasierter elektrochemischer Teststreifen zur quantitativen Biosensorik in Flüssigkeiten aufgebaut ${ }^{6}$. Das Papier dient hierbei als Substrat für die gedruckten Leiterbahnen und für einen Mikrochip sowie die gedruckte Batterie und den Biosensor. Das IMPETUS-Projekt zielt auf die Entwicklung einer schnellen, kostengünstigen und patientennahen Labordiagnostik. Als Anwendungsbeispiel dient hierbei ein Teststreifen, der die Unterscheidung zwischen viraler und bakterieller Infektion ermöglicht, wobei die individuellen Messdaten direkt auf das Smartphone übertragen werden sollen. Herkömmliche Teststreifen ermöglichen qualitativen Analysen (z.B. Ja/Nein-Ergebnisse beim Schwangerschaftstest); die neu-entwickelten Teststreifen erweitern das Analysespektrum um quantitative Verfahren, die anhand einer geringen Menge vom Testmedium präzise quantitative Ergebnisse zu den nachzuweisenden Stoffen (z.B. Antigene) liefern. Das größte Potenzial wird in der Anpassung der Technologie für industrielle Herstellungsprozesse („Rolle-zuRolle-Verfahren“, ähnlich wie im Zeitungsdruck) gesehen. Hier beschleunigen auch neue Technologien, wie bspw. 3D-Druck oder MicrochipTechnologien anhand derer die Teststreifen erzeugt werden, die Entwicklung in Richtung industrielle Verfahren und erhöhen damit die Effizienz von naturbasierten Prozessen.

\section{Relevanz des Themas für das Parlament und für Österreich}

Gerade in Österreich besitzt das Zukunftsthema der naturbasierten Lösungen ein hohes Potenzial: traditionelle Verfahren und Prozesse der Industrie umfassen einerseits Beispiele und Vorbilder für naturbasierte Lösungen; andererseits besteht hohes Innovationspotenzial - wie im vorherigen Abschnitt beschrieben - in der Biosanierung, Biosensorik und beim Einsatz von Bioeffektoren, sowie in den Bereichen Textil und Cellulose, in den Bereichen Bau- und Dämmstoffe aus nachwachsenden Rohstoffen, Papiermaschinen und Zubehör, Polysacharide, Papier, Stärke und bei den biobasierten Spezialprodukten, insbesondere in Hinblick auf ihre Herstellverfahren (Ganglberger et al. 2017). Die Erschließung weiterer Industriesektoren für naturbasierte Lösungen ist vielversprechend, z.B. könnte der Einsatz von papierbasierten, quantitativen Teststreifen im Wein- und Obstbau vereinfachte Analysemöglichkeiten zur Qualitätssicherung bieten. Die Verknüpfung von biobasierten und digitalen Technologien eröffnet weitere Entwicklungspfade, z.B. für neue Verfahren in der weißen, roten und grünen Biotechnologie oder für personalisierte medizinische An-
Biosensorik für

medizinische

Anwendungen
Hohes Potenzial in der

Entwicklung

naturbasierter Lösungen

6 project-impetus.com/. 
Ethische und soziale Aspekte: sozial gerechte

Verteilung des gesellschaftlichen Mehrwerts inwertgesetzter Natur? wendungen (z.B. einfache Blutzuckermessung). Die zukünftige Entwicklung wird auch davon abhängen, ob die Kombination von digitalen und naturbasierten Prozessen zukünftig Fuß fasst und ob es gesellschaftlichen Akzeptanz für eingesetzte, gesellschaftlich umstrittene Techniken (wie z.B. gentechnische Veränderung) geben wird (siehe dazu auch Künstliches Leben).

\section{Vorschlag weiteres Vorgehen}

Naturbasierte Lösungen und Ansätze haben prinzipiell ein hohes Zukunftspotenzial. Gleichzeitig wird bei näherer Betrachtung deutlich, dass ein innovatives Forschungsklima und sichere Testfelder Voraussetzung für die nachhaltige Weiterentwicklung sind. Aus gesellschaftlicher Perspektive stehen nicht nur „heiße Themen“, wie Gentechnologie, im Zusammenhang mit Inwertsetzung von Natur: die wirtschaftliche Nutzung von natürlichen Ressourcen ist mit einer Reihe von ethischen und sozialen Fragestellungen verknüpft. Kritische Einschätzungen sind mit der zentralen Metapher - der wirtschaftlichen Produktivität bzw. Wertigkeit von Ökosystemen - verbunden und verweisen auf ein daraus resultierendes ausbeuterisches Naturverhältnis. Wenn Natur einzig als Ressource und Quelle für Konsum gilt, stellt sich die Frage, welchen Wert die langfristige Erhaltung von einzigartigen Lebensräumen für die Gesellschaft hat. Auch der starke Fokus auf den Erhalt der Biodiversität als Indikator für gesellschaftlichen „Mehrwert“ wirft die Frage auf, wie vermieden werden kann, dass einzelne Aspekte und „Dienstleistungen“ von Ökosystemen zu Ungunsten einer gesamtheitlichen Betrachtungsperspektive in den Vordergrund gestellt werden. Gerade in der Ökologie hat die Systemebene als Analyserahmen hohe Bedeutung und spätestens, wenn man natürliche Lebensräume mit dem Menschen zusammendenkt wird klar, dass die Bewertung von einzelnen Funktionen nur gemeinsam mit den Betroffenen bzw. in den Ökosystemen Lebenden erfolgen kann. So haben bestimmte Ökosysteme für bestimmte Gesellschaftsgruppen mehr oder weniger Bedeutung und auch der unterschiedlich geregelte Zugang zu natürlichen Ressourcen und Ökosystem-Services wirft Fragen zur sozial gerechten Verteilung des erzielten Mehrwerts inwertgesetzter Natur auf. Ein weiterer Aspekt betrifft die normative Dimension von Naturbewertung: implizit werden dabei natürliche Produkte und Ökosystemdienstleistungen als primär wünschenswert erachtet. Dabei wird übersehen, dass nicht alles was aus der Natur kommt positive Effekte hat, wenn man z.B. an natürliche Krankheitserreger denkt.

Die Verhandlung von gesellschaftlichen Werten der Natur ist ein weitreichendes Unterfangen. Ein Foresight-Prozess, der besonders ethische, rechtliche und soziale Fragestellungen in den Vordergrund rückt und die Grundlage für einen breiteren gesellschaftlichen Diskurs zu langfristigen Entwicklungsmöglichkeiten und erwünschten Zukunftsvisionen für naturbasierte Industrielösungen schafft, wäre deshalb empfehlenswert. 


\section{Zitierte Literatur}

Duque, A. d. B., Antonielli, L., Sessitsch, A., Samad, A. und Compant, S., 2019, Mycolicibacterium strains interact positively with Serendipita (Piriformospora) indica for crop enhancement and biocontrol of pathogens, "Microbe-assisted crop production - opportunities, challenges and needs" (miCROPe 2019) Vienna, Austria.

Ganglberger, E., Sturm, T., Zahradnik, G. und Scherngell, T., 2017, Quantitative Indikatoren für die Biobasierte Industrie in Österreich, im Auftrag von: bmvit Bundesministerium für Verkehr, I. u. T., Wien.

Ojuederie, O. B. und Babalola, O. O., 2017, Microbial and Plant-Assisted Bioremediation of Heavy Metal Polluted Environments: A Review, International journal of environmental research and public health 14(12), 1504; auch veröffentlicht in: Int J Environ Res Public Health.

Schröter, M., van der Zanden, E. H., van Oudenhoven, A. P. E., Remme, R. P., Serna-Chavez, H. M., de Groot, R. S. und Opdam, P., 2014, Ecosystem Services as a Contested Concept: a Synthesis of Critique and Counter-Arguments, Conservation Letters 7(6), 514-523.

TEEB, 2010, The Economics of Ecosystems and Biodiversity: Mainstreaming the Economics of Nature: A synthesis of the approach, conclusions and recommendations of TEEB. 


\section{Illegaler Handel mit E-Schrott}

Mit zunehmender Digitalisierung des Alltags und der kürzer werdenden Lebensdauer (Stichwort: geplante Obsoleszenz) elektronischer Geräte, nimmt auch die Menge an elektronischem Abfall zu. Der Großteil des entstehenden Abfalls wird nicht fachgerecht am Entstehungsort entsorgt und in weiterer Folge recycelt, sondern in Länder exportiert, die geringere Arbeits- und Umweltschutzauflagen haben (Bakhiyi 2018). Das Basel Action Network (BAN) ${ }^{1}$ - eine NGO mit Sitz in Seattle, die sich der Problematik des elektronischen Abfalls verschrieben hat und eng mit der UN zusammenarbeitet - hat mittels versteckter GPS-Tracker aufgedeckt, dass der Abfall unter anderem nach Taiwan, China, Pakistan, Thailand, Kenia und weitere Schwellen- und Entwicklungsländer exportiert wird. ${ }^{2}$ Trotz der internationalen Konvention zur Regelung des Exports von gefährlichen Abfällen zwischen entwickelten Ländern und Schwellen- bzw. Entwicklungsländern (Basel-Konvention) und der europäischen Umsetzung dieser Konvention in der Waste-Shipment-Regulation (WSR), werden Schätzungen zufolge jährlich zwei Millionen Tonnen elektronischen Abfalls illegal aus Europa exportiert (Zoetman 2006). Der illegale Export von elektronischem Abfall ist für internationales organisiertes Verbrechen zu einem lukrativen Geschäftsmodell geworden, denn der Abfall enthält viele wertvolle Ressourcen, wie Edelmetalle (Gold, Silber) oder seltene Erden (Neodym, Yttrium oder Europium). Der weltweit größte Elektronikschrottplatz der Welt befindet sich in Guiyu (China). Aus einem Dorf, dessen Wirtschaft vormals auf dem Anbau von Reis basierte, ist mittlerweile die "Hauptstadt" des E-Schrotts geworden. Der Reisanbau ist aufgrund der hohen Verschmutzung nicht mehr möglich, denn um die Metalle zu extrahieren, die in den Elektrogeräten enthalten sind, werden diese verbrannt.

Das Thema E-Schrott ist für Österreich aufgrund mehrerer Aspekte als relevant zu betrachten. Einerseits kursiert gegenwärtig das Beispiel des ausgebrannten Tesla, dessen Entsorgung sich langwierig und mühsam gestaltete. ${ }^{3}$ Bei der Entsorgung von E-Autos Hersteller stärker in die Pflicht zu nehmen bzw. geeignete und u.U. gewinnbringende Entsorgungsprozesse (Akku-Pfand) einzurichten wäre langfristig wünschenswert. Andererseits schädigt die illegale Sammlung und anschließende Ausfuhr von Elektroaltgeräten die österreichische Volkswirtschaft, indem es zur Erlösverringerung für die Kommunen durch Verlust von Sekundärrohstoffen (Rohstoffe, die durch Recycling aus entsorgtem Material gewonnen werden) kommt. Obwohl Österreich die europäische Quotenvorgabe erfüllt, muss der Anteil an gesammelten Elektroaltgeräten ab 2019 wesentlich erhöht werden. Auch das Recycling von Elektroabfall, also das Rückgewinnen von wertvollen Ressourcen, hat das Potential, die nationale Wirtschaft zu fördern. Aufgrund bestehender Kompetenzen im Bereich

\footnotetext{
1 ban.org.

2 pbs.org/newshour/science/america-e-waste-gps-tracker-tells-all-earthfix.

3 diepresse.com/5725891/ausgebrannter-tesla-stellt-entsorgungswirtschaft-vorratsel.
} 
des Abfallmanagements und Recyclings scheinen hierfür die entsprechenden Voraussetzungen vorhanden zu sein. Dadurch würde auch der internationale und organisierte illegale Handel mit Elektroabfällen erschwert werden.

\section{Zitierte Quellen}

Bakhiyi, B., Gravel, S., Ceballos, D., Flynn, M. A. und Zayed, J., 2018, Has the question of e-waste opened a Pandora's box? An overview of unpredictable issues and challenges, Environment International 110, 173-192 sciencedirect.com/science/article/pii/S0160412017314708.

European Police Office 2015, Exploring Tomorrow's Organised Crime. europol.europa.eu/sites/default/files/documents/Europol_OrgCrimeRep ort_web-final.pdf.

Zoetman B. C. J. 2006, Global Waste Electrical and Electronic equipment (WEEE) streams estimates. Tias Business School and Telos, Brabant Centre for Sustainable development, Tilburg University. 


\section{Urban Mining 4.0}

\section{Zusammenfassung}

Bis zum Jahr 2050 wird sich die Zahl der Menschen, die weltweit in Städten wohnen, verdoppeln. Mit der steigenden Bevölkerung ist ein steigender Rohstoffverbrauch bei sinkender Verfügbarkeit von Ressourcen verbunden, doch die Städte sind damit zugleich Lagerstätten für eben diese Rohstoffe. ${ }^{1}$ Häuser und Infrastrukturen enthalten Baumaterialien und Metalle, die wiederverwertet werden können. Computer, Handys und Batterien gehören zu den Geräten, die Industriemetalle benötigen, die als Seltene Erden bezeichnet werden. Die Ursprungsquellen der Industriemetalle sind noch dazu in wenigen Ländern konzentriert, insbesondere in China, was eine politische und wirtschaftliche Abhängigkeit mit entsprechenden Risiken für die Wirtschaft bedeutet. Urban Mining gilt als Ansatz, die Lagerstätten in den Städten nutzbar zu machen (Binnemans et al. 2013). Wenn bisheriges Urban Mining systematisch mit Ansätzen der Kreislaufwirtschaft und der Internet- und Sensor-Vernetzung von Industrie 4.0 verbunden wird, kann daraus ein Urban Mining 4.0 entstehen.

\section{Überblick zum Thema}

Seit Jahrzehnten werden Bodenschätze in die Industriestaaten transferiert und zu Infrastrukturen, Häusern, Maschinen und elektronischen Geräten verbaut. Seit Rohstoffe in ihrer ursprünglichen Form weltweit knapper und teurer werden, wird die Stadt als neue Rohstoffquelle entdeckt. Das Konzept des Urban Mining bezieht sich darauf, Städte als Äquivalent zu einem Bergwerk zu begreifen. Rohstoffe, unter anderem wertvolle und seltene Stoffe müssten nicht mehr aus Boden und Gestein gewonnen werden, sondern aus alten Maschinen, aus ausgedienten Gebäuden, aus Mülldeponien und elektronischen Altgeräten. Diese Rohstoffe, die durch Aufarbeitung (Recycling) aus entsorgtem Material gewonnen werden, werden als Sekundärrohstoffe bezeichnet. Die verarbeiteten Metalle und insbesondere die Seltenen Erden aus den verarbeiteten Produkten wieder zu extrahieren, ist eine umfassende sozio-technische Herausforderung der nächsten Jahrzehnte.

In den Städten sind sowohl in intakten Gebäuden und der verbauten Infrastruktur als auch in den anfallenden Mengen an Bauschutt eine Vielzahl von Rohstoffen enthalten, die wiederverwertet werden könnten: Sowohl mineralische Materialien wie Beton, Ziegel und Keramik als auch Basismetalle wie Stahl, Aluminium und Kupfer. Dazu kommen Kunststoffe, Gips und Holz. Während bestehende Gebäude nicht von vornherein auf

umfassende sozio-technische Herausforderung der nächsten Jahrzehnte

\footnotetext{
1 Für einige Materialien wird davon ausgegangen, dass diese „anthropogenen Lager" bereits in der Größenordnung der Reserven (also der unter derzeitigen Bedingungen abbaubare Rohstoffvorkommen) in natürlichen Lagerstätten vorliegen. Vgl. die Studie aus dem Jahr 2019: nachhaltigwirtschaften.at/resources/sdz_pdf/berichte/schriftenreihe_2019-14_urban-mining.pdf.
} 
Urban Mining als interdisziplinärer Ansatz

vier Säulen des Urban Mining die Wiederverwertung ausgerichtet sind, werden heute bereits Gebäudekonzepte aus trennbaren Ressourcen entwickelt, die die vollständig wiederverwendbar oder kompostierbar sind. ${ }^{2}$

Elektroschrott (siehe Thema „Illegaler Handel mit E-Schrott“) fällt in steigendem Ausmaß an und droht zu einem globalen Problem von unüberschaubarem Ausmaß zu werden. Urban Mining wird zunehmend als effektive und effiziente Form gesehen, Edelmetalle aus Elektroschrott zu extrahieren. Während bei Autos das Recycling stark realisiert wird, ist dies bei Mobiltelefonen noch nicht der Fall, obwohl in Mobiltelefonen neben wenig bekannten seltenen Erden auch Kupfer und Kobalt, Silber, Gold und Palladium enthalten sind. In China wird Kupfer und Gold aus Elektroschrott bereits zu Kosten extrahiert, die mit denen des Abbaus von Erzen vergleichbar sind. Dieser Trend verweist auf ein hohes Potenzial der Verwertung von Elektroschrott, aber auch auf die Wirksamkeit von Urban Mining hin zu einer Transformation, in der die Kreislaufwirtschaft lineare Wirtschaftswege zurückdrängen könnte (Zeng et al. 2018).

Urban Mining verbindet als interdisziplinärer Ansatz Städteplanung, Produktentwicklung, Recycling und Forschung. Wenn wertvolle Rohstoffe nicht mehr aus dem Boden, sondern aus abrissreifen Gebäuden, aus Mülldeponien und aus elektronischen Geräten gewonnen werden, sind Konzepte gefragt, die ganze Städte als integrierte Recycling-Systeme adressieren können (Brunner 2011). In der Forschung gibt es Ansätze, die stetig wachsenden „städtischen Minen“ systematisch zu erschließen. Die Entwicklung neuer Recycling-Technologien ist dabei ebenso Gegenstand wie die aufwändige Erhebung urbaner Rohstofflager und ihrer Darstellung in Datenbanken. Es gibt bereits viele hochspezifische Ansätze in einzelnen Bereichen (Gebäude, Straßen, Haushaltsgeräte) und spezielle Verfahren für einzelne Stoffe. Sekundärrohstoffe werden umso konkurrenzfähiger, je avancierter die Methoden zu ihrer Gewinnung sind und je stärker das Design von Gütern, die Dokumentation der enthaltenen Rohstoffe, die Informationen über Lagerstätten und die Rückgewinnung aufeinander bezogen sind. Ob die Wiedergewinnung der Rohstoffe tatsächlich attraktiver wird, das rohstoffliche Recycling steigen wird und die Beseitigung und energetische Verwertung (Verbrennen) zurückgehen wird, ist eine Frage, die von den politischen Rahmenbedingungen abhängt.

Vielfach werden vier Säulen von Urban Mining ${ }^{3}$ identifiziert.

- Design ist die Säule, in der es darum geht, bereits im Entwurf und in der Realisierung von Konsum- und Industriegütern die Rückgewinnbarkeit der verwendeten Rohstoffe zu berücksichtigen. Dieser Bereich ist nationalstaatlich nur begrenzt gestaltbar, aber auf europäischer Ebene zum Teil zukunftsorientiert regulierbar.

\footnotetext{
2 nest-umar.net/. land-der-ideen.de/wettbewerbe/beyond-bauhaus/preistraeger/urban-mining-andrecycling-unit.

3 ooe-zukunftsakademie.at/UrbanMining_Standpunkt_Flyer.pdf.
} 
- Bei der Dokumentation der Rohstoffinformationen geht es darum, bei Gütern mit langer Lebensdauer Informationen bezüglich Art, Menge und Zusammensetzung über geeignete Systeme abrufbar zu machen; hier könnte von der Sensor-Vernetzung im Kontext von Industrie 4.0 sehr stark profitieren werden, z.B. in Zukunft über Smart-City-Konzepte. $^{4}$

- Im Hinblick auf die urbane Prospektion geht es wie im klassischen Bergbau darum, urbane Lagerstätten vor einer Erschließung zu lokalisieren und zu erkunden. Auch das ist mittels sensorischer Vernetzung und Online-Verfügbarkeit in weit höherem und günstigerem Maße möglich als in der Vergangenheit.

- Der Bereich der Technologien für Trennung und Rückgewinnung zielt darauf $a b$, wirtschaftliche Rückgewinnung und sekundäre Verarbeitung möglich zu machen. Es geht darum die physikalischen und chemischen Prozesse weiter zu entwickeln, die mit wenig stofflichem und energetischem Aufwand die Gewinnung der einzelnen Stoffe ermöglichen. In diesem Bereich wird in Zukunft ein hohes Maß an Automatisierung greifen können.

Die Säulen des Urban Mining verweisen darauf, dass die wichtigen Bereiche noch vielfach nebeneinanderstehen, aber über Digitalisierung und Industrie 4.0 eng verbunden und konzeptionell viel stärker miteinander verbunden werden können.

\section{Relevanz des Themas für das Parlament und für Österreich}

In Österreich ist Urban Mining konzeptionell stark verankert und ein deutlich prominenteres Thema als in anderen Ländern. Forschungsprojekte ${ }^{5}$, ein Kompetenzzentrum ${ }^{6}$, ein Blog$^{7}$ mit Reichweite bei den Stakeholdern, starke Awareness und hohes Interesse bilden eine gute Ausgangslage. Das Thema ist bisher ein Spezialthema dessen breite gesellschaftliche Dimension nicht im Vordergrund steht.

Für eine umfassende Wirksamkeit eines „städtischen Bergbaus“ ist jedoch eine mehrfache Einbindung des Urban Mining notwendig: Zum einen in die Kreislaufwirtschaft, zum zweiten die Verbindung mit Industrie 4.0 durch eine umfassende Digitalisierung sowie den Einsatz von Sensorik und zum dritten durch ineinandergreifende soziale, organisatorische Adaption in Innovationsprozessen selbst.

\footnotetext{
4 Vgl. wien.orf.at/news/stories/2794516.

5 Siehe: wien.orf.at/news/stories/2794516/.

6 science.apa.at/dossier/Projekt_ReWaste_4_0_erforscht_Abfallbehandlung_der

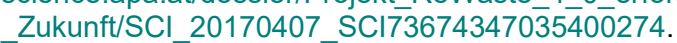

7 urbanmining.at/about.
} 


\section{Vorschlag weiteres Vorgehen}

Eine mögliche Langstudie könnte einen breiten systematischen Überblick zu den aktuellen Initiativen, möglichen technologischen Entwicklungen und ihrer systematischen Integration und Kopplung untersuchen. Der internationale und vor allem der europäische Stand der Forschung zu den Perspektiven von Urban Mining im Zeitalter von Digitalisierung, Sensorik und Online-Vernetzung könnte die Grundlage bieten, um die bestehenden Ansätze in Österreich zu unterstützen, die verschiedenen Dimensionen und Bereiche (u.a. Abfallwirtschaft, Industrie, Städte, Forschung) und relevante Stakeholder-Gruppen (auch neue) an der Entwicklung von Zukunftsoptionen für Urban Mining 4.0 zu beteiligen.

\section{Zentrale weiterführende Quellen}

Binnemans, K., Jones, P. T., Blanpain, B., Van Gerven, T., Yang, Y. X., Walton, A. und Buchert, M., 2013, Recycling of rare earths: a critical review, Journal of Cleaner Production 51, 1-22 < <Go to ISI>://WOS:000320419500001 >; auch veröffentlicht in: J. Clean Prod. Brunner, P. H., 2011, Urban Mining A Contribution to Reindustrializing the City, Journal of Industrial Ecology 15(3), 339-341 < <Go to ISI>://WOS:000291222200002 >; auch veröffentlicht in: J. Ind. Ecol.

Zeng, X., Mathews, J. A. und Li, J., 2018, Urban Mining of E-Waste is Becoming More Cost-Effective Than Virgin Mining, Environmental Science \& Technology 52(8), 4835-4841 < doi.org/10.1021/acs.est.7b04909 >. 


\section{Lieferung auf der letzten Meile unter Nachhaltigkeitsgesichtspunkten}

Unter der "Letzten Meile“ versteht man im Personenverkehr oder in der Warenlogistik den letzten Abschnitt einer Wegstrecke zur geplanten Destination. Gerade in den letzten Jahren hat vor allem der Buisiness to Customer (B2C) Warenversand stark zugenommen, was letztlich auf das hohe Wachstum im Bereich des Distanzhandels zurückzuführen ist. Einer Studie des österreichischen Handelsverbands zufolge, gaben die ÖsterreicherInnen im Jahr 2017 7,6 Mrd. € über den Distanzhandel aus (KMU Forschung Austria 2017). Viele dieser erworbenen Waren müssen versendet werden, was schließlich auch zur Problematik der Letzten Meile führt: Die Paketzustellung erfolgt oft zu Zeiten in denen niemand anwesend ist, um die Sendung zu empfangen. Diese erfolglosen Zustellungen werden wieder in das Paketdepot zurückgebracht bis sie neuerdings zugestellt werden können. Das hat nicht nur ökologische (erhöhtes Verkehrsaufkommen), sondern auch ökonomische (Staus durch Lieferwagen) und soziale Folgen (geringere Lebensqualität aufgrund erhöhtem Verkehrsaufkommen) (Allen et al. 2000). Verknüpft mit dem Trend, dass immer mehr Menschen in Städten leben, führt das auch dazu, dass das Stadtbild zusehends von Zustelldienstfahrzeugen geprägt ist. Innovative Konzepte, um damit umzugehen, sind zum Beispiel, dass der Kunde oder die Kundin in so genannten Paketabholstationen das Paket abholen kommt, oder innovative Zustellsysteme, wie zum Beispiel Lastenfahrräder $^{1}$, V-Feather-Fahrzeuge oder Drohnen (siehe dazu Thema "Lieferdrohnen“). Bei V-Feather-Fahrzeugen handelt es sich um Elektroautos, die modular aufgebaut sind. Die Fahrerkabine kann sich an verschiedene Frachtmodule andocken (Kühlwagen, Gefahrengut, Paketwagen etc.) (Slabinac 2015). Abgesehen von der Problematik der Letzten Meile, sind auch innovative Lösungen für Verpackungssysteme notwendig, denn bis jetzt sind die Versandverpackungen so gestaltet, dass man sie im Restmüll deponiert anstatt wieder zu verwenden. Es gibt bereits innovative Ansätze dazu, wie zum Beispiel die Nutzung von biologisch abbaubaren Verpackungsmaterialien (siehe dazu Thema „Biobasierte Zukunftsmaterialien"), jedoch hat sich das noch nicht durchgesetzt. Da Lieferdienste in Zukunft weiter zunehmen werden, besteht aus Sicht von F\&E und Politik Gestaltungsspielraum, um innovative Lösungsansätze für diese Herausforderungen zu entwickeln.

\footnotetext{
1 In Wien wird ab November 2019 im Rahmen des Forschungsprojekts RemiHub getestet, wie Öffi-Flächen, in diesem Fall eine Busgarage, künftig als urbane Logistik-Hubs für die städtische Paketzustellung per Lastenfahrrad genutzt werden könnten. Siehe futurezone.at/science/wie-oeffi-garagen-das-groessteproblem-der-paketzustellung-loesen-koennen/400577417.
} 


\section{Zitierte Quellen}

KMU Forschung Austria, 2017, E-Commerce-Studie Österreich 2017 Konsumentenverhalten im Distanzhandel.

Allen, J., Anderson, S., Browne, M. \& Jones, P. 2000, A framework for considering policies to encourage sustainable urban freight traffic and goods/service flows - Report 2: Current goods and service operations in urban areas. London: Transport Study Group, Universität Westminster.

Slabinac, M, 2015, Innovative Solutions for a "Last-Mile“ Delivery A European Experience, 15th international scientific conference Business Logistics in Modern Management, 15. Okt. 2015, Osijek Kroatien. 


\section{Zwischenspeicher der Zukunft für elektrische Energie}

Die Dekarbonisierung des Energiesystems und die dazu notwendige Energiewende bedürfen großer systemischer Anpassungen in der Energiebereitstellung. Regenerative Stromerzeugung wie Windkraft oder Photovoltaik ist abhängig von der Jahres- bzw. Tageszeit (Cebulla et al. 2017). Erschwerend kommt die Tatsache hinzu, dass die Nutzungsprofile - also der Bedarf an Energie im täglichen bzw. saisonalen Verlauf - nicht immer den Produktionsprofilen entsprechen. Auch die oft vorherrschende räumliche Distanz zwischen Erzeugung und Verbrauch der elektrischen Energie, ist eine infrastrukturelle Herausforderung für die Dekarbonisierung. Eine mögliche Lösung für diese Herausforderung, könnten Speichertechnologien sein, die Energieüberschüsse zwischenspeichern wenn sie entstehen. Diese Energie-Zwischenspeicher ${ }^{1}$ wären somit eine Senke für temporäre Energie-Überschüsse und sie würden damit einen wesentlichen Beitrag zur Flexibilisierung des Netzes liefern (EASAC 2017). In Österreich übernehmen zurzeit Pumpspeicherkraftwerke diese wichtige Speicherfunktion, jedoch ist die Situation der Speicherkraftwerke in Österreich aufgrund der vorherrschenden Rahmenbedingungen (niedrige Strompreise, geringe untertägige Preisunterschiede) als prekär zu bezeichnen ${ }^{2}$. In Zukunft könnte innen jedoch eine größere Rolle im Hinblick auf den Ausgleich bei Stromerzeugungsüberschüssen durch erneuerbare Energien zukommen. Weitere alternative und vielversprechende Methoden zur Speicherung von elektrischer Energie sind elektrochemische Speichersysteme (Zhang et al. 2018). Hierbei gibt es unterschiedliche technische Möglichkeiten die elektrochemischen Speichersysteme umzusetzen z.B. Aluminium-lonen-Batterien, Vanadium-basierte Flow-Batterien, Natrium-Schwefel-Batterien oder Lithium-Ionen-Batterien. Innovative Speichertechnologien der Zukunft weisen ein hohes strategisches Potential auf, um neue nationale Märkte zu erschließen ${ }^{3}$. Daher wird international viel Grundlagenforschung in diesem Bereich betrieben (Zhang et al. 2018). Auch im Hinblick auf das Katastrophenmanagement kann die Technologie von elektrischen Zwischenspeichern einen wesentlichen Beitrag leisten, wie ein Beispiel aus Puerto Rico zeigt ${ }^{4}$ : TESLA hat ihre Speichersysteme (Lithium-Ionen-Batterien) kurz nach dem Hurrikan Maria, der dazu führte, dass $97 \%$ von Puerto Rico ohne Stromversorgung waren, zur Verfügung gestellt. Somit konnte zumindest die kritischen Infrastrukturen aufrechterhalten werden.

1 Genaugenommen kann elektrischer Strom nicht gespeichert werden, sondern nur in eine andere Energieform umgewandelt und bei Bedarf rückverstromt werden.

2 tugraz.at/fileadmin/user_upload/Events/Eninnov2012/files/pr/PR_Kloess.pdf.

3 isi.fraunhofer.de/content/dam/isi/dokumente/cct/2016/EnergiespeicherMonitoring-2016.pdf.

4 futurism.com/tesla-is-shipping-hundreds-of-powerwall-batteries-to-puerto-rico/. 
In Österreich sind Energiespeicher seit einigen Jahren sowohl in F\&E als auch zuletzt in der Politik zu einem wichtigen Thema geworden. Der Klima- und Energiefonds (KLIEN) hat mit der „Speicherinitiative“5 ab Herbst 2015 eine Aufbereitung des Themas mit umfassender StakeholderBeteiligung geleistet. Diese Ergebnisse sind mittlerweile in zahlreiche technische F\&E-Projekte, gefördert durch den KLIEN, gemündet. 2018 wurde für das BMVIT die Technologie-Roadmap „Energiespeichersysteme in und aus Österreich" erarbeitet (Friedl et al. 2018). Das Parlament hat in der Folge die Arge ITA-AIT Parlament mit einer Überblicksstudie zu diesem Thema aus Perspektive von Foresight und Technikfolgenabschätzung beauftragt, deren Ergebnisse im Sommer 2019 vorliegen werden. ${ }^{6}$

\section{Zitierte Quellen}

Cebulla, F., Naegler, T. und Pohl, M., 2017, Electrical energy storage in highly renewable European energy systems: Capacity requirements, spatial distribution, and storage dispatch, Journal of Energy Storage 14, 211223, sciencedirect.com/science/article/pii/S2352152X17302815.

EASAC 2017, Valuing dedicated storage in electricity grids, EASAC policy report 33, easac.eu/fileadmin/PDF_s/reports_statements/Electricity_Storage/EAS AC_Electricity_Web_low_res_30_June.pdf.

Friedl W., Wilk V., Popp H., Kubeczko K., 2018, Technologie-Roadmap „Energiespeichersysteme in und aus Österreich“, Klima- und Energiefonds (Herausgeber), Wien.

Zhang, C., Wei, Y.-L., Cao, P.-F. und Lin, M.-C., 2018, Energy storage system: Current studies on batteries and power condition system, Renewable and Sustainable Energy Reviews 82, 3091-3106, sciencedirect.com/science/article/pii/S1364032117314077.

5 Website: speicherinitiative.at; Abschlussbericht der Startphase (Juni 2016): speicherinitiative.at/assets/Uploads/Abschlussbericht-

Startphase/Speicherinitiative-Abschlussbericht-Startphase-lowres.pdf.

6 oeaw.ac.at/ita/projekte/wie-wir-in-zukunft-elektrische-energiespeichern/ueberblick/. 


\section{Grüne Wasserstoffproduktion 2.0}

Die nächste Generation der Hochtemperatur-Elektrolyse zur Erzeugung von Wasserstoff verspricht gesteigerte Effizienz und Nachhaltigkeit. Dabei wird Wasserstoff mittels Hochtemperatur-Elektrolyse bei $800^{\circ} \mathrm{C}$ gewonnen (Power-to-Gas-Technologie) und in Festoxidzellen (rSOEC - reversible Solid Oxide Electrolysis Cell) gespeichert. Die rSOEC-Technologie ermöglicht eine zweifache Art der Nutzung einer Wasserstoffproduktionsanlage: einerseits kann Wasserstoff produziert und gespeichert werden, andererseits kann die Anlage innerhalb kurzer Zeit von Wasserstoff auf Strom- und Wärmeproduktion umgestellt werden. Zu den Vorteilen des neuen Verfahrens zählen dabei der höhere Wirkungsgrad von Hochtemperatur-Brennstoffzellen bei der Wasserstofferzeugung ( $>50$ Prozent), die mögliche Verwendung von unedleren und kostengünstigeren Materialien als für Niedrigtemperatur-Brennstoffzellen, und die Rolle der Speichertechnologie zum Ausgleich von Angebots-/Nachfrageschwankungen, v.a. im Hinblick auf punktuelle Überschüsse aus erneuerbarer Energieproduktion. Mithilfe der rSOEC-Technologie kann in weiterer Folge ein Synthesegas erzeugt werden, dessen chemische Eigenschaften sehr gezielt einstellbar sind. Diese maßgeschneiderten Kraftstoffe verbrennen in Motoren und Turbinen quasi schadstoff- und rückstandsfrei. Derzeit befindet sich die Technologie in der Testphase. Das deutsche Unternehmen Sunfire hat 2014 eine Pilotanlage in Betrieb genommen ${ }^{1}$ und das Forschungszentrum Jülich beforscht den Lebenszyklus der neuen HochtemperaturBrennstoffzellen (Frey et al. 2018). Das zukünftige Potenzial der Technologie steht eng in Zusammenhang mit gegenwärtigen Veränderungsprozessen in Energiesystemen.

\section{Zitierte Literatur}

Frey, C. E., Fang, Q., Sebold, D., Blum, L. und Menzler, N. H., 2018, A Detailed Post Mortem Analysis of Solid Oxide Electrolyzer Cells after Long-Term Stack Operation, Journal of The Electrochemical Society 165(5), F357-F364.

1 sunfire.de/de/unternehmen/news/detail/naechste-generation-derhochtemperatur-elektrolyse-gestartet. 


\section{Plus Energie Quartiere: Zukunft der lokalen Energieversorgung?}

Neue Energielösungen für Städte und Stadtviertel sind dringend nötig. In Europa und Österreich werden daher sogenannte Plus Energie Quartiere als zukunftsfähige Ansätze zur Umsetzung der klima- und energiepolitischen Ziele betrachtet. Dementsprechend wird im Umsetzungsplan der österreichischen Energieforschungsinitiative in der Klima- und Energiestrategie deren Entwicklung als Mission festgelegt und soll bis 2025 in drei Modellstädten realisiert und getestet werden ${ }^{1}$. Schon jetzt gibt es Forschungsförderungsprogramme in Österreich ${ }^{2}$ und in Europa ${ }^{3}$, um Planung, Aufbau und Betrieb von Plus Energie Quartieren (Positive Energy Districts) voranzutreiben. Ein Plus Energie Quartier ist ein Gebiet mit einer jährlichen lokalen Überschussproduktion erneuerbarer Energien, einem jährlichen Netto-Nullenergieimport und Netto-Null- $\mathrm{CO}_{2}$-Emissionen. Gleichzeitig sollen die so aufgewerteten Stadtviertel lebenswert, leistbar und inkludierend sein. Ausreichende Erzeugung erneuerbarer Energie, Steigerung der Energieeffizienz und die bestmögliche Flexibilität in der Energienutzung sind die drei Säulen der Energiestrategie. Durch entsprechende Optimierung sollen lokal sogar Energieüberschüsse in ein städtisches bzw. regionales Energiesystem integriert werden können und Energieimporte zur Wahrung der Versorgungssicherheit minimiert werden. Ein wirtschafts- und innovationspolitisches Ziel ist es, durch Replizieren derartiger urbaner Gebiete Österreich bzw. Europa zum globalen Vorbild für die Dekarbonisierung zu machen und vom damit verbundenen Know-how langfristig auch ökonomisch zu profitieren. Für die Entstehung von Plus Energie Quartieren braucht es eine enge Kooperation zwischen Politik (insbesondere Stadt-, Energie- und Klima-, Sektor- sowie Forschungs- und Innovationspolitik), städtischen Verwaltungen, Infrastrukturbetreibern, Industrie, Forschungsorganisationen und zivilgesellschaftlichen Akteure. Gemeinsame Visionen einer nachhaltigen Urbanisierung, die von den oben genannten Akteursgruppen und Stakeholdern geteilt werden, sollen dabei die Basis einer orchestrierten Anstrengung zur Realisierung von Plus Energie Quartieren bilden. Die Umsetzung erfordert die Interaktion und Kollaboration aller Akteure, einschließlich der Nutzerlnnen, sowie das Zusammenspiel zwischen den technologischen Komponenten der Gebäudeinfrastrukturen und des regionalen Energie- sowie Mobilitätssystems, gestützt durch intelligente Informations- und Kommunikationslösungen. Die erforderlichen komplexen Innovations- und Planungsprozesse können in Living Labs und Reallaboren stattfinden.

\footnotetext{
1 BMVIT 2019: Umsetzungsplan zur Energieforschungsinitiative in der Klima- und Energiestrategie, BMVIT in Kooperation mit WKO und Klima- und Energiefonds. ikes-umsetzungsplan.at.

2 ffg.at/stadt-der-zukunft-programmlinie.

3 jpi-urbaneurope.eu/ped/.
} 
Durch die Wahl von Technologien und Geschäftsmodellen, die Klärung von Eigentumsverhältnissen sowie durch institutionelle Rahmenbedingungen, die die Handlungsspielräume der beteiligten Akteure festlegen, können fruchtbare Pfadabhängigkeiten geschaffen und Risiken determiniert werden. Regulatorische Experimentierräume (siehe Regulatorische Experimentierräume) und die Nutzung neuer Finanzierungsmodelle könnten Österreich eine führende Position in diesem Bereich verschaffen und dadurch auch entscheidende Wettbewerbsvorteile bewirken. Allerdings herrschen unausgewogene Machtverhältnisse zwischen den verschiedenen Stakeholdern vor, die durch ein Übergewicht von Akteuren aus Industrie, Verwaltung und Politik gekennzeichnet sind. Die Stärkung zivilgesellschaftlicher Akteure in ihrer umfassenden Rolle als Bürgerlnnen und die aktive Einbindung von Menschen in ihren unterschiedlichen Rollen als BewohnerInnen, MieterInnen, EigentümerInnen, NutzerInnen oder ProduzentInnen von Energie (z.B. durch lokale Energiegemeinschaften) in die entsprechenden Governance-Prozesse wäre empfehlenswert und könnte langfristig zur Erhöhung der gesellschaftlichen Akzeptanz von Plus Energie Quartieren beitragen und Umsetzungsrisiken minimieren. 


\section{Straße in die Zukunft: Smarte Infrastruktur?}

Die Straße der Zukunft ist gepflastert mit speziellen Solarzellen, die integriert in die Straßenoberfläche Strom erzeugen. Um Straßenneubau zu vermeiden, können die Solarzellen kostengünstig einfach aufgeklebt werden und sind robust genug, auch schweren LKWs standzuhalten. Mithilfe von selbstreinigenden und selbstheilenden Supermaterialien können Beeinträchtigungen der Solaroberfläche durch Schmutz oder mechanische Einwirkung vermieden werden (siehe dazu auch Intelligente Oberflächen oder Bioinspirierte Materialien). Der erzeugte Strom wird zukünftig unmittelbar über kabellose Ladeplatten, die an der Straßenoberfläche angebracht werden können, in die Elektroautos übertragen. Die dazu notwendige Technologie liefert das österreichische Start-up Easelink mit dem massentauglichen Matrix-Charging-System, das gegenwärtig weiterentwickelt, getestet und für den Einsatz in Städten vorbereitet wird. ${ }^{1}$ Diese Vision der Solarstraße wird bereits in mehreren Ländern erprobt: in den Niederlanden gibt es seit 2014 einen Solarradweg2; in Frankreich wurde 2016 eine $1 \mathrm{~km}$-lange Solarstraße getestet und in China wurde 2017 die größte Solarstraße der Welt $(1,8 \mathrm{~km})^{3}$ eröffnet. Während Solarradwege erste Erfolge in Hinblick auf die erzeugte Energie verbuchen (erwarteter Jahresertrag 50 bis $70 \mathrm{kWh}$ pro $\mathrm{m}^{2}$, tatsächlicher Jahresertrag $73 \mathrm{kWh}$ pro $\mathrm{m}^{2}$ ), erweisen sich Solarstraßen in mehrfacher Hinsicht als Flop. Sie erzeugen weniger Energie als erwartet (was vor allem der horizontalen Lage der Solarmodule und der Abschattung durch Fahrzeuge zuzuschreiben ist), was wiederrum negativ auf die Preis-/Leistungsrelation wirkt. Darüber hinaus sind Solarstraßen schadensanfälliger und lärmintensiv; je nach Oberflächenbeschaffenheit der neuartigen Straßenbeläge können auch Sicherheitsprobleme bspw. in Hinblick auf Rutschfestigkeit auftreten. ${ }^{4}$ Eine weitere Herausforderung im Zusammenhang mit smarter, zukünftiger Mobilitätsinfrastruktur tritt derzeit in Österreich besonders deutlich zutage: die Entsorgung von Elektroautos ist problematisch. Der Fall eines ausgebrannten Tesla ${ }^{5}$, der wochenlang nicht entsorgt werden konnte, da niemand die Verantwortung für die Entsorgung der großen LithiumIonen-Akkus die als Sondermüll gelten, übernehmen wollte, ist ein eindrückliches Beispiel. Das bei der großräumigen Einführung von neuer, smarter Infrastruktur ein Prozess zur Antizipation zukünftiger Herausforderungen sinnvoll ist, nicht zuletzt um Zuständigkeiten und Verantwortlichkeiten vorab auszuloten, liegt auf der Hand.

1 derstandard.at/story/2000110982379/wie-man-elektroautos-in-zukunft-ladenkoennte.

2 spiegel.de/reise/aktuell/solarradweg-in-den-niederlanden-solarzellen-unter-denreifen-a-998080.html.

3 geo.de/natur/oekologie/20108-rtkl-energie-china-entsteht-die-groesstesolarstrasse-der-welt-was-kann-sie.

4 theconversation.com/solar-freakin-roadways-why-the-future-of-this-technologymay-not-be-so-bright-51304.

5 derstandard.at/story/2000111333258/posse-um-entsorgung-von-unfall-tesla-intirol-nach-sechs. 


\section{Offene Mobilitätsplattformen zur Unterstützung der Verkehrswende?}

Eine Technologie für den Bereich Mobilität und Verkehr, die schon seit einigen Jahren Aufmerksamkeit erregt, sind echtzeitbasierte Verkehrs- und Reiseinformationssysteme. Eine Vielzahl an Mobilitätsplattformen bündeln inzwischen diese Dienste. Verschiedene Akteure stellen dafür Daten zur Verfügung; auf Grundlage dieser Daten werden mit Hilfe intelligenter Algorithmen individuelle Reiserouten mit verschiedenen Verkehrsmitteln berechnet. Die Routenvorschläge können miteinander verglichen und die jeweils günstigste Option gewählt werden. Doch zumeist sind die Alternativen und Optionen begrenzt, da sich mittlerweile Pfadabhängigkeiten ergeben haben, je nachdem in welchem Kontext und von welchen Akteuren die Plattformen entwickelt wurden.

Mobilitätsplattformen bieten einen Rahmen zur Koordination und Abstimmung von Mobilitätsdienstleistungen. Sie stehen theoretisch als Ideal für ein neues Mobilitätsverständnis: der sich fortbewegende Mensch und nicht mehr der Verkehrsträger steht im Zentrum. Die damit verbundene sozio-technischen Zukunftsvorstellung stellt ein Verkehrssystem in Aussicht, in dem mit derselben Verfügbarkeit (wie bisher mit dem eigenen Automobil) Mobilitätsdienstleistungen (Mobility as a Service, MaaS) zur Verfügung stehen. Umfassende, offene und interoperable Mobilitätsplattformen ermöglichen konzeptionell ein anderes Mobilitäts- und Verkehrsverhalten. In dichtbesiedelten Räumen, in denen sowohl ein öffentliches Verkehrsangebot, als auch verschiedene Sharing-Angebote zur Verfügung stehen, lässt sich sogar auf den Besitz des eigenen Automobils verzichten. Eine Verringerung des MIV wäre einer der wesentlichen Faktoren zur Reduzierung der negativen Umweltwirkungen des Verkehrs. Doch das Nebeneinander und die experimentelle Vielfalt von privaten, städtischen und infrastrukturgetriebenen Plattformen hat praktisch dazu geführt, dass das Ideal nachhaltiger Mobilität nicht erreicht werden kann, der Nutzen durch das Nebeneinander der Ansätze sinkt.

Das wirtschaftliche Potenzial von Mobilitätsplattformen ist für die Betreiber hoch. Wenn verschiedene Mobilitätsdienstleistungen auf einer Plattform integriert und attraktive multimodale Reisemöglichkeiten vermittelt werden, basiert das Geschäftsmodell darauf, dass Plattformbetreiber von Verkehrsunternehmen und anderen Anbietern, Gebühren für ihre Vermittlungsdienste erhalten. Gleichzeitig kann der Betreiber Daten erheben (v.a. Bewegungsdaten sind wirtschaftlich wertvoll).

Auch von kommunalen und regionalen Akteuren werden Mobilitätsplattformen angeboten. Diese Initiativen verfolgen oft verkehrs- und klimapolitische Ziele. Große Städte, wie z.B. Wien, haben inzwischen eigene Gesellschaften ausgegründet, die Mobilitätsplattformen vermarkten. Andere Städte haben dagegen eine Open-Data-Strategie gewählt. Lokale ITEntwickler werden dazu angeregt, kostengünstige Plattformlösungen um- 
zusetzen. Die Entwicklung von Mobilitätsplattformen wird aber auch über Innovationsfördermaßnahmen zur Digitalisierung des öffentlichen Verkehrs unterstützt.

Mobilitätsplattformen sind hinsichtlich ihrer gesellschaftlichen Konsequenzen ambivalent. Aus technisch-organisatorischer Perspektive verbessern sich die Möglichkeiten sich multimodal fortzubewegen. Der wirtschaftliche Erfolg von Mobilitätsplattformen hat dazu geführt, dass immer mehr internationale Akteure auf diesen Markt drängen. Dadurch verändert sich die Marktorganisation. Plattformen profitieren im Wesentlichen von der Vermittlung von Verkehrsdienstleistungen. Subunternehmen übernehmen die Fahrdienste. Eine Folge ist die Prekarisierung von Arbeitsverhältnissen. Die Zunahme verschiedener Mobilitätsdienstleistungsanbieter hat zu einem erweiterten Angebot geführt. Zugleich entstand ein schwer durchschaubares Nebeneinander von Anbietern.

Im Zuge der Digitalisierung haben sich die Angebote so vervielfacht, dass das System durch parallele Plattformen fragmentiert wird. Diesem Trend kann durch die Entwicklung eines Rahmens entgegengewirkt werden. Dabei könnte durch Einbindung der relevanten Akteure festgestellt werden, welchen Anforderungen und Prinzipien eine zukunftsorientiere, interoperable und übergreifende Plattform im Bereich der Mobilität (in Städten und städteübergreifend) entsprechen muss und welche staatlichen Rahmenbedingungen dafür die Voraussetzung sind. Das Nebeneinander und die Vielfalt der Plattformen bedeutet ein hohes Maß an Wissen und Erfahrungen der lokalen und regionalen Akteure, sowie der Infrastrukturbetreiber. Da inzwischen die Grenze und der abnehmende Nutzen der fragmentierten Plattformlandschaft deutlich wird, könnte hier eine Initiative auf Bundesebene auf der Basis der bisherigen Erfahrung, die Grundlagen für offene und interoperable Mobilitätsplattformen zur Unterstützung der Verkehrswende bilden. 


\section{Zukunft Lieferdrohnen?}

\section{Zusammenfassung}

Die von etlichen Firmen weltweit propagierte Vision eines drohnenbasierten Lieferverkehrs wäre nicht voraussetzungslos: Viele regulative und technische Hürden müssten noch genommen werden, um sie überhaupt Wirklichkeit werden zu lassen. Aufgrund der großen Eingriffstiefe dieser Technologieentwicklung - immerhin würde sich der uns umgebende Luftraum, der bislang nur von Vögeln und gelegentlichen Hubschraubern benutzt wird, gravierend ändern - stellen sich eine Reihe von typischen Fragen der Technikfolgenabschätzung (TA): Bestehen ethische oder Sicherheitsbedenken? Gibt es Umweltrisiken? Kann die Technologie für kriminelle oder terroristische Zwecke missbraucht werden? Besteht ein gesellschaftliches Konfliktpotenzial angesichts unterschiedlicher Interessen (z.B. Lärm, Privatsphäre, Luftraumverwendung)? Reicht die aktuelle Regulierung aus oder müssen neue Regeln geschaffen werden? Auch in Österreich gibt es bereits Experimente mit Lieferdrohnen. Die wissenschaftliche Basis für politische Entscheidungen fehlt jedoch noch weitgehend, ein öffentlicher Diskurs zur gesellschaftlichen Kosten-Nutzen-Abwägung ist noch nicht geführt.

\section{Überblick zum Thema}

War das Thema Drohnen vor Jahren noch vom Militär dominiert, ist es längst im zivilen Bereich und im Alltag vieler angekommen. Millionen Spielzeugdrohnen sind weltweit im Einsatz und wir haben uns an atemberaubende Filmaufnahmen aus bisher ungeahnten Perspektiven gewöhnt. Immer öfter begegnen uns auch Überwachungsdrohnen, viele haben schon einen Videoclip eines "Drohnen-Balletts" gesehen oder beobachtet, wie sich eine Touristin mit einem "fliegenden Selfie-Stick" filmt. In vielen Bereichen werden Pilotversuche durchgeführt, um den Nutzen von Drohnen zu testen, etwa in der Landwirtschaft, im humanitären und medizinischen Bereich, bei der Überprüfung von Anlagen, im Vermessungswesen, im Journalismus, im Tourismus und nicht zuletzt in der Forschung, um nur ein paar Beispiele zu nennen.

Schließlich arbeiten die großen Online-Händler, einige Postunternehmen und zahlreiche Startups weltweit darauf hin, Güter des täglichen Bedarfs durch die Lüfte zu liefern (siehe auch Thema „Letzte Meile"). Der Nutzen für die Kunden und Kundlnnen könnte darin bestehen, ihre bestellten Güter noch viel schneller und auch an Orte geliefert zu bekommen, die bislang nicht oder aus logistischen Gründen nur selten beliefert werden können. Für die Warenanbieter könnte dies neue bzw. geographisch ausgeweitete Märkte und potenziell einen Imagegewinn bedeuten. Für die Zustellfirmen würde sich die Struktur der Personal- und Wegekosten voraussichtlich verbessern. 
zwei mögliche Szenarien einer Zukunft mit Lieferdrohnen

Sicherheit und Umweltaspekte

ethische Fragen
Um allerdings diese Vision eines drohnenbasierten Lieferverkehrs zu realisieren, müsste noch viele regulative und technische Hürden genommen werden, und vermutlich werden manche Aspekte auch auf Widerstand in der Bevölkerung treffen. Damit Lieferungen „durch die Luft“ ökonomisch machbar sind, müssen die Drohnen autonom fliegen können, also ohne Piloten am Boden. Dafür müssen noch eine Reihe technischer Herausforderungen gemeistert werden. Diese reichen von Gewichts-, Reichweitenund Wetterproblemen bis zur Optimierung der Sensor- und Ausweichtechnologien. Prinzipiell dürfte davon ausgegangen werden können, dass es nur eine Frage der Zeit ist, bis diese Probleme gelöst sind, weil hier viele Unternehmen voranschreiten wollen. Darüber hinaus muss auch die nötige Infrastruktur am Boden bereitgestellt werden, etwa Landeplätze.

Die rechtlichen Voraussetzungen für autonom fliegende Drohnen sind in Europa erst in Ausarbeitung. In Österreich können daher Lieferdrohnen bislang nur mit Spezialgenehmigung der Luftfahrtbehörde getestet werden, eine Dauerbewilligung ist noch nicht möglich - womit ein guter Zeitpunkt ist, darüber nachzudenken, in welchem Umfang dies überhaupt möglich gemacht werden soll und kann.

Es können zumindest zwei Szenarien unterschieden werden, die mögliche Zukünfte mit drohnenbasierten Lieferungen beschreiben: (1) In einem Szenario würden Drohnen alle möglichen Güter des täglichen Bedarfs liefern. Dazu gibt es weltweit bereits vielfach Beispiele (Pizza in Neuseeland, Fast Food in Island, Kaffee in der Schweiz u.v.m.). Insbesondere die Firma Amazon hat bereits vor ein paar Jahren ihre Produktidee „Prime Air" lanciert, allerdings noch nicht verwirklicht. (2) In einem zweiten Szenario würden keine Massen- sondern Nischenmärkte bedient, etwa Speziallieferungen zu schwer zugänglichen Orten (Nebentäler, Almen, Inseln, im Katastrophenfall) oder bei besonderer Dringlichkeit, etwa im medizinischen Bereich (Blutproben, Medikamente, Organspenden) durchgeführt. Dieses Szenario wird in Afrika derzeit gerade vielerorts Wirklichkeit.

Eine erste Abschätzung bzw. vorläufige Themensammlung (ITA 2018) vor dem Hintergrund dieser beiden Szenarien kommt zum Schluss, dass noch eine Reihe von wichtigen Fragen gesellschaftlich und politisch zu klären sind, bevor Drohnen Teil der Logistikkette werden könnten. Zumindest folgende Aspekte sind relevant:

Abgesehen davon, dass abstürzende Drohnen Verletzungen verursachen können, könnten bestimmte Ladungen dabei auch Verseuchungen hervorrufen. Drohnenlieferungen können Wildtiere stören (Vorbeiflug, Lärm); abstürzende Pakete oder Drohnen können die Umwelt verschmutzen; eine Lebenszyklusanalyse und eine Untersuchung des Energieverbrauchs müssten durchgeführt werden.

Bekannt aus der Diskussion zu autonomen Kraftfahrzeugen stellen sich auch bei autonomen Drohnen ähnliche Fragen, da vorab programmierte Algorithmen im Fall von Unfallsituationen ethische Entscheidungen treffen müssen. Weiters stellt sich etwa die Frage, ob dieser Service für alle offen sein muss. 
Der Markt für Arbeitskräfte im Endkundenlieferverkehr, der im Zuge des Online-Shoppings massiv gewachsen ist, könnte wieder schrumpfen, abhängig von den konkreten Liefermodi und -szenarien. Insbesondere Jobs für gering ausgebildete Menschen könnten wegfallen. Eine massive Umstellung des Systems auf Drohnenlieferungen „auf der letzten Meile“ müsste berücksichtigen, dass Drohnen wegen des Wetters nicht immer fliegen können, also ein redundantes Liefersystem benötigt würde.

Es ist Lärmbelästigung zu erwarten, da zwar die einzelne Drohne leise, aber Schwärme laut wären. Es steht weiters zu erwarten, dass Teile der Bevölkerung mit der massiven Nutzung des bodennahen Luftraums auch aus ästhetischen Gründen nicht einverstanden sein werden.

Um autonom fliegen zu können, wären Drohnen mit einer Vielzahl von Sensoren und Kameras ausgerüstet, die eine große Menge von potenziell sensiblen Daten erzeugen. Diese können gespeichert und missbraucht werden. Drohnen können darüber hinaus auf einfache Weise für verschiedene illegale Zwecke missbraucht werden, vom Schmuggel ${ }^{1}$ bis zu terroristischen Absichten. ${ }^{2}$ Missbrauch ist schwierig unter Kontrolle zu bringen.

\section{Relevanz des Themas für das Parlament und für Österreich}

In Österreich gibt es eine Reihe von Drohnen-Herstellern (z.B. Schiebel, Stromkind, Austrodrones, Dynamic Perspective, Blacksheep etc.) und einige Forschungseinrichtungen, die sich mit Drohnen aus unterschiedlichen Perspektiven beschäftigen (z.B. TU Wien, TU Graz, AEC Linz, AAU Klagenfurt). Amazon testet hierzulande Drohnen und auch die Österreichische Post hat in Graz 2017 einen groß angelegten Pilotversuch durchgeführt.

Das Thema ist seit ca. drei Jahren regelmäßig in den Medien präsent, anfangs hauptsächlich in der Fachpresse, mittlerweile auch in Radio und Fernsehen.

Vor dem Hintergrund einer vorläufigen Abschätzung (ITA 2018) kann von einer Reihe potenzieller Regulierungserfordernisse ausgegangen werden. Es gibt seit mehreren Jahren weltweit verschiedene Gesetzesinitiativen (AAE/3AF 2015, p. 52ff.). Insbesondere müsste, wenn es gesellschaftlich und politisch überhaupt gewollt ist, das Luftverkehrsrecht erst „drohnenfit" gemacht werden; der Bereich KonsumentInnen- und Privatsphärenschutz untersucht werden; eventuell das Steuer- bzw. Abgabenrecht angepasst werden; sowie wirksame Mechanismen zur Rechtsdurchsetzung gefunden werden.

\author{
Arbeitsmarkt und \\ Resilienz des \\ Liefersystems
}

Lärm und
Ästhetik

Privatsphäre und Missbrauch

österreichische Drohnenhersteller, Forschungseinrichtungen und Pilotversuche

\footnotetext{
1 Z.B. washingtontimes.com/news/2017/aug/20/mexican-drug-cartels-usingdrones-to-smuggle-heroi/.

2 Ein aktuelles Beispiel aus den USA: bbc.com/news/technology-44003860.
} 
EU-Verordnung erwartet
Auf EU-Ebene wurde Ende 2017 eine politische Vereinbarung auf Basis von Vorschlägen der European Aviation Safety Agency (EASA) erzielt ${ }^{3}$, womit zu erwarten steht, dass noch 2018 eine EU-Verordnung von der EU-Kommission vorgeschlagen werden wird.

\section{Vorschlag weiteres Vorgehen}

Aufgrund der vielen offenen Fragen und der Konfliktträchtigkeit sollte eine umfassende, auf Österreich fokussierende TA-Studie mit partizipativen Elementen durchgeführt werden. Eine solche interdisziplinär angelegte, ergebnisoffene Studie würde das Wissen von ExpertInnen mit den Interessen der verschiedenen Stakeholder (u.a. Luftfahrtbehörde, Drohnenhersteller, Logistikunternehmen, Polizei) mit den informierten Einschätzungen von Bürgerlnnen, die als Anwohnerlnnen oder Kundlnnen betroffen sein würden, verbinden. Diese Studie würde den bisher einseitig technischen und marktgetriebenen Diskurs auf die gesellschaftliche Ebene heben und eine Entscheidungsfindung unter Einbeziehung aller Sichtweisen vorbereiten. Aufgrund der partizipativen Komponente wäre eine solche Studie etwas größer zu konzipieren.

\section{Zentrale weiterführende Quellen}

AAE/3AF (2015): Present and Future of Civilian Drones. No. AAE Dossier \#40/3AF Cahier \#16, Paris: Air and Space Academy/French Aerospace Society, espas.eu/orbis/sites/default/files/generated/document/en/D40_Ebook_ UK.pdf.

ITA 2018, Delivery drones from a technology assessment perspective. Überblicksbericht. Wien: Institut für Technikfolgen-Abschätzung, epub.oeaw.ac.at/ita/ita-projektberichte/2018-01.pdf.

3 easa.europa.eu/easa-and-you/civil-drones-rpas/drones-regulatory-frameworkbackground; easa.europa.eu/document-library/opinions/opinion-012018. 


\section{Point of Sale 2.0: Automaten der Zukunft}

Ein Verkaufsautomat ist ein Gerät, das gegen Bezahlung Waren ausgibt (Kaugummis, Bücher, Autos, Zigaretten, Kondome, Ab-Hof-Produkte) oder Zugang zu abgesperrten Räumen (Toiletten, Abholstationen) oder Dienstleistungen (Internetzugang, Gewichtsmessung) gewährt und für die Selbstbedienung durch Kundlnnen bestimmt ist. Automaten sind rund um die Uhr in Betrieb und ein Point of Sale (PoS), der Ort an dem die Waren dargeboten, beworben und verkauft werden. Inzwischen machen smarte Technologien eine neue Generation von Automaten möglich, die digital vernetzt sind und die den Zugang zu Produkten virtuell unterstützen. Das bietet einerseits Vorteile durch erleichterte und personalisierte Bedienung, die durch Social Media, VR- und AR-Technologien, sowie Gesichts-, Gesten- oder Stimmerkennung und Künstliche Intelligenz (KI) ermöglicht wird. Zum Beispiel können virtuelle Assistenten (siehe Social Robots) durch den Einkaufsvorgang am Automaten begleiten oder das dargestellte Angebot kann durch Gesichtserkennung je nach Geschlecht oder Alter des Betrachters - wie digitale Werbeplakate - am Automaten angepasst werden. ${ }^{1}$ Andererseits können vernetzte Automaten leichter konfiguriert werden und damit flexibel in unterschiedlichen Bereichen eingesetzt werden, was für Unternehmen bedeutet, dass keine Automaten mehr angeschafft werden müssen, sondern die Automaten-Dienstleistung einfach zugekauft werden kann („Vending as a Service“). Gerade in Zeiten von Corona können Warenautomaten, neben Hauszustellung und Abholservices, dabei helfen den direkten Kontakt mit Mitmenschen beim Einkaufen zu vermeiden. Besonders im Bereich des Handels mit landwirtschaftlichen Produkten, scheint die Covid-19 Krise den Trend zum Lebensmittelautomaten verstärkt zu haben. ${ }^{2} \mathrm{Ob}$ sie zu einem langfristigen Ersatz von menschlicher Arbeitskraft führt, wird kontrovers diskutiert (Prainsack/Buyx 2018). In der Direktvermarktung landwirtschaftlicher Produkte wird argumentiert, dass die Wartung, Bewerbung und Installation von Automaten oft mit nicht zu vernachlässigendem - Personaleinsatz verbunden ist. ${ }^{3}$ Unbestritten ist hingegen die Zunahme an wertvollen Nutzerdaten, die durch den vermehrten Einsatz digitaler Technologien in Automaten generiert werden. Beispielsweise beim kontaktlosen Bezahlvorgang oder wenn anhand von Eye-Tracking und Machine-Learning Technologie das Konsumentenverhalten am Automaten ausgeforscht wird, um das Warenangebot $\mathrm{zu}$ personalisieren und zu verbessern (Pfeiffer et al. 2020). Während im Bereich Fintech bereits europäische Alternativen zu globalen Payment-Anbietern entwickelt werden, um eine sichere und kontaktlose Zahlungsab-

\footnotetext{
1 trendsderzukunft.de/sehender-verkaufs-automat-weis-was-kunden-wollen/.

2 steiermark.orf.at/stories/3056685/.

3 V. a. wenn der Automat einen zusätzlichen Vertriebskanal darstellt, siehe agrarheute.com/management/so-setzen-landwirte-verkaufsautomaten-535051; Ifl.bayern.de/mam/cms07/publikationen/daten/informationen/direktvermarktungautomaten-vertrauenskassen-forum-diversifizierung_Ifl-information.pdf.
} 
wicklung am Automaten zu ermöglichen ${ }^{4}$, bleibt die Frage nach europäischen Plattformen für den personalisierten Automatenverkauf vorerst offen (siehe Dezentralisierte Kollaborationsplattformen).

\section{Zitierte Literatur}

Pfeiffer, J., Pfeiffer, T., Meißner, M. und Weiß, E., 2020, Eye-Tracking-Based Classification of Information Search Behavior Using Machine Learning: Evidence from Experiments in Physical Shops and Virtual Reality Shopping Environments, Inf. Syst. Res. 31(3), 675-691.

Prainsack, B. und Buyx, A., 2018, The value of work: Addressing the future of work through the lens of solidarity, Bioethics 32(9), 585-592, doi.org/10.1111/bioe.12507.

4 derbrutkasten.com/bluecode-und-secure-payment-technologiesmillionenforderung-fur-europa-expansion/. 


\section{Personalisierte Ernährung: Nudges statt Wedges?}

Gesundheitliche Folgen von ungesunder Ernährung nehmen weltweit zu. Personalisierte Ernährung ist ein Ansatz, mit dem versucht wird diesen Risiken entgegenzuwirken. Dazu zählen z. B. funktionale Nahrung, die mit Hilfe biotechnologischer Methoden und Genomanalyse an individuelle Bedürfnisse angepasst wird (siehe Funktionelle Nahrung aus dem Labor), oder auch maßgeschneiderte Ernährungskonzepte, die auf die persönliche Darmbakteriengemeinschaft ${ }^{1}$, das genetische Profil und den Lebensstil abgestimmt werden. ${ }^{2}$ Dabei spielen digitale Technologien eine wichtige Rolle, die die Personalisierung beim Lebensmitteleinkauf oder dem Restaurantbesuch ermöglichen und so eine umfassende Überwachung der eigenen Ernährungsgewohnheiten und Gesundheitsparameter unterstützen. ${ }^{3}$ Darüber hinaus versprechen personalisierte Ansätze zur Förderung der diätischen Gesundheit geringere Gesundheitsausgaben und einen großen Markt für diagnostische Anwendungen (Abrahams et al. 2019). Doch was bringt personalisierte Ernährung? Kann die Gesundheit der Bevölkerung langfristig mit individuell zugeschnittenen Empfehlungen verbessert werden? Werden die Menschen motiviert sein, sich an eine maßgeschneiderte Ernährung zu halten? Zweifel in Hinblick auf Datenschutz, mangelndes Vertrauen in die Gentechnologie und fehlendes Wissen über die Rolle der Genetik bei chronischen Krankheiten verlangsamen die breite Anwendung des personalisierten Ansatzes (Abrahams et al. 2019). Aber auch soziale Situationen, wie z. B. die gemeinsame Mahlzeit in der Familie, können sich zunehmend schwierig gestalten, wenn jede Person ihren eigenen Diätplan einhalten möchte. Die größte Hürde für personalisierte Ernährung ist die Akzeptanz der BürgerInnen.

Ein Versuch, der speziell darauf abzielt individuelle Verhaltensänderungen zu bewirken, ist Nudging (für weitere Information zu Nudging allgemein siehe Digitales Nudging und Demokratie). Im Bereich der Ernährung zählt die Lebensmittelkennzeichnung, Verbesserung der Sichtbarkeit von Produkten (,cognitive nudges“), aber auch Interventionen, die darauf abzielen die Wahrnehmung durch affektive Produktgestaltung zu beeinflussen (,sensorisches Marketing“), zu den gängigen Nudging-Methoden. Ein extremes Beispiel für Nudging im Ernährungsbereich ist die Aktion von mehreren Fast Food Restaurants, die vor ihren Eingängen Stäbe in unterschiedlichen Abständen montiert haben, um Menschen - freiwillig - abhängig von inrem Körperumfang ins Lokal zu lassen und je nachdem, durch welche Öffnung sie kommen (je schmäler der Durchlass, desto höher der Rabatt), den Kundlnnen bis zu 100\% Preisnachlass auf konsumierte Speisen ge-

\footnotetext{
1 microbiomesupport.eu.

2 Forschungsunterfangen in diesen Bereichen werden unter Nutrigenomik gefasst, ein Gebiet, das Erkenntnisse aus Genomforschung, Pflanzenzüchtung, Gentechnik und moderner Medizin verknüpft, um Nahrungsmittel zu entwickeln, die in der medizinischen Prävention und Behandlung eingesetzt werden können.

3 Unzählige Diät-Apps für das Smart Phone zeugen davon.
} 
währen. ${ }^{4}$ Ziel der Aktion ist es It. Restaurantbetreibern, Bewusstsein für Ernährung zu stiften. Ob diese Art personalisierter Ansätze in Zukunft die Akzeptanz der BürgerInnen erhöht und ihre Ernährungsgewohnheiten langfristig verbessert ist jedoch fraglich. Interessant und vielversprechend sind hingegen Studien, die darauf abzielen herauszufinden, welche $\mathrm{Be}-$ dingungen das Konzentrationsvermögen und das bewusste Denken bei Entscheidungen fördern und so die Wahrscheinlichkeit von Fehlentscheidungen verringern (Rebonato 2014). Das Zukunftspotenzial solcher kontrastierenden Ansätze zur Verhaltensänderung - ganz im Sinne von „Teaching“ statt „Nudging“ - könnte weiter ausgelotet werden.

\section{Zitierte Literatur}

Abrahams, M., Frewer, L. J., Bryant, E. und Stewart-Knox, B., 2019,

Personalised Nutrition Technologies and Innovations: A Cross-National Survey of Registered Dietitians, Public Health Genomics 22(3-4), 119-131, karger.com/DOI/10.1159/000502915.

Rebonato, R., 2014, A Critical Assessment of Libertarian Paternalism, Journal of Consumer Policy 37(3), 357-396, doi.org/10.1007/s10603-014-9265-1.

4 dailymail.co.uk/news/china/article-5708899/Restaurant-offers-discounts-skinnycustomers-asking-squeeze-metal-bars.html; thesmartlocal.com/thailand/easyray-challenge/. 


\section{Sensorrevolution: Smarte Städte - smarte Menschen?}

Die Sensorrevolution zielt auf die Verbindung von Computern zur realen Welt ab und verspricht nahezu unbegrenzte Anwendungsmöglichkeiten. Sensortechnologien reichen von lokalen und mobilen Luftverschmutzungsmessgeräten, über Fingerabdrucksensoren oder 3D-Sensoren zur Gesichtserkennung; von tragbaren Fitness-Messgeräten zur Erfassung personalisierter Gesundheitsdaten, bis hin zur Vision unzähliger Minisensoren, die als eine Art globales Nervensystem („Intelligenter Staub“) überall auf der Erde Daten sammeln und in Verbindung mit leistungsfähigen Computernetzwerken neue Erkenntnisse bringen sollen (Lohr 2010). Die Verbindung von Sensorik mit neuen Mobilfunktechnologien, wie 5G Internet (siehe Thema „5G“), könnte zukünftig die Effizienz und Flexibilität von vernetzten Sensorsystemen weiter steigern und eine regelrechte „Sensorrevolution" auslösen.

Die effiziente und detailgenaue Erfassung und Vernetzung von Umweltsowie Personendaten durch intelligente Sensoren verspricht einerseits eine erhöhte Problemlösungskapazität, wenn es beispielsweise um das Monitoring von Umweltverschmutzung (Daten von Smart Watches zur Erstellung von interaktiven Luftverschmutzungskarten), die Automatisierung von Fertigungsprozessen durch Vernetzung von Maschinen- und Gerätedaten (Industrie 4.0), oder die Regulierung von Smarten Gebäuden geht (Thermostate zur Steuerung der Wohnungstemperatur über internetfähige Mobilgeräte, siehe Thema "++-Energiegebäude“). Bei der Erfassung von Umweltdaten verlässt sich die Wissenschaft oftmals auf Bürgerlnnen („Citizen Sensing “1 ${ }^{\prime 1}$, die ohnehin täglich ihre mobilen und Sensor-bepackten Geräte herumtragen.

Andererseits verweist die aufkommende Praxis der Selbstoptimierung auf die möglichen Schattenseiten der bevorstehenden Sensorrevolution: ständige Messung von Schlafphasen, Ernährungsverhalten und Produktivität durch Anwendungen, die versprechen beim Abnehmen zu helfen, die Leistungsfähigkeit zu steigern oder die Gesundheit zu fördern. Der EchtzeitAustausch von Sensordaten ermöglicht den umfassenden Abgleich individueller Daten mit anderen Internet-NutzerInnen, was z. B. im Fall von persönlichem Fitness-Tracking oder Leistungs-Monitoring zu erhöhtem sozialen Druck und zur übertriebenen Selbstausbeutung führen könnte (Friedrichs 2013; ITA 2018). Sensortechnologien in Verbindung mit neuen Innovationen wie Exoskeletten (siehe Thema „Exoskelette“), die eine umfassende Überwachung von ArbeitnehmerInnen ermöglichen und/oder erfordern, verstärken die Bedenken um Persönlichkeitsrechte und -schutz.

Als Vorbereitung auf eine bevorstehende Sensorrevolution, wäre es sinnvoll zu klären, wie Sensoren die Selbst- und Umweltwahrnehmung verändern und dadurch neue Normvorstellungen prägen, welche Bildungsmaßnahmen die sinnvolle Integration von Sensortechnologien ins Altagsleben

1 citizensense.net. 
ermöglichen und wie Persönlichkeitsrechte und Privatsphäre in einer vernetzten Welt garantiert werden können.

\section{Zitierte Literatur}

Friedrichs, J., 2013, Selbstoptimierung. Das tollere Ich., Zeit Online.

ITA (Hrsg.), 2018, Selbst vermessen - fremd gesteuert. ITA-Dossier Nr. 35

(April), epub.oeaw.ac.at/ita/ita-dossiers/ita-dossier035.pdf.

Lohr, S., 2010, Smart Dust. Not Quite, but We're Getting There, The New

York Times. 


\section{Virtuelle und augmentierte Realitäten}

\section{Zusammenfassung}

Technologien zur Erzeugung virtueller und augmentierter Realitäten befinden sich momentan in einer Aufschwungphase. Die großen und namhaften Technologieunternehmen (Microsoft, Apple, Google, Facebook, Amazon) versuchen sich in diesem neuen Technologiesektor zu positionieren. Die Anwendungsgebiete sind sehr breit gefächert und umfassen nahezu jeden wirtschaftlichen Bereich. Erste Anwendungsbeispiele gewähren bereits jetzt einen Einblick in die Potentiale dieser neuen Technologie. Zugleich jedoch wirft ihre Anwendung neue Herausforderungen und Probleme auf. Bei Augmented Reality (AR) stellt sich beispielsweise die Frage, unter welchen Bedingungen und ob überhaupt digitaler öffentlichen Raum für alle möglichen Anwendungen genutzt werden soll (Unterhaltung, Werbung, Kunst etc.). Bei Virtual-Reality-Anwendungen (VR) stellt sich die Frage, welche Auswirkungen die Technologie auf die NutzerInnen und deren Psyche und Selbstwahrnehmung hat. Erste Studien deuten darauf hin, dass Auswirkungen auf die Psyche und Selbstwahrnehmung der NutzerInnen bestehen. Hierbei spielt die Immersion (Eintauchen), also der Grad, inwieweit die virtuelle Realität von der menschlichen Sensorik als real empfunden wird, eine wesentliche Rolle.

Mit der zunehmenden Verbreitung dieser Technologien, allen voran über Smartphones, können diese und zahlreiche weitere Fragen bereits in naher Zukunft sowohl aus innovations- und wirtschaftspolitischer als auch aus gesundheits- und konsumentenschutzpolitischer Perspektive relevant werden.

\section{Überblick zum Thema}

Virtuelle Realitäten (VR) bezeichnen künstliche erstellte (virtuelle) Umgebungen, die der Wahrnehmung des Menschen über Sehen und Hören durch geeignete Technologien zugänglich gemacht werden. Man unterscheidet zudem virtuelle Realitäten, in denen eine gänzlich künstliche Realität erzeugt wird, von so genannten erweiterten (extended) oder augmentierten Realitäten (AR), welche durch eine Überlagerung aus der tatsächlichen Realität mit einer künstlich erstellten, digitalen Realität erzeugt wird. Die eigesetzten Technologien reichen von so genannten „Head Mounted Displays", also Bildschirmen, die aufgesetzt werden (z. B. Oculus Rift, HTC Vive, PS-VR), „Smartglasses“" (z. B. google glass), über unterschiedliche Formen von Head-Up-Displays bis hin zu Handheld-Geräten wie Smartphones (Palmarini et al. 2018; Rese et al. 2017).

Gerade Smartphones bieten sich mit ihrer reichhaltigen Grundausstattung an verschiedenen Sensoren (Beschleunigung, Barometer, GPS, Gyroskop, Kompass u.v.m.) zur weiteren Verbreitung der AR- und VR-Technologien an. Die Einführung von Augmented Reality Software Development Kits von Google (ARCore) und Apple (AR-Kit) Mitte 2017 lassen darauf schlie- 


\section{breite \\ Anwendungsfelder denkbar}

Unfallgefahr

Vandalismus ßen, dass dieser Technologiebereich in den nächsten Jahren zunehmend wachsen und an Bedeutung gewinnen wird. Dieser Trend führt dazu, dass AR- und VR-Technologien einem Großteil der Gesellschaft relativ rasch zugänglich gemacht werden (durch die hohe Verbreitung von HandheldGeräten). Schätzungen gehen davon aus, dass das Marktpotential von VR und AR im Jahr 2021 bei umgerechneten 92 Mrd. $€$ liegen wird, wobei die mobile Anwendung von AR mit umgerechnet 70 Mrd. $€$ den Löwenanteil davon ausmachen wird. ${ }^{1}$ Optimistische Schätzungen gehen von Impulsen für die Weltwirtschaft in Höhe von 1,6 Billionen Euro im Zusammenhang mit VR/ AR bis 2030 aus. $^{2}$

Vor diesem Hintergrund haben zurzeit nahezu alle großen Unternehmen Pläne, sich in diesem Bereich zu betätigen. ${ }^{3}$ Die Anwendungsgebiete sind breit: im Bereich der Industrie 4.0, um zum Beispiel Menschen bei Wartungsarbeiten zu instruieren ${ }^{4}$; in der Medizin (z. B. Chirurgie) $)^{5}$; in Schulen für interaktives Lernen6; oder in der Unterhaltungsindustrie, um Film- oder Spieleerlebnisse noch intensiver wirken zu lassen. ${ }^{7}$ Auch für die Werbeindustrie bietet die Technologie viele neue Möglichkeiten, wie das Beispiel eines „The North Face“-Shops in Südkorea zeigt: Kundlnnen konnten, nachdem sie eine neue Jacke anprobierten, eine virtuelle Hundeschlittenfahrt absolvieren, was ihr Einkaufserlebnis verstärken sollte. ${ }^{8}$

Neben dieser Fülle an Möglichkeiten, die die Technologie mit sich bringt, lassen sich schon potentielle Risiken bzw. potentielle Handlungsfelder identifizieren. Pokemon Go als eine der ersten breiten AR-Anwendungen, hat alleine in den ersten zehn Tagen zu 110.000 Verkehrsunfällen geführt (durch die Unaufmerksamkeit von FahrerInnen oder PassantInnen). Es wurden sogar zahlreiche Todesfälle registriert, die im Zusammenhang mit Pokemon Go stehen. ${ }^{9}$ Im Oktober 2017 hat eine neue Zusatzfunktion in der populären Social-Media-Plattform Snapchat für Aufsehen gesorgt: In Zusammenarbeit mit dem Künstler Jeff Koons wurde ein Feature zur Ausstellung von AR-Kunst entwickelt. Hierbei wurde eines seiner Exponate (Balloon Dog) im Central Park als AR-Kunst dargestellt und man konnte es mit dem Smartphone und entsprechender App betrachten. Der Künstler Sebastien Errazuriz hat Jeff Koons digitales Exponat virtuell vandalisiert, um auf die Problematik der Nutzungsrechte von digitalen öffentlichen Räu-

\footnotetext{
1 techcrunch.com/2017/01/11/the-reality-of-vrar-growth/.

2 pwc.com/seeingisbelieving.

3 goldmansachs.com/our-thinking/pages/technology-driving-innovationfolder/virtual-and-augmented-reality/report.pdf.

4 youtube.com/watch?v=SfG33CNqq-w.

5 bdc.de/augmented-reality-in-der-chirurgie-wie-wird-unsere-wahrnehmungerweitert/.

6 zspace.com.

7 thenextweb.com/augmented-reality/2017/09/13/the-machines-is-the-first-gottahave-it-title-for-ar-since-pokemon-go/.

8 adweek.com/creativity/north-face-gave-these-shoppers-vr-experience-suddenlygot-awesomely-real-167900/.

9 pokemongodeathtracker.com.
} 
men aufmerksam zu machen. ${ }^{10}$ Im Kontext der steigenden Popularität und Anwendung von AR-Technologien, stellt sich weiters die Frage, ob Augmented-Reality-Erlebnisse nach ähnlichen Regeln wie die Vermietung physischer öffentlicher Räume geregelt werden sollten. Inwieweit sollen Unternehmen das Recht bekommen, GPS-Daten von öffentlichen Räumen für kommerzielle Zwecke zu nutzen, vor allem, wenn es sich um Werbung handelt? Es zeichnet sich bereits ab, dass das Technologiefeld rund um AR und VR neben seinen hohen marktwirtschaftlichen Potentialen auch nicht zu vernachlässigende Folgen mit sich bringen wird. Die (noch) bestehende Möglichkeit zur Gestaltung der sozio-technischen Innovationspfade rund um AR und VR in Österreich sollte demnach früh ergriffen werden.

\section{Relevanz des Themas für das Parlament und für Österreich}

Die breiten, vielversprechenden Anwendungsfelder virtueller und augmentierter Realitäten machen das Thema auch für das österreichische Parlament relevant. Aus Sicht der Innovations- und Wirtschaftspolitik ergibt sich die Relevanz dadurch, dass die Entwicklung des Technologiesektors VR und AR über zielgerichtete Technologieförderung erfolgen kann, zumal abzusehen ist, dass AR insbesondere im Bereich von Industrie 4.0 eine wesentliche Rolle spielen wird. Die Existenz der Virtual and Augmented Reality Association Austria (VARAA) ${ }^{11}$ als Fachverband professioneller VR/ AR-AnwenderInnen und Unternehmen in Österreich deutet darauf hin, dass in Österreich bereits eine gewisse Dynamik besteht.

Mit der zunehmenden Bedeutung dieses Technologiesektors geht auch der Bedarf einer systematischen Auseinandersetzung aus Sicht des KonsumentInnenschutzes einher. Aktuelle Studien deuten unter anderem auf potentielle Auswirkungen auf die Psyche bzw. die Selbstwahrnehmung der NutzerInnen hin (Madary/Metzinger 2016, Jimenez et al. 2020). Auch Soziale Medien-Plattformen werden in Zukunft AR/VR Technologien verwenden, was zu einer Verstärkung negativer Folgen von deren Nutzung führen kann: Depressionen, Gefühle der Isolation und Einsamkeit, negative Körperbilder, Egozentrik und Narzissmus (Studen \&Tiberius 2020). Damit ergibt sich eine unmittelbare Relevanz für Gesundheitspolitik und KonsumentInnenschutz. Die zuvor genannte Problematik des digitalen öffentlichen Raums und der Nutzungsrechte daran wirft zudem neuartige Fragen auf, die zeitgerecht im öffentlichen Interesse geklärt werden müssen. Eine gemeinsame Betrachtung dieser Aspekte scheint sinnvoll, da das Voranschreiten der Technologien in vielen unterschiedlichen Anwendungsfeldern parallel, aber dennoch interdependent verläuft und nicht auf einen einzelnen Bereich festgelegt werden kann.
Technologieförderung

\section{Schutz der \\ KonsumentInnen}

Nutzungsrechte

\footnotetext{
10 nytimes.com/2017/10/10/arts/design/augmented-reality-jeff-koons.html.

11 varaa.at.
} 


\section{Vorschlag weiteres Vorgehen}

Eine mögliche Herangehensweise im Zuge einer Langstudie wäre es, zunächst einen breiten systematisierten Überblick zu den aktuellen technologischen Fortschritten und Anwendungsgebieten von augmentierten und virtuellen Realitäten zu erstellen. In einem nächsten Schritt könnten unter Einbindung nationaler Stakeholder (z. B. Virtual and Augmented Reality Association Austria, Technologieentwickler) Kompetenzfelder für die österreichische F\&E-Landschaft identifiziert werden. Ziel wäre es, international kompetitiv agieren zu können und das in Österreich bestehende Innovationspotential bestmöglich auszuschöpfen. Hierbei muss aber besonderes Augenmerk auf die bereits oben angedeuteten und sich abzeichnenden Technikfolgen gelegt werden. Der gegenwärtige Technologiestatus bietet noch ausreichend gesellschafts- und wirtschaftspolitischen Gestaltungsspielraum. Die Studie soll dazu dienen, möglichen Handlungsbedarf zu identifizieren und diesen operationalisierbar zu machen.

\section{Zentrale weiterführende Quellen}

Jimenez D.E., Shah J., Das P., Milanaik R.L. (2019) Health Implications of Augmented Reality Games on Children and Adolescents. In: Geroimenko V. (eds) Augmented Reality Games I. Springer, Cham. doi.org/10.1007/978-3-030-15616-9_10.

Madary, M. und Metzinger, T. K., 2016, Real Virtuality: A Code of Ethical Conduct. Recommendations for Good Scientific Practice and the Consumers of VR-Technology, Frontiers in Robotics and Al 3(3), frontiersin.org/article/10.3389/frobt.2016.00003.

Palmarini, R., Erkoyuncu, J. A., Roy, R. und Torabmostaedi, H., 2018, A systematic review of augmented reality applications in maintenance, Robotics and Computer-Integrated Manufacturing 49(Supplement C), 215-228, sciencedirect.com/science/article/pii/S0736584517300686.

Rese, A., Baier, D., Geyer-Schulz, A. und Schreiber, S., 2017, How augmented reality apps are accepted by consumers: A comparative analysis using scales and opinions, Technological Forecasting and Social Change 124(Supplement C), 306-319, sciencedirect.com/science/article/pii/S0040162516304528.

Studen, L.; Tiberius, V. Social Media, Quo Vadis? Prospective Development and Implications. Future Internet 2020, 12, 146. doi.org/10.3390/fi12090146. 


\section{Fliegende Windenergie}

Eine neue Generation Turbinen könnte den Windenergiesektor revolutionieren. Derzeit sind mehrere Prototypen im Einsatz, an denen geforscht wird: Mit dem Boden verbundene Flugdrachen fliegen dabei in einer Höhe von etwa 200-600 m und nutzen den dort herrschenden stetigen und kräftigen Luftstrom. Äußerlich erinnern sie an Segelflugzeuge, Drohnen, Zeppeline oder auch Flugzeugturbinen. ${ }^{1}$ Ihre Entwicklung wurde erstmals während der Energiekrise in den 1970er Jahren angeregt (Zillmann/Bechtle 2018). Strom produzieren diese Windenergiesysteme durch Rotoren auf oder in dem Drachen. Die Energie wird durch ein Kabel zum Boden geleitet. $^{2}$ Ein anderes Design ist mit einer Turbine kombiniert, die am Boden steht. Der Drachen übt dabei ständig Zug auf das Verbindungsseil aus, dieser Zug wird in der Turbine in Strom umgewandelt. Autonom gesteuerte Flugmanöver in Form einer großen Acht mit mehreren hundert Metern Durchmesser erhöhen den Zug, beziehungsweise verstärken die Windgeschwindigkeit an den Rotoren. Vorläufige Ergebnisse deuten auf eine höhere Energieproduktion und Effizienz als herkömmliche Windräder hin. ${ }^{3}$

Große Energieunternehmen wie e.on experimentieren bereits mit der neuen Technologie und auch Google ist in den Markt durch den Aufkauf von kleinen Entwicklern eingestiegen, in die auch Shell investiert. Als potentielles Hauptanwendungsgebiet scheinen sich Offshoreanlagen abzuzeichnen. Allerdings befinden sich die fortschrittlichsten Unternehmen mit ihren Prototypen noch in der Proof-of-Concept-Phase und eine breite Kommerzialisierung ist in naher Zukunft nicht zu erwarten (Hussen et al. 2018). Das erste deutsche fliegende Windkraftwerk ging als Pilotprojekt 2020 in den Dauerbetrieb, nachdem die Anlage mit einem $120 \mathrm{~m}^{2}$ großen Drachen eine luftfahrtrechtliche Evaluierung bestand. ${ }^{4}$ Die Anwendung in Österreich ist potentiell denkbar und könnte einen Übergang zu erneuerbaren Energien unterstützen und beschleunigen. Aufgrund der Kollisions- und Absturzgefahr eignen sich die fliegenden Turbinen vor allem für unbewohnte Gebiete. Auch in bewohnten Gebieten könnten die neuen Windenergiesysteme bei ausreichendem Sicherheitsabstand eingesetzt werden, allerdings sind dabei Standortfragen vor allem auch mit der Bevölkerung abzustimmen. Zuerst müssen sich die neuen Systeme aber im Dauerbetrieb beweisen (Hussen et al. 2018).

\footnotetext{
1 altaerosenergies.com; x.company/makani/.

2 greentechmedia.com/articles/read/a-beginners-guide-to-the-airborne-windturbine-market\#gs.F8LhaSI.

3 global.handelsblatt.com/companies-markets/e-on-invests-millions-in-flying-windturbines-746616.

4 www.skypower100.de/english/news/wind-power-of-the-future/.
} 


\section{Zitierte Literatur}

Hussen, K. v., Dietrich, E., Smeltink, J., Berentsen, K., Sleen, M. v. d., Haffner, R. und Fagiano, L., 2018, Study on Challenges in the commercialisation of airborne wind energy systems, Luxembourg: European Commission - Directorate-General for Research and Innovation, publications.europa.eu/en/publication-detail/-/publication/a874f843c137-11e8-9893-01aa75ed71a1/language-en/format-PDF/source76863616

Zillmann, U. und Bechtle, P., 2018, Emergence and economic dimension of airbornewind energy, in: Schmehl, R. (Hg.): Airborne Wind Energy: Springer Nature. 


\section{Wasserstoffspeicher der Zukunft}

Wasserstoff ist ein regenerativer und umweltfreundlicher Energieträger mit dem höchsten Brennwert aller chemischen Brennstoffe - bezogen auf die Masse (Züttel 2004). Die zwei wesentlichen Gründe, warum Wasserstoff noch nicht umfassend als Brennstoff genutzt wird sind erstens, dass Wasserstoff in der Natur fast nur in gebundener Form als Wasser und Kohlenwasserstoff vorkommt und daher erst produziert werden muss und zweitens ist Wasserstoff bei Raumtemperatur gasförmig und lässt sich aufgrund seiner niedrigen kritischen Temperatur nur schwer speichern.

In der Forschung gibt es unterschiedliche Methoden und technologische Ansätze, um Wasserstoff zu speichern. Dazu zählen gasförmige Speicherung in Hochdrucktanks, kryogene Speicherung von flüssigem Wasserstoff und die Feststoffspeicherung. Die derzeit gängigste Methode ist die Speicherung in Hochdrucktanks, die Wasserstoffgas bis auf 700 Bar zusammenpressen und somit ca. fünf Kilogramm Wasserstoff aufnehmen können (Schröder 2009). Das Problem dabei ist die unhandliche Größe von zwei voluminösen Koffern, die ein solches Hochdrucktanksystem beansprucht.

Eine andere Möglichkeit ist die Speicherung von Wasserstoff durch Einlagerung in metall-organische Gerüststrukturen (Metal-Organic-Frameworks, MOFs). MOFs sind leicht wie Styroporkügelchen und können derzeit aufgrund ihrer porösen Kristallgitter mit großer Oberfläche zwischen fünf und sieben Gewichtsprozent Wasserstoff speichern (Schröder 2009). Für den Einsatz in einem Auto sollten die MOFs mindestens neun Gewichtsprozent Wasserstoff speichern können. Eine weitere Hürde für die Praxis sind die benötigten tiefen Temperaturen bei der Einlagerung (minus $196^{\circ} \mathrm{C}$ ).

Neben MOFs wird auch an anderen Speichersubstanzen geforscht: z. B. an leichten Metallhydriden oder biomimetischen Kohlenstoffgerüsten. Leichte Methallhydride sind bereits als Speichermaterialien im Einsatz, bspw. auf modernen U-Booten. Für die Speicherung von fünf Kilogramm Wasserstoff werden $250 \mathrm{~kg}$ Metallhydrid benötigt (Schröder 2009). Das ist zwar als Zusatzgewicht für den Tauchgang vorteilhaft, für den Einsatz in der Automobilindustrie jedoch ungeeignet. Außerdem verläuft die Einlagerung von Wasserstoff in Metallhydriden äußerst langsam. Schnellere Ladezyklen versprechen die porösen Kohlenstoffgerüste, die wie eine Lunge funktionieren: durch große Öffnungen dringt das Gas tief ins Material, wo es dann wie in den Bronchien in immer feinere Verästelungen gelangt (Schröder 2009).

Die sichere Speicherung von großen Mengen Wasserstoff ist für die erfolgreiche Nutzung von Wasserstoff als Energieträger fundamental. Ausgereift ist bisher keine der beschriebenen Technologien. Trotzdem ist das internationale Interesse von Industrie und Regierungen an Speicherlösun- 
gen groß. Mit maßgeblichen Forschungseinrichtungen ${ }^{1}$ und innovativen Unternehmen verfügt Österreich über adäquate Bedingungen für erfolgversprechende Forschung im Bereich der Wasserstoffspeicherung.

\section{Zitierte Quellen}

Schröder, T., 2009, Wasserstoffspeicher. Das Raumwunder im Tank; MaxPlanck-Gesellschaft; mpg.de/1326157/wasserstoff.

Züttel, A., 2004, Hydrogen storage methods, Naturwissenschaften 91(4), $157-172$

1 hycenta.at. 


\section{Autonome Mini-Häuser}

Immer mehr Firmen produzieren Klein-Häuser die von jeglicher Infrastruktur unabhängig sind. Diese Häuser, meist 20-30 $\mathrm{m}^{2}$ klein, stehen in der Tradition klassischer Wohnwagen, haben aber alle Installationen, die auch in einem normalen Haus zu finden sind. ${ }^{1}$ Viele Anbieter werben mit dem Komfort eines hochpreisigen Hotelzimmers. Solarzellen und Wasseraufbereitung machen diese Häuser autark und, da sie oft auch Räder haben, sind sie weitgehend ortsunabhängig. Steigende Wohnraumpreise in Städten und Umland, wie auch wiederauflebender Minimalismus als Designleitlinie und im Lebensstil führen zu einem wachsenden Angebot autarker Minihäuser, deren Aufstellung auch nicht immer an Bauland gebunden ist, sondern auch in Kleingärten oder unter Umständen auch auf Grünland stehen können. Ohne durch traditionelle städtische Dienstleistungen, wie Strom- und Wassernetz eingeschränkt zu werden, könnten neue Häuser in Gebieten jenseits der städtischen Planungssteuerung entstehen. Eine solche Zersiedelung kann erhebliche ökologische und soziale Folgen nach sich ziehen. So ist beispielsweise eine an Zersiedelung gekoppelte Erhöhung des Individualverkehrs mit mehr Umweltbelastung, aber auch längeren Pendelzeiten verknüpft. Diese Eigenständigkeit der Mini-Häuser führt zu weniger Abstimmungsbedarf im Energiesystem und steht einem anderen Trend entgegen, in dem Gebäude zwar autonomer in ihrer Energieproduktion werden, aber als Produzenten und Verbraucher am gemeinsamen Energienetz teilnehmen und dadurch erheblicher Abstimmungsbedarf entsteht. Wurden früher mobile Heime vornehmlich für Urlaub oder Ferien benutzt, könnte ihre Verbreitung als Hauptwohnsitz längerfristig auch den heute vorherrschenden Wohnstandard senken. Insgesamt ergeben sich damit auch für Österreich relevante Fragestellungen zur Planung und Steuerung der Raumentwicklung und der Energienetze.

1 wohnwagon.at; ecocapsule.sk; passivdom.com/en/; cahute.eu/?lang=en. 


\section{Zukunft der Mensch-Maschine-Interaktion: Spracherkennung und -steuerung}

Die Sprache ist eine zentrale Kulturtechnik und deren Rolle in der Entwicklung der menschlichen Zivilisation ist unumstritten. Gegenwärtig zeichnet sich der Trend ab, dass der Sprache eine immer größere Bedeutung in der Mensch-Maschine-Interaktion zukommt, da die Entwicklungen von Hard- und Software in den letzten Jahren große Fortschritte im Bereich der Spracherkennung gebracht haben. Diese Fortschritte ermöglichen ein breites Anwendungsspektrum für neue Interaktionsarten von Mensch und Maschine. So ist es beispielweise den NutzerInnen von Smartphones bereits möglich, mittels der im Betriebssystem integrierten Sprache-zu-TextAnwendungen, Nachrichten zu verfassen. Entsprechend besteht zudem die technische Möglichkeit, das Gesprochene in Echtzeit in andere Sprachen zu übersetzen. Weitere Anwendungsmöglichkeiten ergeben sich für Menschen mit eingeschränkter Mobilität. In diesem Anwendungsfeld spielt neben der Spracherkennung vor allem auch die Sprachsteuerung eine wesentliche Rolle. Für die NutzerInnen von Spracherkennungsanwendungen bedeutet das einerseits den Zugewinn an Komfort, da eine manuelle Interaktion mit der Maschine - sei es nun ein Smartphone, Tablet oder PC nicht mehr notwendig ist, und andererseits einen Zugewinn an Autonomie.

Alle großen Technologieanbieter sind an der Weiterentwicklung von Spracherkennung und -steuerung beteiligt und bieten diese auch als eigene Geräte zum Verkauf an (z. B. Amazon Echo, Google Home etc.). Diese Geräte sind in die digitalen "Ökosysteme" der jeweiligen Hersteller eingebettet, was vor allem aus Sicht der Datensouveränität und des Datenschutzes Fragen aufwirft. Außerdem haben viele Systeme technische Sicherheitslücken (Edu et al. 2020).

Ein Whitepaper von Microsoft aus dem Jahr 2019 über Sprachtechnologie und digitale Assistenten widmet sich der Frage der Akzeptanz und den zukünftigen Potentialen dieser Technologie. Aus Sicht von Microsoft wird die „Touchscreen-Gesellschaft“ über kurz oder lang von der „Voice-Assistant-Gesellschaft" abgelöst werden. Der Bericht umfasst auch die Ergebnisse einer Onlineumfrage. Sie zeigen, dass etwas über $40 \%$ der Befragten dieser Technologie in Bezug auf ihre Privatsphäre kritisch gegenüberstehen. Die größten Sorgen wurden im Hinblick auf die Sicherheit der personenbezogenen Daten bzw. auf die potentielle Gefahr des latenten Mithörens durch permanent aktive Mikrofone geäußert. ${ }^{1}$ Im April 2019 hat Bloomberg berichtet, dass Amazon die Aufnahmen und Befehle der Sprachassistenten durch Mitarbeiterlnnen anhören, transkribieren und überprüfen lässt. Das Ziel davon ist es, die Spracherkennung zu verbes-

1 advertiseonbing-blob.azureedge.net/blob/bingads/media/insight/whitepapers/ 2019/04\%20apr/voice-report/bingads_2019voicereport.pdf. 
sern. ${ }^{2}$ Diese Praktiken werfen zahlreiche datenschutzrechtliche Fragen auf. Datenschutzfreundliche Einstellungen bieten derzeit keine der Anbieter an, es werden detaillierte Persönlichkeitsprofile erstellt und direkt mit konkretem Verhalten der AnwenderInnen verknüpft (Schaber et al. 2019). Der Zugewinn an Komfort geht somit mit einer Einbuße an Privatsphäre einher (Ferdinand/Jetzke 2017). Die zunehmende digitale Durchdringung der Gesellschaft durch diese Technologie und die damit einhergehenden Fragen sind bislang noch nicht ausreichend geklärt.

\section{Zitierte Literatur}

Jan-Peter Ferdinand, Tobias Jetzke (2017) Voice Computing-allgegenwärtige Spracherkennung. Themenkurzprofil Nr. 15. Büro für TechnikfolgenAbschätzung beim Deutschen Bundestag (TAB), tab-beimbundestag.de/de/pdf/publikationen/themenprofile/Themenkurzprofil015.pdf

Jide S. Edu, Jose M. Such, Guillermo Suarez-Tangil (2020) Smart Home Personal Assistants: A Security and Privacy Review. Preprint: Accepted for publication in ACM Computing Surveys arXiv:1903.05593v3.

Schaber, F., Krieger-Lamina, J., \& Peissl, W. (2019). Digitale Assistenten Endbericht (p. 77). Hrsg. Institut für Technikfolgenabschätzung (ITA) der Österreichischen Akademie der Wissenschaften, Wien. doi:/10.1553/ITA-pb-2019-01.

2 bloomberg.com/news/articles/2019-04-10/is-anyone-listening-to-you-on-alexa-aglobal-team-reviews-audio. 


\section{Roboterautos}

Lange Zeit galt die Fahrerin bzw. der Fahrer als unverzichtbar für alle Fahrzeugtypen. Während ein Autopilot, der lange monotone Abschnitte ohne Hindernisse bewältigen kann, als Standard für Wasser- und Luftfahrzeuge gilt, ist der Sachverhalt für Landfahrzeuge wesentlich schwieriger (siehe auch Autonomer ÖPNV). Die gängige Skala der Automatisierungsstufen geht von null (keine Automatisierung, Fahrerlnnen steuern ohne Hilfen) bis fünf (vollständige Automatisierung, die Maschine steuert ohne Einfluss der InsassInnen), doch in der Praxis wird fast nur an den Stufen bis zwei und an Stufe fünf gearbeitet, während die „Halb-autonomen“ Fahrzeuge der Stufen drei und vier langfristig kaum Beachtung bekommen werden. Dafür dürfte sich die ständige Bereitschaft von FahrerInnen zur rechtzeitigen Rückübernahme der Verantwortung als zu unrealistisches Szenario erweisen. ${ }^{1}$

Die Teilautomation der Stufe zwei ist mittels umfangreicher Assistenzsysteme (Bremsen, Spurhalten, Einparken etc.) bereits Realität. Diese Systeme werden auch immer weiter verbessert. Vollständig autonome Fahrzeuge der Stufe fünf jedoch sind derzeit maximal als Pilotprojekte auf öffentlichen Straßen unterwegs, doch mittlerweile ist eine Welt vorstellbar, in der Menschen nicht mehr obligatorisch Fahrerlnnen eines Fahrzeugs, sondern lediglich Passagiere sind. Auch die österreichische Politik sieht dies als ein Ziel. ${ }^{2}$

Die notwendigen Technologien sind heute noch nicht vollständig ausgereift. Die Aufgabe ist sehr herausfordernd, geht es doch darum, sicher und effizient in allen erdenklichen Situationen in einem sehr komplexen, teils chaotischen Umfeld autonom zu agieren. Typische Verkehrssituationen insbesondere im urbanen Bereich beinhalten auch unvorhersehbar agierende menschliche FahrerInnen in anderen Fahrzeugen und andere VerkehrsteilnehmerInnen. Vor allem die Verlässlichkeit der Sensoren und Algorithmen ist derzeit der Schwerpunkt der F\&E. Der aktuelle Fokus liegt auf der Ausstattung selbstfahrender Fahrzeuge mit wenigen und billigeren Sensoren und auf der Lernfähigkeit der Algorithmen. Jedes große Unternehmen, das an Fahrzeugentwicklungen beteiligt ist, investiert erhebliche Ressourcen, um diese Technologie komfortabel auf den Markt zu bringen.

Abgesehen von den zu erwartenden technologischen Fortschritten in naher Zukunft, stellen sich bereits jetzt eine Reihe von Fragen, auch wenn der öffentliche Einsatz der Technologie nicht so plötzlich erfolgen wird, wie ursprünglich angenommen (vgl. schon Maurer et al. 2015). Allgemein wird

1 adac.de/rund-ums-fahrzeug/autonomes-fahren/recht/autonomes-fahreninterview-armin-mueller/.

2 Siehe den Aktionsplan Automatisierte Mobilität 2019-2022, bmvit.gv.at/service/publikationen/verkehr/automatisiert/downloads/automatisiert 2019_ua.pdf. 
zwar davon ausgegangen (ibid.), ${ }^{3}$ dass Roboterautos am Ende der Entwicklung viel sicherer als von Menschen gelenkte sein werden, für eine vermutlich lange Übergangszeit werden jedoch beide Fahrzeugtypen im Mischverkehr unterwegs sein, was das Gesamtsystem vorübergehend sogar weniger sicher machen könnte. Darüber hinaus sind die elektronischen Systeme an Bord ein potenziell attraktives Ziel für Hackerangriffe. Lernalgorithmen können selbst von ihren Designern nicht vollständig verstanden werden, manche halten sie für prinzipiell unvorhersehbar; es stellt sich die Frage, wie die Gesellschaft mit dieser prinzipiellen Unbeherrschbarkeit umgehen möchte. Weiters wird diskutiert, wie selbstfahrende Fahrzeuge mit ethischen Dilemmata in Gefahrensituationen umgehen würden, denn Unfälle sind nie auszuschließen. ${ }^{4}$ Roboterautos produzieren ständig eine große Menge, teils personenbezogener Daten (insb. Standortdaten), womit sich Fragen des Schutzes der Daten und der Privatsphäre der Passagiere stellen (Krieger-Lamina 2016). Der Einfluss der Entwicklung von Roboterautos auf den Arbeitsmarkt ist nicht zu unterschätzen, denn im Transportsektor und in der Personenbeförderung auf der Straße sind sehr viele Menschen als FahrerInnen beschäftigt. Aufgrund der Kopplung der Transportbranche mit vielen anderen Branchen ist mit Folgewirkungen in ebendiesen zu rechnen. Schließlich stellt sich die Frage, wer die notwendige Infrastruktur aufbaut und finanziert, etwa für spezielle LKW-Spuren oder funkende Verkehrsschilder. Zu untersuchen wären weiters die Auswirkungen des zunehmend einfachen autonomen Individualverkehrs und neuer Nutzungsmodelle auf die Raum-und Verkehrsplanung. Die Untersuchung der genannten und anderer langfristigen Auswirkungen für Wirtschaft, Umwelt und Gesellschaft steht noch aus.

\section{Zitierte Literatur}

Krieger-Lamina, J., 2016, Vernetzte Automobile. Datensammeln beim Fahren - von Assistenzsystemen zu autonomen Fahrzeugen. Endbericht, No. 2016-02, 2016-08-31, Wien epub.oeaw.ac.at/ita/itaprojektberichte/2016-02.pdf.

Maurer, M., Gerdes, J. C., Lenz, B. and Winner, H. (Eds), 2015, Autonomes Fahren. Technische, rechtliche und gesellschaftliche Aspekte: Springer springer.com/de/book/9783662458532.

3 Siehe auch derstandard.at/2000102373920/Weniger-Unfaelle-durchautonomes-Fahren.

4 Siehe beispielsweise den Bericht der einschlägigen deutschen Ethikkommission vom Juni 2017, bmvi.de/SharedDocs/DE/Publikationen/DG/bericht-der-ethikkommission.html. 


\section{Selbstheilende Materialien}

Die meisten Materialien verschlechtern sich im Laufe der Zeit aufgrund von Ermüdung, Umweltbedingungen oder Schäden, die während der Nutzung auftreten. Risse und andere Arten von Schäden auf mikroskopischer Ebene verändern die thermischen, elektrischen und akustischen Eigenschaften von Materialien, Risse können dazu führen, dass das Material völlig unbrauchbar wird. Selbstheilende Materialien sind synthetisch hergestellte Substanzen, die die Fähigkeit haben, Schäden an sich selbst ohne externe Diagnose des Problems oder menschliches Eingreifen automatisch zu reparieren. Diese Materialien besitzen also "eingebaute" Reparaturmechanismen. Selbstheilende Materialien sind vielfach Polymere oder Elastomere, aber auch Metalle, Glas, Keramiken und Zement - letzterer könnte in Zukunft zu deutlich längeren Gebrauchszeiten für Häuser und Straßen führen. ${ }^{1}$ Die Heilungsmechanismen reichen von einer intrinsischen Reparatur des Materials bis hin zur Zugabe eines Reparaturmittels, welches durch einen äußeren Reiz (Licht, Temperaturänderung, usw.) aktiviert wird. In einem aktuellen wissenschaftlichen Artikel haben japanische Forscher eine neue Klasse selbstheilender Polymere entwickelt. Diese so genannten Copolymere weisen nach mechanischer Beanspruchung außerordentliche Selbstheilungsfähigkeiten, sowohl in trockenen als auch wässrigen und alkalischen Milieus auf. Die Heilung erfolgt dabei innerhalb weniger Minuten (Wang et al. 2019). Einige selbstheilende Materialien gelten als intelligente Strukturen, da sie sich an verschiedene Umgebungsbedingungen anpassen können. Selbstheilende Materialien können durch ihre besonderen Eigenschaften zu einer höheren Kosteneffizienz führen. Sobald ein Material Gebrauchsschäden aufweist, würde es sich selbst reparieren und müsste nicht aufwendig ausgetauscht werden. Die Lebensdauer von Teilen könnte erhöht werden, was vor allem im industriellen Einsatz von wesentlicher Bedeutung wäre.

Die Anwendungsfelder für solche neuartige Materialien sind sehr breit und zukunftsträchtig. ${ }^{2}$ Sie reichen von Materialien im Flugzeugbau über Brennstoffzellen bis zu Oberflächenbeschichtungen. International gibt es bereits erste Firmen, die selbstheilende Materialien kommerziell erzeugen, z. B. der französische Chemiekonzern Arkema (Gummi) oder die US-Firma Autonomic Materials (Korrosionsschutz). Auch in Österreich gibt es dazu einige F\&E-Aktivitäten, beispielsweise: recycelbare Kunststoffe, die das Polymer-Kompetenzzentrum Leoben entwickelt; ${ }^{3}$ selbstheilende Metalle, mit denen sich das Erich-Schmid-Institut für Materialwissenschaften der ÖAW

\footnotetext{
1 intechopen.com/online-first/self-healing-concrete-and-cementitious-materials.

2 Radical Innovation Breakthrough Inquirer (RIBRI): Foresight Tender EC: Fraunhofer ISI (lead), Institut Prospectiva, Finland Futures Research Centre, ribriconsultation.eu; siehe auch den ausführlichen Wikipedia-Artikel "Self-healing material", Stand: 5.5.2019, en.wikipedia.org/wiki/Self-healing_material.

3 uar.at/en/associated-companies/associated-companies-/polymer-competencecenter-leoben-gmbh/recyclable-and-self-healing-polymer-materials.
} 
und die Montan-Uni Leoben beschäftigen. ${ }^{4}$ Österreich ist über Joint Technology Initiative ECSEL-Austria auch an den europäischen Aktivitäten zur Entwicklung von elektronischen Komponenten und Systemen beteiligt. ${ }^{5}$

4 pure.unileoben.ac.at/portal/de/publications/thermallytriggered-dual-insituselfhealing-metallic-materials(28345444-215c-4692-acb6-3822480669dc).html.

5 ecsel-austria.net/home. 


\section{Industrie 4.0 und Bioökonomie}

Industrie 4.0 steht für die vierte industrielle Revolution durch intelligente, digital vernetzte Produktionssysteme (siehe Thema "Industrie 4.0"). Die Bioökonomie bezeichnet den Paradigmenwechsel von der erdölbasierten, fossilen zur biobasierten Produktion. Die Umbrüche der Digitalisierung mit Industrie 4.0 und der biobasierten Ökonomie (OECD 2009) sind jeweils Gegenstand von politischen Strategien. Innovationen mit hoher Reichweitezeichnen sich an der Schnittstelle von Bioökonomie und Industrie 4.0 ab. ${ }^{1}$

Die wechselseitigen Impulse lassen sich in verschiedenen Bereichen feststellen: Die Digitalisierung und die vernetzte, adaptive Produktion können die Effizienz der bestehenden biobasierten Produktionsprozesse steigern. Anwendungsfelder sind das Management von Rohstoffströmen², neue Logistiklösungen und maßgeschneiderte Lösungen für spezifische Anwendungen (z.B. Online-Energiemanagement-Plattformen in der Nutzung von Biomasse). Die Entwicklung neuer biobasierter Produkte wie etwa Verbundstoffe, Biopolymere, Bau- und Dämmstoffe, Biotreibstoffe, Chemikalien (vgl. Hatti-Kaul et al. 2007), Pharmazeutika und biobasierte Spezialprodukte sind auf digitale Technologien angewiesen, um eine gleichbleibende Qualität zu gewährleisten. Zugleich werden neue Geschäfts- und Betriebsmodelle für ermöglicht, die über die Digitalisierung in industrielle Prozesse eingebunden werden können. In der Automobilindustrie, die im Bereich Industrie 4.0 führend ist, sind unter anderem naturfaserverstärkte Karosserieteile und biokunststoffbasierte Innenverkleidungen möglich. Digitale Plattformen, digitale Analytik und digitale Verfahrens- und Anlagentechnik, die in der biomedizinischen-und Pharmaforschung heute zentral sind, können für biobasierte Industriematerialien umfassend zum Einsatz kommen. Sie ermöglichen Innovationen in der Systembiologie, in der synthetischen Biologie oder auch in der Mikrobiomforschung und ihrer Anwendung. Beispiele sind biochemisch veränderte Tabakpflanzen, die als grüne Arzneifabriken Medikamente produzieren ${ }^{3}$. Zudem lässt sich über die Digitalisierung die Transparenz und Offenheit in der biobasierten Produktion erhöhen. Über das Internet können kleine, primär regional tätige Unternehmen globale Reichweite und Sichtbarkeit und Einbindung erreichen (vgl. Pyka\&Buchmann 2017).

In Österreich zeigen sich erste Initiativen, die diese Schnittstelle adressieren, v.a. im industriellen Bereich nachwachsender Rohstoffe (Holzverbundwerkstoffe) und einzelnen Spezialsegmenten (Mikroalgen, Wirk-stoff-

1 Thematisiert wird es am Rande der Bioökonomie-FTI-Strategie für Österreich. Im Rahmen der FFG-Ausschreibung zur Produktion der Zukunft gab es bereits einen Subschwerpunkt zur Prozessentwicklung in der Biobasierten Industrie.

2 Z.B. durch den Einsatz von Ortungssystemen zum Rohstoff-Monitoring (siehe bspw. Future of CAP: Monitoring from outer space)

3 Bespiele sind belastbare Naturfaserverbundstoffe für die Automobilindustrie, Digitale Zwillinge in der Wirkstoffproduktion oder auch gedruckte Papiersensoren in der Holzindustrie. 
herstellung) (Ganglberger et al. 2017). Während Österreich im industriellen Bereich gut aufgestellt ist, fällt das Thema Bioökonomie 4.0 auf Policy-Ebene zwischen die Verantwortlichkeiten verschiedener Ministerien (z.B. bmvit, BMNT) und bedarf zukünftig sektorübergreifender Ansätze einer transformativen Innovationspolitik. Ob das hohe Innovationspotential genutzt werden kann, wird allerdings auch stark davon abhängen, in welcher Form, Intensität und Geschwindigkeit die Akteure auf dem Feld durch Förderung und Bewusstseinsbildung zusammengebracht werden.

\section{Zitierte Quellen}

Ganglberger, E., Sturm, T., Zahradnik, G. und Scherngell, T., 2017, Quantitative Indikatoren für die Biobasierte Industrie in Österreich, im Auftrag von: bmvit Bundesministerium für Verkehr, I. u. T., Wien.

Hatti-Kaul, R., Tornvall, U., Gustafsson, L. und Borjesson, P., 2007, Industrial biotechnology for the production of bio-based chemicals - a cradle-tograve perspective, Trends Biotechnol 25(3), 119-124; auch veröffentlicht in: Trends Biotechnol.

OECD, 2009, The Bioeconomy to 2030: designing a policy agenda Paris: OECD.

Pyka, A. und Buchmann, T., 2017, Die Transformation zur wissensbasierten Bioökonomie, in: Burr, W. und Stephan, M. (Hg.): Technologie, Strategie und Organisation, Wiesbaden: Springer Fachmedien Wiesbaden, 333-361. 


\section{Smart Spaces}

Sogenannte „Intelligente Räume“ („Smart Spaces“) bilden eine physische und/oder digitale Umgebung, in der Menschen mit unterschiedlichsten Technologien interagieren (Cearley/Burke 2018). Sie reichen von Büros mit moderner IT-Infrastruktur und ausgestattet mit einer Reihe von Sensoren, die sowohl den Raum als auch das Verhalten der Benutzerlnnen überwachen und steuern, ${ }^{1}$ bis zu Arbeitsumgebungen, in denen sogenannte „Digitale Zwillinge“ („Digital Twins“) mit den BenutzerInnen interagieren.

Digitale Zwillinge sind digitale Modelle oder Repräsentationen (Abbilder in Software) von Menschen, Dingen und organisatorischen Abläufen mit allen relevanten Eigenschaften und Verknüpfungen. Sie werden zunehmend zur Überwachung, Analyse und Kontrolle realer Umgebungen eingesetzt. ${ }^{2}$ Diese Digitalen Zwillinge in Kombination mit Künstlicher Intelligenz $(\mathrm{KI})$ und Erfahrungen aus der Nutzung Virtueller und Augmentierter Realitäten (VR und AR) bilden die Grundlage für offene, verbundene und koordinierte, intelligente Räume. Ziel ist die Integration von Menschen und verschiedenen digitalen Einheiten in „intelligenten Ökosystemen“, die ihre Aktivitäten koordinieren und so zu mehr Automation und zu Effizienzsteigerungen beitragen sollen/können. Als Zielvorstellung eines Intelligenten Raums soll es digitale Zwillingsmodelle von Menschen, Prozessen und Dingen in einer Stadt geben (Cearley/Burke 2018).

Das Wesen digitaler Räume liegt in der Kombination und Integration verschiedenster Technologien. So werden Aspekte des Internet of Things (IoT), Augmented Reality und Edge Computing, also einer anderen Softwarearchitektur auf Systemebene, ${ }^{3}$ genauso eingesetzt wie KI-Anwendungen. In der größten Ausprägung entspricht ein Intelligenter Raum dem Konzept der umfassenden Smart City, die wiederum mehrere Fragen bezüglich der entstehenden Risiken aufwirft. ${ }^{4}$

Bei den erwarteten Steigerungen des Marktvolumens in diesem Bereich ${ }^{5}$ stellt sich allerdings die Frage, inwieweit in dieser Vision über menschliche Bedürfnisse hinweg gegangen und Grundrechte eingeschränkt werden sollen. Kann ein möglichst effizientes Funktionieren tatsächlich das oberste Ziel sein? Und wie können zukünftige Arbeits- und Lebenswelten so gestaltet werden, dass Menschen sowie soziale und kulturelle Eigenheiten und Errungenschaften nicht völlig dem Druck zu Systemeffizienz untergeordnet werden?

\footnotetext{
1 it-business.de/smart-spaces-vernetzung-statt-verwaltung-a-913901/.

2 In sog. Connected Cars aber auch in der Überwachung von Produktionsmaschinen und ganzen Fertigungsstraßen. Siehe auch Digital Energy Twin projekte.ffg.at/projekt/3308396.

3 Details siehe Cearley/Burke (2018) und auch Thema „KI-Lernen“.

4 repository.corp.at/545/1/CORP2019_93.pdf.

5 www.alliedmarketresearch.com/smart-space-market.
} 


\section{Zitierte Literatur}

Cearley, D. und Burke, B., 2018, Top 10 Strategic Technology Trends for 2019, Gartner, gartner.com/en/doc/3891569-top-10-strategictechnology-trends-for-2019. 


\section{Lebensmitteltracking}

Mit neuen Technologien der Sensorik und Überwachung kann im Lebensmittelbereich eine neue Stufe der Sicherheit für KonsumentInnen erreicht werden. Ebenso könnten Fortschritte in der Abfallvermeidung und damit Ressourcenschonung erzielt werden. Nahrungsmittelsicherheit ist global ein wichtiges Thema. Insbesondere die Zurückverfolgung verdorbener, giftiger Lebensmittel stellt sich dabei als schwierig und zeitraubend dar. Oft müssen ganze Chargen vernichtet werden, was zum ohnehin riesigen Berg an Lebensmittelabfall beiträgt. Mithilfe der Blockchain-Technologie könnte hier ein Durchbruch gelingen. Wenn alle Rohstoffe und verarbeiteten Lebensmittel vom Bauernhof bis zur Gabel des/r Konsumentln ("farm2fork"1) in einer Blockchain registriert sind, lässt sich die Rückverfolgbarkeit wesentlich schneller und vor allem punktgenau durchführen. Große US-amerikanische Unternehmen der IT-Industrie sind bereits dabei, derartige Plattformen aufzubauen (World Economic Forum 2019).

Neben den Schäden aus Lebensmittelvergiftungen ist vor allem die Frage des Lebensmittelabfalls ein großes Problem. Etwa 30\% der jährlichen Lebensmittelproduktion wird weggeworfen (Kundua et al. 2019). In Ländern des globalen Südens geht mehr im Bereich der Verarbeitung verloren, während bei uns mehr im Verkauf bzw. bei den KonsumentInnen verloren geht.

Ein wichtiger Schritt in Richtung Lebensmittelsicherheit ist diesbezüglich in der Sensortechnologie getan worden. Durch Biosensoren, die beispielsweise als Label in neuartige Verpackungen integriert werden, ist es möglich, unterschiedlichste Parameter der Lebensmittelgüte zu überwachen: von der Lagertemperatur während der Lieferkette ${ }^{2}$ über den Reifegrad von Früchten bis zur Frische von Fleisch (ibid., S. 163). Damit kann auch für KonsumentInnen angezeigt werden, wie lange ein Lebensmittel noch genießbar ist, und so dem frühzeitigen Wegwerfen Einhalt geboten werden. Diese neuen Biosensoren können zusätzlich noch mit RFID-Tags ausgestattet werden und über eine entsprechende IT-Infrastruktur bei Transport und Handel ihre Daten weiterleiten und so jederzeit aus der Ferne überwacht werden.

\section{Zitierte Literatur}

Kundua, M., Krishnana, P., Kotnalab, R. K. und Sumanab, G., 2019, Recent developments in biosensors to combat agricultural challenges and $T$ their future prospects, Trends in Food Science \& Technology 88(June 2019), 157-178 doi.org/10.1016/j.tifs.2019.03.024.

World Economic Forum, 2019, Top 10 Emerging Technologies 2019, WEF, weforum.org/reports/top-10-emerging-technologies-2019.

1 Siehe dazu die EU Policy: ec.europa.eu/food/farm2fork_en.

${ }^{2}$ Ein österreichisches Unternehmen bietet derartige Sensoren für medizinische Güter an (www.suessco.com/sensors/healthcare/). 


\section{Metalinsen}

Seitdem die ersten Mobiltelefone mit eingebauter Video- und Fotokamera Anfang der 2000er-Jahre auf den Markt gekommen sind, haben sich unser Alltag und unsere Kommunikation stark verändert. Heute sind wir jederzeit in der Lage, Fotos und Videos zu machen und diese auch mit "der Welt" zu teilen. Mobiltelefone haben mittlerweile nicht mehr nur eine Kamera verbaut, sondern teilweise bereits bis zu sechs - Tendenz steigend. Dieser Trend verdeutlicht, dass Kamerasysteme unseren Altag in immer größerem Ausmaß durchdringen (z.B. auch in U-Bahnen, an öffentlichen Orten, etc.). Und er könnte sich mit der Entwicklung von so genannten Metalinsen noch weiterhin verstärken.

Bei Metalinsen handelt es sich um Linsensysteme, die nicht auf dem Prinzip der klassischen konkaven und konvexen Glas- oder Kunststofflinsen, die wir aus unserem Alltag kennen, beruhen. Eine Metalinse besteht aus einem dünnen, lichtdurchlässigem Trägermaterial, auf das Nanostrukturen in Form von Rillen und Säulen mittels lithographischen Verfahren gefräst werden. Es handelt sich um eine sogenannte Fresnel-Linse (Anwendung z.B. in Leuchttürmen), jedoch im nanoskaligen Bereich. ${ }^{1}$ Der Vorteil von Metalinsen liegt vor allem darin, dass eine Miniaturisierung von optischen Systemen möglich wird, die durch konventionelle Linsensysteme nicht erreichbar wäre. Damit ergeben sich viele neue Anwendungsmöglichkeiten. ${ }^{2}$ Die Kosten für optische Systeme (Sensoren, Endoskope, Lichtwellenleiter etc.) könnten durch Metalinsen ebenfalls reduziert werden (WEF 2019).

Ein Forschungsteam der Harvard Universität konnte eine Metalinse fertigen, die in der Lage ist, zu fokussieren und zu zoomen (She et al. 2018) eine wichtige Errungenschaft in Richtung Konsummarkt. Sobald Metalinsen den Konsumgütermarkt erreicht haben (Kameras, Virtual Reality Headsets etc.), könnte das einen großen Einfluss auf unseren Alltag nehmen. Eine starke Miniaturisierung von Kamerasystemen könnte eine gleichsam „unsichtbare“ Videoüberwachung ermöglichen, die vor allem in der Kombination mit Anwendungen im Bereich des Internets der Dinge inre Wirkung entfalten würde. Aus Sicht der Technikfolgenabschätzung gilt es hier rechtzeitig auf die potentiellen Folgen aufmerksam zu machen, zugleich aber die mit diesen technischen Errungenschaften verbundenen Chancen für die österreichische Wirtschaft zu berücksichtigen und zu erheben.

\footnotetext{
1 spektrum.de/news/wie-duenn-kann-eine-linse-sein/1630558.

2 Passend dazu siehe auch: Futuristische Sehhilfen.
} 


\section{Zitierte Literatur}

She, A., Zhang, S., Shian, S., Clarke, D. R. und Capasso, F., 2018, Adaptive metalenses with simultaneous electrical control of focal length, astigmatism, and shift, Science Advances 4(2), eaap9957 advances.sciencemag.org/content/advances/4/2/eaap9957.full.pdf.

WEF, 2019, Top 10 Emerging Technologies 2019: World Economic Forum www3.weforum.org/docs/WEF_Top_10_Emerging_Technologies_201 9_Report.pdf 


\section{Affective Computing - Emotionale Künstliche Intelligenz}

Digitale Systeme und Geräte, die menschliche Gefühle erkennen, interpretieren, verarbeiten und auf sie reagieren können, werden als Affective computing bezeichnet. Zukünftig sollen Stimmungen von Autofahrerlnnen anhand von Mimik, Gestik und Lautstärke erkannt und beurteilt werden. Ein Auto der Zukunft könnte bei extremer Wut oder Müdigkeit auch intervenieren. Intelligente Online-Lernsysteme könnten die Identifikation von Gefühlen wie Überforderung oder Langeweile nutzen, um eine personalisierte Lernerfahrung zu bieten und psychische Probleme könnten zunehmend über Apps frühzeitig identifiziert werden (vgl. el Kaliouby 2017). ${ }^{1}$ Digitale Systeme erkennen zunehmend die Gefühlslage derer, die sie nutzen und können entsprechend reagieren. Mittels digitaler Technik lassen sich menschliche Stimmungen und Gefühle immer besser bestimmen und zugleich werden immer mehr Interventionen entwickelt, auf die Stimmungen von Individuen zu reagieren bzw. diese zu nutzen und zu beeinflussen. ${ }^{2}$ Mit den persönlichen digitalen Assistenten verbreitet sich die Spracheingabe als Interaktion mit digitalen Geräten und aus den Spracheingaben der NutzerInnen lassen sich Gefühle nach Parametern wie Wortwahl, Tonhöhe und Pausen identifizieren. Optische Sensoren (Kameras) nutzen die Mimik, um Emotionen zu erkennen und mit den Archiven von Millionen von Gesichtern sind die führenden Unternehmen der Werbeindustrie in der Lage, z.B. umfangreiche Dienste auf Grundlage der emotionalen Wirkung von Werbeeinblendungen zu bieten.

Mit Technologien des Affective Computing erscheint NutzerInnen die Interaktion mit Maschinen komfortabler und natürlicher, das Vertrauen und die Nutzungsintensität nehmen zu, wodurch weitere Daten für die lernenden Programme geliefert werden. NutzerInnen reagieren auf die Programme, sie kaufen im Kontext der Interaktion und sie steuern zusätzlich weitere vernetzte Geräte. Somit wird der Zusammenhang von Gefühlen und Verhalten identifizierbar und kann ebenfalls für Marketing und Verhaltenskontrolle genutzt werden.

Die Nutzung von Technologien mit emotionaler Intelligenz wirft sowohl bei bewusster Nutzung im Arbeitsleben, im Verkehr, in Lernumgebungen und im Bereich psychischer Erkrankungen neue ethische, aber auch versicherungsrechtliche Fragen und Fragen des Datenschutzes auf. Noch dringender stellen sich die Fragen, wenn Emotionen ohne Zustimmung, d.h. unbemerkt, bei der Online-Nutzung erhoben und analysiert werden. Nachhaltige Innovationspfade zur Nutzung des hohen Potentials von An-

1 Die Anwendungen des Affective Computings kommen vielfach aus der medizinischen Forschung, so auch bei dem wichtigsten Unternehmen Affectiva: siehe el Kaliouby, et al. (2006).

2 Die Forschungsrichtung des Affective computing ist seit längerem etabliert, siehe z.B. Picard, et al. (2001), aber die Dynamik ist sehr stark durch die Anwendungen im Bereich persönlicher digitaler Assistenten bestimmt. 
wendungen des Affective Computing bedürfen eines Rahmens, der Vertrauen in die Technologien gewährleistet.

\section{Zitierte Quellen}

el Kaliouby, R., 2017, We Need Computers with Empathy, Technology Review 120(6), 8-9.

el Kaliouby, R., Picard, R. und Baron-Cohen, S., 2006, Affective computing and autism, in: Bainbridge, W. S. und Roco, M. C. (Hg.): Progress in Convergence: Technologies for Human Wellbeing, Malden: WileyBlackwell, 228-248.

Picard, R. W., Vyzas, E. und Healey, J., 2001, Toward machine emotional intelligence: Analysis of affective physiological state, leee Transactions on Pattern Analysis and Machine Intelligence 23(10), 1175-1191. 


\section{Gamification von Wissenschaft, Arbeit und Politik?}

Die Verbreitung von Onlinespielen in Unterhaltung und Populärkultur macht den Erfolg des Spielkonzepts deutlich: Kompetitive Settings und spieltypische (Bewertungs-)Elemente, wie Punkte, Ranglisten, Highscores und Spielniveaus (Level), erhöhen die Motivation bei der Erfüllung komplexer oder als monoton empfundener Aufgaben (Robson et al. 2015). Gamification, auf Deutsch so viel wie „Spielifizierung“, bezeichnet die Anwendung von Spieldesignelementen in nicht-spielerischen Kontexten (Deterding et al. 2011). Während Computerspiele vordergründig für Unterhaltung sorgen, zielen Gamification und ernsthafte Spiele bzw. Lernspiele, sogenannte Serious Games, auf bestimmte Ergebnisse ab, die sich auf den Alltag der SpielerInnen auswirken, d.h. realweltlichen Einfluss haben (Lernen, Motivation, Engagement, Leistungssteigerung und Spaß, Vermeidung unerwünschter Verhaltensweisen). Gamification und Serious Games stehen in direktem Zusammenhang mit institutionellen Zielen (Bildung, Wissenschaft, Regierung, Industrie) (Bogost 2007) und unterscheiden sich damit grundlegend von rein spielerischer Interaktion.

Die extensive Anwendung von Spielelementen in nicht-spielerischen Kontexten ist in unterschiedlichen Bereichen gegenwärtige Praxis (Bildung, Informationswissenschaften, Mensch-Maschine-Interaktion, Gesundheit). In der Krebsforschung wird Gamification eingesetzt, um die Erkennung von problematischen DNA-Fehlern zu verbessern. ${ }^{1}$ In Form eines Computerspiels wird die menschliche Sehkraft und Analysekapazität genutzt, um wiederkehrende und auffällige Muster in großen genetischen Datenmengen zu entdecken. Dazu begeben sich SpielerInnen mit einem Raumschiff auf die Suche nach dem wertvollen Element Alpha. Im Zuge ihrer Mission müssen sie eine Route zu den gesichteten Alpha-Vorkommen planen und markieren dabei auf einer Weltraumkarte auffällige Punkte, die gleichzeitig auffällige Muster in den Gendaten indizieren. Ein weiteres Online-Spiel aus der Forschung ist Foldlt ${ }^{2}$ - hier können SpielerInnen Proteine falten und dabei spielerisch neue Strukturen entwickeln, mit dem übergeordneten Ziel Proteinstrukturen vorherzusagen und neue Proteine zu designen.

Der Trend der Gamification hat auch in der Arbeitswelt Einzug erhalten. Hier zielt das kompetitive Spielprinzip auf die Motivationssteigerung bei Angestellten, auf die Erhöhung der kooperativen Zusammenarbeit und auf individuelle Anerkennung von Mitarbeiterlnnen. ${ }^{3}$ Unternehmen, wie PwC ${ }^{4}$ und Marriott International (Freer 2012) setzen unter dem Schlagwort „Recruitainment" in der Personalsuche auf Gamification und verwenden ei-

\footnotetext{
1 scienceblog.cancerresearchuk.org/2014/02/04/download-our-revolutionarymobile-game-to-help-speed-up-cancer-research/.

2 fold.it/portal/.

3 forbes.com/sites/jeannemeister/2015/03/30/future-of-work-using-gamificationfor-human-resources/\#eeab22024b7b.

4 multipoly.hu.
} 
gens programmierte Jobsimulationen, um potenziellen Angestellten ihren zukünftigen Arbeitsplatz während des Bewerbungsprozesses näher zu bringen und um sogenannte „High Potentials“ auszuwählen.

Planspiele ermöglichen es, die Funktionsweisen komplexer Systeme spielerisch zu erforschen. Ein Teilbereich sind politische Planspiele. ${ }^{5}$ Dazu gehören Spiele zur Funktionsweise von UN-Sicherheitsratssitzungen und zur Formulierung von Resolutionen in Bezug auf Konfliktgebiete ebenso wie auch Spiele zu internationalen Handelsregeln, die die Problematik von Entscheidungsfindung vermitteln sollen. Teilnehmende können in der Rolle von Regierungsmitgliedern ihre Kenntnisse in Volkswirtschaft, nachhaltiger Entwicklung und Umweltfragen entwickeln oder WertpapierDepots mit einem fiktiven Startkapital verwalten. Planspiele können nicht nur Bewusstsein für politische Prozesse schaffen, sondern auch in der Politik für die Erkundung von Möglichkeitsräumen genutzt werden. ${ }^{6}$

Gerade im Fall von komplexen politischen und sozialen Ereignissen, wie z.B. einer Naturkatastrophe oder einer Terrorattacke, können unterschiedliche Annahmen, Meinungen und Werte dazu beitragen, dass notwendige ethische und moralische Entscheidungen aufgeschoben werden und Maßnahmen, die der vorherrschenden ideologischen Haltung zuwiderlaufen würden, nicht umgesetzt werden. Persuasive Games - auf Deutsch „Überzeugungsspiele“ - sind eine spezifische Form der Serious Games, die darauf abzielen unterschiedlichen Weltsichten und implizite Annahmen explizit zu machen. Sie könnten damit zu alternativen Denkweisen und höherer Entscheidungskompetenz im Ernstfall führen. Wie Wirtschaft und Politik langfristig vom Gamification-Trend profitieren können und ob es dazu Standards geben sollte, ist noch offen.

\section{Zitierte Quellen}

Bogost, I., 2007, Persuasive games the expressive power of videogames, Cambridge, MA: MIT Press.

Deterding, S., Khaled, R., Nacke, L. und Dixon, D., 2011, Gamification: Toward a Definition, CHI 2011 Gamification Workshop Proceedings.

Freer, T., 2012, Social media gaming - a recipe for employer brand success, Strategic HR Review 11(1), 13-17.

Robson, K., Plangger, K., Kietzmann, J. H., McCarthy, I. und Pitt, L., 2015, Is it all a game? Understanding the principles of gamification, Business Horizons 58(4), 411-420.

5 Siehe z.B. die Datenbank der Bundeszentrale für politische Bildung in Deutschland: bpb.de/lernen/formate/planspiele/65585/planspiel-datenbank.

6 iiasa.ac.at/web/home/research/researchPrograms/EcosystemsServicesand Management/event/170614-WorldsFutureGame.html;

https://www.oecd-ilibrary.org/sites/9789264301061-5en/index.html?itemld=/content/component/9789264301061-5-en. 


\section{Intelligente und funktionelle Oberflächen für industrielle Anwendungen der Zukunft}

Elektronische Informations-, Bedien- und Messelemente werden durch die zunehmende Digitalisierung immer kleiner und vielfältiger einsetzbar. Eine zukünftige Anwendungsmöglichkeit bietet die Integration dieser elektronischen Teile in Oberflächen von Objekten oder auch in Folienumhüllungen. Während smarten Oberflächen im Gesundheitswesen unter dem Schlagwort „Printed Healthcare Diagnostics“ hohes Zukunftspotenzial zugeschrieben wird (siehe Elektronische Haut), gewinnen gegenwärtig vor allem industrielle Anwendungsfelder an Relevanz. Ein Beispiel dafür sind beheizte Kunststoffoberflächen, die z.B. im E-Automobilbereich energiesparend eingesetzt werden könnten. ${ }^{1}$ Denkt man das Potenzial dieser technologischen Innovation weiter, ist auch eine elektrisch beheizbare Wandfarbe möglich ${ }^{2}$, die effiziente Raumwärme garantiert. Umgemünzt auf Leuchteigenschaften könnten durch den Einsatz von organischen Leuchtdioden (OLEDs) leuchtende Tapeten statt Lampen oder überdimensionale Touchscreens unsere Wohnräume zukünftig verändern. ${ }^{3}$ Eine weitere, zukunftsträchtige Anwendung sind intelligente Fenster, die sich durch steuerbare Lichtdurchlässigkeitseigenschaften verdunkeln lassen und nicht nur vor Licht-, sondern auch vor Wärmeeinstrahlung schützen können. ${ }^{4}$ Materialforschung hat in Österreich lange Tradition und gerade deshalb gilt es bestehende Wissenspotenziale hin Hinblick auf Zukunftstechnologien auszuschöpfen und auch für den Industriesektor nutzbar zu machen. Die Suche nach neuen Kombinationen und alternativen Anwendungsmöglichkeiten für smarte Oberflächen steht hier im Fokus (Bernhard et al. 2019). Eine zentrale Herausforderung bei der Entwicklung intelligenter Oberflächen ist der multidisziplinäre Charakter: die langfristig stärkere Zusammenarbeit von Forschungseinrichtungen, Unternehmen bzw. Zulieferbetrieben, Anwenderlnnen und potenziellen NutzerInnen erscheint hier wünschenswert. Inwiefern hier innovative öffentliche Beschaffung Impulse setzen kann, könnte im Rahmen einer Foresight-Studie ausgelotet werden.

\section{Zitierte Literatur}

Bernhard, K., Leitner, P. und Peyrl, R., 2019, Smarte Oberflächen: Auf das Äussere kommt es an, Oberösterreichische Zukunftsakademie.

\footnotetext{
1 kunststoff-cluster.at/news-presse/detail/news/neue-technologie-fuer-beheiztepremium-oberflaechen/.

2 carbo-e-therm.de/e-paint/.

3 forschung-und-wissen.de/nachrichten/technik/oleds-machen-die-tapete-zumheimkino-13372274.

4 neuffer.at/elektrochromes-glas.php.
} 


\section{Frugale Innovation für heiße Sommer}

Der Klimawandel und damit einhergehende Extremwetterlagen, v.a. heiße Sommermonate, erhöhen bereits jetzt den Bedarf an Techniken zur Kühlung von Lebensräumen. Der Einbau von Klimageräten in privaten Wohnraum kann zu Konflikten führen, denkt man z.B. an die Lärmbelästigung (v.a. in geräuschsensiblen Innenhöfen) und kann in der Anschaffung und im Betrieb erhebliche Kosten und hohen Energieverbrauch verursachen. Der Trend zur Digitalisierung verstärkt diese Dynamik v.a. in der Industrie immens. So müssen z.B. Server nicht nur ständig gekühlt werden, sondern sie erfordern auch redundant angelegte Kühlsysteme, die bei Ausfall das Überhitzen der Server trotzdem verhindern können. Bezogen auf große gesellschaftliche Bedarfe (im sozialen Wohnbau oder auch in der digitalen Industrie) ist die Nutzung von energieintensiven Technologien keine nachhaltige und langfristige Lösung. Konzepte wie solare $\mathrm{CO}_{2-}$ neutrale Kühlung oder Fernkälte sind durch den Einsatz hochtechnologischer und ressourcenintensiver Anlagenkomponenten nicht nur im Kostenfaktor exklusiv, sondern bedeuten trotz effizienter Energienutzung zusätzlichen Energiebedarf bzw. -verbrauch (siehe dazu auch Kältetechnologien). Ein ganz anderer Ansatz, der den verengten Blick auf herkömmliche Kältetechnologien zu erweitern vermag und neue Möglichkeiten verspricht, ist frugale ${ }^{1}$ Innovation. Frugale Innovation bricht mit dem weit verbreiteten Paradigma des "immer mehr - immer besser" und baut auf technologische abgespeckte (low-tech), erschwingliche Produkte und Lösungen, die aber dennoch ihre Funktion effektiv erfüllen. Der Fokus liegt auf einfachen, robusten und anwendungsorientierten Konzepten, die auch für preissensible Konsumsegmente am Fuße der sozioökonomischen Pyramide leistbar sind. Soziale Innovation ist ein wesentlicher Bestandteil von frugaler Innovation: hier geht es um Lernen von anderen kulturellen Kontexten, die für eine gegebene Problemstellung relevante Hinweise für manchmal überraschende soziale oder organisatorischen Lösungsansätze liefern. Im Fall von Kältetechnologien wäre es z.B. sinnvoll, soziale Praktiken in Regionen zu untersuchen, die Hitzewellen seit langem gewohnt sind und adäquate Weisen gefunden haben, damit umzugehen. Neben sozialer Innovation umfassen frugale Innovationsansätze auch Design- und architektonische Innovationen. Neben der bekannten Fassadenbegrünung zur Gebäudekühlung ist der persische Badgir ${ }^{2}$ - auf Deutsch Windfänger - ein Beispiel für eine jahrtausendealte Klimaanlage, die als Vorbild für natürliche Lüftungssysteme bis heute Gültigkeit besitzt. In der ökologischen Architektur wird Schwerkraftlüftung, die ohne zusätzlichen Energiebedarf auskommt, zunehmend auch in öffentlichen Gebäuden eingesetzt (z.B. in Schulen und Sporthallen). Bleibt man in näheren kulturellen Gefilden wären Eisspeichersysteme historische Vorbilder (ursprünglich als Eislöcher oder Eiskeller), die u.U. weiterentwickelt werden

\footnotetext{
1 Frugal bedeutet so viel wie schlicht, sparsam, nutzbar, aber auch fruchtig.

2 fzarchiv.sachon.de/Zeitschriftenarchiv/Maler-und_Lackierermeister/2017/ 10_17/ML_10-17_36-40_Von_Wueste_und_Windfaengen.pdf\#all_thumb.
} 
könnten. In Anbetracht einer blühenden Maker-Szene in Österreich, die in FabLabs und Makerspaces kostengünstig innovative Lösungen entwickelt und erprobt, wären verstärkte Anreize für frugale Innovationen im Kühltechnologiebereich (z.B. durch öffentliche und breit zugängliche Wettbewerbe) überlegenswert. 


\section{Offene Mobilitätsplattformen zur Unterstützung der Verkehrswende?}

\section{Zusammenfassung}

Was haben Laborleder, plastikfressende Bakterien und Superholz gemeinsam? Diese neuen Entwicklungen basieren auf Prinzipien, die der Natur entstammen bzw. die von biologischen Materialien, Prozessen und Funktionsweisen inspiriert sind (siehe Thema „Biomimikry“). Die Bandbreite dieser sogenannten „bioinspirierten“ Materialien reicht vom Einsatz natürlicher Komponenten, wie z.B. schnellwachsenden Holzsorten, die in einem neuartigen, chemischen Verfahren und durch Ausnutzung von Nanostrukturen zu härterem und stabilerem Superholz umgewandelt werden, bis hin zu genetisch veränderten Bakterien, die Kollagen als Ausgangsstoff für die Herstellung von Laborleder produzieren. Der mögliche Beitrag von bioinspirierten Materialien zur Transformation einer erdölbasierten Ökonomie hin zu einer nachhaltigen Bioökonomie ist groß. Als Querschnittsmaterie bietet die Materialforschung Anknüpfungspunkte in den unterschiedlichsten Bereichen, angefangen von medizinischen Biomaterialien, über organische Verpackungsmaterialien bis hin zu synthetischen Nahrungsquellen. Die Forschung an bioinspirierten Materialien für die weiße Biotechnologie ermöglicht es, im großen Stil herkömmliche Stoffe durch erneuerbare Ressourcen zu ersetzen und damit industrielle Prozesse kostengünstiger und ökologischer zu gestalten. Der Einsatz bioinspirierter Materialien könnte zukünftig einen wesentlichen Beitrag zur Erreichung der UN-Nachhaltigkeitsziele ${ }^{1}$ zum Klimaschutz und zu nachhaltigen Konsum- und Produktionsbedingungen leisten.

\section{Überblick zum Thema}

Die Anwendung von bioinspirierten Materialien hat v.a. in der Medizin eine lange Tradition. Dabei geht es um die gezielte Entwicklung und Modifikation von Materialien in Hinblick auf ihre funktionellen Eigenschaften. Dieser Paradigmenwechsel hin zu „Designed Biomaterials“ bzw. „Smart Materials“ lässt neben den klassischen Ansätzen aus Medizin und Materialwissenschaft vor allem neue Ideen aus der Biologie in Erscheinung treten (Ratner et al. 2013).

Biobasierte Materialien umfassen das Design und die Modellierung von neuen Werkstoffen nach dem Vorbild der Natur. Die stoffliche Ausgangsbasis kann natürlich (z.B. Biopolymere, wie Proteine, Zellulose, DNA) als auch synthetisch (Kunststoffe, Verbundwerkstoffe) sein und organische wie anorganische Komponenten enthalten. Methodische Treiber für die Entwicklung von bioinspirierten Materialien sind z.B. Genom-Editierung oder 3D-Druck. Die Genom-Editierung ermöglicht die zielgerichtete Ver-

„Designed Biomaterials" „Smart Materials"

Vorbild Natur

\footnotetext{
1 un.org/sustainabledevelopment/sustainable-development-goals/.
} 
Superholz Superholz bezeichnet einen neuen Werkstoff, der in einem zweistufigen Verfahren aus weichen Holzsorten hergestellt wird. In einem ersten Schritt wird das Holz durch chemische Behandlung aufgespalten, das Lignin und die Hemicellulose entfernt; dann wird das behandelte Holz heiß gepresst. Dadurch brechen die natürlichen Zellwände zusammen und es entstehen spezielle Nanofasern aus der Zellulose (Song et al. 2018). Das entstehende Material ähnelt Holz, ist aber um ein vielfaches härter und zäher. Aufgrund des Gewichtsvorteils gegenüber Stahl könnte Superholz in der Flugzeug- und Automobilerzeugung oder generell als nachhaltiger Baustoff eingesetzt werden².

Leder ist ein Kuppelprodukt, das zu 99\% von Tieren stammt, die für die Woll-, Milch und/oder Fleischerzeugung gehalten wurden (COTANCE/IndustriALL 2012). Obwohl die Ledererzeugung ein europäisches Traditionshandwerk darstellt und der natürliche Rohstoff kostengünstig verfügbar ist, wird Leder zu einem Großteil aus Billiglohnländern importiert. Der Grund dafür liegt in der hohen Umweltbelastung des Herstellungsprozesses, der in Europa zu strengen Auflagen und einer nachteiligen Position im globalen Wettbewerb geführt hat.

Eine Alternative zu herkömmlichen Leder und dem traditionellen Gerbungsprozess stellt im Labor gezüchtetes Leder dar. Hergestellt wird Laborleder durch genveränderte Hefezellen, die flüssiges Kollagen produzieren, welches anschließend in Form gebracht wird und in einem vereinfachten und umweltfreundlichen Gerbvorgang fertiggestellt wird. Laborleder könnte einerseits eine konstante Qualität garantieren und wäre andererseits nicht mit der hohen Schadstoffbelastung der traditionellen Lederverarbeitung verbunden. Durch kontrollierte Verfahren ist eine zeitgerechte Verfügbarkeit gegeben; zurzeit dauert es rund zwei Wochen, ein kuhhautgroßes Stück Laborleder wachsen zu lassen. Innovationen in der Verarbeitungsindustrie könnten sich dadurch ergeben, dass sich vielfälti-

2 wired.de/collection/science/super-dicht-super-stark-wissenschaftler-stellensuper-holz-her. 
ge Eigenschaften des Werkstoffs, z.B. die mechanischen Eigenschaften wie die Steifigkeit, durch die Nährstoffe, die zur Produktion eingesetzt werden, bestimmen lassen (Haneef et al. 2017).

Myzelien (Geflechte aus Pilzfäden) sind Kunststoffen aus fossilen Polymeren sehr ähnlich und gleichen äußerlich expandiertem Polystyrol (EPS). Sie bestehen aus Biopolymeren, wie z.B. Zellulose, Chitin und Proteinen. Derzeit werden Stoffe aus Myzelien vor allem in der Kunst (Moonboots aus Myzelien im MoMA New York) und als Verpackung eingesetzt ${ }^{3}$. Bisher ist aus Myzelien erzeugtes Material ein teures Nischenprodukt. Zukünftig wird dem bioinspirierten Material hohes Potenzial, vor allem in der Anwendung als Baustoff, zugeschrieben.

Plastik ist ein vielseitig einsetzbarer Werkstoff, der die Industrieproduktion des 20. Jahrhunderts wesentlich geprägt hat. Die Resistenz des Materials gegenüber natürlichen Abbauvorgängen macht die Entsorgung von Plastik zu einem globalen Umweltproblem, vor allem für marine Ökosysteme. Vor einigen Jahren entdeckten japanische Forscher Bakterien, die Kunststoff allmählich zersetzen können und entwickelten diese weiter. 2018 haben britische und US-WissenschafterInnen eine optimierte Enzymvariante gefunden, die PET um ein Vielfaches schneller zersetzt. Nun versuchen sie das Enzym für das bioinspirierte Recycling von Plastik nutzbar zu machen (Austin et al. 2018).

\section{Relevanz des Themas für das Parlament und für Österreich}

In den Medien sind neue bioinspirierte Materialien mit weitreichenden Zukunftsversprechungen verknüpft. Die Erwartungen an diese Materialien umfassen die vollständige Substitution erdölbasierter Materialien, bis hin zur Reinigung der Meere von Plastikteilen. Wenig Aufmerksamkeit kommt den unter Umständen weitreichenden Konsequenzen einer Verbreitung von bioinspirierten Materialien zu, die eng mit dem Diskurs über Gen- und Nanotechnologie verbunden sind. Was sind beispielsweise die Langzeitfolgen, die sich aus der Anwendung von genetisch veränderten Stoffen ergeben (gerade im Bereich Ernährung und Gesundheit)?

Je nach Herstellungsverfahren und Anwendungskontext stehen unterschiedliche ethische Fragen zur Debatte. Wenn es um Biomaterialien zum Einsatz in der Medizin geht, ist die Frage der Tierversuche, die zur Erprobung der Verträglichkeit neuer Stoffe notwendig sind, zentral. Ebenso ist die Frage der Patentierung und Kommerzialisierung von lebensrettenden bzw. -verlängernden Materialien in Hinblick auf inre Verfügbarkeit relevant. Bioinspirierte Materialien, wie z.B. das Laborleder, können dagegen zu Fertigungsprozessen beitragen, die auf Tierversuche verzichten können, ökologisch verträglich sind und langfristig eine Abkehr von der erdölbasierten Produktion hin zu einer Bioökonomie bedeuten.
Myzeltextilien

plastikabbauende

Enzyme

öffentlicher Diskurs

ethische Fragen

3 ecovativedesign.com. 
bestehende

Wissensbasis/

ExpertInnenpool
In Österreich hat die Materialforschung Tradition und eine starke Wissens- und Forschungsbasis. Während im Wood-K-Plus-Zentrum an der Entwicklung von Holz-Verbundwerkstoffen geforscht wird, befassen sich ForscherInnen der Montanuniversität mit Metallurgie und der Weiterentwicklung von metallischen Rohstoffen. Die Universität für Bodenkultur hat einen Forschungsschwerpunkt im Bereich der biotechnologischen Materialien, die Johannes Kepler Universität Linz beforscht bioinspirierte Materialien und Sensoren, sowie biokompatible Oberflächen und die Universität Innsbruck untersucht die Gesamtheit an Materialien unter dem Titel „Advanced Materials“. Das Ausnutzen und Vernetzen dieser bestehenden Wissens- und Forschungsbasis könnte Österreich zu einer Vorreiterrolle im Bereich der bioinspirierten Materialen führen.

\section{Vorschlag weiteres Vorgehen}

Für das Thema „Bioinspirierte Materialien“ wäre eine Zusammenschau der unterschiedlichen Werkstoffbereiche (Holz, Metall, Kunststoff etc.), die bioinspirierte Materialien beforschen, in Form einer Kurzstudie interessant. Anhand einer Intensivierung der Interdisziplinarität und eines Austauschs über die wissenschaftlichen Felder (angefangen von Medizin bis hin zur Verbundwerkstofftechnik) hinweg, könnten zusätzliche Potentiale von bioinspirierten Materialien ausgelotet werden. Im Rahmen einer Langstudie könnte eine Stakeholderanalyse durchgeführt sowie ein partizipativer Prozess zur Identifikation von Zukunftspotentialen für bioinspirierte Materialien in Österreich neue Impulse für die Forschung setzen und das Beschreiten neuer Innovationspfade in Hinblick auf die Anwendung von bioinspirierten Materialien motivieren.

\section{Zentrale weiterführende Quellen}

Austin, H. P., Allen, M. D., Donohoe, B. S., et al., 2018, Characterization and engineering of a plastic-degrading aromatic polyesterase, Proceedings of the National Academy of Sciences.

Bernhard, K., Leitner, P. und Peyrl, R., 2019, Smarte Oberflächen: Auf das Äussere kommt es an, Oberösterreichische Zukunftsakademie.

Cao, Y., Feng, Y., Ryser, M. D., et al., 2017, Programmable assembly of pressure sensors using pattern-forming bacteria, Nature Biotechnology 35, 1087, dx.doi.org/10.1038/nbt.3978.

COTANCE und IndustriALL, 2012, Sozial und Umweltbericht. Die Europäische Lederindustrie, Brüssel, cotance.com/socialreporting/SER/ESERGerman.pdf.

Haneef, M., Ceseracciu, L., Canale, C., Bayer, I. S., Heredia-Guerrero, J. A. und Athanassiou, A., 2017, Advanced Materials From Fungal Mycelium: Fabrication and Tuning of Physical Properties, Scientific Reports 7, 41292, dx.doi.org/10.1038/srep41292.

Ratner, B. D., Hoffman, A. S., Schoen, F. J. et al., 2013, Biomaterials Science. An Introduction to Materials in Medicine, $3^{\text {rd }}$ ed.: Academic Press, sciencedirect.com/science/article/pii/B9780080877808001480.

Schaffner, M., Faber, J. A., Pianegonda, L., et al., 2018, 3D printing of robotic soft actuators with programmable bioinspired architectures, Nature communications 9(1), 878, doi.org/10.1038/s41467-018-03216-w. 
Song, J., Chen, C., Zhu, S., Zhu, M., et al., 2018, Processing bulk natural wood into a high-performance structural material, Nature 554, 224, dx.doi.org/10.1038/nature25476. 


\section{Zellfabriken der Zukunft}

Eine Zukunftsvision in der Herstellung neuartiger Materialien besteht darin, lebende Zellen und ihre molekularen Komponenten umfassend als Zellfabriken im industriellen Maßstab zu nutzen. Um Mikroorganismen für die Produktion spezieller Stoffe einzusetzen, verwendet die Biotechnologie das Verfahren des Metabolic engineering (siehe Thema „Künstliches Leben“). Bei diesem Design von maßgeschneiderten Stoffwechsel- und Synthesewegen in einer Zelle können genetische Steuerelemente und Biosynthese-Gene aus Pflanzen, Tieren oder Mikroorganismen miteinander kombiniert werden, um Mikroben in effiziente Zellfabriken zu verwandeln - und sie etwa für die Herstellung von Medikamenten zu nutzen.

Zu den Musterbeispielen gehört die Produktion des Malariawirkstoffs Artemisinin, der traditionell aus der einjährigen Beifuß-Pflanze Artemisia annua extrahiert wird. Die Vorstufe des Wirkstoffs, Artemisininsäure, lässt sich auch von einer gentechnisch veränderten Hefe, einer „Designermikrobe", produzieren, aus der sich das gewünschte Malaria-Medikament herstellen lässt. ${ }^{1}$ Mittlerweile sind auch synthetische Hefezellen-Fabriken im Labormaßstab möglich, die in Zukunft den potenziellen Krebswirkstoff Noscapin produzieren können ( $\mathrm{vgl}$. Li et al. 2018). Manche der vielversprechenden Zellfabriken stellen allerdings zugleich bisherige internationale Regulierungsätze in Frage: Hefepilze lassen sich so verändern, dass sie opium-ähnliche Stoffe produzieren können, womit in Zukunft hochwirksame Schmerzmittel aus einfachsten Materialien entwickelt werden könnten. ${ }^{2}$ Auch wenn die Herstellung nicht im "Garagenlabor" möglich sein soll, so stellen sich mit Zellfabriken wie in der Opiatherstellung neue Fragen hinsichtlich Open Science als auch im Umgang mit organisierter Kriminalität. $^{3}$

Neben den medizinischen Anwendungen sollen Bakterien als Zellfabriken zum Beispiel auch natürliche Kunststoffe (siehe Thema „Industrie 4.0 und Bioökonomie“) mit hoher Ausbeute und Reinheit fertigen („Bio-Nylon“4).

\footnotetext{
1 Aufgrund der hohen Produktion an landwirtschaftlich erzeugtem Artemisinin und dem entsprechenden Preisverfall wurde das synthetische Verfahren aus Hefe kein Markterfolg, hat aber die Innovationstätigkeit auf dem Gebiet stark forciert: Mittlerweile transferierten ForscherInnen den entsprechenden Stoffwechselweg aus dem einjährigen Beifuß in die großblätterige Tabakpflanze, die landwirtschaftlich großflächig angebaut werden kann und große Mengen an Artemisininsäure günstig produzieren kann Fuentes, et al. (2016).

2 Siehe Galanie, et al. (2015). Hochrelevant angesichts dessen ist, dass das International Narcotics Control Board INCB der Vereinten Nationen schätzt, dass mehr als drei Viertel der Weltbevölkerung keinen ausreichenden Zugang zu schmerzlindernden Medikamenten für HIVIAIDS, Krebs und sogar Geburten haben.

3 Open Science sieht die umfangreiche Bereitstellung der Ergebnisse von Forschung vor. Ergebnisse bereitzustellen, die die Möglichkeit bieten, aus einfachen Stoffen Vorstufen von Drogen herzustellen, wären in diesem Fall problematisch.

4 Vgl. ingenieur.de/technik/fachbereiche/biotechnik/bio-nylon-bakterien-kunststoffnachwachsenden-rohstoffen-produzieren:
} 
Die maßgeschneiderten Zellfabriken für die industrielle Bioproduktion von Chemikalien, Materialien und Treibstoffen werden allerdings auf gezielter gentechnischer Modifikation beruhen.

Die Risiken eines umfassenden Einsatzes von Zellfabriken sind noch nicht systematisch untersucht, aber die Möglichkeiten in der Medizin, in der bio-basierten Ökonomie und in der industriellen Anwendung sind sehr weitreichend.

\section{Zitierte Quellen}

Fuentes, P., Zhou, F., Erban, A., Karcher, D., Kopka, J. und Bock, R., 2016, A new synthetic biology approach allows transfer of an entire metabolic pathway from a medicinal plant to a biomass crop, Elife 5 .

Galanie, S., Thodey, K., Trenchard, I. J., Interrante, M. F. und Smolke, C. D., 2015, Synthetic Biology. Complete biosynthesis of opioids in yeast, Science 349(6252), 1095-1100.

Li, Y. R., Li, S. J., Thodey, K., et al., 2018, Complete biosynthesis of noscapine and halogenated alkaloids in yeast, Proceedings of the National Academy of Sciences of the United States of America 115(17), E3922-E3931. 



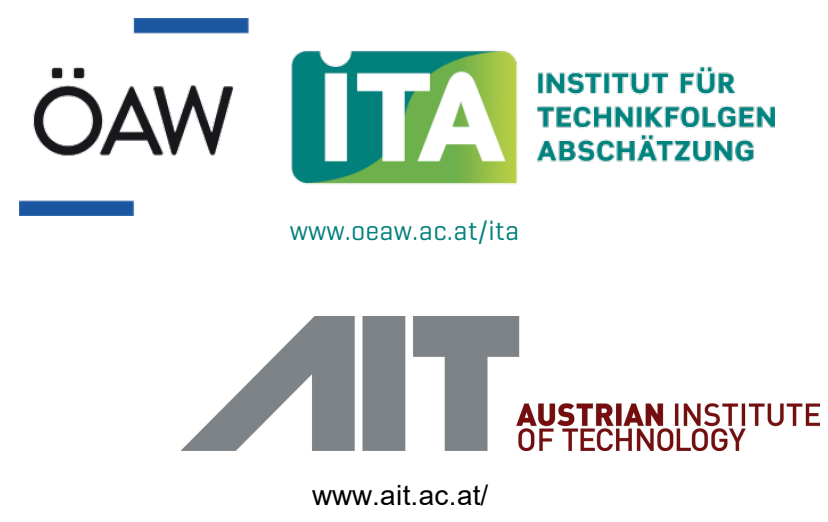

